\title{
A STUDY OF VEHICLE RESPONSE ASYMMETRIES DURING SEVERE DRIVING MANEUVERS
}

\author{
A Thesis \\ Presented in Partial Fulfillment of the Requirements for \\ the Degree Master of Science in the \\ Graduate School of The Ohio State University
}

By

Michael Daniel Dorohoff Jr., B.S.M.E

$* * * * *$

The Ohio State University

2003

Master's Examination Committee:

Approved by

Dr. Dennis Guenther, Adviser

Dr. Gary Heydinger

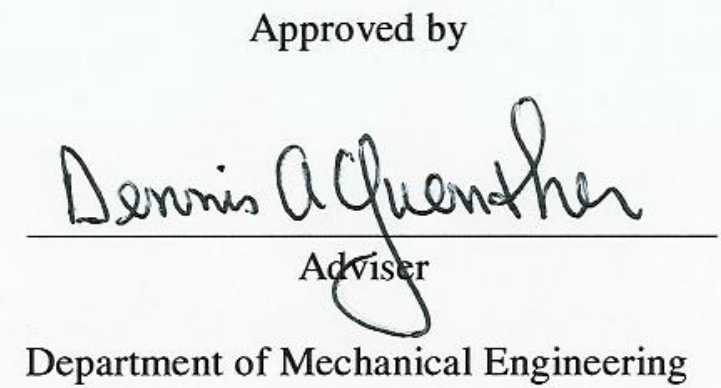




\begin{abstract}
The main objective of this research was to study vehicle response asymmetries during severe driving maneuvers. During Phase VI of the National Highway Traffic Safety Administration’s Light Vehicle Rollover Research Program, such response asymmetries occurred during field tests, and consequently, the data collected in Phase VI was used in this study. Of the twenty-six light vehicles tested in Phase VI, three vehicles exhibited significant response asymmetries with respect to left versus right steer maneuvers: the 1996 Acura SLX, 1995 Mitsubishi Montero, and 1993 Ford Aerostar.

An overview and an evaluation of several rollover stability indexes are provided to help explain the vehicle dynamics at the onset of rollover. No known models exist that incorporate vehicle asymmetries other than the lateral center of gravity (CG) offset. As such, this research effort investigates possible vehicle asymmetric characteristics and unintended inputs that may cause vehicle response asymmetries.

The cause of vehicle asymmetric response is quite complex and cannot be narrowed down to one item alone, e.g., CG offset. Careful consideration was given to possible unintended differences in the vehicle inputs that may adversely affect the vehicle response. These unintended vehicle inputs were determined to be either negligible or to have an insignificant impact on the vehicle asymmetric response, and therefore, the cause of vehicle asymmetric response is more likely within the vehicle itself. As such,
\end{abstract}


suspension and steering tests were conducted on the SLX and the Montero to identify any vehicle components/characteristics that may cause vehicle response asymmetries.

The suspension tests showed that both vehicles exhibited less stiffness in the nonlinear region of suspension compression on the side of the suspension that was opposite to the more severe steer direction in the J-turn maneuver. For example, the SLX experiences two-wheel lift (TWL) at a lower maneuver entrance speed in a left steer versus a right steer J-turn maneuver, and its right side suspension is softer than the left side. The same phenomenon applies to the Montero: it experiences TWL at a lower maneuver entrance speed in a right steer J-turn maneuver, and its left side suspension is softer.

To confirm the trend that the softer side suspension will lead to earlier TWL in the opposite steer direction, a model representative of the SLX was created and evaluated using CarSim ${ }^{\mathrm{TM}}$. Using the actual SLX suspension parameters, the model showed some differences between left and right steer response, but the effect of the lateral CG offset dominated the vehicle response.

The response of the shock absorbers was not determined during the suspension and steering tests, but a simulation study was done to evaluate the effect of the shock absorbers on vehicle response. Based on the shock absorber study, it is evident that a change in the damping force response can have a significant effect on the vehicle response. 
Dedicated to my parents and Jennifer 


\section{ACKNOWLEDGMENTS}

I wish to thank my adviser, Dr. Dennis Guenther, for his guidance, support, enthusiasm, and for teaching me the basics of vehicle dynamics.

I would also like to thank Dr. Gary Heydinger and Garrick Forkenbrock. Their assistance was invaluable to this research effort. I appreciate their willingness to discuss my research and share their knowledge.

In addition, I am grateful for all the other assistance that I received while working at the Vehicle Research and Test Center.

This research was supported by the Ohio State University through a University Fellowship and additional funding was provided by the National Highway Traffic Safety Administration. 
VITA

October 21, 1978....................................Born - Newark, Ohio

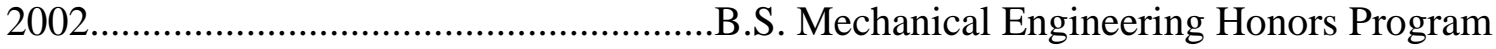
Ohio University, Athens, Ohio

2002 - present .........................................University Fellow The Ohio State University

Columbus, Ohio

\section{PUBLICATIONS}

Carter, B., Dorohoff, M., Good, M., Lew, J., Williams II, R., “Mechanical Design and Modeling of an Omni-directional RoboCup Player,” The 2001 RoboCup International Symposium, 2001.

\section{FIELDS OF STUDY}

Major Field: Mechanical Engineering

Studies in Vehicle Dynamics - Dr. Dennis Guenther 
TABLE OF CONTENTS

Page

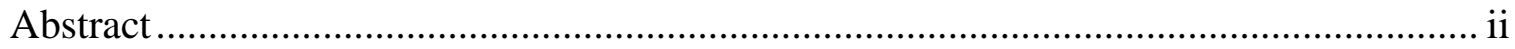

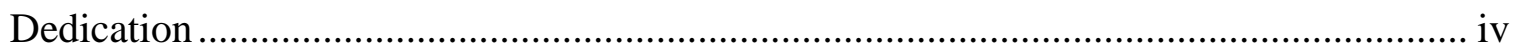

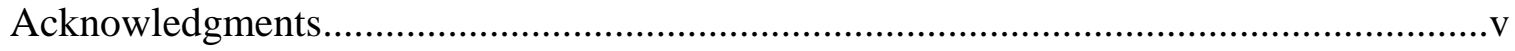

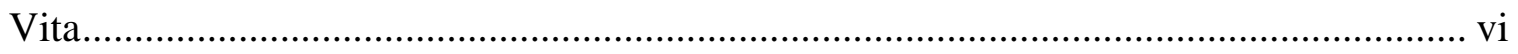

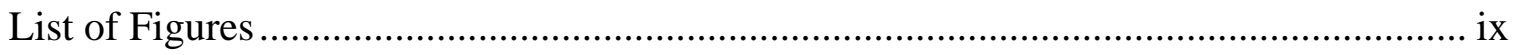

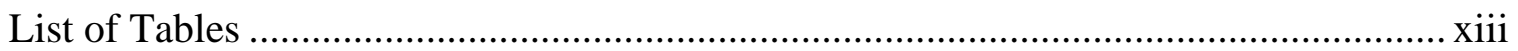

Chapters:

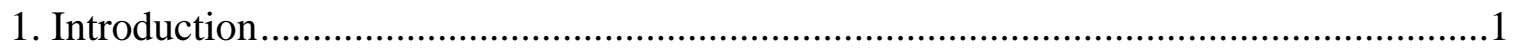

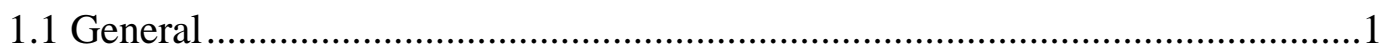

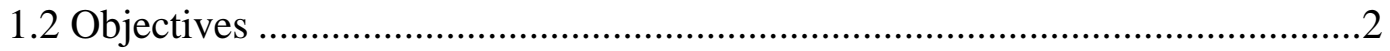

1.3 Thesis Overview ………………………….................................................

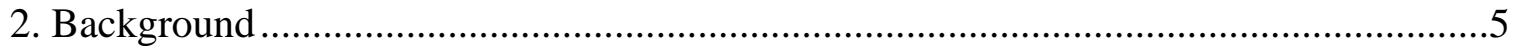

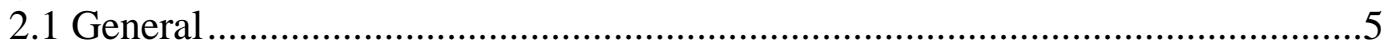

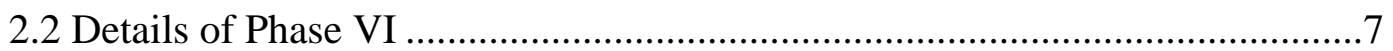

2.2.1 Test vehicles and configurations.......................................................

2.2.2 Instrumentation ............................................................................

2.2.3 Test maneuvers .............................................................................

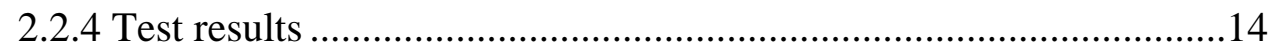

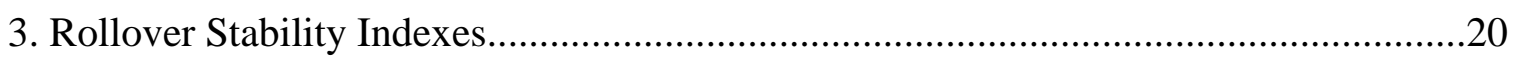

3.1 Introduction ...........................................................................................20

3.2 Overview of Rollover Stability Indexes ……………....................................20

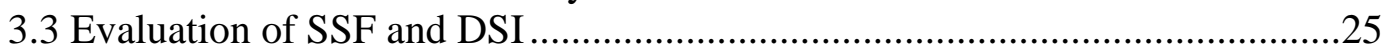

3.4 Modification of SSF and DSI ………………….....................................29

3.5 Conclusions and Recommends of the Rollover Stability Index Study .............36

4. Evaluation of the Field Test Data .............................................................................38

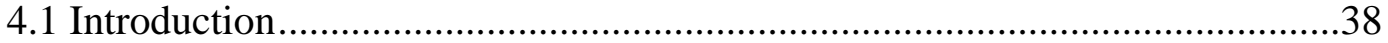

4.2 Accuracy and Repeatability of the Collected Data .............................................38

4.2.1 Commanded steering inputs (handwheel position and rate)..............39

4.2.2 Initial handwheel angle prior to the maneuver ...................................42 
4.2.3 Environmental effects .....................................................................42

4.2.4 Vehicle Dynamic Area (VDA) test surface ........................................43

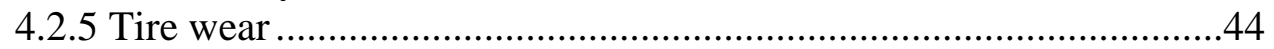

4.2.6 Vehicle age and wear: worn or damaged suspension components ...46

4.2.7 Test-to-test variability .......................................................................46

4.3 Common Vehicle Asymmetries ..................................................................50

4.4 Understeer and Roll Gradients....................................................................51

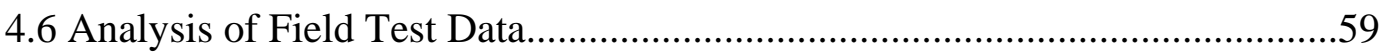

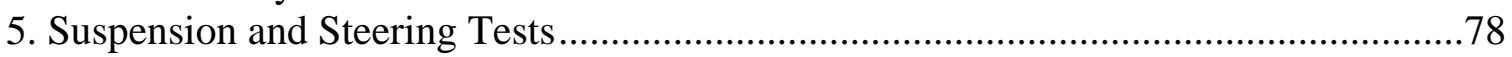

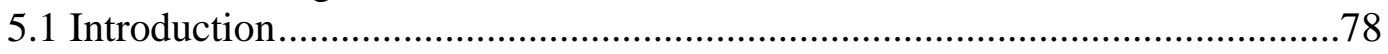

5.2 Description of Suspension and Steering Tests..............................................78

5.3 Results of the Suspension and Steering Tests................................................81

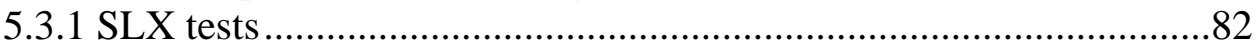

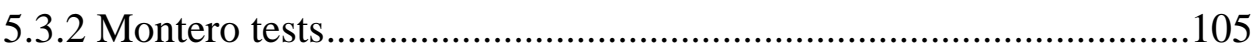

5.4 Physical Suspension Asymmetries ..............................................................127

6. Vehicle Dynamics Simulation Using CarSim ${ }^{\mathrm{TM}}$........................................................129

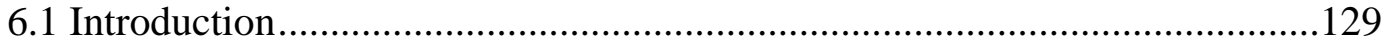

6.2 Validity of CarSim ${ }^{\mathrm{TM}}$..............................................................................129

6.3 The Vehicle Model Created in $\mathrm{CarSim}^{\mathrm{TM}}$....................................................130

6.4 Simulation Results .....................................................................................135

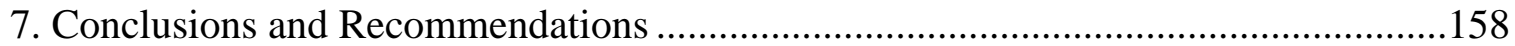

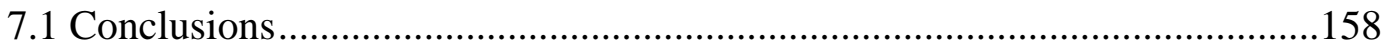

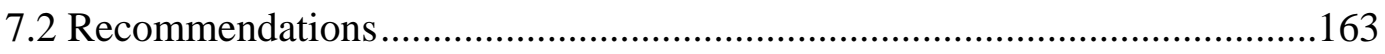

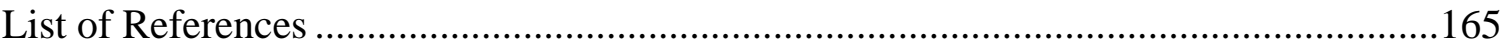

Appendix: Miscellaneous Equations and CarSim ${ }^{\mathrm{TM}}$ Parsfile ...........................................169 


\section{LIST OF FIGURES}

Figure

$\underline{\text { Page }}$

Figure 2.1: Slowly Increasing Steer Maneuver Description ....................................... 10

Figure 2.2: J-turn Maneuver Description............................................................... 11

Figure 2.3: Road Edge Recovery Maneuver Description ..............................................13

Figure 3.1: Forces Acting to Roll Over a Vehicle ........................................................22

Figure 3.2: Example of Dynamic Stability Index ....................................................24

Figure 4.1: Comparison of SLX Response at $\approx 39 \mathrm{mph}$, Nominal Load, Left Steer

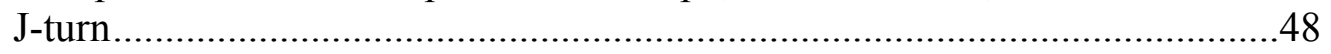

Figure 4.2: Differences in Roll Angle........................................................................49

Figure 4.3: SLX, Slowing Increasing Steer Maneuver ..............................................55

Figure 4.4: Montero, Slowing Increasing Steer Maneuver...........................................56

Figure 4.5: Aerostar, Slowing Increasing Steer Maneuver .........................................57

Figure 4.6: Aerostar Roll Gain, Slowing Increasing Steer Maneuver .............................58

Figure 4.7: SLX, Nominal Load, J-turn at Onset of TWL Left Steer at $39.9 \mathrm{mph}$, Right Steer at $50.2 \mathrm{mph}$

Figure 4.8: SLX, Nominal Load, J-turn at Common Entrance Speed Left Steer at $44.5 \mathrm{mph}$, Right Steer at $44.2 \mathrm{mph}$

Figure 4.9: SLX, Maximum Occupancy, J-turn at Onset of TWL Left Steer at $34.6 \mathrm{mph}$, Right Steer at $61.1 \mathrm{mph}$

Figure 4.10: SLX, Maximum Occupancy, J-turn at Common Entrance Speed Left Steer at $36.9 \mathrm{mph}$, Right Steer at $37.0 \mathrm{mph}$ 
Figure 4.11: SLX, Nominal Load, Road Edge Recovery at Onset of TWL Left-Right Steer at $39.5 \mathrm{mph}$, Right-Left Steer at $36.4 \mathrm{mph}$.

Figure 4.12: SLX, Nominal Load, Road Edge Recovery at Common Entrance Speed Left-Right Steer at $36.4 \mathrm{mph}$, Right-Left Steer at $36.4 \mathrm{mph}$

Figure 4.13: Montero, Nominal Load, J-turn at Common Entrance Speed Left Steer at $60.3 \mathrm{mph}$, Right Steer at $59.9 \mathrm{mph}$

Figure 4.14: Montero, Maximum Occupancy, J-turn at Onset of TWL Left Steer at $60.1 \mathrm{mph}$, Right Steer at $30.6 \mathrm{mph}$

Figure 4.15: Montero, Maximum Occupancy, J-turn at Common Entrance Speed Left Steer at $36.1 \mathrm{mph}$, Right Steer at $35.6 \mathrm{mph}$

Figure 4.16: Montero, Nominal Load, Road Edge Recovery at Onset of TWL Left-Right Steer at $32.7 \mathrm{mph}$, Right-Left Steer at $40.6 \mathrm{mph}$.

Figure 4.17: Montero, Nominal Load, Road Edge Recovery at Common Entrance Speed Left-Right Steer at $35.1 \mathrm{mph}$, Right-Left Steer at $35.6 \mathrm{mph}$

Figure 4.18: Aerostar, Nominal Load, J-turn at Common Entrance Speed Left Steer at $61.6 \mathrm{mph}$, Right Steer at $61.6 \mathrm{mph}$ .71

Figure 4.19: Aerostar, Maximum Occupancy, J-turn at Onset of TWL Left Steer at $52.1 \mathrm{mph}$, Right Steer at $59.7 \mathrm{mph}$

Figure 4.20: Aerostar, Maximum Occupancy, J-turn at Common Entrance Speed Left Steer at $37.1 \mathrm{mph}$, Right Steer at $37.0 \mathrm{mph}$

Figure 4.21: Aerostar, Maximum Occupancy, Road Edge Recovery at Onset of TWL Left-Right Steer at $46.5 \mathrm{mph}$, Right-Left Steer at $40.8 \mathrm{mph}$.

Figure 4.22: Aerostar, Maximum Occupancy, Road Edge Recovery at Common Entrance Speed, Left-Right Steer at $37.5 \mathrm{mph}$, Right-Left Steer at $36.6 \mathrm{mph}$ .75

Figure 5.1: SLX Front Suspension Spring Rate................................................... 86

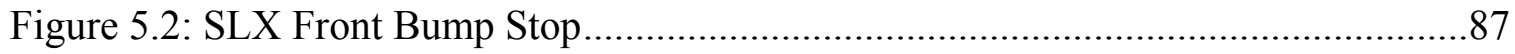

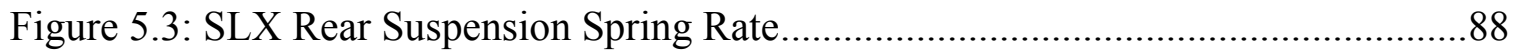

Figure 5.4: Sketch of SLX Rear Bump Stop (side view) ......................................... 88

Figure 5.5: SLX Front Overall Roll Stiffness .91 
Figure 5.6: SLX Rear Overall Roll Stiffness ........................................................92

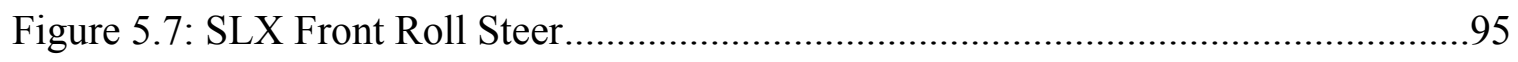

Figure 5.8: SLX Front Wheel Lateral Force Steer Compliance ....................................97

Figure 5.9: SLX Front Steering Compliance .........................................................100

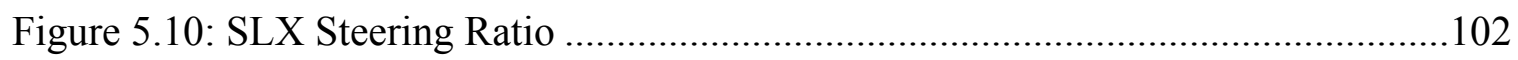

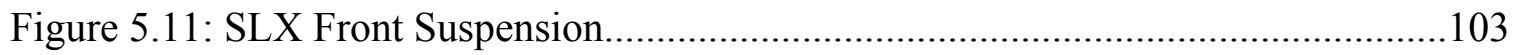

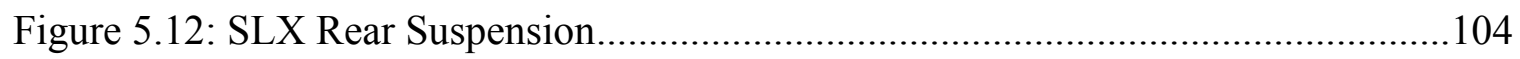

Figure 5.13: Montero Front Suspension Spring Rate .............................................109

Figure 5.14: Montero Rear Suspension Spring Rate ...............................................111

Figure 5.15: Montero Front Overall Roll Stiffness...................................................113

Figure 5.16: Montero Rear Overall Roll Stiffness.......................................................114

Figure 5.17: Montero Front Roll Steer .................................................................116

Figure 5.18: Montero Front Wheel Lateral Force Steer Compliance ............................118

Figure 5.19: Montero Front Steering Compliance .....................................................120

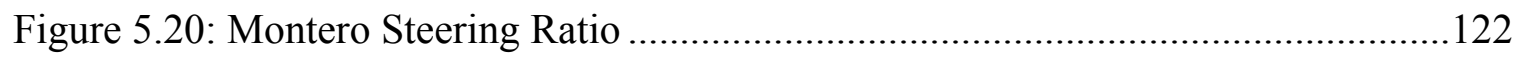

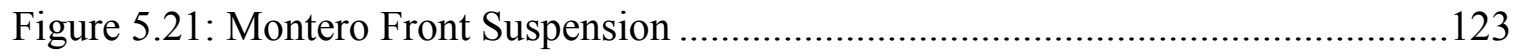

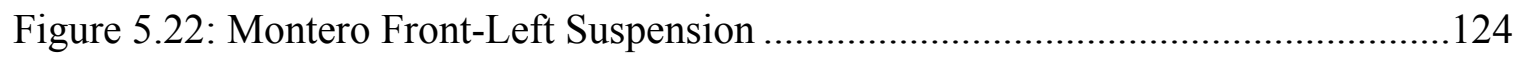

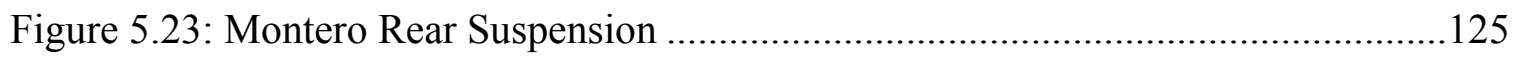

Figure 5.24: Montero Rear-Left Suspension ...................................................... 126

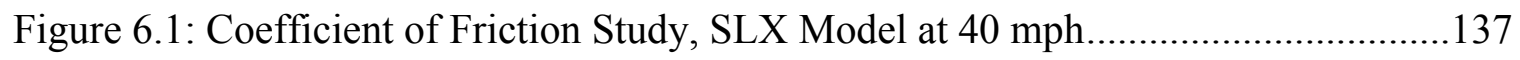

Figure 6.2: Big SUV Baseline Model at 40, 50, and $60 \mathrm{mph}$.....................................138

Figure 6.3: SLX Model: Y vs. X Trajectory, Effect of the Lateral CG Offset ................144

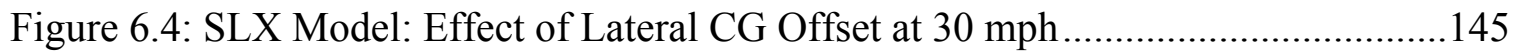


Figure 6.5: SLX Model: Effect of Lateral CG Offset at $40 \mathrm{mph}$ 146

Figure 6.6: SLX Model: Effect of Asymmetric Suspension at $30 \mathrm{mph}$, No Lateral CG Offset

Figure 6.7: SLX Model: Effect of Asymmetric Suspension at $60 \mathrm{mph}$, No Lateral CG Offset

Figure 6.8: SLX Model: Effect of Asymmetric Suspension Parameters and Lateral CG Offset at $30 \mathrm{mph}$

Figure 6.9: SLX Model: Effect of Asymmetric Suspension Parameters and Lateral CG Offset at $40 \mathrm{mph}$ 150

Figure 6.10: CarSim ${ }^{\mathrm{TM}}$ Default Shock Absorber Response.

Figure 6.11: Shock Absorber Study, SLX Model, Default Damping Force for Comparison

Figure 6.12: Shock Absorber Study, SLX Model, Decrease the Default Damping Force by $10 \%$

Figure 6.13: Shock Absorber Study, SLX Model, Increase the Default Damping Force by $10 \%$

Figure 6.14: Shock Absorber Study, SLX Model, Increase the Default Damping Force by $20 \%$

Figure 6.15: Shock Absorber Study, SLX Model, Increase the Front Default Damping Force by $10 \%$ 156

Figure 6.16: Shock Absorber Study, SLX Model, Increase the Rear Default Damping Force by $10 \%$ 


\section{LIST OF TABLES}

$\underline{\text { Table }}$

$\underline{\text { Page }}$

Table 2.1: Test Vehicle Weight, C.G. Location, and Mass Moments of Inertia .15

Table 2.2: Minimum Maneuver Entrance Speeds (mph) Required to Produce

Two-Wheel Lift .16

Table 2.3: J-Turn Maneuver Entrance Speed Versus Two-Wheel Lift Repeatability Check

Table 2.4: Road Edge Recovery Maneuver Entrance Speed Versus Two-Wheel Lift Repeatability Check

Table 3.1: Analysis of DSI/SSF as TWL Predictors (Phase VI Data).............................28

Table 3.2: Analysis of the Modified DSI/SSF Metrics as TWL Predictors........................33

Table 3.3: Accuracy of Rollover Stability Indexes....................................................34

Table 4.1: Handwheel Controller Angles (deg) and Rates (deg/sec)...............................41

Table 4.2: Understeer Gradient, Roll Gradient, and Maximum Lateral Acceleration;

Slowly Increasing Steer Maneuver

Table 5.1: SLX Suspension and Steering Tests

Table 5.2: SLX Suspension Test Summary 83-85

Table 5.3: Montero Suspension and Steering Tests .105

Table 5.4: Montero Suspension Test Summary 106-108

Table 6.1: Vehicle Parameters of Actual Vehicles and CarSim ${ }^{\mathrm{TM}}$ Models .132

Table 6.2: Shock Absorber Study .143 


\section{CHAPTER 1}

\section{INTRODUCTION}

\subsection{General}

The majority of vehicle dynamic models and simulations assume that a vehicle is symmetrical about the longitudinal axis (defined by SAE as the X-axis), and that a vehicle responds symmetrically to identical right versus left steer maneuvers. However, during the National Highway Traffic Safety Administration’s (NHTSA) research on light vehicle dynamic rollover propensity, field tests have shown several vehicles to have response asymmetries with respect to right versus left steer maneuvers. The test maneuvers have been well developed, are well defined, repeatable given the same driver inputs and environmental conditions, and are claimed to represent actual severe maneuvers a driver may reproduce during accident avoidance.

Some of these response asymmetries may be a result of vehicle lateral center of gravity (CG) offset, steering asymmetries, and/or suspension asymmetries, but further investigation is necessary to fully explain the response asymmetries. Furthermore, asymmetrical vehicle characteristics may support or contradict the asymmetrical vehicle responses observed. For example, a static CG offset toward the driver side will increase the energy needed to roll the vehicle to the right (clockwise rotation) during a left steer maneuver, which reduces the likelihood of rollover. However, the CG offset will 
compensate for the normal lateral weight transfer occurring in the turn, which keeps the vertical tire loads more balanced. Given that a balanced pair of tires will produce more lateral grip than an unbalanced pair, the vehicle will achieve more lateral acceleration, and therefore will be more prone to rollover. Thus the issue of asymmetrical vehicle response is a complicated cause and effect investigation.

This research will focus on the vehicle test data gathered at NHTSA's Vehicle Research and Test Center (VRTC) during Phase VI of NHTSA's Light Vehicle Rollover Research Program [1]. The objective of Phase VI was to assess the rollover resistance of a broad range of twenty-six light vehicles (passenger cars, sport utility vehicles (SUVs), light trucks, and minivans). Of the vehicles tested during Phase VI, three vehicles exhibited significant response asymmetries. These vehicles are the 1996 Acura SLX, 1995 Mitsubishi Montero, and the 1993 Ford Aerostar.

\subsection{Objectives}

The major objectives of this research were to:

1. Evaluate selective rollover stability indexes using the Phase VI data.

2. Analyze the measurements taken (roll angle, lateral acceleration, handwheel angle, etc.) during Phase VI’s vehicle field tests.

3. Determine what vehicles responded asymmetrically in Phase VI tests.

4. Identify any vehicle components/characteristics, such as steering system or suspension asymmetries, that may cause the asymmetric response.

5. Use vehicle dynamics computer simulation to evaluate the sensitivity of vehicle response to vehicle asymmetric characteristics. 


\subsection{Thesis Overview}

This thesis consists of seven chapters. This first chapter provides a basic introduction to the study, the objectives of the study, and an overview of the thesis.

The second chapter includes background information on the rollover research by NHTSA, in particular Phase VI of its Light Vehicle Rollover Research Program ("Phase VI”), where the vehicle field test data used in this thesis was collected. A detailed description of the test vehicles and configurations, instrumentation, test maneuvers, and the test results of Phase VI is also given.

An overview and an evaluation of rollover stability indexes are presented in the third chapter. This includes an analysis on modifying two of the indexes to better predict rollover instability.

The fourth chapter provides an evaluation of the field tests in Phase VI. This includes a discussion of the accuracy and repeatability of the collected data, common vehicle asymmetries, vehicle understeer and roll gradients, and an analysis of the field test data.

The fifth chapter accomplishes the fourth objective of this research, which is to identify any vehicle components/characteristics, such as steering system or suspension asymmetries that may cause vehicle asymmetric response. A description of the suspension and steering tests performed along with the results are given in this chapter.

The sixth chapter accomplishes the fifth objective of this research, which is to evaluate the sensitivity of vehicle response to vehicle asymmetric characteristics. The vehicle dynamic simulator used in this evaluation is introduced along with its validity, the vehicle model created, and the simulation results. 
The seventh and final chapter presents the conclusions of this research along with recommendations for future work in this area. 


\section{CHAPTER 2}

\section{BACKGROUND}

\subsection{General}

For over thirty years, NHTSA has been investigating the dynamic rollover propensity of light vehicles (vehicles that have a gross vehicle weight rating (GVWR) of 10,000 pounds or less, excluding motorcycles [2]). Efforts since 1997 include Phase I-A up to Phase VI of NHTSA's Light Vehicle Rollover Research Program, with additional phases yet to be published. The objectives of these phases are as follows:

I-A: Initial, exploratory study of using test track maneuvers to quantify on-road, untripped rollover propensity [2]

I-B: Investigate the effects of driver variability, outrigger, and fuel level on test results; implement the use of a programmable steering controller; and select maneuvers for Phase II testing [3]

II: Test a broad range of light vehicles using the test maneuvers and procedures developed during Phase I-A and I-B; characterize the on-road, untripped rollover 
propensities of the vehicles tested; compare the test results with the vehicles' static and dynamic rollover metrics; improve upon the test maneuvers and procedures used [4]

III-A: Determine when to initiate handwheel steering reversal during fishhook maneuver as to maximize the severity of the maneuver with respect to two-wheel lift/rollover [5]

III-B: Implement automated pulse braking in the J-turn maneuver [6]

IV: Evaluate maneuvers to be used to assess on-road, untripped rollover resistance of light vehicles [7]

V: Finalize the test methodology and procedures to be used during Phase VI and investigate the effect of different outrigger designs, ambient temperatures, and test surfaces on vehicle response (yet to be published, see [1] for details)

VI: Assess the rollover resistance of a broad range of twenty-six light vehicles [1]

These efforts serve to meet the Transportation Recall Enhancement, Accountability, and Documentation (TREAD) Act of 2000 to develop a dynamic test on passenger vehicle rollovers for the purposes of a consumer information program in which the resulting rollover resistance ratings will be part of NHTSA's New Car Assessment Program (NCAP). 
The main driving force for the TREAD Act of 2000 and NHTSA's recent rollover research is the fact that a large number of Americans are killed each year in rollover crashes. According to the report, “Traffic Safety Facts 2001” [8], 9866 fatal light vehicle rollover accidents occurred in 2001, which represents $20.5 \%$ of the total fatal light vehicle accidents. Of all the fatal SUV accidents, $35.2 \%$ were rollovers, as were $15.7 \%$ for passenger cars, $25.1 \%$ for pickup trucks, and $19.2 \%$ for vans. The percentages for fatalities in single vehicle crashes were even higher. Consider the following statistics based on the Fatality Analysis Reporting System (FARS). In 2001, 10,157 people were killed as occupants in light vehicle rollover crashes, of which 8,424 were killed in single vehicle rollovers crashes. The statistics show that of the 2,279 occupants killed in SUV single vehicle crashes, $76 \%$ were rollover crashes. Other light vehicle data are $45 \%$ for passenger cars, $61 \%$ for vans, and $60 \%$ for pickup trucks [9].

\subsection{Details of Phase VI}

As mentioned earlier, the objective of Phase VI of NHTSA's Light Vehicle Rollover Research Program was to assess the rollover resistance of a broad range of twenty-six light vehicles. As determined in earlier phases, two maneuvers (J-turn and fishhook, also referred to as road edge recovery) were used to evaluate the vehicles in both nominal and maximum occupancy load configurations. The remainder of this chapter presents in detail the test vehicles and configurations, instrumentation, test maneuvers, and the test results of Phase VI. For additional information on Phase VI, refer to [1]. 


\subsubsection{Test vehicles and configurations}

The twenty-six light vehicles evaluated in Phase VI consisted of nine SUVs, six pickup trucks, five minivans, and six passenger cars. The majority of the vehicles were used vehicles. To ensure performance similar to manufacturer design, the suspension components of the used vehicles were refurbished along with any other components that did not meet the manufacturers standards. Also, a four-wheel alignment was performed before testing.

All of the tires were new, inflated to vehicle manufacturer recommended pressure, and recommended by the vehicle manufacturer as $\mathrm{OE}$ equipment or equivalent. Prior to formal testing, the tires underwent a break-in procedure to wear away mold sheen and bring them up to operating temperatures. Since the effect of tire wear can have a strong influence on vehicle response, the tires were changed after each test series, e.g., one test series consisted of both left and right steer J-turn tests in the nominal load condition. The effect of tire wear will be discussed in Chapter 4.

The two vehicle load configurations were nominal load and maximum occupancy. The nominal load configuration consisted of the driver, instrumentation, steering machine, titanium outriggers (attached to the front and rear bumper attachment points), and a full gas tank. In addition to the nominal load configuration, maximum occupancy also consisted of water dummies in each seating position, excluding the front seats. In the case of the pickup trucks where no rear seats existed, a comparable loading condition to that of an SUV was used (water dummies were placed in the pickup truck bed close to the cab). 


\subsubsection{Instrumentation}

Each vehicle was similarly instrumented with sensors, data acquisition system, and a programmable steering machine. The following data were measured (by the given sensor): handwheel angle (angle encoder); brake pedal force (load cell); longitudinal, lateral, and vertical accelerations along with roll, yaw, and pitch rates (multi-axis inertial sensing system); left and right side vehicle ride height (ultrasonic distance measuring system); vehicle speed (radar speed sensor); and wheel lift (analog displacement measuring system). Appropriate measures were taken to correct for any CG offset placement of the accelerometers and the effect of roll angle. All data were sampled at $200 \mathrm{~Hz}$, and signal conditioning consisted of amplification, anti-alias filtering, and digitizing analog signals.

\subsubsection{Test maneuvers}

The test vehicles were evaluated with a characterization maneuver (slowly increasing steer) and two rollover resistance maneuvers (J-turn and road edge recovery). The slowly increasing steer maneuver was based on the "Constant Speed, Variable Steer" test defined in SAE J266 [10], and the data output from the maneuver was used to determine the handwheel input magnitudes for the J-turn and road edge recovery maneuvers. Hence, it characterized the lateral response of the vehicle, but did not evaluate the vehicle's rollover resistance. The maneuver was performed at $50 \mathrm{mph}$ in both the left and right steer directions. At time zero, the handwheel angle was linearly increased from $0^{\circ}$ to $270^{\circ}$ at a rate of $13.5^{\circ} / \mathrm{sec}$ and then held constant at $270^{\circ}$ for two seconds, as shown in Figure 2.1. 


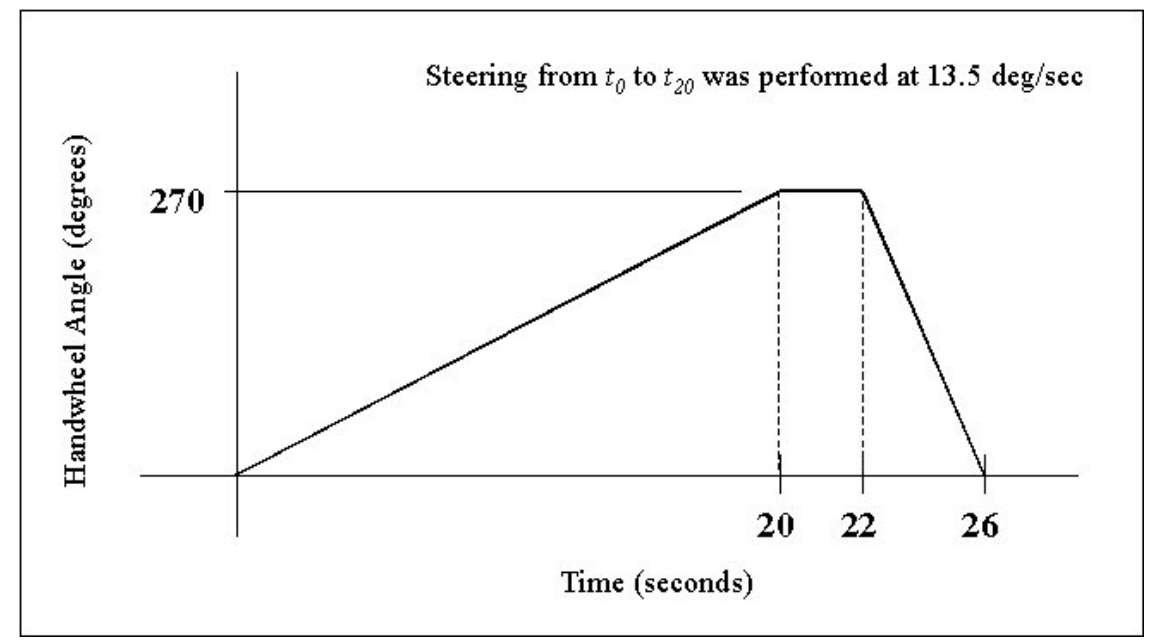

Figure 2.1: Slowly Increasing Steer Maneuver Description [1]

The J-turn is a basic, single step steer input. To begin the maneuver, the vehicle was driven in a straight line at a speed slightly greater than the desired entrance speed. The driver released the throttle, and when at the target speed, the steering machine initiated the handwheel commands described in Figure 2.2. Following completion of the handwheel ramp, handwheel position was maintained for four seconds and then returned to zero. The J-turn handwheel magnitudes, shown as 'A' in Figure 2.2, were calculated by multiplying the handwheel angle producing an average of $0.3 \mathrm{~g}$ in the slowly increasing steer maneuver by a scalar of 8.0. The handwheel rate was $1000^{\circ} / \mathrm{sec}$ for all test vehicles. 


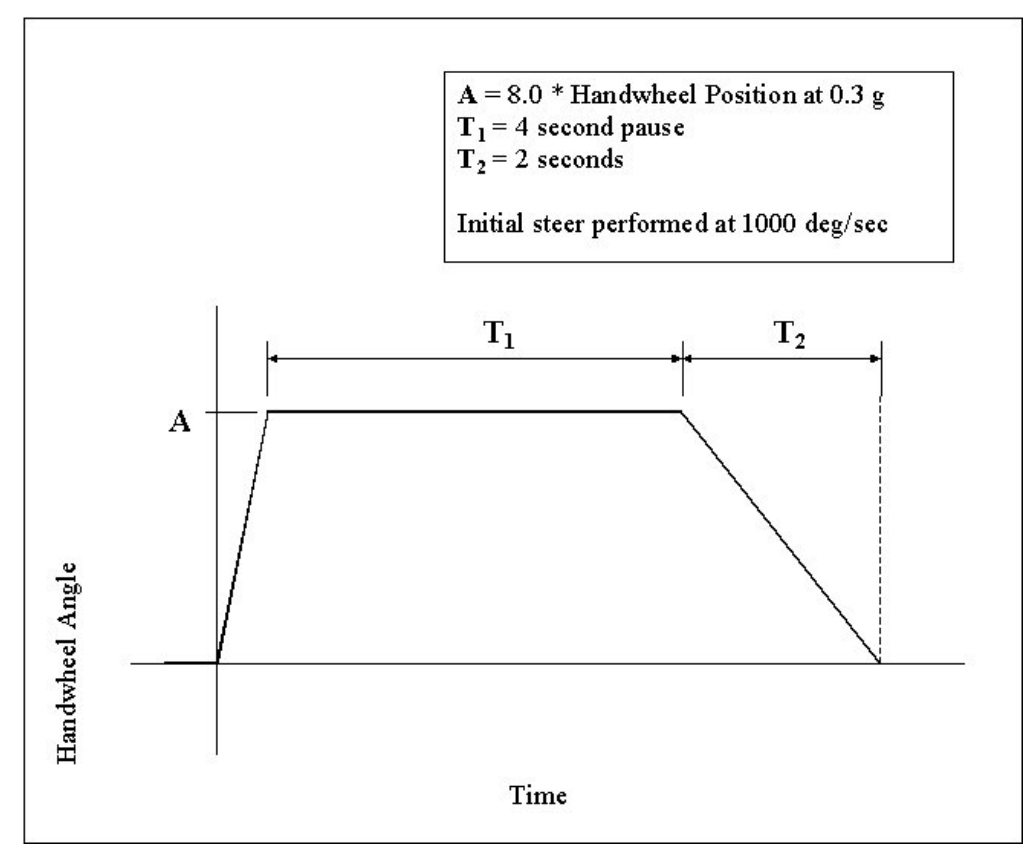

Figure 2.2: J-turn Maneuver Description [1]

The entrance speeds used in the J-turn maneuver ranged from 35 to $60 \mathrm{mph}$, and were increased in 5 mph increments until either two-wheel lift (TWL) occurred or the maximum entrance speed of $60 \mathrm{mph}$ was reached without TWL having occurred. If TWL was observed, a downward iteration of vehicle speed in $1 \mathrm{mph}$ increments was performed until lift was no longer detected. Once this lowest speed for TWL was isolated, two additional J-turns were performed at that speed to confirm TWL repeatability.

The road edge recovery maneuver is the same as NHTSA's roll rate feedback fishhook used in earlier rollover research phases. The phrase "road edge recovery” was coined because the handwheel inputs are claimed to represent the steering that a startled driver might use to regain lane position on a two-lane road after dropping two wheels off 
onto the shoulder. In addition, more people could easily relate to "road edge recovery" versus "fishhook". However, note that the road edge recovery maneuver is performed on a smooth uniform surface where the wheels never contact or drop off of the shoulder edge of the test surface.

To begin the maneuver, the vehicle was driven in a straight line at a speed slightly greater than the desired entrance speed. The driver released the throttle, and when at the target speed, the steering machine initiated the handwheel commands described in Figure 2.3. Following completion of the countersteer, handwheel position was maintained for three seconds and then returned to zero.

In a manner similar to that used for the J-turn, road edge recovery handwheel magnitudes were calculated by multiplying the handwheel angle producing an average of $0.3 \mathrm{~g}$ in the slowly increasing steer maneuver by a scalar of 6.5, as shown in Figure 2.3 as value ' $\mathrm{A}$ '. The timing of the countersteer is meant to maximize the severity of the maneuver. If a counter-clockwise initial steer was used (left steer), the countersteer following completion of the first handwheel ramp was to occur when the roll velocity of the vehicle was $1.5^{\circ} / \mathrm{sec}$ (considered a left-right steering road edge recovery maneuver). In similar fashion, the right-left steering road edge recovery maneuver's countersteer was to occur when the roll velocity of the vehicle was $-1.5^{\circ} / \mathrm{sec}$, as shown in Figure 2.3. The handwheel rates of the initial steer and countersteer were $720^{\circ} / \mathrm{sec}$ for all test vehicles. The nominal maneuver entrance speeds used in the road edge recovery maneuver ranged from 35 to $50 \mathrm{mph}$, and the procedure used to isolate the TWL speed was the same as in the J-turn tests. 


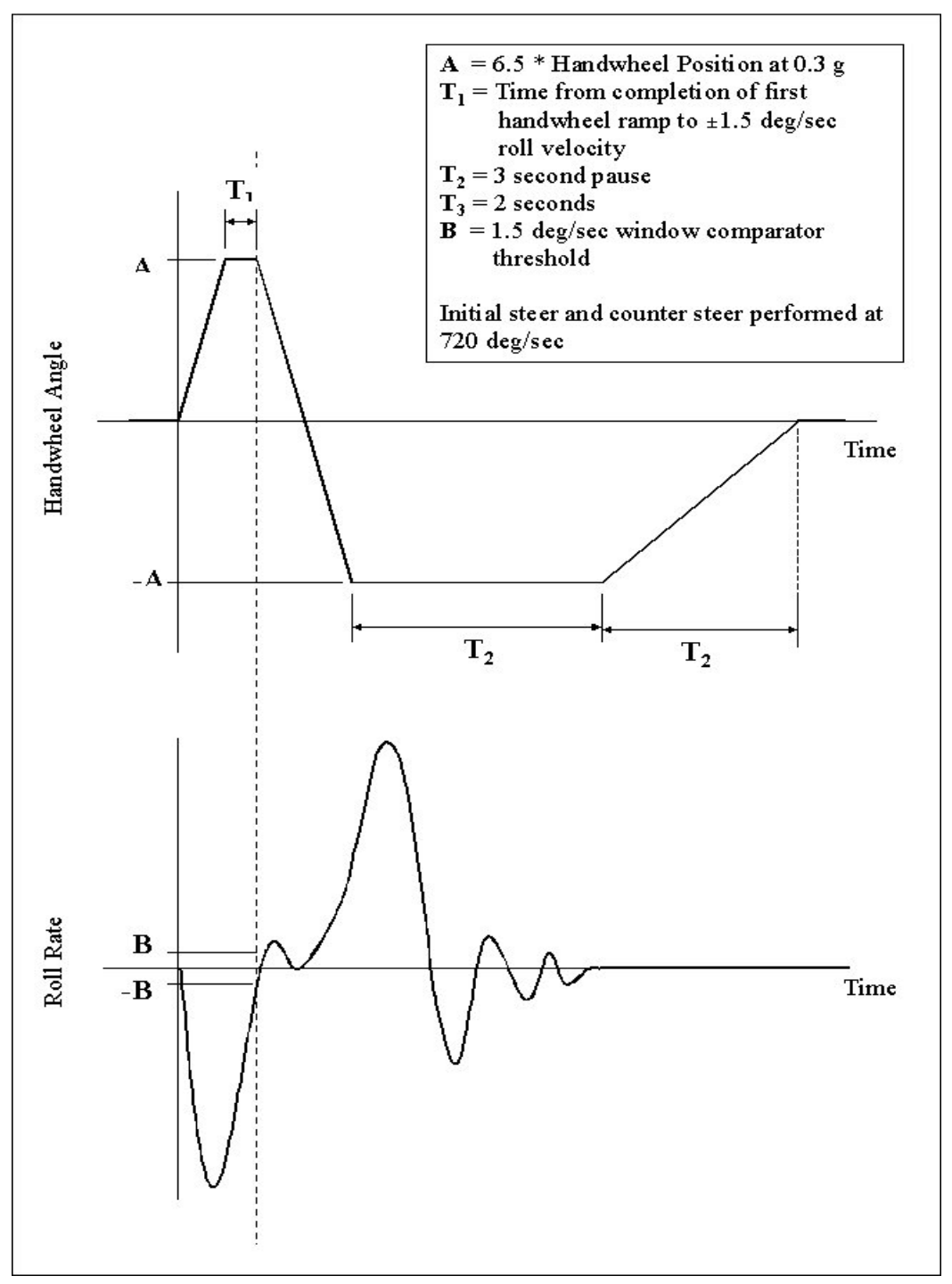

Figure 2.3: Road Edge Recovery Maneuver Description [1] 


\subsubsection{Test results}

A list of all the Phase VI vehicles, including the vehicle weight, CG location, static stability factor (SSF, discussed in Chapter 3), and mass moments of inertia is given in Table 2.1.

Two-wheel lift (TWL) is defined as at least two inches of simultaneous front and rear wheel lift for one side of a vehicle. TWL less than two inches was not considered. The minimum maneuver entrance speeds required to produce TWL are reported in Table 2.2, where ten of the twenty-six vehicles experienced TWL. Dashes indicate that TWL did not occur at or below the maximum test speed (50 mph for J-turn and $60 \mathrm{mph}$ for road edge recovery). Refer to [1] for explanations of tests that were not performed (TNP) or terminated early (ET). 


\begin{tabular}{|c|c|c|c|c|c|c|c|c|}
\hline \multirow[b]{2}{*}{ Vehicle } & \multirow{2}{*}{$\begin{array}{l}\text { Weight } \\
\text { (lbs) }\end{array}$} & \multicolumn{3}{|c|}{ C.G. } & \multirow[b]{2}{*}{ SSF } & \multicolumn{3}{|c|}{ Mass Moments of Inertia } \\
\hline & & $\begin{array}{l}\text { Longitudinal } \\
\text { (in) }\end{array}$ & $\begin{array}{l}\text { Height } \\
\text { (in) }\end{array}$ & $\begin{array}{l}\text { Lateral Offset } \\
\text { (in) }\end{array}$ & & $\begin{array}{c}\text { Pitch } \\
\left.\text { (ft-lb-sec }{ }^{2}\right)\end{array}$ & $\begin{array}{c}\text { Roll } \\
\left.\text { (ft-lb-sec }{ }^{2}\right)\end{array}$ & $\begin{array}{c}\text { Yaw } \\
\left.\text { (ft-lb-sec }{ }^{2}\right)\end{array}$ \\
\hline 1998 Honda CR-V & 3371 & 46.63 & 25.03 & -0.59 & 1.210 & 1826 & 431 & 1977 \\
\hline 1998 Chevrolet Tracker & 2625 & 39.87 & 24.26 & -0.84 & 1.131 & 959 & 262 & 1044 \\
\hline 1997 Jeep Cherokee Sport & 3684 & 44.95 & 26.38 & -0.91 & 1.102 & 1838 & 420 & 1993 \\
\hline 2001 Toyota 4Runner & 4239 & 48.57 & 27.07 & -1.17 & 1.098 & 2437 & 463 & 2544 \\
\hline 1996 Acura SLX & 4467 & 52.13 & 27.33 & -0.51 & 1.098 & 2758 & 572 & 2874 \\
\hline 2001 Ford Explorer XLS & 4446 & 51.38 & 27.10 & -1.45 & 1.085 & 2611 & 497 & 2685 \\
\hline 2001 Ford Explorer Sport & 4057 & 47.55 & 27.37 & -1.48 & 1.070 & 2161 & 466 & 2247 \\
\hline 2001 Chevrolet Blazer & 3998 & 49.10 & 26.63 & -1.00 & 1.025 & 2273 & 429 & 2384 \\
\hline 1995 Mitsubishi Montero & 4655 & 57.55 & 29.35 & -1.13 & 0.953 & 2859 & 581 & 2920 \\
\hline 1992 Ford F-150 & 4397 & 56.53 & 26.50 & -0.86 & 1.225 & 3638 & 479 & 3909 \\
\hline 1994 Chevrolet C1500 & 4273 & 57.22 & 26.33 & -1.06 & 1.219 & 3268 & 517 & 3579 \\
\hline 1997 Ford F-150 & 4438 & 57.02 & 26.92 & -0.86 & 1.212 & 3538 & 572 & 3774 \\
\hline 1995 Chevrolet K1500 & 4856 & 53.45 & 27.74 & -1.16 & 1.152 & 3645 & 548 & 3911 \\
\hline 1997 Ford Ranger 4x2 & 3228 & 44.02 & 25.12 & -1.85 & 1.136 & 1702 & 336 & 1821 \\
\hline 1997 Ford Ranger 4x4 & 3723 & 43.22 & 27.08 & -1.78 & 1.070 & 1922 & 382 & 2048 \\
\hline 1998 Plymouth Voyager & 3812 & 46.92 & 24.96 & -1.34 & 1.273 & 2417 & 581 & 2609 \\
\hline 1995 Ford Windstar GL & 3943 & 46.72 & 25.34 & -0.48 & 1.258 & 2757 & 593 & 2974 \\
\hline 1994 Dodge Caravan & 3616 & 47.03 & 25.82 & -0.93 & 1.182 & 2207 & 475 & 2377 \\
\hline 1995 Chevrolet Astro & 4422 & 50.83 & 29.01 & -1.11 & 1.126 & 2778 & 690 & 2938 \\
\hline 1993 Ford Aerostar & 3879 & 51.46 & 27.30 & -1.21 & 1.117 & 2263 & 537 & 2389 \\
\hline 2002 Chevrolet Corvette & 3361 & 51.45 & 17.61 & -0.55 & 1.749 & 1442 & 341 & 1637 \\
\hline 1992 Honda Civic LX & 2529 & 39.90 & 19.88 & -1.09 & 1.462 & 1212 & 257 & 1360 \\
\hline 1994 Ford Taurus & 3407 & 37.63 & 21.08 & -0.94 & 1.447 & 1845 & 363 & 2072 \\
\hline 1993 Chevrolet Caprice Classic & 4097 & 50.68 & 22.21 & -0.55 & 1.406 & 2718 & 453 & 3022 \\
\hline 1997 Chevrolet Metro & 2057 & 35.41 & 20.37 & -1.46 & 1.326 & 782 & 202 & 867 \\
\hline 1991 Chevrolet Cavalier & 2728 & 36.99 & 21.19 & -0.88 & 1.310 & 1333 & 271 & 1474 \\
\hline
\end{tabular}

Table 2.1: Test Vehicle Weight, C.G. Location, and Mass Moments of Inertia (Baseline, Sorted By SSF In Descending Order, Per Vehicle Class) [1] 


\begin{tabular}{|c|c|c|c|c|c|c|c|c|}
\hline \multirow{3}{*}{ Vehicle } & \multicolumn{4}{|c|}{ J-Turn (Maximum Test Speed of $60 \mathrm{mph}$ ) } & \multicolumn{4}{|c|}{ Road Edge Recovery (Maximum Test Speed of $50 \mathrm{mph}$ ) } \\
\hline & \multicolumn{2}{|c|}{ Nominal Load } & \multicolumn{2}{|c|}{ Maximum Occupancy } & \multicolumn{2}{|c|}{ Nominal Load } & \multicolumn{2}{|c|}{ Maximum Occupancy } \\
\hline & Left Steer & Right Steer & Left Steer & Right Steer & $\begin{array}{l}\text { Left-Right } \\
\text { Steering }\end{array}$ & $\begin{array}{c}\text { Right-Left } \\
\text { Steering }\end{array}$ & $\begin{array}{l}\text { Left-Right } \\
\text { Steering }\end{array}$ & $\begin{array}{c}\text { Right-Left } \\
\text { Steering }\end{array}$ \\
\hline 1998 Honda CR-V & -- & -- & $\begin{array}{l}55.2 \\
\text { (ET) }\end{array}$ & $\begin{array}{l}- \\
(\mathrm{ET})\end{array}$ & 42.4 & $\begin{array}{l}- \\
(\mathrm{ET})\end{array}$ & $\begin{array}{l}43.7 \\
\text { (ET) }\end{array}$ & TNP \\
\hline 1998 Chevrolet Tracker & -- & -- & -- & -- & -- & -- & 43.5 & 45.4 \\
\hline 2001 Toyota 4Runner* & -- & -- & -- & -- & -- & -- & 37.2 & 45.7 \\
\hline 1996 Acura SLX & 39.9 & 50.2 & 34.6 & -- & 39.5 & 36.4 & 35.3 & 35.9 \\
\hline $\begin{array}{l}2001 \text { Ford Explorer } \\
\text { XLS }\end{array}$ & -- & -- & -- & -- & -- & -- & 37.5 & -- \\
\hline 2001 Chevrolet Blazer & -- & -- & 52.4 & 49.0 & 40.4 & 39.6 & 34.9 & 34.3 \\
\hline $\begin{array}{l}1995 \text { Mitsubishi } \\
\text { Montero }\end{array}$ & -- & -- & -- & 30.6 & 32.7 & 40.6 & 34.0 & 29.7 \\
\hline 1997 Ford Ranger $4 \times 4$ & -- & -- & -- & $\begin{array}{l}60.9 \\
\text { (ET) }\end{array}$ & 48.8 & $\begin{array}{l}51.4 \\
\text { (ET) }\end{array}$ & \multicolumn{2}{|c|}{ TNP } \\
\hline 1995 Chevrolet Astro & -- & -- & -- & $\begin{array}{l}57.9 \\
\text { (ET) }\end{array}$ & -- & - & 34.5 & 36.2 \\
\hline 1993 Ford Aerostar & -- & -- & 52.1 & -- & 43.3 & 46.5 & 46.5 & 40.8 \\
\hline
\end{tabular}

*The electronic stability control (VSC) on the 2001 4Runner was disabled during all Phase VI tests.

Table 2.2: Minimum Maneuver Entrance Speeds (mph) Required to Produce Two-Wheel Lift [1] (ET): Early Termination, TNP: Test Not Performed

(Sorted by Baseline SSF in Descending Order, Per Vehicle Class) 
Ideally, the vehicle response should be the same given identical steering and speed inputs as well as surface and environmental conditions. Such is the case with modeling and computer simulation. However, in actual field tests, test-to-test variability can cause small response fluctuations. Even more so, when testing at a vehicle's TWL threshold speed (the maneuver entrance speed for which two-wheel lift may or may not occur), the likelihood of TWL is very high, but not guaranteed. Therefore, the results of Phase VI’s TWL repeatability evaluation, presented in Tables 2.3 (J-turn) and 2.4 (road edge recovery), should not be surprising. The combination of test-to-test variability and testing at TWL threshold speeds adds to the complexity of analyzing vehicle response, e.g., when comparing left versus right steer vehicle response. 


\begin{tabular}{|c|c|c|c|c|c|c|c|c|c|c|c|c|}
\hline \multirow{3}{*}{ Vehicle } & \multicolumn{6}{|c|}{ Nominal Load } & \multicolumn{6}{|c|}{ Maximum Occupancy } \\
\hline & \multicolumn{3}{|c|}{ Left Steer } & \multicolumn{3}{|c|}{ Right Steer } & \multicolumn{3}{|c|}{ Left Steer } & \multicolumn{3}{|c|}{ Right Steer } \\
\hline & Initial & $\begin{array}{c}1^{\text {st }} \\
\text { Repeat }\end{array}$ & $\begin{array}{c}2^{\text {nd }} \\
\text { Repeat }\end{array}$ & Initial & $\begin{array}{c}1^{\text {st }} \\
\text { Repeat }\end{array}$ & $\begin{array}{c}2^{\text {nd }} \\
\text { Repeat }\end{array}$ & Initial & $\begin{array}{c}1^{\text {st }} \\
\text { Repeat }\end{array}$ & $\begin{array}{c}2^{\text {nd }} \\
\text { Repeat }\end{array}$ & Initial & $\begin{array}{c}1^{\text {st }} \\
\text { Repeat }\end{array}$ & $\begin{array}{c}2^{\text {nd }} \\
\text { Repeat }\end{array}$ \\
\hline 1996 Acura SLX & 39.9 & $\begin{array}{c}39.5 \\
\text { (No TWL) }^{1}\end{array}$ & $\begin{array}{c}39.1 \\
\text { (No TWL) }^{1}\end{array}$ & -- & -- & -- & 34.8 & $\begin{array}{c}34.2 \\
\text { (No TWL) }\end{array}$ & 34.6 & -- & -- & -- \\
\hline $\begin{array}{l}2001 \text { Chevrolet } \\
\text { Blazer }\end{array}$ & -- & -- & -- & -- & -- & -- & 53.7 & $\begin{array}{c}52.6 \\
\text { (No TWL) }^{1}\end{array}$ & 52.4 & 49.0 & 49.5 & $\begin{array}{c}49.6 \\
\text { (No TWL) }^{2}\end{array}$ \\
\hline $\begin{array}{l}1995 \text { Mitsubishi } \\
\text { Montero }\end{array}$ & -- & -- & -- & -- & -- & -- & -- & -- & -- & 30.6 & 31.0 & 31.0 \\
\hline 1993 Ford Aerostar & -- & -- & -- & -- & -- & -- & -- & -- & -- & -- & -- & -- \\
\hline
\end{tabular}

$(\text { No TWL })^{1}=$ less than two inches of simultaneous two wheel lift was observed

$(\text { No TWL })^{2}=$ front wheel lift only, no rear wheel lift

Table 2.3: J-Turn Maneuver Entrance Speed Versus Two-Wheel Lift Repeatability Check [1] 


\begin{tabular}{|c|c|c|c|c|c|c|c|c|c|c|c|c|}
\hline \multirow{3}{*}{ Vehicle } & \multicolumn{6}{|c|}{ Nominal Load } & \multicolumn{6}{|c|}{ Maximum Occupancy } \\
\hline & \multicolumn{3}{|c|}{ Left-Right Steering } & \multicolumn{3}{|c|}{ Right-Left Steering } & \multicolumn{3}{|c|}{ Left-Right Steering } & \multicolumn{3}{|c|}{ Right-Left Steering } \\
\hline & Initial & $\begin{array}{c}1^{\text {st }} \\
\text { Repeat }\end{array}$ & $\begin{array}{c}2^{\text {nd }} \\
\text { Repeat }\end{array}$ & Initial & $\begin{array}{c}1^{\text {st }} \\
\text { Repeat }\end{array}$ & $\begin{array}{c}2^{\text {nd }} \\
\text { Repeat }\end{array}$ & Initial & $\begin{array}{c}1^{\text {st }} \\
\text { Repeat }\end{array}$ & $\begin{array}{c}2^{\text {nd }} \\
\text { Repeat }\end{array}$ & Initial & $\begin{array}{c}1^{\text {st }} \\
\text { Repeat }\end{array}$ & $\begin{array}{c}2^{\text {nd }} \\
\text { Repeat }\end{array}$ \\
\hline 1998 Honda CR-V & 42.4 & 42.5 & $\begin{array}{c}42.1 \\
\text { (No TWL) }\end{array}$ & -- & -- & -- & -- & -- & -- & -- & -- & -- \\
\hline $\begin{array}{l}1998 \text { Chevrolet } \\
\text { Tracker }\end{array}$ & -- & -- & -- & -- & -- & -- & 43.5 & $\begin{array}{c}43.4 \\
\text { (No TWL) }^{2}\end{array}$ & $\begin{array}{c}43.2 \\
\text { (No TWL) }^{2}\end{array}$ & 45.4 & $\begin{array}{c}45.9 \\
\text { (No TWL) }\end{array}$ & $\begin{array}{c}45.8 \\
\text { (No TWL) }^{1}\end{array}$ \\
\hline $\begin{array}{l}2001 \text { Toyota } \\
\text { 4Runner }\end{array}$ & -- & -- & -- & -- & -- & -- & 37.2 & 37.6 & 37.7 & 45.8 & 45.8 & 45.7 \\
\hline 1996 Acura SLX & 39.5 & 39.9 & 39.5 & -- & -- & -- & 36.0 & 35.3 & 35.7 & 35.9 & 36.5 & 36.5 \\
\hline $\begin{array}{l}\text { 2001 Ford } \\
\text { Explorer XLS }\end{array}$ & -- & -- & -- & -- & -- & -- & 38.6 & 37.5 & 39.2 & -- & -- & -- \\
\hline $\begin{array}{l}\text { 2001 Chevrolet } \\
\text { Blazer }\end{array}$ & 40.4 & 40.6 & 40.7 & 39.8 & 40.4 & 39.6 & 34.9 & 35.7 & $\begin{array}{c}36.8 \\
\text { (No TWL) }^{1}\end{array}$ & 35.2 & 34.3 & 34.3 \\
\hline $\begin{array}{l}1995 \text { Mitsubishi } \\
\text { Montero }\end{array}$ & 32.7 & 32.7 & $\begin{array}{c}32.9 \\
\text { (No TWL) }^{3}\end{array}$ & 40.6 & 40.7 & 41.0 & 34.6 & 34.6 & 34.0 & 29.7 & 30.1 & $\begin{array}{c}30.1 \\
\text { (No TWL) }^{3}\end{array}$ \\
\hline $\begin{array}{l}1997 \text { Ford Ranger } \\
4 \times 4\end{array}$ & 49.3 & 49.8 & 48.8 & -- & -- & -- & -- & -- & -- & -- & -- & -- \\
\hline $\begin{array}{l}1995 \text { Chevrolet } \\
\text { Astro }\end{array}$ & -- & -- & -- & -- & -- & -- & 34.9 & 34.6 & 34.5 & 36.2 & 36.8 & 36.8 \\
\hline $\begin{array}{l}1993 \text { Ford } \\
\text { Aerostar }\end{array}$ & 44.0 & 43.3 & $\begin{array}{c}43.0 \\
\text { (No TWL) }\end{array}$ & 46.5 & 47.0 & 46.7 & 47.2 & 46.6 & 46.5 & 40.8 & 41.1 & $\begin{array}{c}40.8 \\
\text { (No TWL) }^{2}\end{array}$ \\
\hline
\end{tabular}

$(\text { No TWL })^{1}=$ less than two inches of simultaneous two wheel lift was observed

$(\text { No TWL })^{2}=$ front wheel lift only, no rear wheel lift

$(\text { No TWL })^{3}=$ no front or rear wheel lift

Table 2.4: Road Edge Recovery Maneuver Entrance Speed Versus Two-Wheel Lift Repeatability Check [1] 


\section{CHAPTER 3}

\section{ROLLOVER STABILITY INDEXES}

\subsection{Introduction}

This chapter begins with an overview of both static and dynamic rollover stability indexes. Then an evaluation of two of the indexes is given based on vehicle field test data collected during Phase VI of NHTSA's Light Vehicle Rollover Research Program. Next is a discussion on the modification of these indexes to better represent the rollover instability threshold, along with an evaluation of the modified indexes. Since this investigation of rollover stability indexes considers but does not focus on asymmetric vehicle response, conclusions and recommendations of this index investigation are given at the end of this chapter instead of being included into the overall conclusion section of this thesis.

\subsection{Overview of Rollover Stability Indexes}

There are two types of rollover stability indexes: static (time-independent) and dynamic (time-dependent). Three common types of static indexes include the Static Stability Factor (SSF), Side Pull Ratio (SPR), and the Tilt Table Ratio (TTR). Countless dynamic indexes exist, influenced heavily by the dynamic onboard measurements (e.g. yaw rate, lateral acceleration, roll acceleration, etc.) available and the purpose (e.g. 
electronic stability controller, active suspension, etc.) of the index. The three static indexes mentioned are based on the estimation of the steady state lateral acceleration needed to cause a vehicle to roll over on a flat road. This lateral acceleration level represents a major, or first order, characteristic in vehicle rollover stability [11].

On May 25, 2000, NHTSA declared that, "after extensive research, NHTSA determined that a vehicle's 'static stability factor' is the most reliable indicator of rollover risk in single-vehicle crashes" [12]. NHTSA then incorporated SSF into a rollover resistance star rating, which can be found at NHTSA's website (http://www.nhtsa.dot.gov/NCAP). SSF has thus become the most popular rollover index. It (nominal SSF) is simply half of the vehicle's average track width (average of the front and rear) divided by the vehicle's CG height and is commonly referred to as " $t$ over 2h" [13]. The vehicle is considered to be a rigid body that approaches rollover instability as the lateral acceleration approaches SSF. This rigid body model neglects the effects of the suspension, roll angle of the sprung mass, and deformation of the tires, and it assumes sufficient tire-road friction such that the tires do not slide laterally. The model, including D'Alembert's force $\mathrm{M}^{*} \mathrm{a}_{\mathrm{y}}$, is shown in Figure 3.1. 


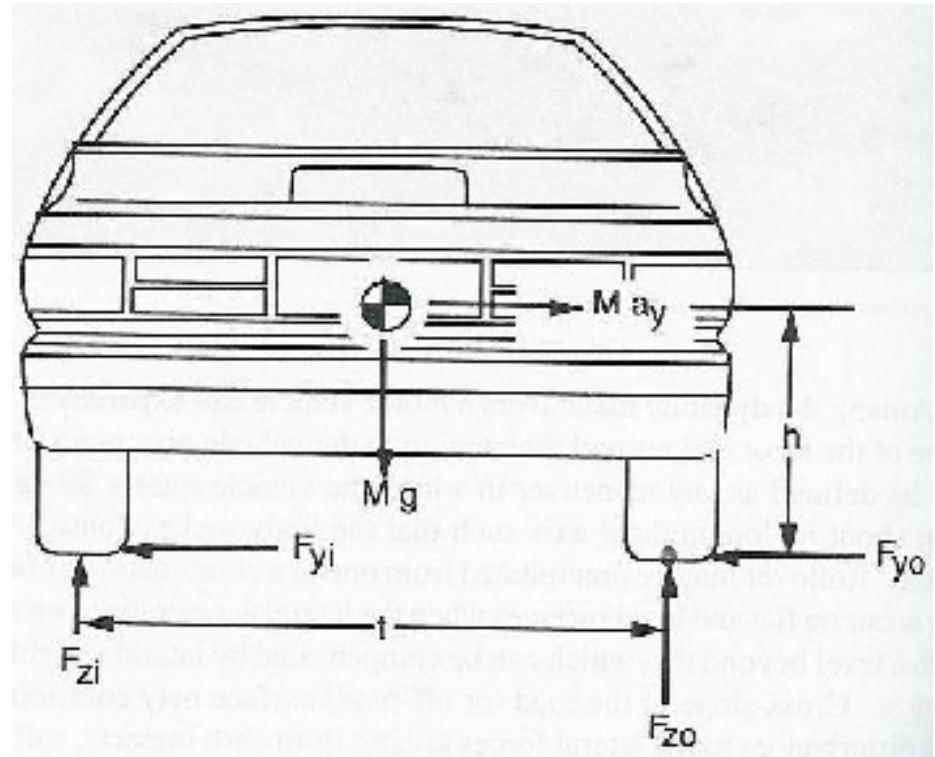

Figure 3.1: Forces Acting to Roll Over a Vehicle [13]

where:

M: entire mass of vehicle

$\mathrm{F}_{\mathrm{y}}$ : lateral tire force

g: gravitational constant $\mathrm{a}_{\mathrm{y}}$ : lateral acceleration

$\mathrm{F}_{\mathrm{z}}$ : vertical (normal) force on tire

h: total vehicle CG height

subscripts ' $i$ ' and 'o' denote inside and outside of turn, respectively.

Consider the moment about the center of contact for the outside tires (assume vehicle is in a steady turn, i.e., no roll acceleration, and the inside tires lose contact with the ground) and then the stabilizing moment is:

$$
\operatorname{Mg} \frac{t}{2}
$$

and the overturning (tip-up causing) moment is:

$$
M a_{y} h
$$


Setting the two moments equal to each other and modifying the equation yields:

$$
S S F: \frac{t}{2 h}=\frac{a_{y}}{g}
$$

So as the lateral acceleration (in g's) approaches "t over $2 \mathrm{~h}$," then the vehicle approaches rollover instability.

In an effort to better model the rollover dynamics of a vehicle, numerous dynamic indexes have been introduced, which account for the higher order effects of vehicle instability and allow for the implementation of active control systems. These dynamic indexes incorporate all of the dynamic test data (i.e., not just a single point) and represent the roll stability of the vehicle at each instance during a maneuver. An example of a dynamic index is given is Figure 3.2.

In 1998, Bernard, Shannan, and Vanderploeg built upon the static rigid model by accounting for the sprung mass body roll and the lateral deflection of the tires during a turning maneuver, which decreases the stabilizing moment given in SSF [14]. Gillespie also has a model that accounts for the roll of the body [13]. Heydinger and Howe introduced a Dynamic Stability Index (DSI) in 2000 that accounts for the roll moment of the sprung mass [15]. In addition to DSI, they suggested a modification of SSF to account for the lateral CG offset of a vehicle. In 2002, Hac considered many modifications to SSF, which include body roll, changes in half-track width due to suspension kinematics and lateral compliance of the tires and suspension, gyroscopic forces due to wheel rotation, and the effect of jacking forces [16]. Yang and Liu (2003) discussed three different dynamic indexes to be used in an active controller [17]. One of the indexes $\left(\mathrm{R}_{2}\right)$ mentioned by Yang and Liu is based on energy relationships, which 
according to Heydinger and Howe [15] are difficult to accurately model. Given the different nature of ' $R_{2}$ ', it was included in the index evaluation documented in Section 3.5.

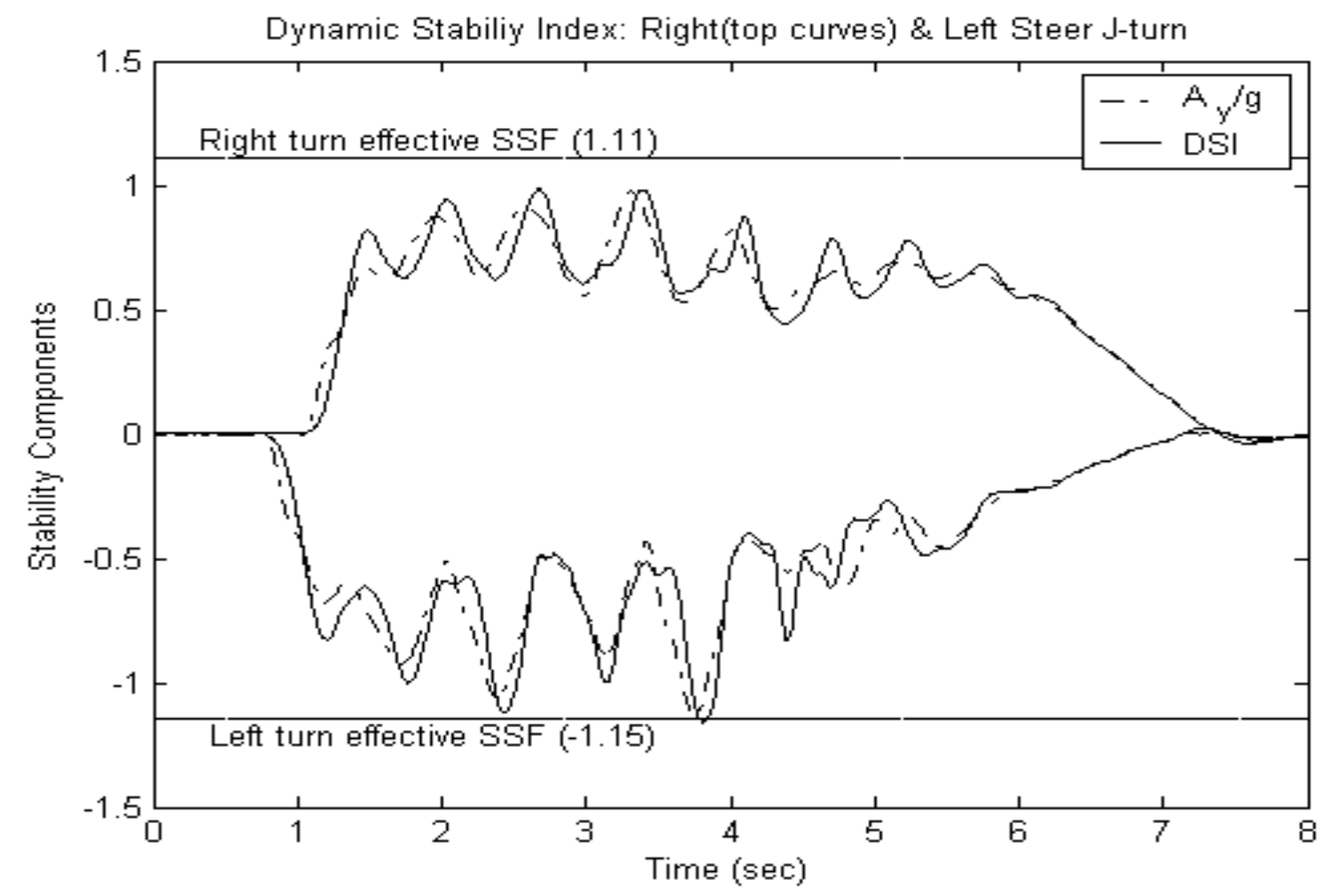

Figure 3.2: Example of Dynamic Stability Index 1996 Acura SLX, J-turn maneuver ( $\approx 44 \mathrm{mph})$ as described in Section 2.2.3

\subsection{Evaluation of SSF and DSI}

Of the dynamic rollover indexes mentioned, DSI was chosen for this study because of its simplicity and similarity to SSF. The DSI model is based on the SSF model with the addition of the roll moment of the sprung mass $\left(I_{x x s} \alpha\right)$, where the roll 
inertia of the sprung mass $\left(\mathrm{I}_{\mathrm{xxs}}\right)$ is assumed to be $80 \%$ of the entire vehicle roll inertia and ' $\alpha$ ' denotes roll acceleration. The tip-up causing moment in Equation 3.2 now becomes:

$$
M a_{y} h+I_{x x s} \alpha
$$

In [15], the SSF was modified to account for the lateral CG offset of a vehicle. The effective half-track width of the vehicle is:

$$
\left(\frac{t}{2}\right)_{\text {effective }}=\left(\frac{t}{2}\right)-C G_{-} \text {offset }
$$

where the sign of the CG offset is based on the SAE vehicle coordinate system.

Therefore, a CG offset toward the driver side of a vehicle would be a negative offset and would increase the effective half-track width during a left steer maneuver and decrease the effective half-track width during a right steer maneuver. The effective SSF for both the left and right steer maneuvers for the 1996 Acura SLX are shown in Figure 3.2. Now, the moment equation for this model is:

$$
M g\left(\frac{t}{2}\right)_{\text {effective }}=M a_{y} h+I_{x x s} \alpha
$$

Rearrange the moment equation to get:

$$
\text { Effective_SSF }: \frac{(t / 2)_{\text {effective }}}{h}=\frac{a_{y}}{g}+\frac{I_{x x s} \alpha}{M g h}: D S I
$$

Additional notes on DSI [15]:

- Theoretically, in any and all rollovers, DSI exceeds SSF.

- The first-order DSI is derived based on roll moments that are always lower than the "exact" roll moments a vehicle will experience in an actual dynamic maneuver.

- The DSI has to be greater than the SSF for some period of time in order to produce major two wheel lift (TWL). 
- As mentioned in the derivation of SSF, it is assumed that the tire contact patch provides adequate friction to achieve enough lateral force to cause vehicle wheel lift. However, during severe maneuvers, some vehicle-tire combinations might plow out and not provide enough lateral force to produce TWL.

- Some vehicles exhibit oscillatory roll response during severe maneuvers. DSI does not account for the oscillating energy transfer in the system (springs, shocks, tires, etc.).

Based on the derivation of DSI, the combination of SSF and DSI ("the metrics") should predict vehicle rollover (when the stabilizing moment is less than the tip-up causing moment). As mentioned in Chapter 2, NHTSA's objective in Phase VI was to determine the rollover resistance of a broad range of vehicles, which involved determining the minimum maneuver entrance speed needed to produce TWL. However, TWL does not necessarily result in rollover. Therefore, if during a severe maneuver a vehicle experiences TWL, the metrics should not be expected to predict TWL —unless for that particular case, TWL will result in vehicle rollover. Given this, a better validation test of the metrics would be to consider the field tests where the vehicle outriggers touched the ground, i.e., the vehicle would have rolled over in the absence of the outriggers.

However, if it is desired to use the metrics to predict TWL (which is assumed throughout this analysis), then one must determine a threshold value for DSI/SSF so that [DSI/SSF $>$ (threshold value)] will predict TWL. Currently, if DSI/SSF $>1$, major TWL and vehicle rollover is expected. 
Based on the Phase VI data, ten vehicles experienced TWL. The majority of the field tests for those ten vehicles were considered, for a total of 514 tests, as shown in Table 3.1. The metrics (DSI/SSF) predicted TWL (column 6) 34 out of the 166 tests that produced TWL and did not predict or "underestimated" TWL in the remaining 132 cases. The metrics predicted TWL (column 7) 23 times in which TWL did not occur (“overestimated" TWL). 


\begin{tabular}{|c|c|c|c|c|c|c|c|}
\hline & Vehicle & $\begin{array}{c}\text { \# Tests } \\
\text { Consid- } \\
\text { ered }\end{array}$ & $\begin{array}{l}\text { \# Tests } \\
\text { that TWL } \\
\text { occurred }\end{array}$ & $\begin{array}{c}\text { DSI/SSF } \\
\text { predict } \\
\text { TWL }\end{array}$ & $\begin{array}{l}\text { DSIISSF } \\
\text { predict TWL } \\
\text { and TWL } \\
\text { occurred }\end{array}$ & $\begin{array}{l}\text { DSIISSF } \\
\text { predict TWL } \\
\text { and TWL did } \\
\text { not occur }\end{array}$ & $\begin{array}{c}\text { TWL occurred } \\
\text { and DSI/SSF } \\
\text { did not predic } \\
\text { TWL }\end{array}$ \\
\hline 1 & 1991 Chevrolet Cavalier & & 0 & & & & \\
\hline 2 & 1992 Ford F-150 & & 0 & & & & \\
\hline 3 & 1992 Honda Civic LX & & 0 & & & & \\
\hline 4 & 1993 Chevrolet Caprice Classic & & 0 & & & & \\
\hline 5 & 1993 Ford Aerostar & 59 & 13 & 3 & 2 & 1 & 11 \\
\hline 6 & 1994 Chevrolet C1500 & & 0 & & & & \\
\hline 7 & 1994 Dodge Caravan & & 0 & & & & \\
\hline 8 & 1994 Ford Taurus & & 0 & & & & \\
\hline 9 & 1995 Chevrolet Astro & 30 & 12 & 3 & 2 & 1 & 10 \\
\hline 10 & 1995 Chevrolet K1500 & & 0 & & & & \\
\hline 11 & 1995 Ford Windstar GL & & 0 & & & & \\
\hline 12 & 1995 Mitsubishi Montero & 63 & 34 & 0 & 0 & 0 & 34 \\
\hline 13 & 1996 Acura SLX & 89 & 42 & 8 & 4 & 4 & 38 \\
\hline 14 & 1997 Chevrolet Metro & & 0 & & & & \\
\hline 15 & 1997 Ford F-150 & & 0 & & & & \\
\hline 16 & 1997 Ford Ranger $4 \times 2$ & & 0 & & & & \\
\hline 17 & 1997 Ford Ranger $4 \times 4$ & 38 & 7 & 8 & 7 & 1 & 0 \\
\hline 18 & 1997 Jeep Cherokee Sport & & 0 & & & & \\
\hline 19 & 1998 Chevrolet Tracker & 47 & 8 & 7 & 4 & 3 & 4 \\
\hline 20 & 1998 Honda CR-V & 55 & 12 & 7 & 3 & 4 & 9 \\
\hline 21 & 1998 Plymouth Voyager & & 0 & & & & \\
\hline 22 & 2001 Chevrolet Blazer & 58 & 21 & 9 & 3 & 6 & 18 \\
\hline 23 & 2001 Ford Explorer Sport 4x2 & & 0 & & & & \\
\hline 24 & 2001 Ford Explorer XLS 4x4 & 61 & 8 & 1 & 0 & 1 & 8 \\
\hline 25 & 2001 Toyota 4Runner & 14 & 9 & 11 & 9 & 2 & 0 \\
\hline 26 & 2002 Chevrolet Corvette & & 0 & & & & \\
\hline & Total & 514 & 166 & 57 & 34 & 23 & 132 \\
\hline
\end{tabular}

Table 3.1: Analysis of DSI/SSF as TWL Predictors (Phase VI Data) 
Given that the metrics both under and overestimated TWL, the previously mentioned threshold multiplier would decrease the number of underestimates (desirable) and increase the number of overestimates (undesirable). Instead of introducing a threshold multiplier, the current metrics should be modified to better represent the roll dynamics of the vehicle.

\subsection{Modification of SSF and DSI}

In the previous rollover index study, the metrics (DSI/SSF) correctly predicted TWL or no TWL $69.8 \%$ of the 514 Phase VI tests considered. In order to increase the accuracy of the metrics, an improved model of the vehicle roll dynamics is needed. Two relatively easy modifications to implement are:

1. Account for the decrease in track width due to the lateral deflection of the tires. This would decrease the effective SSF, which already accounts for the lateral CG offset. This modification would require knowing the lateral tire stiffness (lateral spring rate).

2. Increase the assumed value that the unsprung roll inertia is $80 \%$ of the total roll inertia. This modification would increase the DSI value. For a more accurate model, this percentage should be calculated for each vehicle. However, this is not feasible_- but perhaps an average value could be determined from several vehicles, in particular an average value found for each type of vehicle (passenger car, SUV, van, and light truck).

In the current study, the assumption that the unsprung roll inertia is $80 \%$ of the total roll inertia was replaced by an approximation for the unsprung roll inertia based on the type 
of suspension (solid rear axle or independent), total roll inertia of the vehicle, loaded radius of the tire, front and rear track width, unsprung mass, sprung mass, and sprung mass CG. This estimate was reported in "Vehicle Dynamic Stability and Rollover" by Systems Technology Incorporated (STI, June 1992) [18].

Four cases were considered in representing the decrease in track width due to the lateral deflection of the tires. All four cases used the approximation for the unsprung roll inertia suggested by STI. Additional dynamic effects were also considered in CASE 2 and 4.

\section{CASE 1:}

Assume tire lateral deflection $($ in $)=$ Lateral Force $*(0.0002 \mathrm{ft} / \mathrm{lb}) *(12 \mathrm{in} / \mathrm{ft})$. The deflection coefficient $(0.0002 \mathrm{ft} / \mathrm{lb})$ is based on static lateral tire stiffness tests performed at S.E.A., Inc. in Columbus, Ohio. During tip up, all mass is on one side of the vehicle; therefore the lateral force on one tire is equal to half the vehicle mass times the lateral acceleration. The lateral deflection of the tire (inches) is:

$$
\text { tire_deflect_1 }=a b s\left(0.5 * M * a_{y} * 0.0002 * 12\right)
$$

\section{CASE 2:}

Another approximation of the lateral tire deflection coefficient is 1.0 to 3.0 inches per $\mathrm{g}$ of lateral acceleration, which is based on tire test data [14]. Given that the majority of vehicles in Phase VI that experienced TWL were SUVs and that SUV tires tend to have higher aspect ratios (i.e., more lateral tire deflection), the deflection coefficient was assumed to be $2.5 \mathrm{in} / \mathrm{g}$. Another effect that shortens the moment arm of the stabilizing moment is the sprung mass rolling to the outside of a turn. Based on typical vehicle geometry and roll gain, this reduction is about $1.0 \mathrm{in} / \mathrm{g}$ for passenger 
cars and utility vehicles [14]. The total reduction of the moment arm is therefore 3.5 $\mathrm{in} / \mathrm{g}$, which is equivalent to (inches):

$$
\text { tire_deflect_ } 2=\operatorname{abs}\left(3.5 * a_{y}\right)
$$

\section{CASE 3:}

Similar to CASE 2, but assume multiplier of 4.0 instead of $3.5 \mathrm{in} / \mathrm{g}$, so that:

$$
\text { tire_deflect_3 }=\operatorname{abs}\left(4.0 * a_{y}\right)
$$

\section{CASE 4:}

Estimate the combined lateral compliance rate of the tire and suspension based on STI's vehicle model [18]. The combined rate accounts for tire casing lateral compliance, wheel bending, suspension bending, suspension and sub-frame bushing deflection, etc. This rate is estimated to be twice the vertical spring rate of the tire. The vertical spring rates of the tires used in Phase VI testing are unknown; therefore an approximation given in the report by STI was used. To validate the approximation equation for vehicles that showed TWL in Phase VI (mainly light trucks and SUVs), the approximation equation was used to estimate the vertical deflection of a P265/75R15 tire mounted on a 1997 Ford Ranger STX 4x4. The equation estimated the deflection to be about a tenth of an inch. However, the vertical deflection of the tire and vehicle combination based on actual measurements was about one inch. The approximation, based solely on tire dimensions and inflation pressure, greatly overestimated the vertical spring rate of a test vehicle. The approximation formula attempts to represent all passenger vehicle tires, which given the complexity of a tire, is probably unreasonable based solely on tire dimensions and inflation pressure. 
Based on the inaccuracies of the tire vertical spring rate calculation, the combined lateral compliance rate approximation (CASE 4) was not used.

In addition to testing the validity of the previously mentioned dynamic rollover indexes, the ' $\mathrm{R}_{2}$ ' dynamic rollover index from "A Robust Active Suspension Controller with Rollover Prevention" [17] was considered. It is based on the change of energy of a simplified sprung mass body model and the assumption that "the suspension travel range will not have too much of an impact on the potential energy change at the critical situation when the kinetic energy is to be greater than the potential energy change." The index was calculated for many vehicle runs, in which it failed to represent rollover propensity, and therefore the index was not applied to all Phase VI tests.

The results of the three cases are shown in Table 3.2. The ten vehicles that experienced TWL are shown in the table, and those vehicles that did not experience TWL were omitted. The first column in 'CASE 1' gives the number of times that CASE 1 DSI/SSF predicted TWL. The second column in 'CASE 1' states the frequency that CASE 1 DSI/SSF predicted TWL (first ' $Y$ ') and TWL occurred (second ' $Y$ '). The third through fifth columns are similar in nature. The last column of 'CASE 1' states the percentage that CASE $1 \mathrm{DSI} / \mathrm{SSF}$ correctly predicted TWL (correct being $\mathrm{Y} / \mathrm{Y}$ or N/N). The columns in 'CASE 2' and 'CASE 3' follow the same convention as the columns in 'CASE 1.' 


\begin{tabular}{|c|c|c|c|c|c|c|c|c|c|c|c|c|c|c|c|c|c|c|c|c|c|}
\hline & & & & \multicolumn{6}{|c|}{ CASE 1} & \multicolumn{6}{|c|}{ CASE 2} & \multicolumn{6}{|c|}{ CASE 3} \\
\hline & $\begin{array}{l}\text { Predict TWL: Y/N } \\
\text { TWL Occurred: Y/N }\end{array}$ & $\begin{array}{c}\text { \# Tests } \\
\text { Consid- } \\
\text { ered }\end{array}$ & $\begin{array}{c}\# \text { Tests } \\
\text { that TWL } \\
\begin{array}{c}\text { occur- } \\
\text { red }\end{array} \\
\end{array}$ & $\begin{array}{c}\text { DSI/SSF } \\
1 \\
\text { predict } \\
\text { TWL } \\
\end{array}$ & $\begin{array}{l}Y \\
Y \\
\end{array}$ & $\begin{array}{l}\mathbf{Y} \\
\mathrm{N} \\
\end{array}$ & $\begin{array}{l}\mathbf{N} \\
\mathbf{Y} \\
\end{array}$ & $\begin{array}{l}\mathbf{N} \\
\mathbf{N} \\
\end{array}$ & $\begin{array}{l}\% \\
\text { cor- } \\
\text { rect }\end{array}$ & \begin{tabular}{|c} 
DSI/SSF \\
2 \\
predict \\
TWL \\
\end{tabular} & $\begin{array}{l}\mathbf{Y} \\
\mathbf{Y} \\
\end{array}$ & $\begin{array}{l}\mathbf{Y} \\
\mathrm{N} \\
\end{array}$ & $\begin{array}{l}\mathbf{N} \\
\mathrm{Y} \\
\end{array}$ & $\begin{array}{l}\mathbf{N} \\
\mathbf{N} \\
\end{array}$ & $\begin{array}{l}\% \\
\text { cor- } \\
\text { rect }\end{array}$ & \begin{tabular}{|c|} 
DSI/SSF \\
3 \\
predict \\
TWL \\
\end{tabular} & $\begin{array}{l}Y \\
Y \\
\end{array}$ & $\begin{array}{l}\mathbf{Y} \\
\mathbf{N} \\
\end{array}$ & $\begin{array}{l}\mathrm{N} \\
\mathrm{Y} \\
\end{array}$ & $\begin{array}{l}\mathbf{N} \\
\mathbf{N} \\
\end{array}$ & $\begin{array}{l}\% \\
\text { cor- } \\
\text { rect }\end{array}$ \\
\hline 5 & 1993 Ford Aerostar & 59 & 13 & 35 & 13 & 22 & 0 & 24 & 62.7 & 23 & 10 & 13 & 3 & 33 & 72.9 & 25 & 11 & 14 & 2 & 32 & 72.9 \\
\hline 9 & 1995 Chevrolet Astro & 30 & 12 & 24 & 12 & 12 & 0 & 6 & 60 & 19 & 12 & 7 & 0 & 11 & 76.7 & 20 & 12 & 8 & 0 & 10 & 73.3 \\
\hline 12 & 1995 Mitsubishi Montero & 63 & 34 & 29 & 21 & 8 & 13 & 21 & 66.7 & 9 & 9 & 0 & 25 & 29 & 60.3 & 11 & 10 & 1 & 24 & 28 & 60.3 \\
\hline 13 & 1996 Acura SLX & 89 & 42 & 86 & 40 & 46 & 2 & 1 & 46.1 & 62 & 32 & 30 & 10 & 17 & 55.1 & 68 & 34 & 34 & 8 & 13 & 52.8 \\
\hline 17 & 1997 Ford Ranger $4 x 4$ & 38 & 7 & 12 & 7 & 5 & 0 & 26 & 86.8 & 9 & 7 & 2 & 0 & 29 & 94.7 & 11 & 7 & 4 & 0 & 27 & 89.5 \\
\hline 19 & 1998 Chevrolet Tracker & 47 & 8 & 18 & 7 & 11 & 1 & 28 & 74.5 & 17 & 7 & 10 & 1 & 29 & 76.6 & 19 & 7 & 12 & 1 & 27 & 72.3 \\
\hline 20 & 1998 Honda CR-V & 55 & 12 & 24 & 12 & 12 & 0 & 31 & 78.2 & 20 & 12 & 8 & 0 & 35 & 85.5 & 24 & 12 & 12 & 0 & 31 & 78.2 \\
\hline 22 & 2001 Chevrolet Blazer & 58 & 21 & 40 & 20 & 20 & 1 & 17 & 63.8 & 31 & 20 & 11 & 1 & 26 & 79.3 & 33 & 20 & 13 & 1 & 24 & 75.9 \\
\hline 24 & $\begin{array}{l}2001 \text { Ford Explorer XLS } \\
4 \times 4\end{array}$ & 61 & 8 & 8 & 5 & 3 & 3 & 50 & 90.2 & 3 & 2 & 1 & 6 & 52 & 88.5 & 5 & 3 & 2 & 5 & 51 & 88.5 \\
\hline 25 & 2001 Toyota 4Runner & 14 & 9 & 14 & 9 & 5 & 0 & 0 & 64.3 & 12 & 9 & 3 & 0 & 2 & 78.6 & 13 & 9 & 4 & 0 & 1 & 71.4 \\
\hline & Total & 514 & 166 & 290 & 146 & 144 & 20 & 204 & 69.3 & 205 & 120 & 85 & 46 & 263 & 76.8 & 229 & 125 & 104 & 41 & 244 & 73.5 \\
\hline
\end{tabular}

Table 3.2: Analysis of the Modified DSI/SSF Metrics as TWL Predictors 
The accuracy of each case study considered, including the previous study of DSI/SSF ('Original'), is shown in Table 3.3.

\begin{tabular}{|c|c|c|}
\hline CASE & $\begin{array}{c}\text { \% Correct } \\
\text { (total tests) }\end{array}$ & $\begin{array}{c}\text { \% Correct (equal } \\
\text { vehicle weight) }\end{array}$ \\
\hline Original & 69.8 & 73 \\
\hline 1 & 68.1 & 69.3 \\
\hline 2 & 74.5 & 76.8 \\
\hline 3 & 71.8 & 73.5 \\
\hline
\end{tabular}

Table 3.3: Accuracy of Rollover Stability Indexes

where:

$\%$ Correct $($ total tests $)=($ total correct $) /($ total tests considered $)$

$\%$ Correct (equal vehicle weight $)=($ Sum of $\%$ correct for each vehicle $) /($ number of vehicles)

The "percentage correct using equal vehicle weighting" eliminates the effect that a particular vehicle with a proportionally large number of tests, either well or poorly represented by the index, dominates.

This study is a step in the right direction to better represent the roll dynamics of the vehicle. Unfortunately, the modified indexes show at most a $6.7 \%$ increase of accuracy (for CASE 2: $(74.5-69.8) / 69.8 * 100 \%=6.7 \%$ ).

As mentioned before, the indexes are limited by the assumptions used. Parameters such as lateral and vertical tire deflection (stiffness), sprung mass inertia, sprung mass CG height, etc. are based on calculations that use coefficients derived from a 
limited number of vehicle tests and thus may or may not represent accurately the current vehicle under consideration. This makes predicting TWL very difficult at the TWL threshold. The metrics, with their many assumptions, may well represent the rollover propensity of vehicles during no wheel lift and major wheel lift (more than two inches), but not at the TWL threshold (simultaneous front and rear lift of two inches).

More complex models are needed to fully represent the roll dynamics of the vehicle. For example, a two degree of freedom model (separate sprung and unsprung masses) would represent the sprung mass rolling to the outside of turn, thus shortening the moment arm of the stabilizing moment. However, the two degree of freedom model requires more vehicle parameters to be known, e.g., roll center, suspension kinematics, etc. The current model (CASE 2) accounts for the shift of the sprung mass by assuming a shift of 1.0 inch per lateral g (suggested in [14]). According to Hac [16], other factors that affect the roll dynamics include the change in half-track width due to suspension kinematics and compliance, gyroscopic forces due to wheel rotation (increase the moment needed for rollover), and vertical forces transmitted from the wheels through the suspension that lift the vehicle CG height. These factors along with others that change the lateral forces (by approximately the percentage ranges given) needed to induce rollover are listed below in descending order of effect [16]:

1. lateral displacement of vehicle center of gravity due to body roll; 5 to $12 \%$ decrease

2. reduction in half track width due to lateral compliance of the tires; 3 to $8 \%$ decrease

3. increase in the center of gravity height resulting from jacking forces; up to $5 \%$ decrease

4. increase in half track width as the result of suspension kinematics; up to 5\% increase 
5. gyroscopic forces due to wheel rotation; 1 to $1.5 \%$ increase

\subsection{Conclusions and Recommendations of the Rollover Stability Index Study}

If the sole purpose of DSI/SSF is to accurately predict when TWL will occur, then the investigation should be continued. However, a more complex model will require knowing more vehicle parameters. At some point, adding more required vehicle parameters (which will require more testing) will outweigh the cost of simply testing the vehicle on the track to determine if and when TWL will occur. Perhaps a more useful purpose of DSI/SSF is to predict vehicle instability (impending wheel lift but not necessarily TWL) for the purpose of on-board vehicle stability control. For example, if DSI/SSF approaches 0.8 , then engine power will be reduced, the appropriate brakes will be applied, suspension force actuators will act to reduce the roll angle, etc., such that the vehicle will go from an impending unstable condition to a stable one.

If it is desired to have DSI/SSF more accurately predict TWL, then the following recommendations should be considered:

1. Perform vehicle tests to determine the total reduction of the effective half track width (lateral tire deflection, suspension compliance, etc.) for each vehicle.

2. Incorporate the effect of roll angle into the index.

There have been several field tests where lateral acceleration was relatively low and yet the roll angle was relatively high and TWL occurred. An increase in roll angle is probably the result of increased vertical forces in the suspension. This scenario will be accompanied by an increase in CG height, which reduces the stabilizing moment. 
3. Further investigate the role of the suspension kinematics.

4. Modify DSI to account for the oscillatory energy transfer in the system (springs, shocks, tires, etc.), which occurs in vehicles that exhibit significant oscillatory roll response during severe maneuvers.

OR Keep DSI/SSF simple (as the original indexes) and note its limitations at the TWL threshold. 


\section{CHAPTER 4}

\section{EVALUATION OF THE FIELD TEST DATA}

\subsection{Introduction}

The fourth chapter provides an evaluation of the field tests in Phase VI. This includes a discussion of the accuracy and repeatability of the collected data, common vehicle asymmetries, vehicle understeer and roll gradients, and an analysis of the field test data.

As shown in Table 2.2, during Phase VI testing three vehicles exhibited significant response asymmetries with respect to the minimum maneuver entrance speed required to produce TWL. These vehicles are the 1996 Acura SLX ("SLX"), 1995 Mitsubishi Montero (“Montero"), and the 1993 Ford Aerostar (“Aerostar”).

\subsection{Accuracy and Repeatability of the Collected Data}

Before analyzing any experimental data, one must be certain that the experiments were executed properly and consistently and that the results are accurate and repeatable. As detailed in Chapter 2, the Phase VI testing protocol was based on a well developed and documented process. Phase VI field tests were carried out according to that protocol and all inputs (speed, handwheel angle, environmental conditions, etc.) were appropriately recorded. The objective of the Phase VI tests was to determine the 
minimum maneuver entrance speed at which TWL occurred. Therefore, in addition to measuring the wheel heights (TWL occurrence) onboard, the heights were also verified through video analysis. All other collected data was evaluated to ensure it makes physical sense. In other words, the collected data is accurate and repeatable (with the disclaimer given in Chapter 2 regarding test-to-test variability and TWL threshold vehicle response).

Ideally all tests should be performed according to the test protocol, and all commanded inputs should be executed accordingly. However, in real world testing, especially when dealing with a multibody nonlinear system such as a vehicle, slight differences in the inputs and the system itself over time may affect the system response. If these differences are consistent (e.g. handwheel rates achieved are 5\% higher than the commanded rate), then comparisons between tests can easily be made. However, if the differences are inconsistent (e.g. handwheel rates achieved vary from $95 \%$ to $105 \%$ of the commanded rate), then the differences in the inputs must be considered when comparing similar tests. Possible reasons for unintended differences in inputs/responses are detailed below:

\subsubsection{Commanded steering inputs (handwheel position and rate)}

A programmable steering machine (handwheel controller) was used to produce the commanded steering inputs (handwheel position and rate). Although steering torque was not measured, it is likely that differences in torque required to rotate the handwheel clockwise (CW) versus counter-clockwise (CCW) exist. If so, then the handwheel controller may not have performed $\mathrm{CW}$ and $\mathrm{CCW}$ commanded rotation rates equally. 
During Phase VI testing [1], J-Turn handwheel angle data were averaged for 1000 ms after the instant the commanded angle was first achieved. Road edge recovery handwheel angle data were averaged from $80 \mathrm{~ms}$ after completion of the initial steer to the initiation of the reversal to determine initial steer magnitudes. The reversal steer magnitudes were determined by averaging the handwheel data for $1000 \mathrm{~ms}$ after the instant the commanded angle of the reversal was first achieved. The commanded and actual handwheel angle (degrees) and rate (degrees/second) for the J-turn and road edge recovery maneuvers are given in Table 4.1. The percent differences are based on the actual range produced by the handwheel controller. For example, the commanded angle and rate for the J-turn maneuver (both steer directions) for the SLX was $384^{\circ}$ at $1000^{\circ} \mathrm{sec}$, respectively. The actual range (averaged data as previously described) was $384-386^{\circ}$ (negligible difference: “---") at 1114-1146\%/sec (2.8\% difference). The commanded range difference is negligible (even if left versus right steer were at the opposite extremes, i.e., $384^{\circ}$ to the left and $386^{\circ}$ to the right), however the $2.8 \%$ differences in handwheel rate may have an effect on the vehicle response, e.g. yaw rate and lateral acceleration.

Significant differences of handwheel angle and/or rates between left versus right steer maneuvers may contribute to vehicle asymmetric response. Cases to consider further are shaded in Table 4.1 and include the Montero's handwheel rate for the J-turn and the Aerostar's handwheel rate for the reversal steer in the road edge recovery maneuver. Note these differences may occur for the same direction of steer, in which case the different handwheel angle and/or rate has no effect on left versus right steer vehicle asymmetric response. For example, the actual rate range limits for the Montero 
in the J-turn maneuver (947 and $1030 \mathrm{deg} / \mathrm{sec}$ ) are both left steer maneuvers in the maximum occupancy load configuration. Vehicle response comparisons made in Section 4.6, "Analysis of Field Test Data," will consider differences in handwheel angle and rate.

\begin{tabular}{|c|c|c|c|c|c|}
\hline & & & SLX & Montero & Aerostar \\
\hline \multirow{6}{*}{\multicolumn{2}{|c|}{ J-turn }} & Commanded Angle & 384 & 336 & 451 \\
\hline & & Actual Angle Range & $384-386$ & $333-334$ & $456-458$ \\
\hline & & $\%$ Difference & --- & --- & --- \\
\hline & & Commanded Rate & 1000 & 1000 & 1000 \\
\hline & & Actual Rate Range & $1114-1146$ & $947-1030$ & $1116-1135$ \\
\hline & & $\%$ Difference & 2.8 & 8.4 & 1.7 \\
\hline \multirow{12}{*}{$\begin{array}{l}\text { Road Edge } \\
\text { Recovery }\end{array}$} & \multirow{6}{*}{$\begin{array}{l}\text { Initial } \\
\text { Steer }\end{array}$} & Commanded Angle & 312 & 273 & 366 \\
\hline & & Actual Angle Range & $310-312$ & $270-272$ & $376-377$ \\
\hline & & $\%$ Difference & --- & --- & --- \\
\hline & & Commanded Rate & 720 & 720 & 720 \\
\hline & & Actual Rate Range & $739-754$ & $712-733$ & $745-752$ \\
\hline & & $\%$ Difference & 2.0 & 2.9 & 0.9 \\
\hline & \multirow{6}{*}{$\begin{array}{l}\text { Reversal } \\
\text { Steer }\end{array}$} & Commanded Angle & 312 & 273 & 366 \\
\hline & & Actual Angle Range & $312-313$ & $270-273$ & $369-371$ \\
\hline & & $\%$ Difference & --- & --- & --- \\
\hline & & Commanded Rate & 720 & 720 & 720 \\
\hline & & Actual Rate Range & $707-721$ & $713-721$ & $590-722$ \\
\hline & & $\%$ Difference & 2.0 & 1.1 & 20.1 \\
\hline
\end{tabular}

Table 4.1: Handwheel Controller Angles (deg) and Rates (deg/sec) Based on Tables $6.1-6.4$ from [1]

\subsubsection{Initial handwheel angle prior to the maneuver}

Prior to a maneuver, the vehicle is driven in a "straight line" at a constant speed. Deviation from the straight line path is difficult for a driver to detect; therefore it is possible that the vehicle may have been driven with slight left or right steer prior to the maneuver. In addition, play/slop in the steering system may offset the actual handwheel angle achieved during the maneuver. 
Fortunately, data is recorded for two seconds just before the initial steer is executed, so any deviations from straight line driving and/or play in the handwheel can be detected. During those two seconds, the handwheel angle usually fluctuates between $\pm 2^{\circ}$. The entire data set is then zeroed based on the mean value of the handwheel angle during the last second prior to the initial steer input. Therefore, a vehicle would have to have considerable play (much greater than $4^{\circ}$ ) before it had a noticeable effect on vehicle response. For example, if the handwheel had $4^{\circ}$ of play, then the vehicle heading would be in a straight line when the handwheel angle was between $\pm 2^{\circ}$. If during both left and right steer J-turn maneuvers, the handwheel angle was $-2^{\circ}$ prior to the maneuver with a commanded maximum absolute handwheel angle of $360^{\circ}$, then the effective handwheel angle would be $-362^{\circ}$ for a left steer maneuver and $358^{\circ}$ for a right steer maneuver.

In addition, the handwheel angle, yaw rate, and lateral acceleration were analyzed to ensure the vehicle was driven in a straight line and the instrumentation zero point was the same as the true vehicle zero point.

\subsubsection{Environmental effects}

Although all of the Phase VI tests for a given vehicle were performed within a short time span (less than 16 days), day-to-day environmental fluctuations may affect the vehicle response. The time period of the tests as well as temperature ranges are as follows (all tests performed on dry pavement):

SLX time period: June $7-13,2002\left(72\right.$ to $92^{\circ}=20^{\circ} \mathrm{F}$ difference $)$

Montero: June 20 - July 3, 2002 ( 74 to $90^{\circ}=16^{\circ} \mathrm{F}$ difference)

Aerostar: September $16-$ October $1,2002\left(55\right.$ to $84^{\circ}=29^{\circ} \mathrm{F}$ difference $)$ 
A recent study of the effect of temperature on vehicle response in the J-turn and road edge recovery maneuvers was performed at VRTC [19]. A 2001 Toyota 4Runner 4WD (with and without yaw stability control enabled) and a 2001 Chevrolet Blazer 2WD were tested on the same test track during cold, moderate, and hot ambient temperatures. The temperature averages were $30^{\circ} \mathrm{F}$ (cold), $76^{\circ} \mathrm{F}$ (moderate), and $90^{\circ} \mathrm{F}$ (hot). The vehicles were tested according to the Phase VI protocol (e.g. increase maneuver entrance speed by $5 \mathrm{mph}$ until TWL occurs or maximum test speed is achieved). Out of the eighteen tests performed (essentially three vehicles, two maneuvers, three temperature ranges), the only temperature effect observed was that the Blazer tipped up in the J-Turn in cold weather $\left(29^{\circ} \mathrm{F}\right)$ but did not in the moderate $\left(83^{\circ} \mathrm{F}\right)$ and hot weather tests $\left(86^{\circ} \mathrm{F}\right)$. In all of the other tests, the vehicle/maneuver combination produced TWL at about the same speed in either all temperature ranges or none. Therefore, given that the maximum temperature range in the Phase VI testing was relatively small $\left(29^{\circ} \mathrm{F}\right.$ for the Aerostar), the effect of temperature on vehicle response is assumed to be negligible.

The maximum allowed wind speed was $15 \mathrm{mph}$. There is the possibility that a sudden side gust of wind may affect the vehicle response. However, the tests are repeated to isolate the TWL threshold speed, so one particular test may be affected, but that group of tests performed at the TWL threshold speed are still valid.

\subsubsection{Vehicle Dynamic Area (VDA) test surface}

All Phase VI tests were performed on the VDA. It is an 1800 by 1200 foot, flat, paved surface with a one percent longitudinal grade for drainage. It was constructed to be a smooth, homogeneous surface, and it is constantly monitored (visually and SN 
testing). The coefficient of friction (Skid Number-SN) was measured twice a month (weather permitting) and varied very little from month to month. The range of peak SN was $0.95-0.99$ and sliding SN was $0.83-0.88$ from May to October of 2003 [1].

Given the consistency of the test surface, it is unlikely that any road irregularities affected the vehicle response. The longitudinal grade may have a slight affect on vehicle response. All J-turn and road edge recovery maneuvers are performed directly climbing the grade (i.e., not across the grade), so any possible effects due to the grade are minimized.

\subsubsection{Tire wear}

Based on numerous tests performed at VRTC, it has been shown that tire wear during testing tends to increase the maximum lateral force capabilities of the tires and thus increases the maximum lateral acceleration of the vehicle and the vehicle's likelihood to tip up at a given speed. To minimize these effects, the tires were changed after each test series, e.g., one test series consists of both left and right J-turn tests in the nominal load condition. However, the tire change interval may be too long, especially since different tires wear at different rates and in different ways. In addition, the speed at which the tests are performed has a pronounced effect on the severity of the wear (the greater the maneuver entrance speed, the greater the tire wear). The following three studies investigate the number of vehicle tests needed for tire wear to have a noticeable effect on vehicle performance:

To test the effect of tire wear, VRTC performed 22 left-right road edge recovery tests with a 2001 Ford Explorer 4x4 (maximum occupancy) at the same entrance speed of 
$40 \mathrm{mph}$ (range of $39.4-40.8 \mathrm{mph}$ ) and using the same steering inputs. The first 18 tests did not produce TWL (some lift, but less than two inches of front and rear wheel lift), but 3 of the last 4 tests did [not published].

In “Assessing the Feasibility of Using ASTM E1136 Standard Reference Test Tires During Road Edge Recovery Maneuvers” [20], 25 left-right road edge recovery tests at $42 \mathrm{mph}$ entrance speed (range of $40.7-43.2 \mathrm{mph}$ ) were performed with a 1997 Ford Ranger 4x2 (ASTM E1136 Tires, 284lbs of roof ballast, $288^{\circ}$ left-right steering). Each of the 25 tests produced at least 3" of front wheel lift after initiation of the steering reversal, and 22 of these tests produced front wheel lift of at least 4". The eighth test produced minor TWL (front wheel lift of 3" and rear wheel lift of 0.5 "). The first test to produce major TWL (at least 2" for the front and rear wheel) was the eleventh test. After the eleventh test, major TWL was observed during 8 of the remaining 14 tests.

According to the study "Tire Shoulder Wear in Repetitive Rollover Testing” by Automotive Testing, Inc. [21], "the effect of tire shoulder wear on a vehicle's lateral acceleration and on the most-loaded tire's lateral deformation in 19 identical severe maneuvers was found to be negligible, at least for the vehicle/tire (2001 Chevrolet Tracker with OEM Uniroyal Tiger Paw AWP tires) measured.” The severe maneuver was similar to the road edge recovery maneuver with $180^{\circ}$ left/right steer at $50 \mathrm{mph}$.

Based on the previously mentioned studies and the Phase VI data, tire wear has an effect on vehicle response, but the effect is not noticeable if the number of tests performed on a set of tires is kept small. For some vehicle/tire/test combinations, "small" may be 5 tests, while for other combinations, "small" might be 18 tests (as with the 2001 Ford Explorer 4x4 described earlier). 
4.2.6 Vehicle age and wear: worn or damaged suspension components

Before testing any vehicle, especially used vehicles, the vehicle must be inspected to ensure performance similar to manufacturer design. As mentioned in Chapter 2, the suspension components of the used vehicles were refurbished along with any other components that did not meet the manufacturers standards. Also, a four-wheel alignment was performed before testing. At a minimum, the following items were replaced, regardless of their condition:

1. Front and rear shock absorbers / struts

2. Front and rear springs

3. Front and rear shock absorber / strut bump stops

4. Front strut bearings and any related bushings

5. Rear shock bushings

6. Front and rear swaybar bushings

\subsubsection{Test-to-test variability}

In addition to the variability just mentioned (tire wear, commanded handwheel angle and rate, environment, test surface, etc.), other test-to-test variability may occur. This is especially likely when vehicles are being evaluated at their TWL threshold speeds (the maneuver entry speed for which TWL may or may not occur). When operating at this threshold, the propensity of TWL is very high, but not guaranteed.

Consider Figure 4.1, which compares six, left steer J-turn tests performed consecutively on the same day by the SLX, with speeds ranging from 38.1 to $40.4 \mathrm{mph}$. 
At these threshold speeds, there were significant fluctuations of the lateral acceleration and roll angle, rate, and acceleration, even though all inputs were similar (fairly new tires, entrance speed, steer angle and rates, and environmental conditions). 

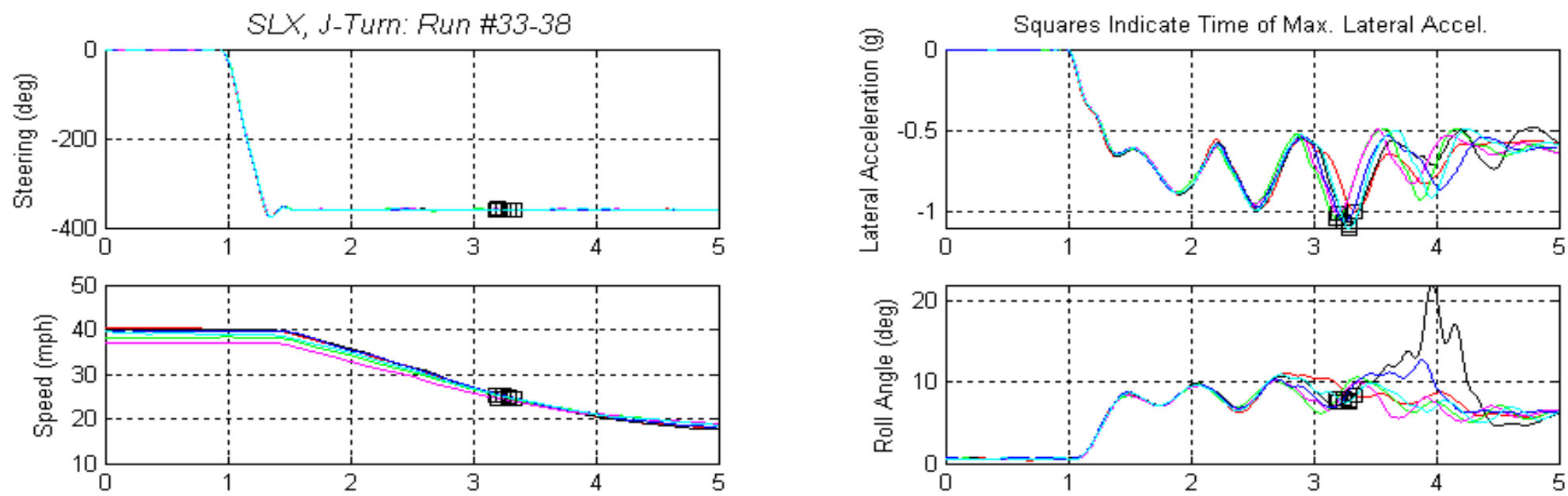

$+\infty$
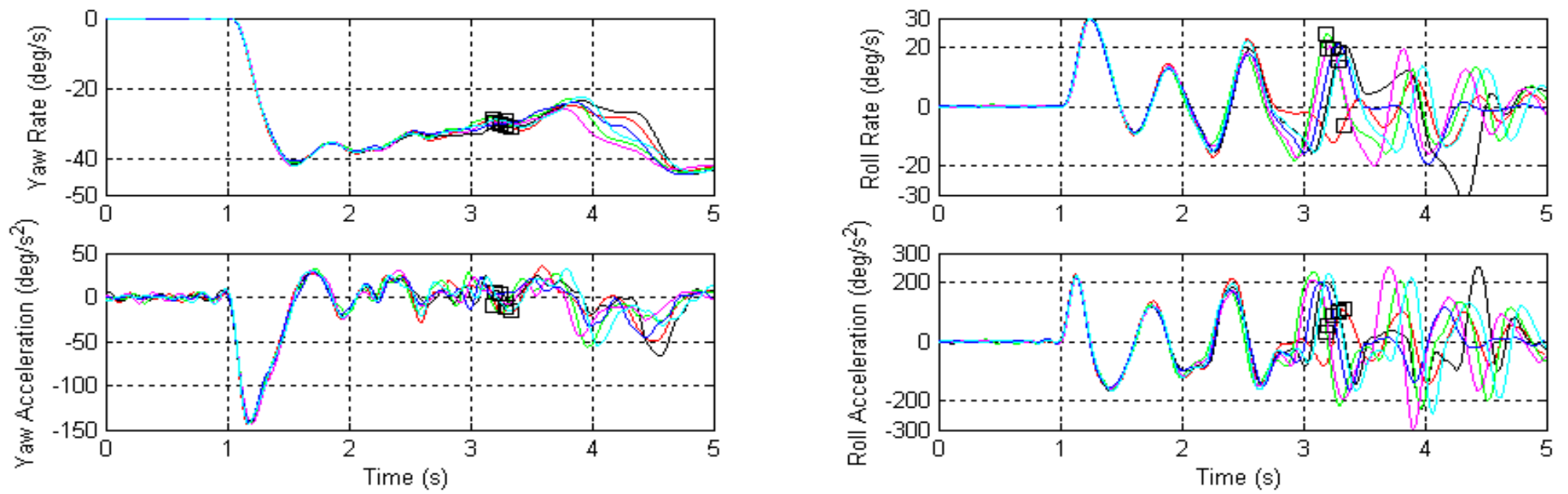

Figure 4.1: Comparison of SLX Response at $\approx 39 \mathrm{mph}$, Nominal Load, Left Steer J-turn 


\begin{tabular}{|c|c|c|}
\hline \multicolumn{1}{|l|}{ Run \# } & Entry Speed (mph) & Color \\
\hline 33 & 40.4 & red \\
\hline $34--T W L$ & 39.9 & black \\
\hline 35 & 38.1 & green \\
\hline 36 & 37.0 & magenta \\
\hline $37--T W L$ & 39.5 & blue \\
\hline 38 & 39.1 & cyan \\
\hline
\end{tabular}

Legend for Figure 4.1

(If viewed as a black \& white document, the important note to make is the roll angle plot that is singled out in Figure 4.2.)

The differences in the roll angle are also shown in Figure 4.2. Note that test 34 and 37 produced a maximum roll angle of $22^{\circ}$ and $13^{\circ}$, respectively, while the other four tests only reached $10-11^{\circ}$ of roll. The effect of tire wear was not apparent here.

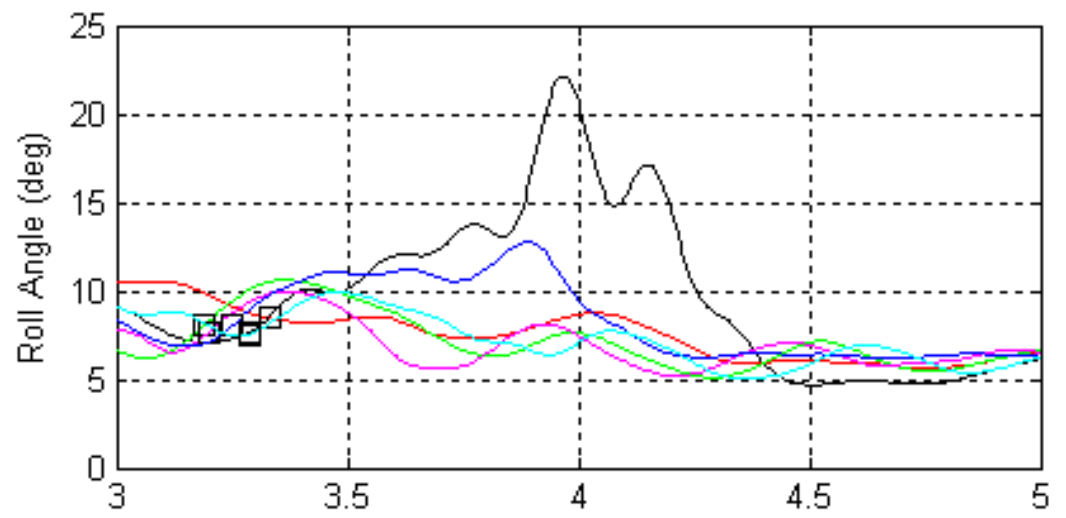

Figure 4.2: Differences in Roll Angle

According to a 2001 NHTSA report on rollover resistance [19, Notice 2], the authors "estimate that the speed at tip-up [for the J-turn and fishhook (road edge recovery) maneuvers] is repeatable within $2 \mathrm{mph}$ on the same surface." Thus, the 
variation in vehicle response shown in Figure 4.1 is not uncommon, even within the small range of vehicle speed (38.1 to $40.4 \mathrm{mph}$ ).

\subsection{Common Vehicle Asymmetries}

The tire lateral force as a function of load for a pair of wheels on an axle is maximized when the wheels are loaded equally [22]. This is because the tire cornering coefficient (cornering stiffness divided by load) is usually largest at light loads, diminishing continuously as the load reaches its rated value [13]. However, the wheels are usually not loaded equally. Reasons for this unequal load (left versus right wheel on a given axle) include static lateral CG offset, weight jacking (spring preload), and antiroll bar preload. Other common asymmetries include tire and tire pressure differences.

Vehicles commonly have a static lateral CG offset toward the driver side,

primarily due to the steering system and other vehicle controls located on the driver side. The lateral CG offsets for the three vehicles under consideration are (negative indicates offset toward the driver side):

$$
\begin{aligned}
& \text { SLX: -0.46" Nominal, -0.55" Max Occupancy } \\
& \text { Montero: }-1.13 " \text { Nominal, -1.08" Max Occupancy } \\
& \text { Aerostar: -0.90" Nominal, -1.04" Max Occupancy }
\end{aligned}
$$

Due to the offsets, the loads on the tires are more balanced in a left steer maneuver (the lateral CG offset compensates for the normal lateral weight transfer in a turn). Consequently, the vehicle should be able to achieve more lateral acceleration steering left. 
Weight jacking and roll bar preload affect wheel load distribution but do not affect the CG position. They result in a weight increase on one diagonal (e.g., the right front and left rear) and a corresponding decrease on the opposite diagonal. As such, they change the maximum side force distribution front to rear (i.e., affect over/understeer) [22].

It is common for race cars to have different tires (stagger) and different tire pressures with respect to the left and right side of the vehicle. Although passenger vehicles have seemingly identical tires and tire pressures on the left and right side of the vehicle, the response of "identical" tires is rarely identical. For the purposes of this research, "identical" tires are assumed to perform identically, unless tested otherwise, e.g., vertical spring rates tested at S.E.A., Inc. presented in Chapter 5.

\subsection{Understeer and Roll Gradients}

The under/oversteer gradient is a measure of the vehicle's front and rear lateral force response to the handwheel steer angle. An understeer (positive gradient) vehicle loses front wheel traction before the rear wheels, and thus plows out at high lateral accelerations (the front tires become saturated so that an increase in the handwheel angle does not correspond to an increase in lateral acceleration). An oversteer (negative gradient) vehicle loses rear wheel traction before the front wheels, and thus loses directional stability at high lateral accelerations (the vehicle will spin out unless the handwheel angle is reduced). 
The roll gradient (gain) is defined as the degrees of body roll per lateral $\mathrm{g}$ of acceleration. Large differences between the left and right steer roll gradients are an indication of suspension irregularities.

Both the understeer gradient (deg of wheel steer/g) and the roll gradient (deg of body roll/g) were determined from the slowly increasing steer maneuver. The gradient calculations and the maneuver were based on the "Constant Speed, Variable Steer" test defined in SAE J266 [10]. As described in Chapter 2, the maneuver was performed at 50 mph in both the left and right steer directions. At time zero, the handwheel angle was linearly increased from $0^{\circ}$ to $270^{\circ}$ at a rate of $13.5^{\circ}$ /second and then held constant at $270^{\circ}$ for two seconds. The gradients under all test conditions (steer direction and load condition) are given in Table 4.2. Note that each gradient value shown in Table 4.2 is the average gradient based on at least three tests. The understeer and roll gradients of a vehicle refer to the linear range of vehicle response (handwheel angle versus lateral acceleration). As such, the gradients were based on the data from 0.1 to $0.4 \mathrm{~g}$ 's of lateral acceleration. Also included in Table 4.2 is the maximum consistent lateral acceleration obtained during the maneuver, i.e., when the vehicle begins to plow out. These differences in maximum lateral acceleration are common. In a recent comparison test by Automobile Magazine of four-door passenger cars under $\$ 30,000$, nine of the twelve vehicles tested achieved more lateral acceleration cornering left, and the remaining three had equal limits [24]. Three of the vehicles had 0.5-0.6 g's more cornering left.

A plot of the handwheel angle versus lateral acceleration for each test condition is given in Figures $4.3-4.5$. Figure 4.3 highlights the linear range used to determine the understeer gradient as well as the corresponding value of the understeer gradient, $\mathrm{K}$. 
Since only small differences in the roll gradient between left and right steer directions existed, only one plot of body roll angle versus lateral acceleration is given, Figure 4.6.

Although it is worthwhile to note the response asymmetries in this linear response range, those asymmetries may not be reflected at higher levels of lateral acceleration, e.g. during severe driving maneuvers. Furthermore, all three vehicles, especially the Montero, have lower understeer gradients steering left versus right. This indicates that for a given handwheel angle input, those vehicles will generate more lateral acceleration steering left. Based on that and the rollover models presented in Chapter 2 (where lateral acceleration was a primary effect), it would seem reasonable that both vehicles would be more likely to tip up in a left steer maneuver. According to Table 2.1 however, the SLX tips up at a lower speed in a left steer J-turn maneuver as expected, but the Montero tips up at a lower speed in a right steer J-turn maneuver.

\begin{tabular}{|c|c|c|c|c|c|c|c|}
\hline \multirow{2}{*}{} & \multicolumn{2}{|c|}{ SLX } & \multicolumn{2}{c|}{ Montero } & \multicolumn{2}{c|}{ Aerostar } \\
\cline { 2 - 8 } & $\begin{array}{c}\text { Steer } \\
\text { Direction }\end{array}$ & $\begin{array}{c}\text { Nominal } \\
\text { Load }\end{array}$ & $\begin{array}{c}\text { Maximum } \\
\text { Occupancy }\end{array}$ & $\begin{array}{c}\text { Nominal } \\
\text { Load }\end{array}$ & $\begin{array}{c}\text { Maximum } \\
\text { Occupancy }\end{array}$ & $\begin{array}{c}\text { Nominal } \\
\text { Load }\end{array}$ & $\begin{array}{c}\text { Maximum } \\
\text { Occupancy }\end{array}$ \\
\hline \multirow{2}{*}{$\begin{array}{c}\text { Understeer } \\
\text { Gradient } \\
\text { (deg/g) }\end{array}$} & Left & 3.0 & 3.6 & 2.8 & 2.5 & 5.9 & 6.0 \\
\cline { 2 - 8 } & Right & 3.7 & 3.7 & 4.6 & 4.0 & 7.2 & 6.7 \\
\cline { 2 - 8 } & $\%$ difference & 20.9 & 2.7 & 48.6 & 46.2 & 19.8 & 11.0 \\
\hline \multirow{2}{*}{$\begin{array}{c}\text { Roll } \\
\text { Gradient } \\
\text { (deg/g) }\end{array}$} & Left & 8.4 & 10.0 & 7.9 & 9.1 & 9.0 & 9.8 \\
\cline { 2 - 8 } & Right & 9.2 & 10.1 & 7.8 & 9.5 & 10.4 & 10.4 \\
\hline \multirow{2}{*}{$\begin{array}{c}\text { Maximum } \\
\text { Lateral } \\
\text { Acceleration } \\
\text { (g) }\end{array}$} & Lefference & 9.1 & 1.0 & 1.3 & 4.3 & 14.4 & 5.9 \\
\cline { 2 - 8 } & Right & 0.74 & 0.70 & 0.71 & 0.65 & 0.63 & 0.65 \\
\hline
\end{tabular}

Table 4.2: Understeer Gradient, Roll Gradient, and Maximum Lateral Acceleration; Slowly Increasing Steer Maneuver 
In the report, "Analysis of a Request for a Defect Investigation Submitted by Bridgestone/Firestone, Inc.” [25], Ford evaluated the variability in determining a vehicle's understeer gradient. Based on the Ford study and other sources of variability, the "ODI [Office of Defects Investigation] believes that unless differences of at least $1 \% \mathrm{~g}$ are found when comparing the measured understeer gradients of different vehicle models, one cannot confidently state that the vehicles truly have different understeer gradients." Due to test variability involved in determining the understeer gradient, only those differences greater that $1 \% \mathrm{~g}$ are considered significant and are consequently highlighted in Table 4.2. 

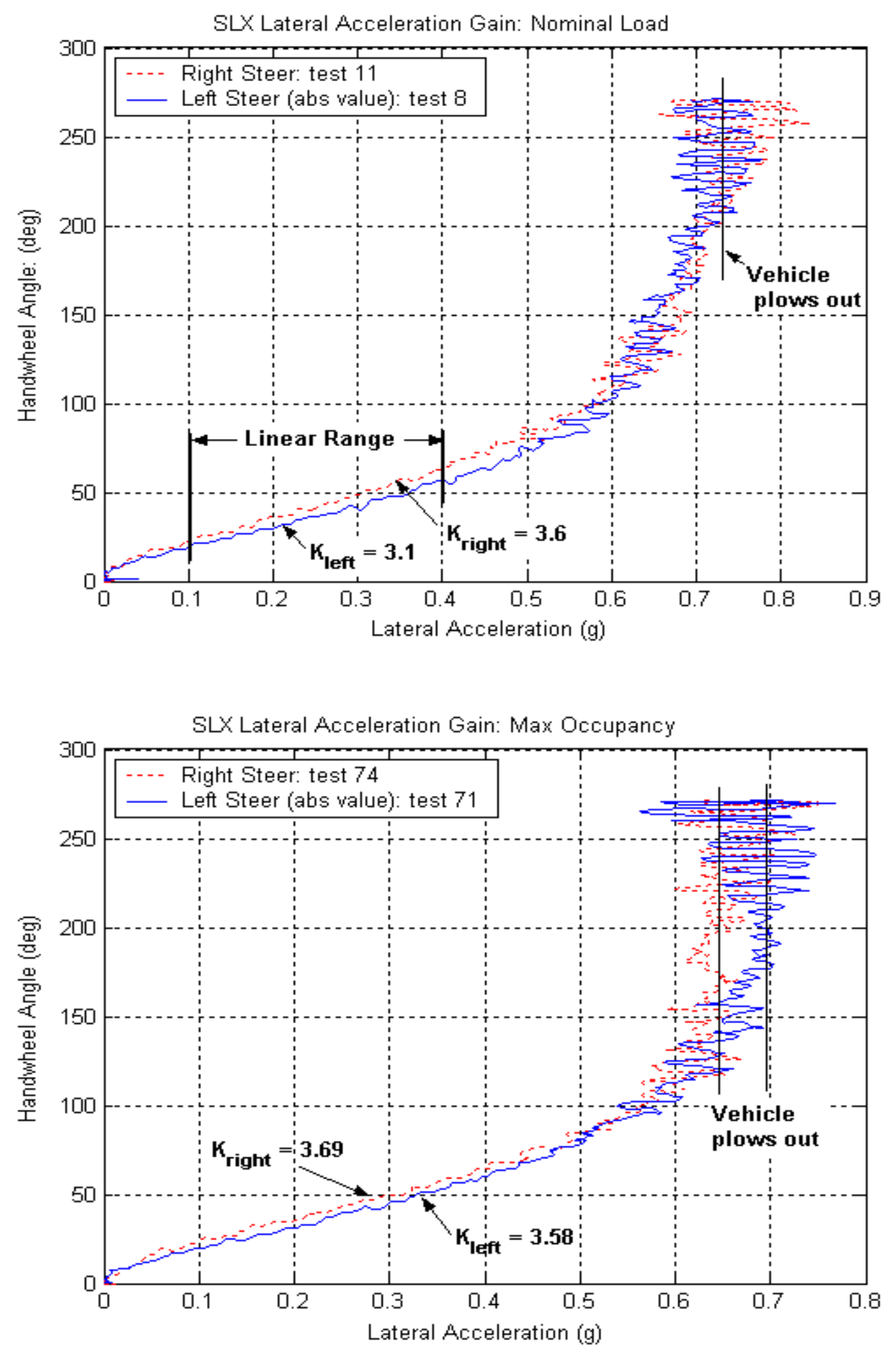

Figure 4.3: SLX, Slowly Increasing Steer Maneuver 

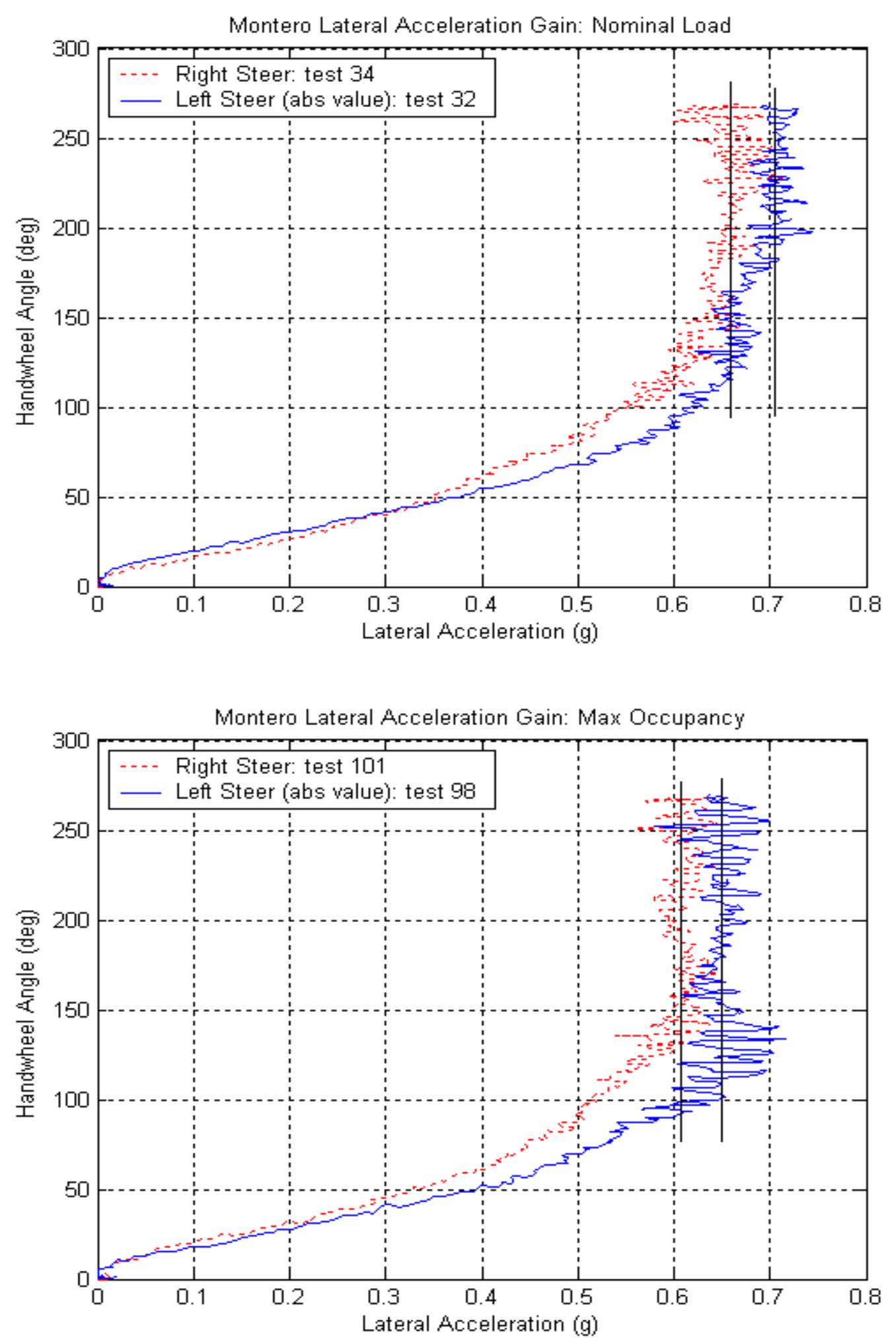

Figure 4.4: Montero, Slowly Increasing Steer Maneuver 

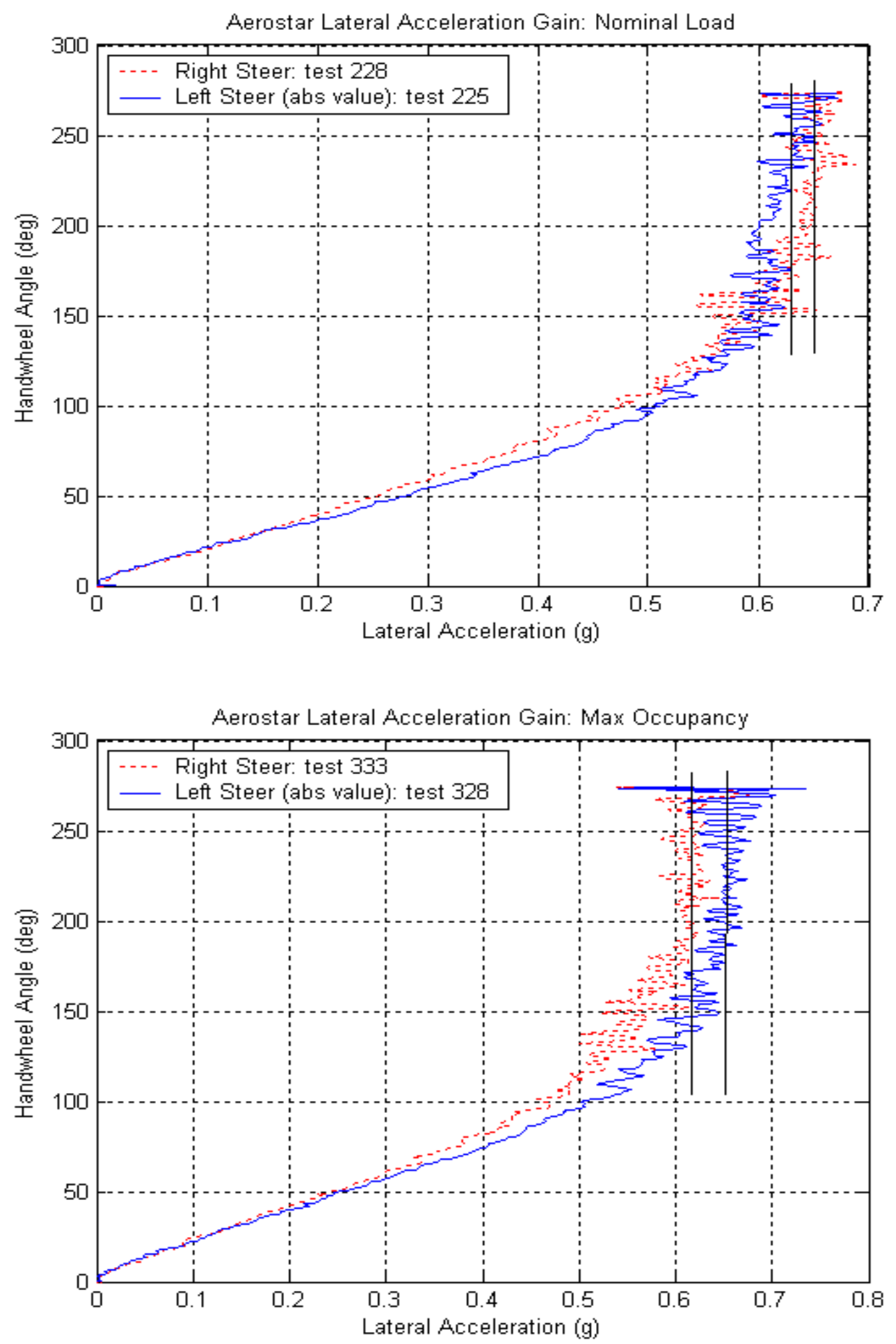

Figure 4.5: Aerostar, Slowly Increasing Steer Maneuver 


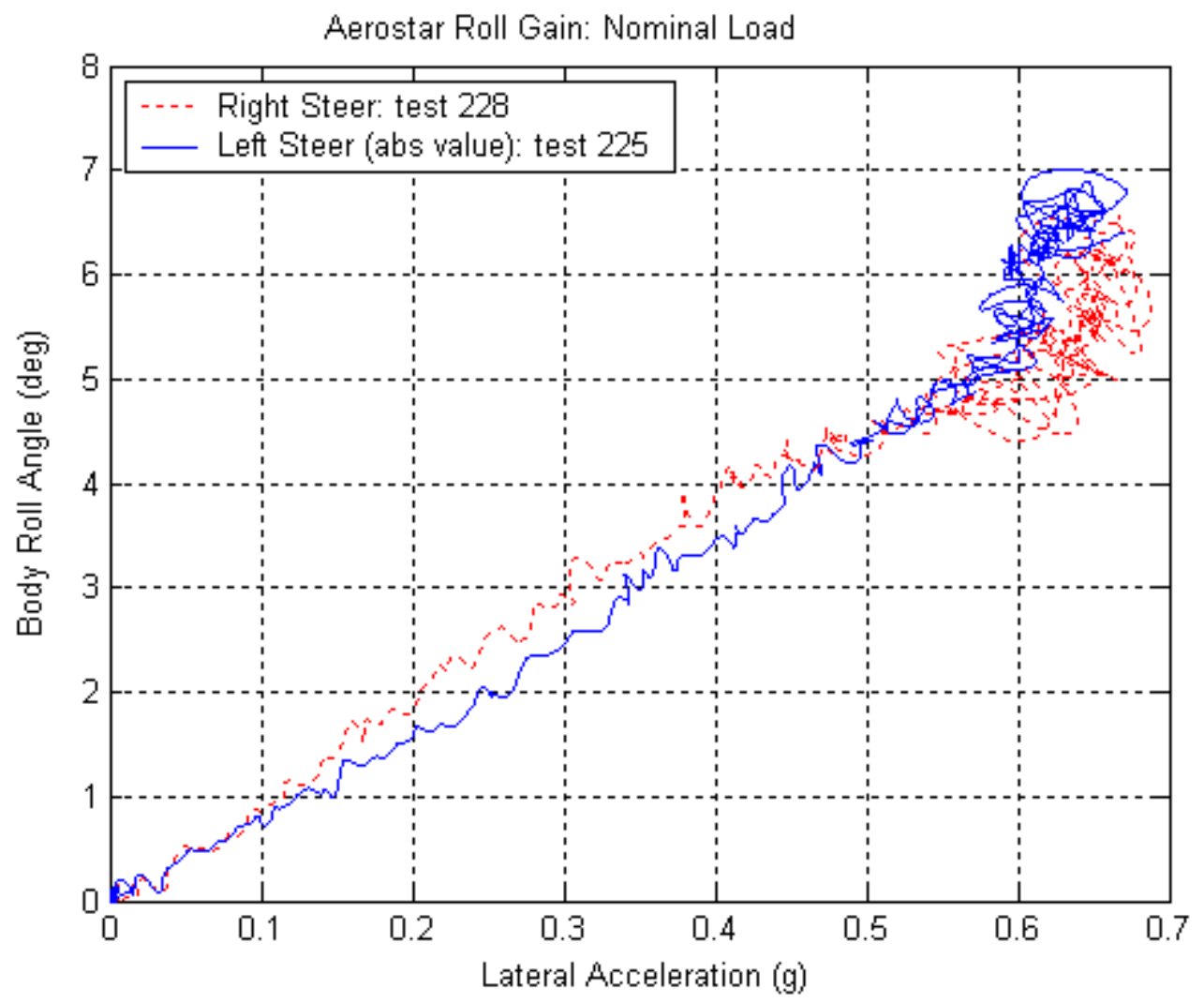

Figure 4.6: Aerostar Roll Gain, Slowly Increasing Steer Maneuver 


\subsection{Analysis of Field Test Data}

A comparison between both steering directions for the J-turn maneuver is shown in Figures 4.7 - 4.10 (SLX), 4.13 - 4.15 (Montero), and 4.18 - 4.20 (Aerostar). The comparison covers both load conditions and presents the vehicle response at the minimum maneuver entrance speed for which TWL was produced and at a common maneuver entrance speed. A similar comparison for the road edge recovery maneuver is shown in Figures 4.11 - 4.12 (SLX), 4.16 - 4.17 (Montero), and 4.21 - 4.22 (Aerostar). Since the differences in the minimum maneuver entrance speeds required to produce TWL are not as significant for the road edge recovery relative to the J-turn, only one load condition (that condition that produced more asymmetric response) is shown for the road edge recovery maneuver.

Neither the Montero nor the Aerostar experienced TWL in the J-turn nominal load condition test, therefore only the common maneuver entrance speed results are given. All three vehicles experienced TWL in the J-turn maximum occupancy test in only one steering direction (either left or right, not both); therefore the non-TWL steer direction entrance speed considered was $60 \mathrm{mph}$ (maximum test speed). For ease of comparison, all values are shown as absolute (positive). 

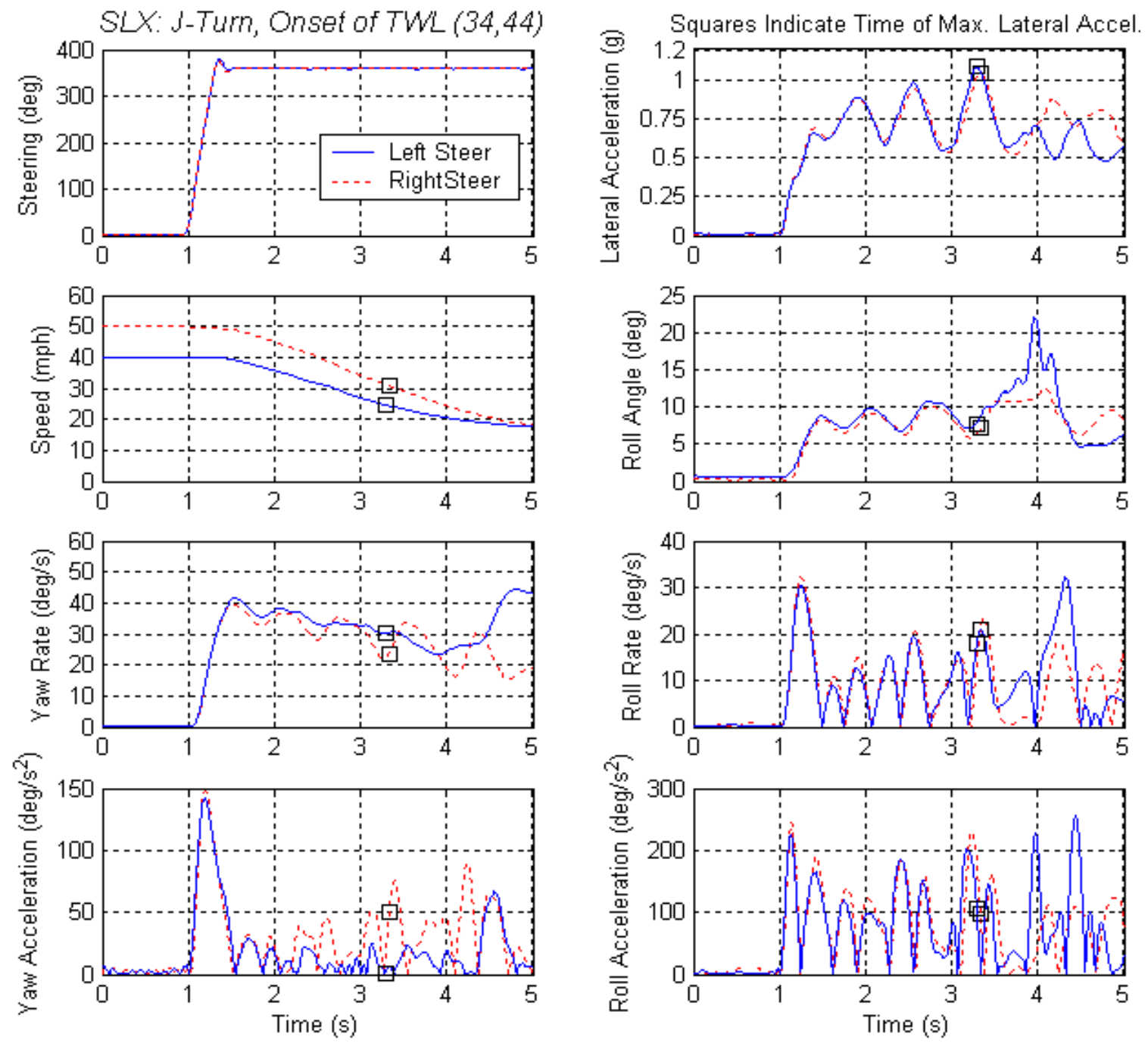

Figure 4.7: SLX, Nominal Load, J-turn at Onset of TWL Left Steer at $39.9 \mathrm{mph}$, Right Steer at $50.2 \mathrm{mph}$ 

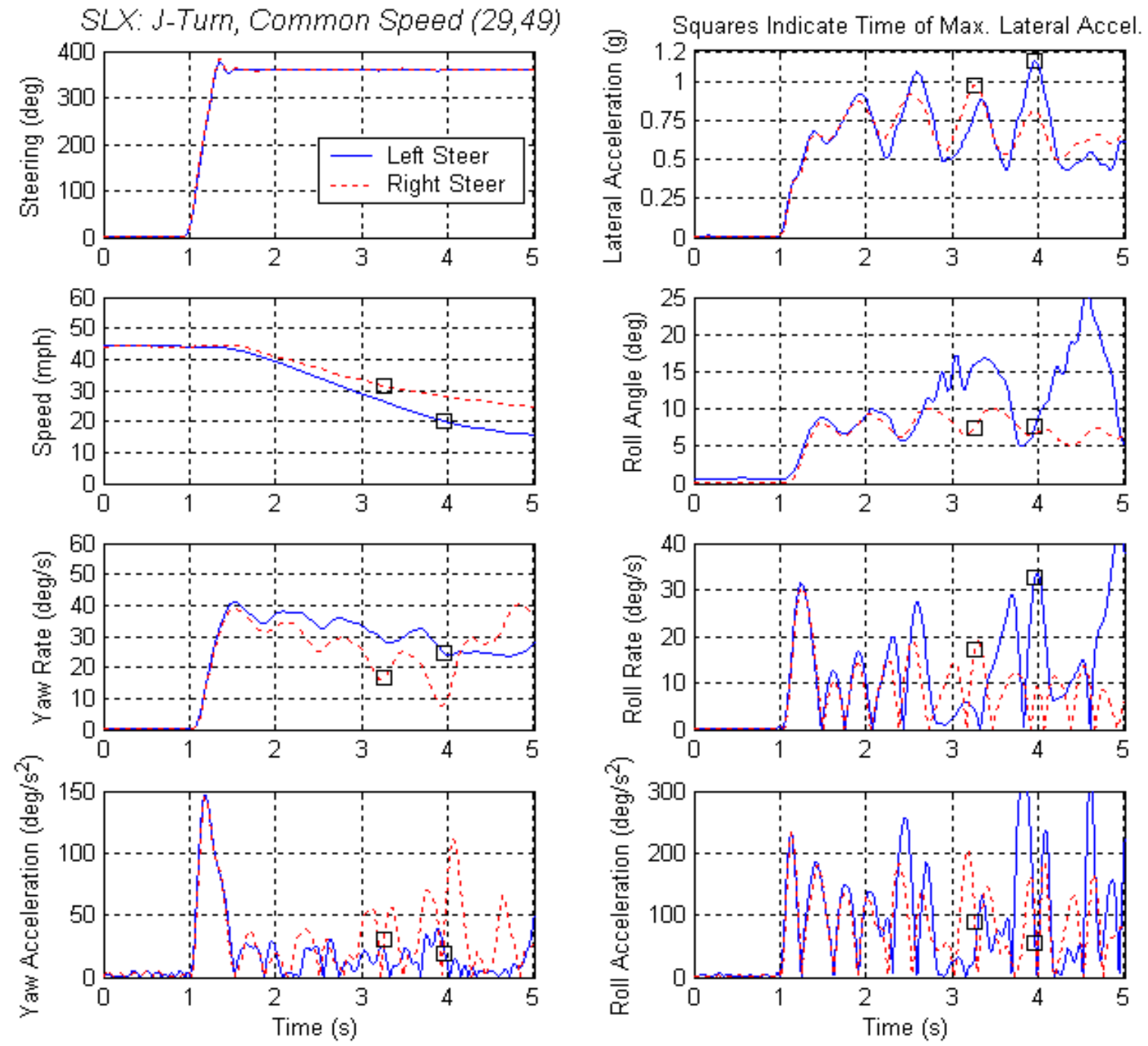

Figure 4.8: SLX, Nominal Load, J-turn at Common Entrance Speed Left Steer at $44.5 \mathrm{mph}$, Right Steer at $44.2 \mathrm{mph}$ 

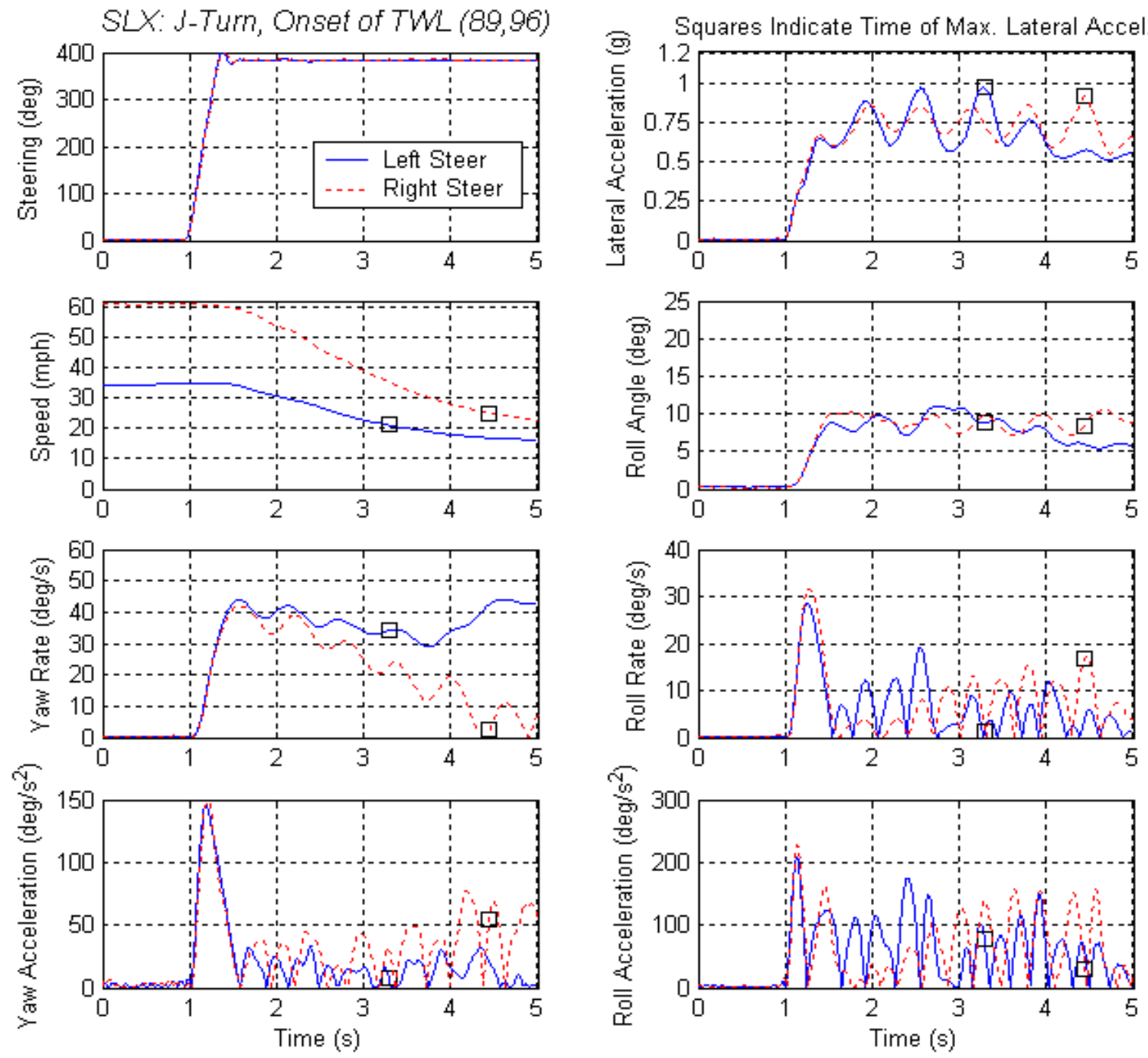

Figure 4.9: SLX, Maximum Occupancy, J-turn at Onset of TWL Left Steer at $34.6 \mathrm{mph}$, Right Steer at $61.1 \mathrm{mph} *$

*Note TWL did not occur, thus the maximum speed was used. 

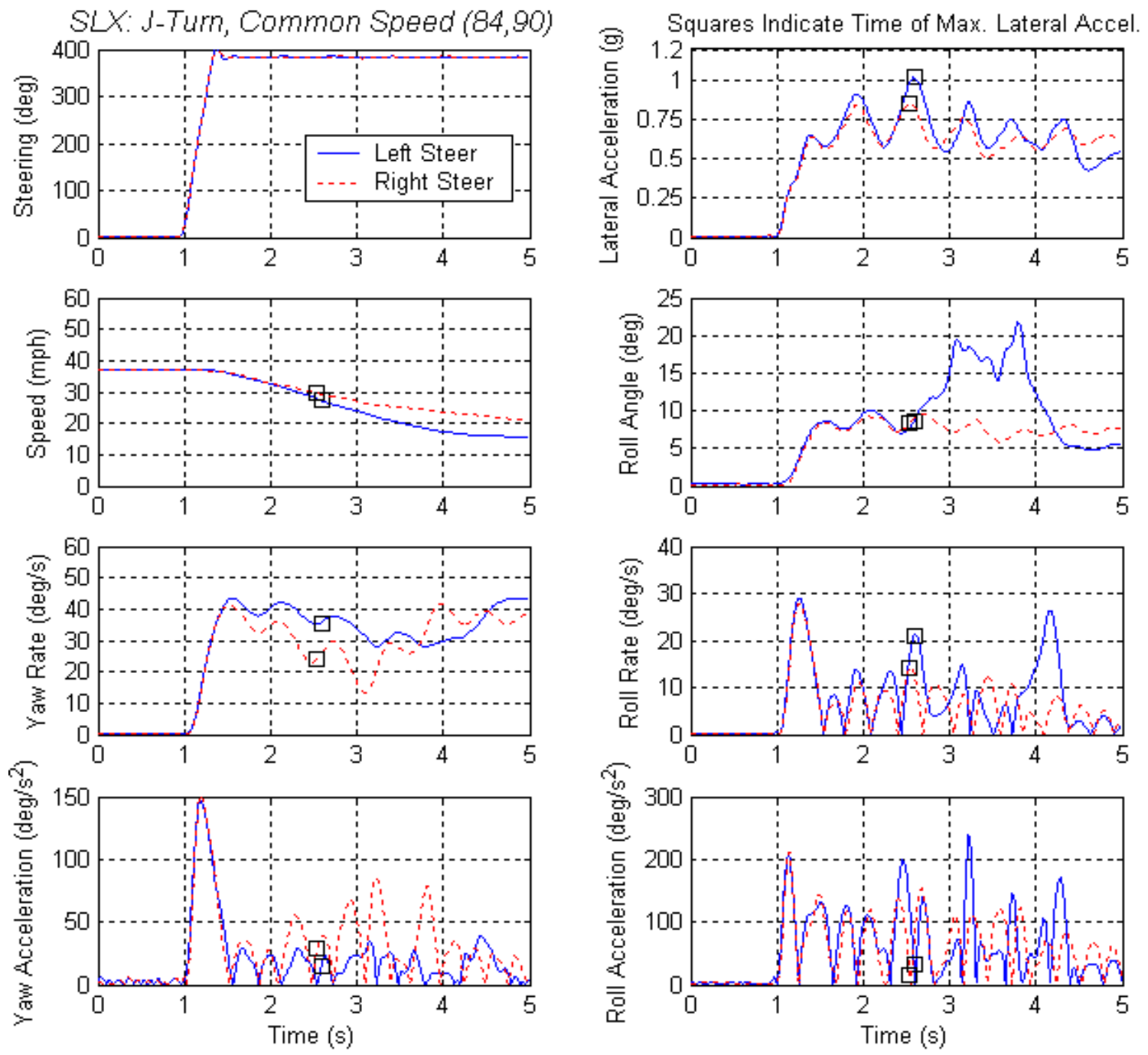

Figure 4.10: SLX, Maximum Occupancy, J-turn at Common Entrance Speed Left Steer at $36.9 \mathrm{mph}$, Right Steer at $37.0 \mathrm{mph}$ 

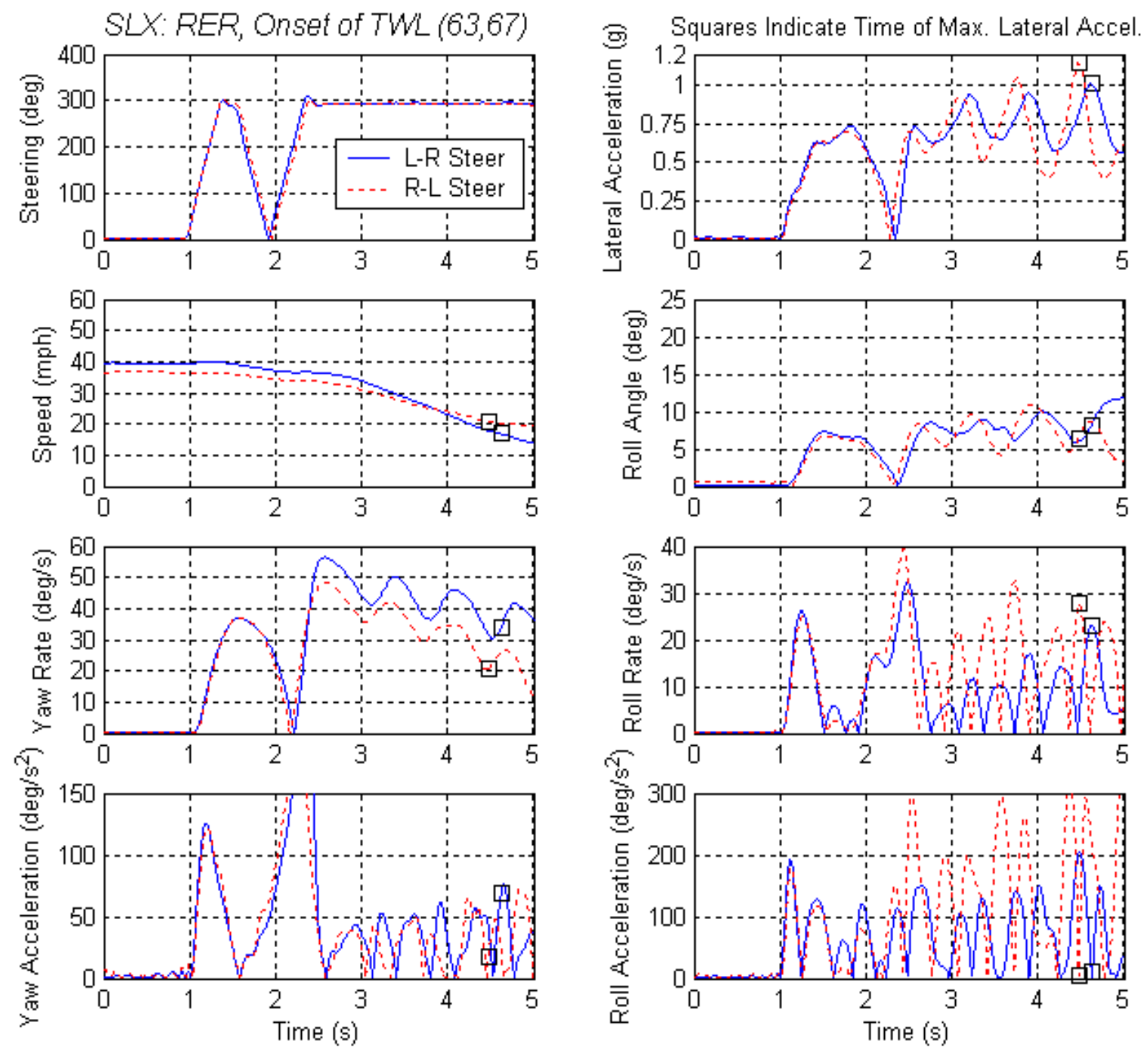

Figure 4.11: SLX, Nominal Load, Road Edge Recovery at Onset of TWL Left-Right Steer at $39.5 \mathrm{mph}$, Right-Left Steer at $36.4 \mathrm{mph}$ 

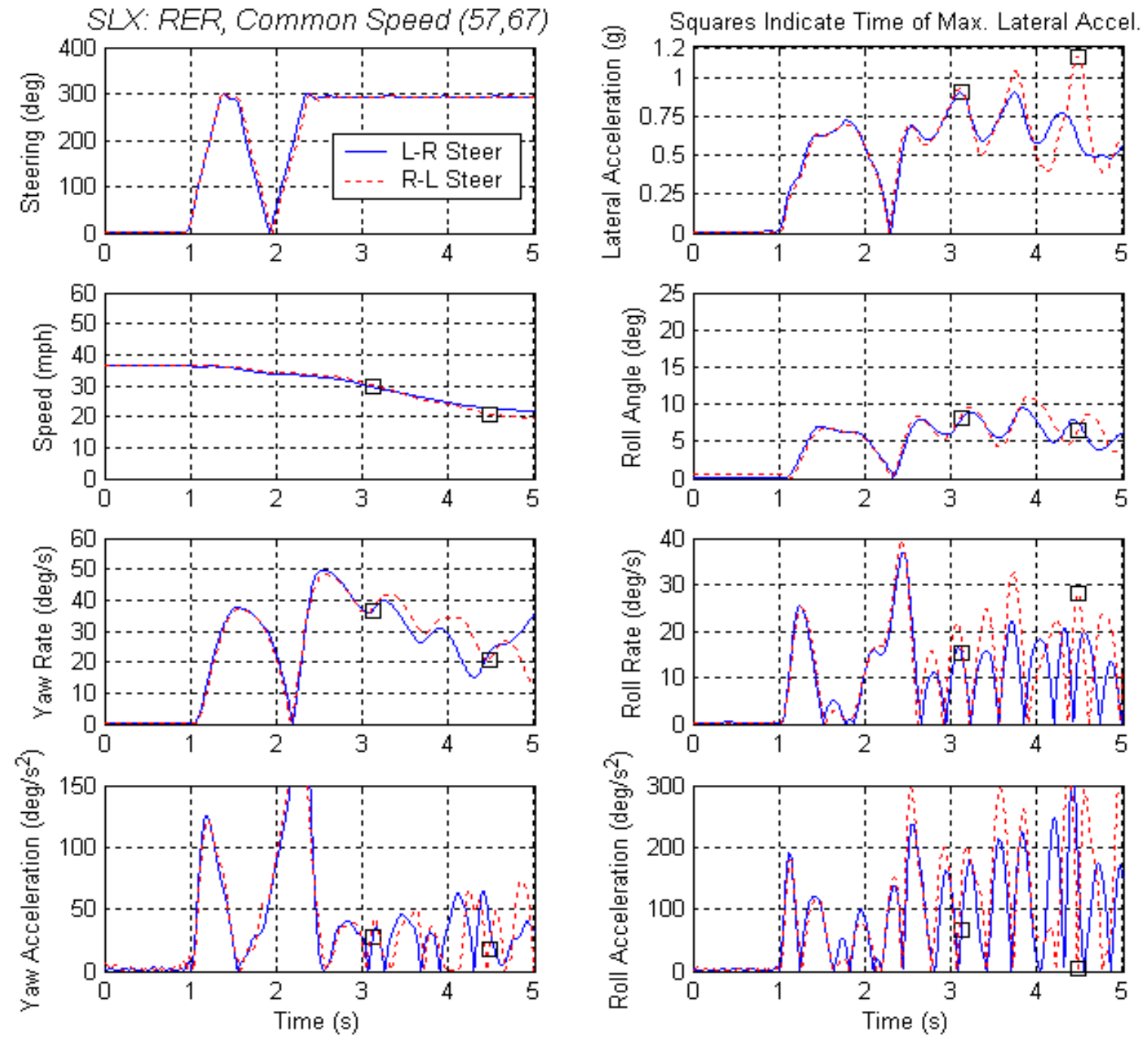

Figure 4.12: SLX, Nominal Load, Road Edge Recovery at Common Entrance Speed Left-Right Steer at $36.4 \mathrm{mph}$, Right-Left Steer at $36.4 \mathrm{mph}$ 

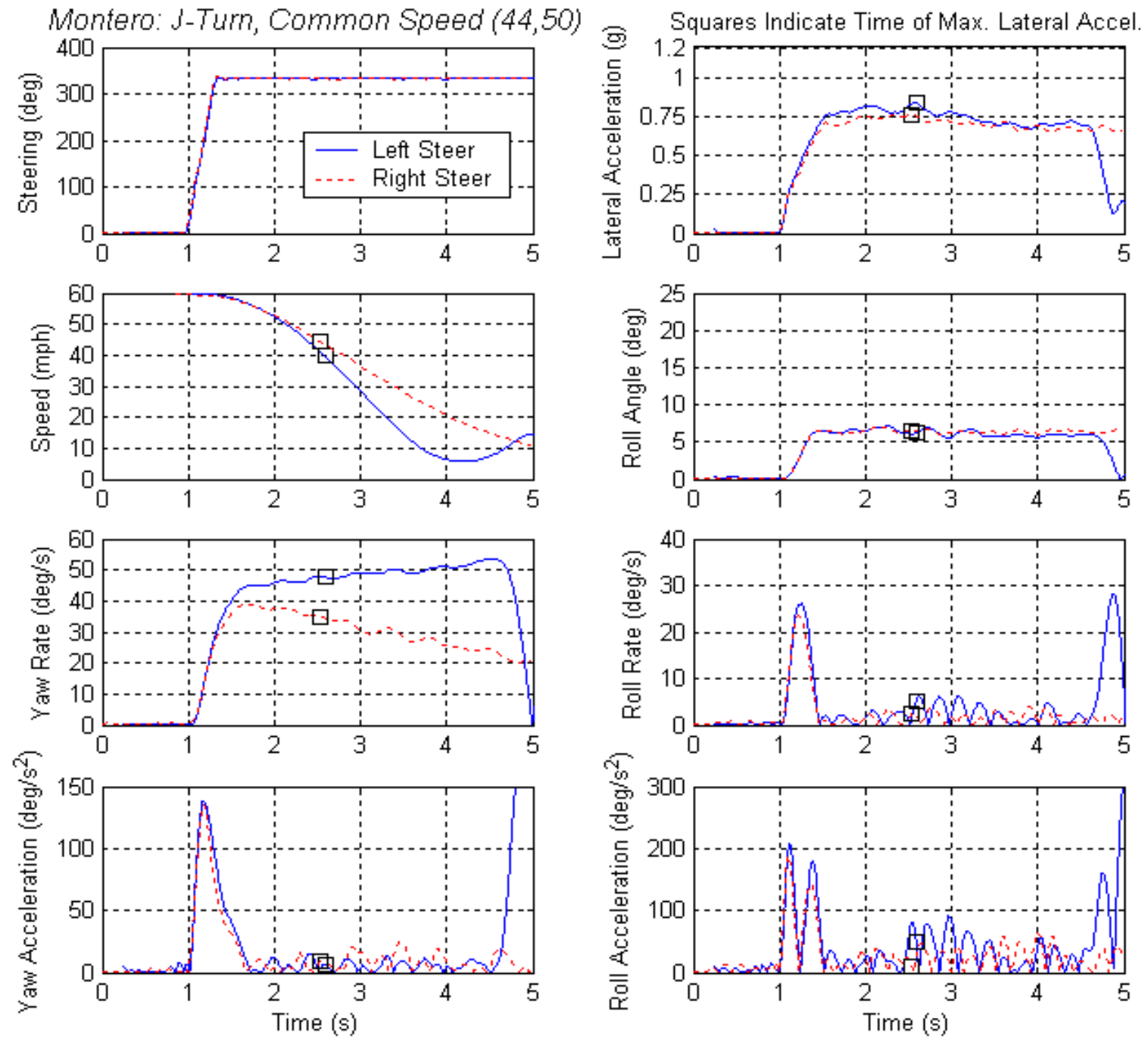

Figure 4.13: Montero, Nominal Load, J-turn at Common Entrance Speed* Left Steer at $60.3 \mathrm{mph}$, Right Steer at $59.9 \mathrm{mph}$

*Note TWL did not occur, thus the maximum speed was used. 

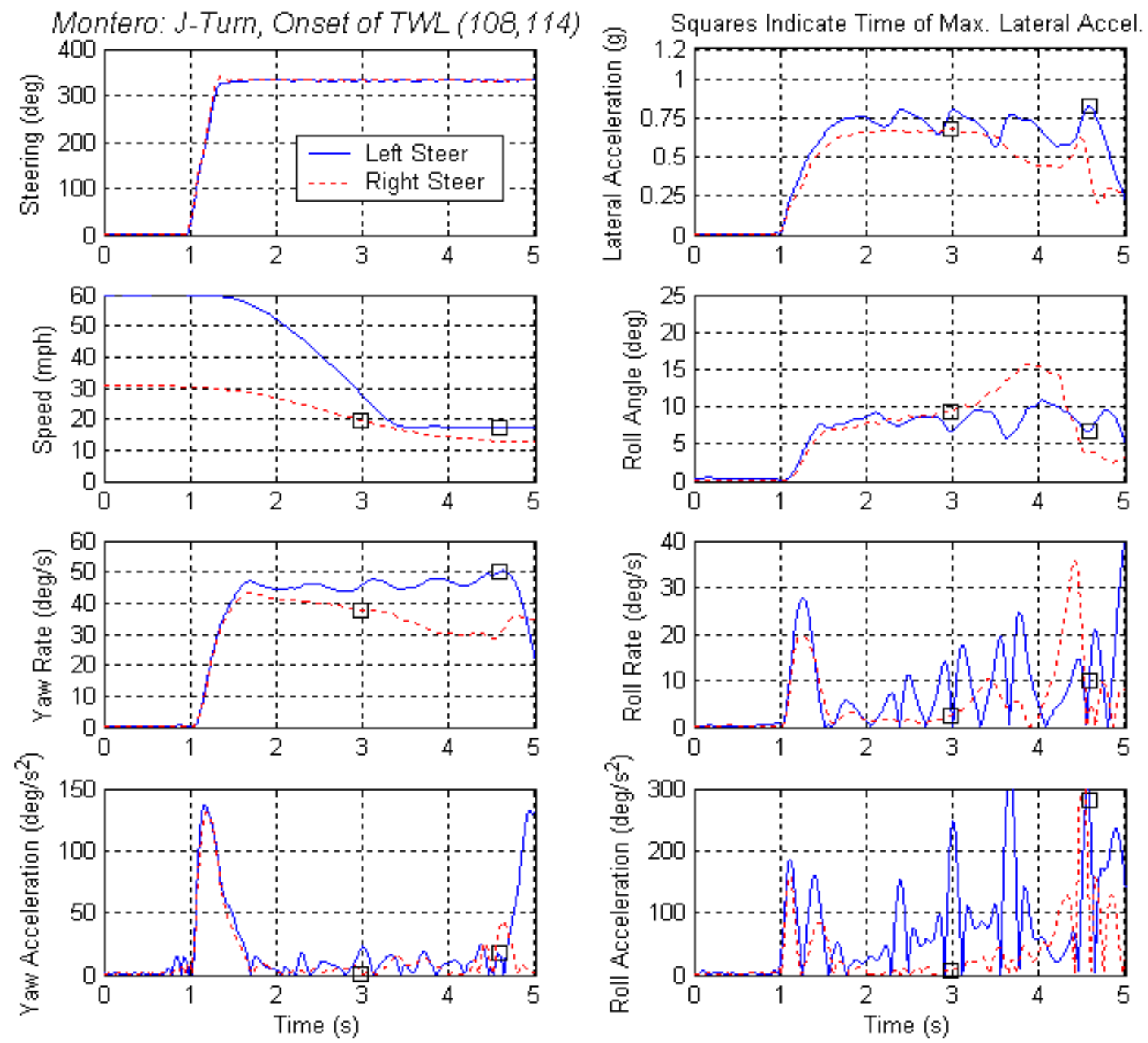

Figure 4.14: Montero, Maximum Occupancy, J-turn at Onset of TWL Left Steer at $60.1 \mathrm{mph}^{*}$, Right Steer at $30.6 \mathrm{mph}$

*Note TWL did not occur, thus the maximum speed was used. 

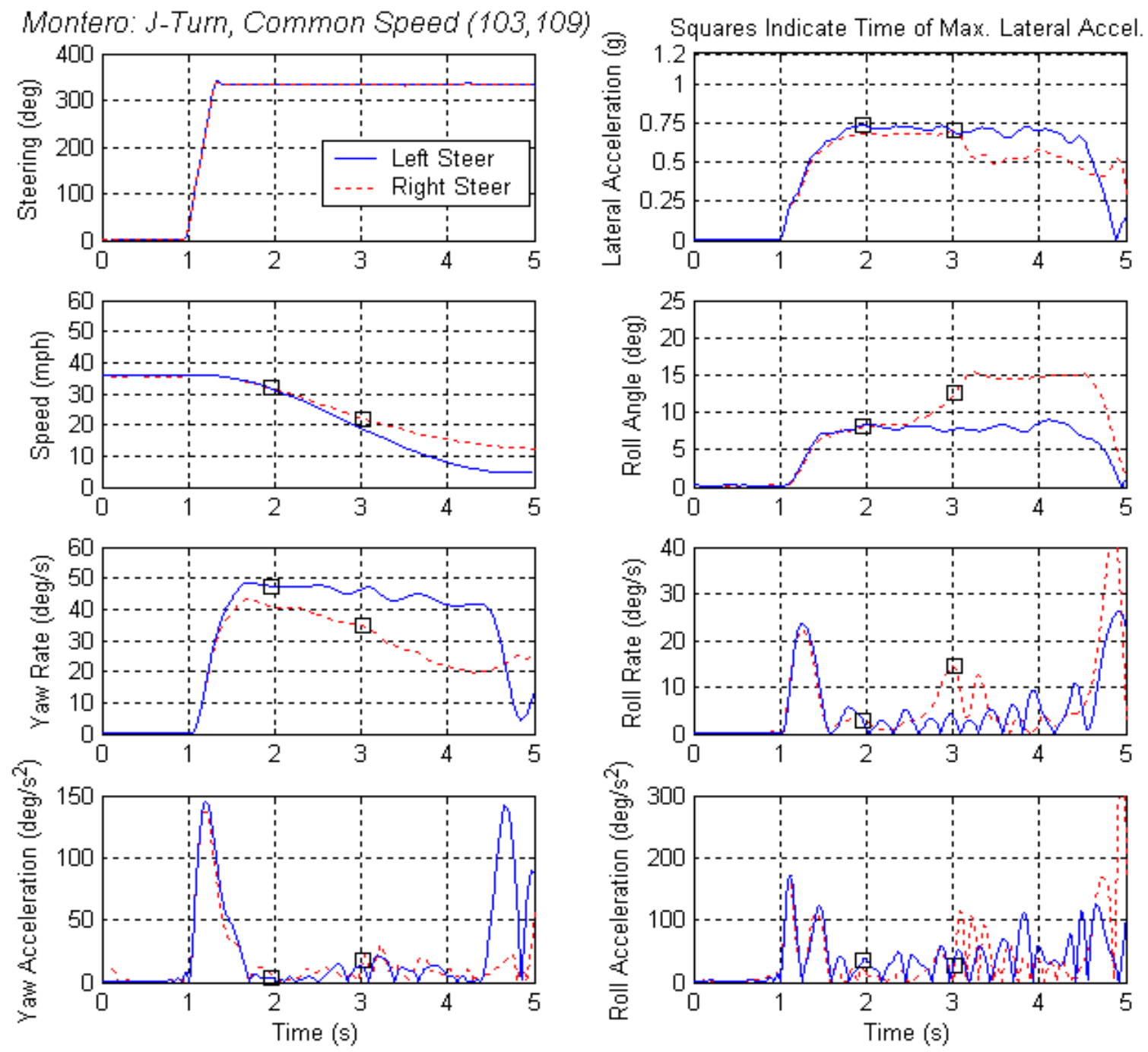

Figure 4.15: Montero, Maximum Occupancy, J-turn at Common Entrance Speed Left Steer at $36.1 \mathrm{mph}$, Right Steer at $35.6 \mathrm{mph}$ 

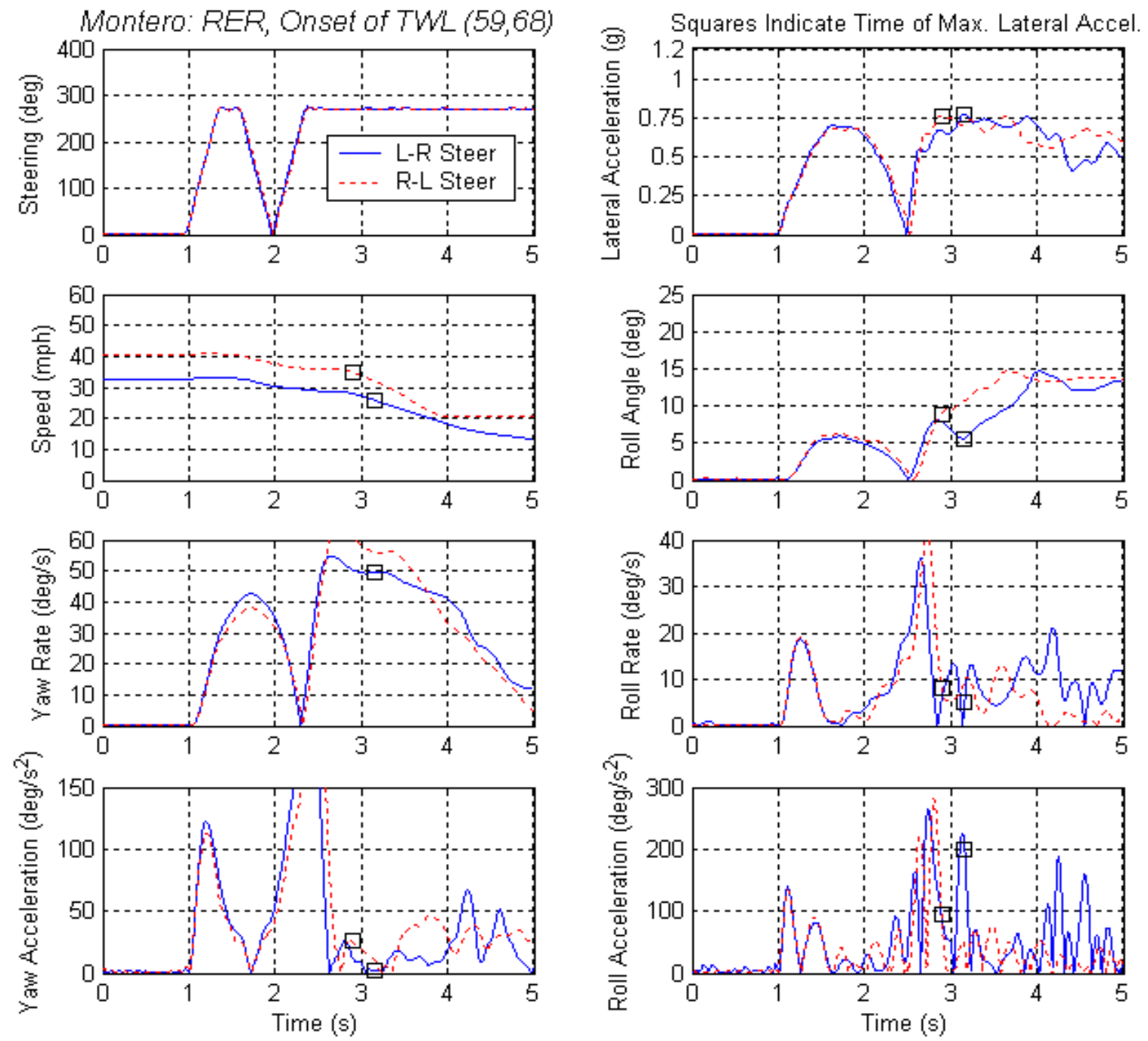

Figure 4.16: Montero, Nominal Load, Road Edge Recovery at Onset of TWL Left-Right Steer at $32.7 \mathrm{mph}$, Right-Left Steer at $40.6 \mathrm{mph}$ 

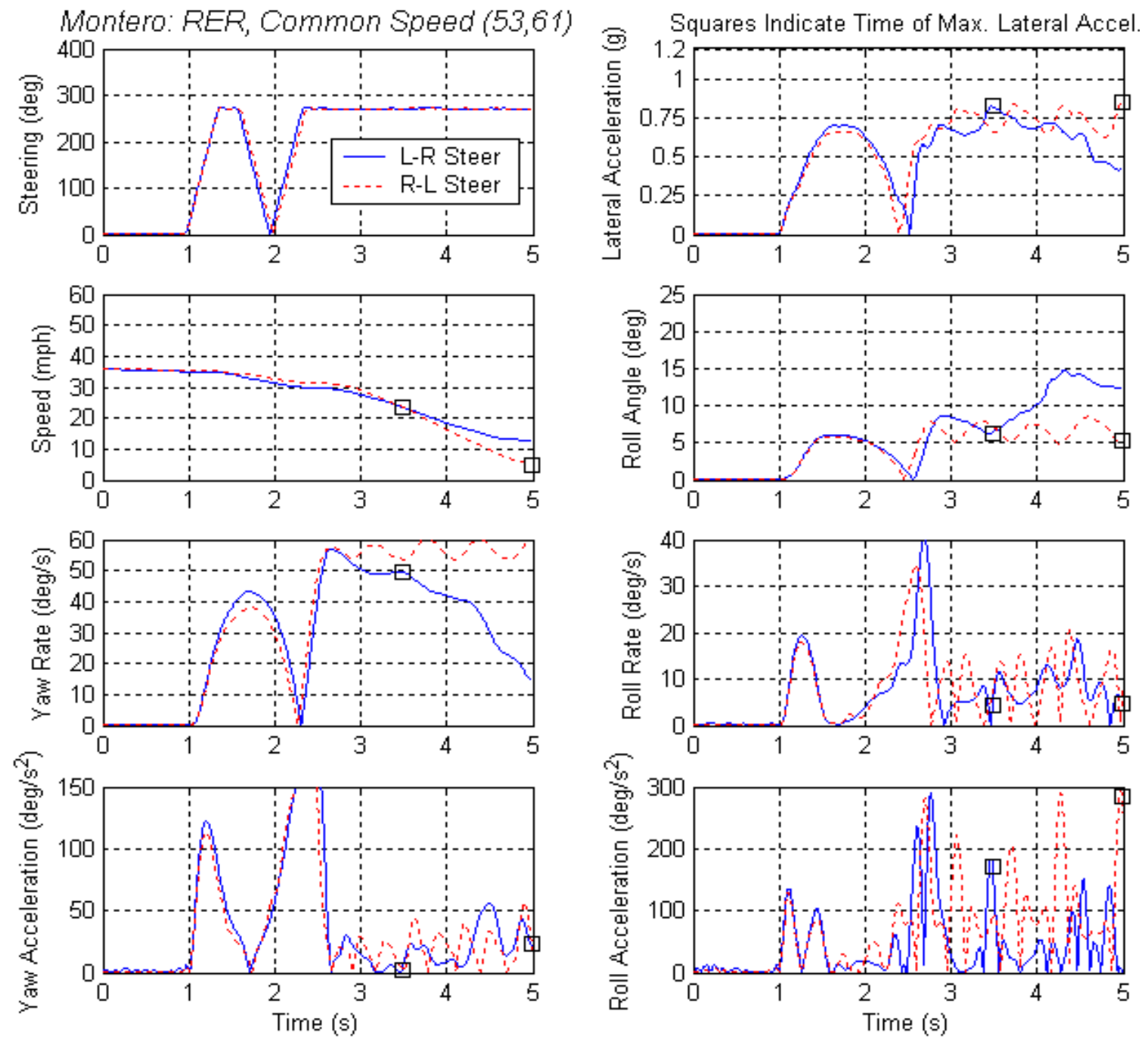

Figure 4.17: Montero, Nominal Load, Road Edge Recovery at Common Entrance Speed Left-Right Steer at $35.1 \mathrm{mph}$, Right-Left Steer at $35.6 \mathrm{mph}$ 
Aerostar: J-Turn, Common Speed (244,252)
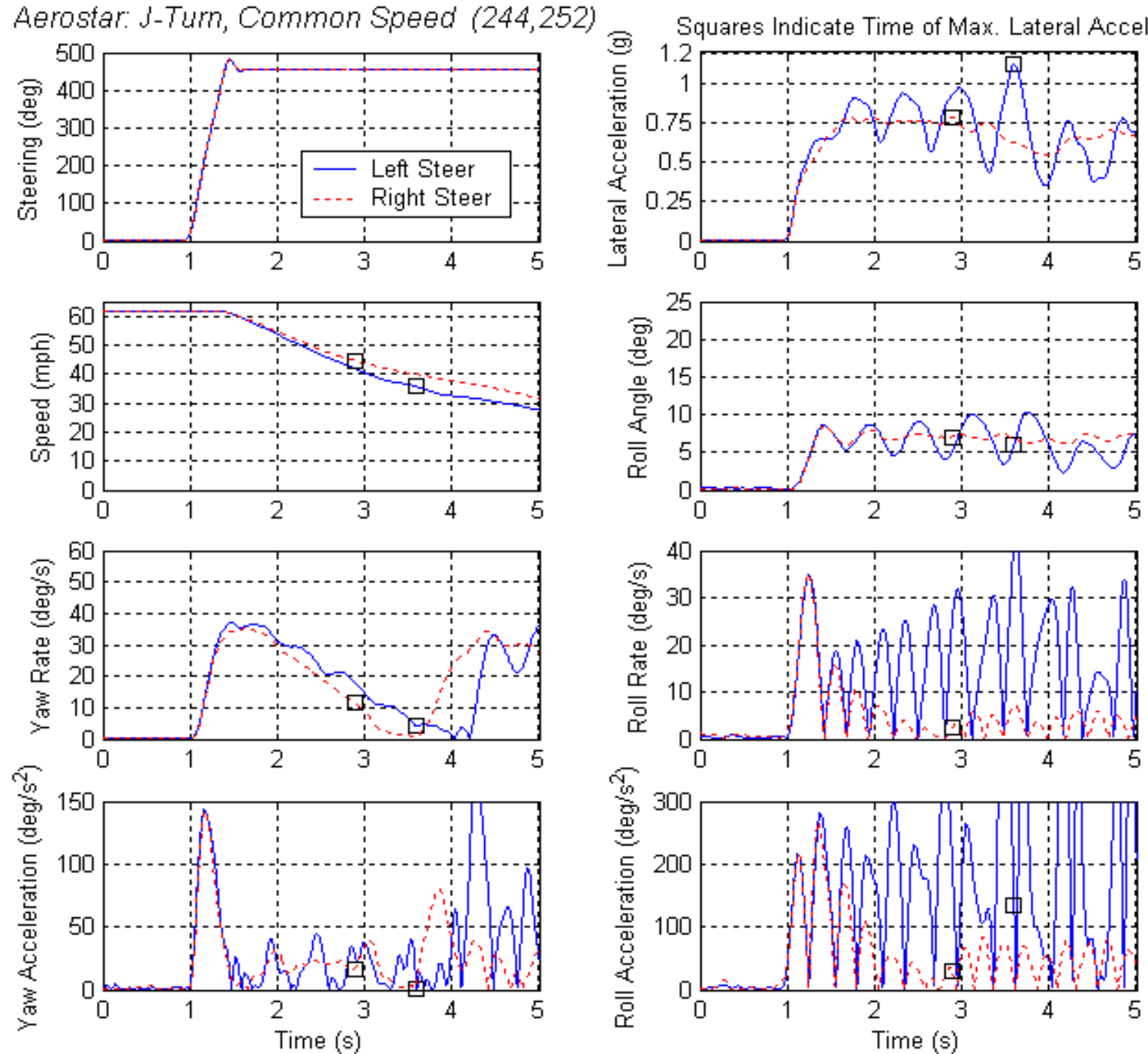

Figure 4.18: Aerostar, Nominal Load, J-turn at Common Entrance Speed* Left Steer at $61.6 \mathrm{mph}$, Right Steer at $61.6 \mathrm{mph}$

*Note TWL did not occur, thus the maximum speed was used. 

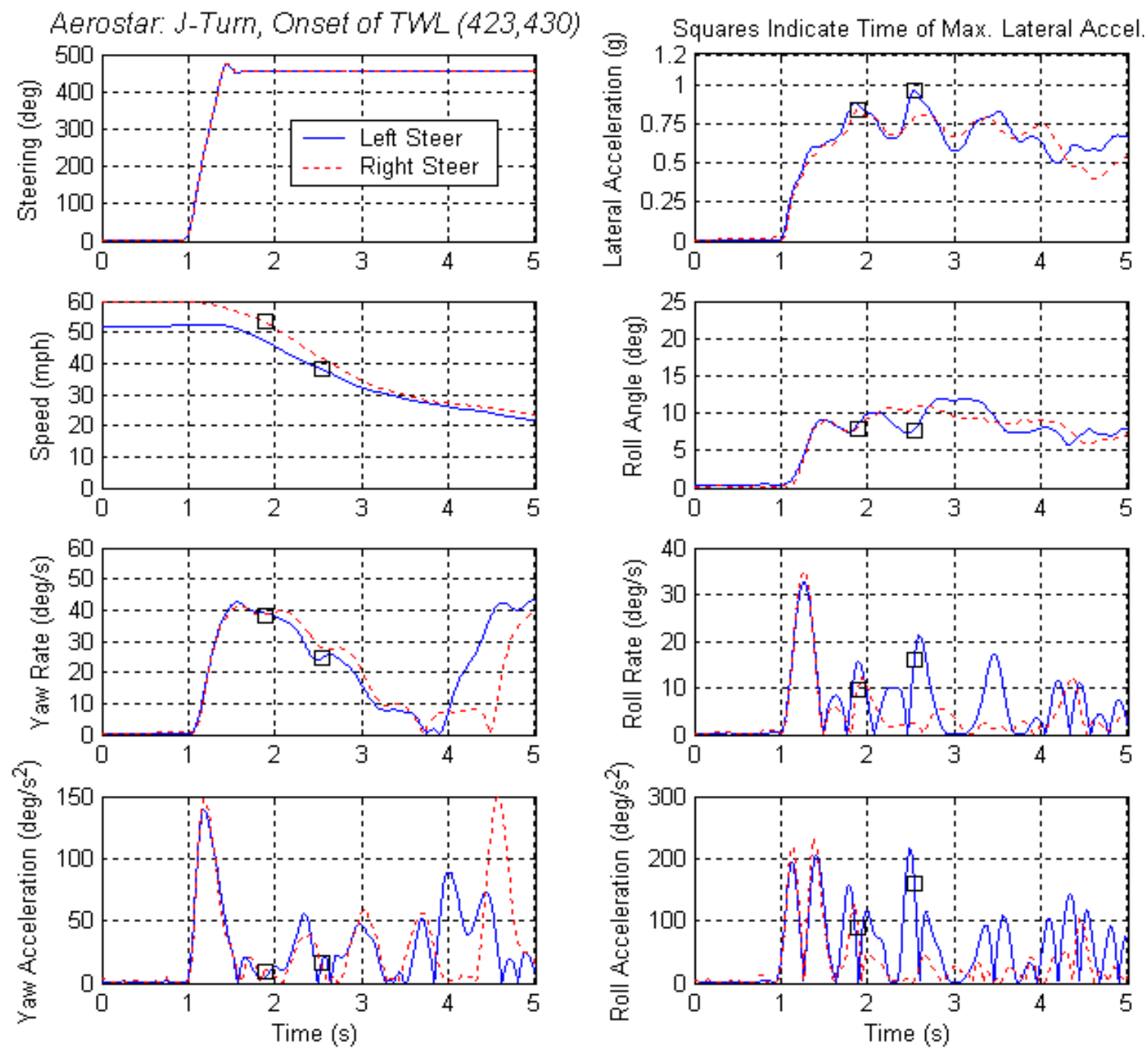

Figure 4.19: Aerostar, Maximum Occupancy, J-turn at Onset of TWL Left Steer at $52.1 \mathrm{mph}$, Right Steer at $59.7 \mathrm{mph}$ *

*Note TWL did not occur, thus the maximum speed was used. 

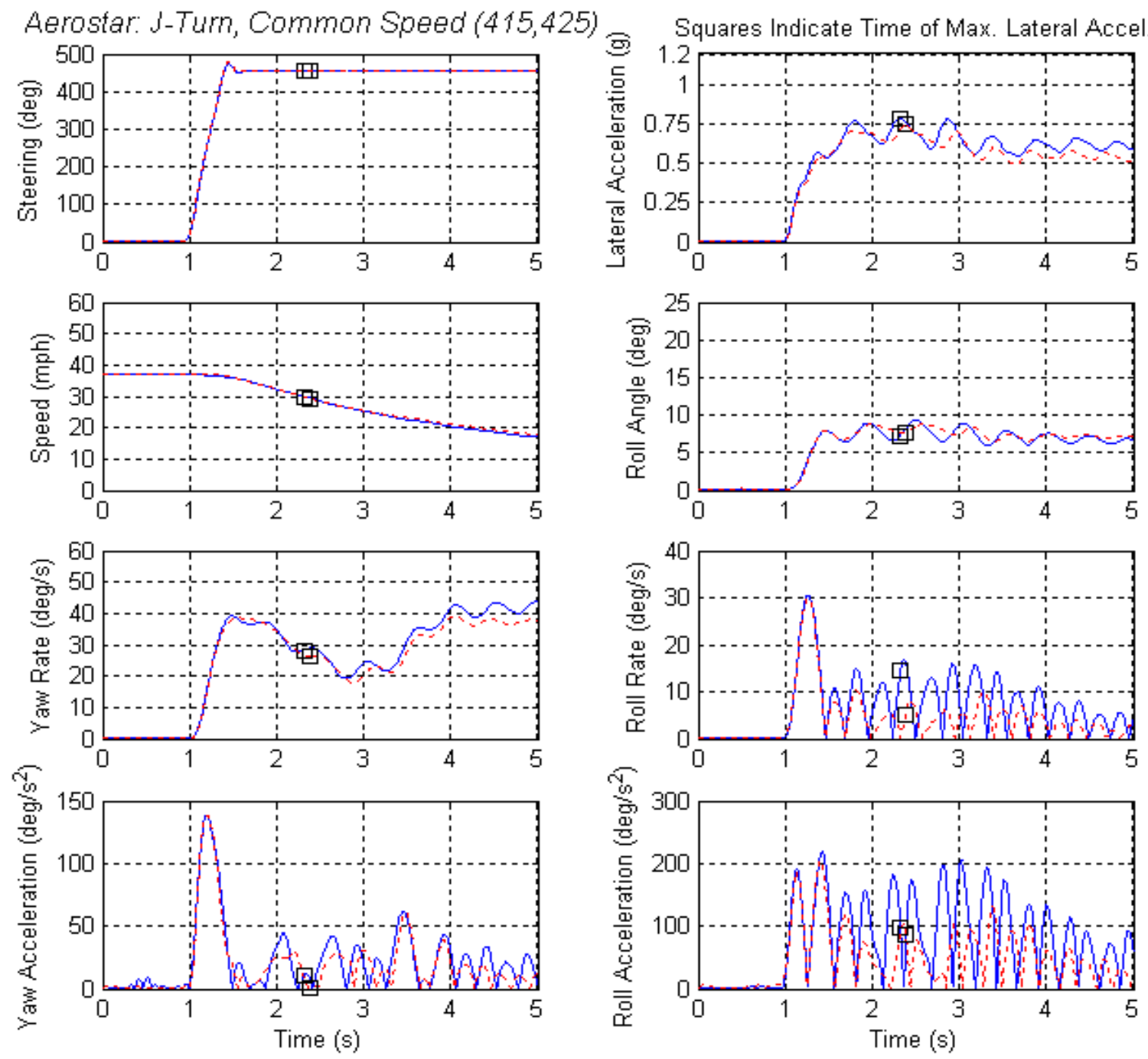

Figure 4.20: Aerostar, Maximum Occupancy, J-turn at Common Entrance Speed Left Steer at $37.1 \mathrm{mph}$, Right Steer at $37.0 \mathrm{mph}$ 

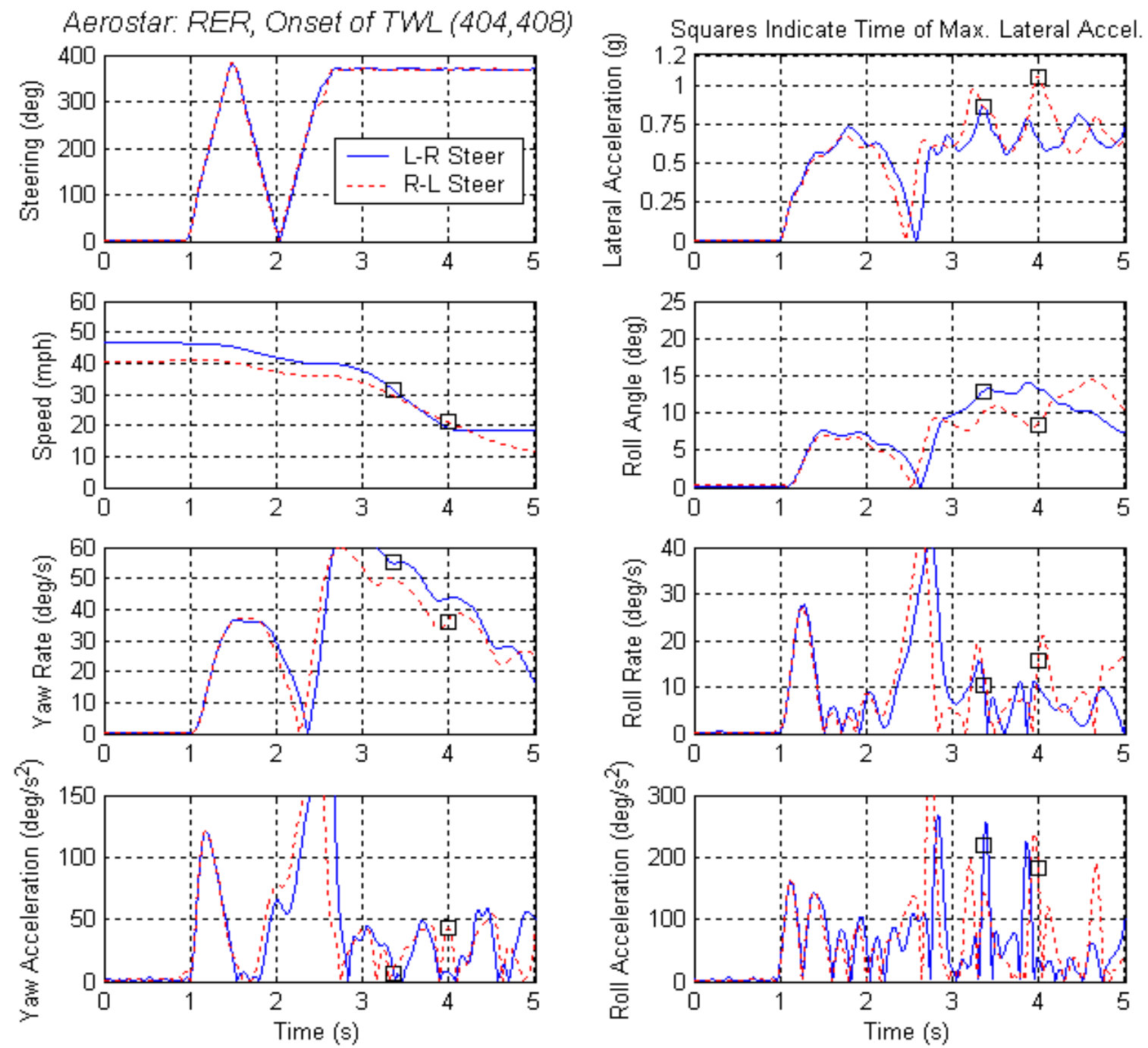

Figure 4.21: Aerostar, Maximum Occupancy, Road Edge Recovery at Onset of TWL Left-Right Steer at $46.5 \mathrm{mph}$, Right-Left Steer at $40.8 \mathrm{mph}$ 

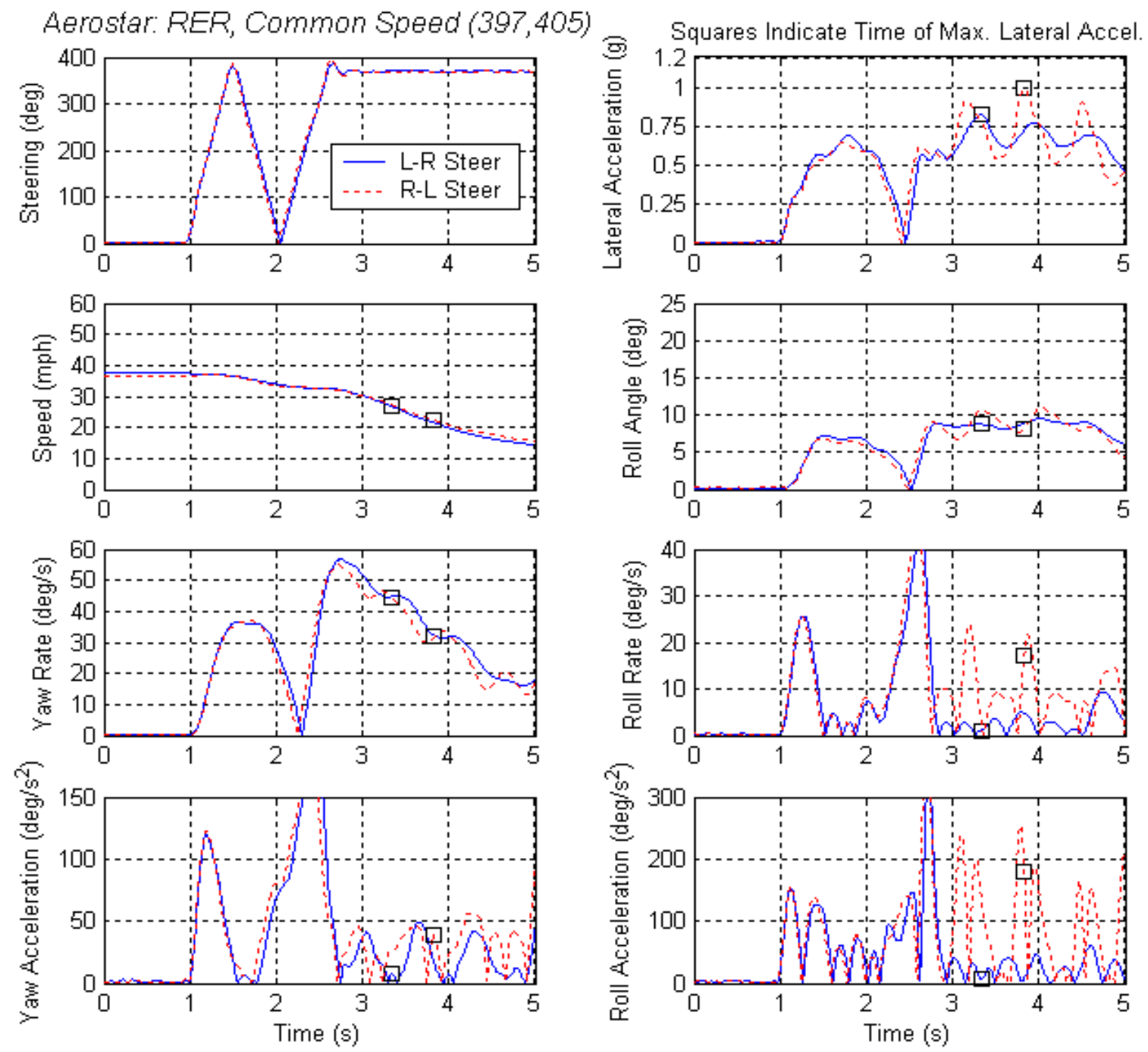

Figure 4.22: Aerostar, Maximum Occupancy,

Road Edge Recovery at Common Entrance Speed Left-Right Steer at $37.5 \mathrm{mph}$, Right-Left Steer at $36.6 \mathrm{mph}$ 
Section 4.2 presented many possible unintended differences in the vehicle inputs that may cause vehicle response asymmetries. The first input mentioned was the commanded steering inputs (handwheel position and rate). As shown in Table 4.1, the limits of the range of the handwheel rate achieved in the J-turn for the Montero and in the road edge recovery for the Aerostar had differences of $8.4 \%$ and $20.1 \%$, respectively. In Figure 4.14, the Montero's handwheel rate steering left in the J-turn was $1020.6 \mathrm{deg} / \mathrm{s}$ and steering right was $947.3 \mathrm{deg} / \mathrm{s}$ (7.4\% difference). In Figure 4.21, the Aerostar achieved a handwheel rate in the reversal steer of the road edge recovery of $720.2 \mathrm{deg} / \mathrm{s}$ for left-right maneuver and $680.8 \mathrm{deg} / \mathrm{s}$ for the right-left maneuver (5.6\% difference). These different handwheel rates (for all of the tests, not just those tests plotted in Figures 4.14 and 4.21) did not occur at the same maneuver entrance speed, therefore the effect cannot be singled out. However, all of the other tests shown in Figures $4.7-4.22$ had negligible differences in the handwheel angle and rates achieved with respect to the comparison being made in each individual figure.

The second input considered was the initial handwheel angle prior to the maneuver and any instrumentation offsets. The average for each data set shown in Figures $4.7-4.22$ for the second prior to the initial handwheel steer was used to determine if any initial offsets existed. For example, the average values for the first second shown in Figure 4.18 for the left steer J-turn were: $0.02^{\circ}$ handwheel angle, $0.02 \mathrm{~g}$ of lateral acceleration, $0.43^{\circ} / \mathrm{sec}$ of yaw, $0.32^{\circ}$ of body roll, etc. These values are comparable to the other tests, and therefore the effect of the initial handwheel angle prior to the maneuver and any instrumentation offsets is negligible. 
The next two inputs mentioned, the environmental conditions and the test surface, cannot be determined from the available data. However, as stated earlier, the effect on vehicle asymmetric response, if any, is negligible.

The fifth and sixth unintended inputs discussed were the effect of tire wear and worn/damaged suspension components. Given the severity of the tests, it is likely that significant tire and suspension wear occurred. Unfortunately, no measurements of tire wear (tread depth, tread shoulder wear and temperature, etc.) or suspension forces/deflections were made. The magnitude of these effects is less for the simpler, single steer, J-turn maneuver. For example, a left steer J-turn will scrub off some of the right shoulder of the tires. The next set of tests, right steer J-turn, will be on those same used tires, but the effective part of the tire for these tests, the left shoulder, will be relatively unworn compared to the right shoulder. This idea of minimized effect of the tire wear also applies to some extent to the suspension components, e.g., bump stops. Therefore, the J-turn maneuver is considered to be more reliable in indicating vehicle asymmetric response.

The last item presented in Section 4.2, test-to-test variability, is an unfortunate occurrence in real world testing. Although its effect cannot be determined, Phase VI tests were repeated to verify the minimum maneuver entrance speed for which TWL was produced. As such, those speeds listed in Table 2.2 are a clear indication of vehicle asymmetric response. 


\section{CHAPTER 5}

\section{SUSPENSION AND STEERING TESTS}

\subsection{Introduction}

The fifth chapter accomplishes the fourth objective of this research, which is to identify any vehicle components/characteristics, such as steering system or suspension asymmetries that may cause vehicle asymmetric response. Suspension and steering tests were performed at S.E.A., Inc. in Columbus, Ohio. As discussed in Chapter 4, the J-turn maneuver is considered to be more reliable in indicating vehicle asymmetric response. In addition, the J-turn is a simpler maneuver relative to the road edge recovery; therefore, modeling the dynamics of the J-turn and determining the effect of suspension and/or steering asymmetries is more intuitive for the J-turn. Since the SLX and the Montero exhibited the most differences in left versus right steer J-turn maneuvers, they were chosen for the suspension and steering tests. A description of these tests along with the results is given in this chapter.

\subsection{Description of Suspension and Steering Tests}

The following types of tests were performed on the SLX and the Montero: bounce, roll, lateral and longitudinal force compliance, steering compliance, and steering ratio. Each type of test was performed in the nominal load configuration, and some 
of the tests were repeated for the maximum occupancy load configuration. Typically the compliance tests are performed for one side of the vehicle (e.g. left side longitudinal force compliance), because the other (right) side is assumed to respond identically. However, the SLX and Montero were not assumed to respond symmetrically, so both left and right side tests were performed when applicable. The actual tests performed with respect to the type of test, side of vehicle, and load configuration will be presented in Section 5.3.1 for the SLX and 5.3.2 for the Montero.

During the quasi-static bounce and roll tests, steel cross members were rigidly attached to the chassis of the vehicle. The ends of the cross members (at the sides of the vehicle) were moved up and down in phase to drive the vehicle chassis through its range of bounce motion and out of phase to drive the chassis through its range of roll motion. During the tests, the tires were positioned on free-floating wheel-pad units (commonly referred to as "Hunter pads") so that no lateral or longitudinal forces, or aligning moments would exist at the tires. For all but the steering ratio test, the steering wheel was locked, and the service and park brakes were applied. During the tests, eleven string potentiometers, six linear displacement transducers, four vertical load scales, an inclinometer, and a load cell were used to measure the quantities necessary to describe the suspension or steering behavior. The transducer signals were appropriately processed to provide the desired engineering quantities.

A pneumatic cylinder was used to generate lateral and longitudinal forces for the translational compliance tests. The lateral compliance tests involved pulling either the right or left wheel pad toward the opposite side of the vehicle using the pneumatic cylinder and a custom attachment to the chassis. The out-of-phase longitudinal 
compliance test involved pulling the same side front and rear wheel pads together using the pneumatic cylinder. The steering compliance test involved applying a pure aligning moment to the wheel under consideration using a custom apparatus mounted to the wheel. For all the compliance tests, the forces were measured using a load cell, and the necessary string potentiometers, displacement transducers, and load scales were used to measure the quantities necessary to describe the suspension characteristics under study. The steering ratio test was performed with the engine running, so that the effect of power steering would be included.

All wheel position and orientation measurements were made on both the right and left sides of the vehicle. The standard SAE vehicle dynamics coordinate system (longitudinal X-axis positive forward, lateral Y-axis positive rightward, and vertical Zaxis positive downward) was used to define the directions of the measured quantities. All quantities are measurements made relative to the 'normal' vehicle state prior to testing. For example, the vertical force shown on the graphs is the change in vertical force, not the absolute value of the vertical force. The measured translational quantities are positive directed along the positive $\mathrm{X}, \mathrm{Y}$, and $\mathrm{Z}$ axes. For example, a positive lateral deflection or force indicates a deflection or force directed to the right. Similarly for rotational quantities, positive rotations and applied torques are defined positive about an axis defined using the right-hand screw rule. Vertical suspension deflection (chassis deflection less tire deflection) is defined as positive for suspension compression and negative for suspension rebound. 


\subsection{Results of the Suspension and Steering Tests}

The Suspension Test Summary tables (5.2 for the SLX and 5.4 for the Montero) contain selected suspension and steering system parameter results. These results are from linear curve fits to the listed suspension characteristic curves. The ranges of the characteristic curves over which the linear curve fits were made are shown in the corresponding graphs following the summary table. For all the graphs, the circles indicate the measured data points, the dashed lines connect the circles to represent the respective characteristic behavior throughout the full test range, and solid lines represent curve fits. For graphs with both left and right side suspension plots (e.g. front suspension spring rate and roll steer), circles are used for the right side suspension characteristics and triangles for the left side.

For brevity, only those graphs that indicate left versus right side suspension asymmetric response are shown, but all graphs corresponding to the steering system (front steering compliance and steering ratio) are shown. Note that unless stated otherwise, plots are based on the nominal load configuration. 


\subsubsection{SLX tests}

Table 5.1 lists the tests performed on the SLX (DNP denotes “did not perform” test). As shown in Table 5.1, "Side” refers to the left (L) or right (R) side of the vehicle tested. These tests only measure one side of the vehicle at a time, unlike the roll, bounce, and steering ratio tests.

\begin{tabular}{|c|c|c|c|}
\hline \multirow{2}{*}{ Test } & Side & \multicolumn{2}{|c|}{ Load Configuration } \\
\cline { 2 - 4 } & $\mathrm{L} / \mathrm{R}$ & Nominal & Max Occ \\
\hline Front Bounce & & $\mathrm{x}$ & $\mathrm{DNP}$ \\
\hline Rear Bounce & & $\mathrm{x}$ & $\mathrm{DNP}$ \\
\hline Front Roll & & $\mathrm{x}$ & $\mathrm{x}$ \\
\hline Rear Roll & & $\mathrm{x}$ & $\mathrm{x}$ \\
\hline Front Lateral Compliance & $\mathrm{L}$ & $\mathrm{x}$ & $\mathrm{x}$ \\
\hline Front Lateral Compliance & $\mathrm{R}$ & $\mathrm{x}$ & $\mathrm{DNP}$ \\
\hline Rear Lateral Compliance & $\mathrm{L}$ & $\mathrm{DNP}$ & $\mathrm{x}$ \\
\hline Rear Lateral Compliance & $\mathrm{R}$ & $\mathrm{x}$ & $\mathrm{x}$ \\
\hline Out-of-Phase Longitudinal Compliance & $\mathrm{L}$ & $\mathrm{x}$ & $\mathrm{DNP}$ \\
\hline Out-of-Phase Longitudinal Compliance & $\mathrm{R}$ & $\mathrm{x}$ & $\mathrm{DNP}$ \\
\hline Front Steering Compliance & $\mathrm{L}$ & $\mathrm{x}$ & $\mathrm{x}$ \\
\hline Front Steering Compliance & $\mathrm{R}$ & $\mathrm{x}$ & $\mathrm{DNP}$ \\
\hline Rear Steering Compliance & $\mathrm{L}$ & $\mathrm{x}$ & $\mathrm{x}$ \\
\hline Rear Steering Compliance & $\mathrm{R}$ & $\mathrm{x}$ & $\mathrm{DNP}$ \\
\hline Steering Ratio Test & & $\mathrm{x}$ & $\mathrm{DNP}$ \\
\hline
\end{tabular}

Table 5.1: SLX Suspension and Steering Tests 


\begin{tabular}{|c|c|c|}
\hline \multicolumn{3}{|c|}{ SLX Front Bounce Test Results } \\
\hline & Front Right & Front Left \\
\hline $\begin{array}{l}\text { Front Ride Rate } \\
\text { Vertical Force vs. Suspension Deflection }\end{array}$ & $160.2 \mathrm{lb} / \mathrm{in}$ & $159.0 \mathrm{lb} / \mathrm{in}$ \\
\hline $\begin{array}{l}\text { Front Bump Stop Stiffness* } \\
\text { Vertical Force vs. Suspension Deflection }\end{array}$ & $\begin{array}{l}260 \mathrm{lb} / \mathrm{in} \\
1172 \mathrm{lb} / \mathrm{in}\end{array}$ & $\begin{array}{l}365 \mathrm{lb} / \mathrm{in} \\
1447 \mathrm{lb} / \mathrm{in}\end{array}$ \\
\hline $\begin{array}{l}\text { Front Tire Static Stiffness (30 psi) } \\
\text { Vertical Force vs. Tire Deflection }\end{array}$ & 1352 lb/in & $1291 \mathrm{lb} / \mathrm{in}$ \\
\hline \multicolumn{3}{|c|}{ *Nonlinear, broken up and linearized for 1-2" and 2-3" suspension compression } \\
\hline \multicolumn{3}{|c|}{ SLX Rear Bounce Test Results } \\
\hline & Rear Right & Rear Left \\
\hline $\begin{array}{l}\text { Rear Ride Rate } \\
\text { Vertical Force vs. Suspension Deflection }\end{array}$ & $237.9 \mathrm{lb} / \mathrm{in}$ & $246.9 \mathrm{lb} / \mathrm{in}$ \\
\hline $\begin{array}{l}\text { Rear Bump Stop Stiffness* } \\
\text { Vertical Force vs. Suspension Deflection }\end{array}$ & $\begin{array}{l}395 \mathrm{lb} / \mathrm{in} \\
1517 \mathrm{lb} / \mathrm{in}\end{array}$ & $\begin{array}{l}423 \mathrm{lb} / \mathrm{in} \\
1910 \mathrm{lb} / \mathrm{in}\end{array}$ \\
\hline $\begin{array}{l}\text { Rear Tire Static Stiffness (35 psi) } \\
\text { Vertical Force vs. Tire Deflection }\end{array}$ & 1412 lb/in & $1427 \mathrm{lb} / \mathrm{in}$ \\
\hline
\end{tabular}

*Nonlinear, broken up and linearized for 0.5-1.6” and 1.6-2.2” suspension compression (continued)

Table 5.2: SLX Suspension Test Summary 
Table 5.2, continued

\section{SLX Front Roll Test Results}

First value given is Nominal Load Case and the other value is Maximum Occupancy Case

\begin{tabular}{|l|c|c|}
\hline & Front Right & Front Left \\
\hline Front Roll Steer & 0.0269 & 0.0571 \\
Wheel Steer Angle vs. Roll Angle & 0.0085 & 0.0330 \\
\hline Front Roll Camber & 0.846 & 0.839 \\
Wheel Camber Angle vs. Roll Angle & 0.826 & 0.832 \\
\hline Overall Front Roll Rate & \multicolumn{2}{|c|}{7703 in-lb/deg } \\
Roll Moment vs. Roll Angle & \multicolumn{2}{|c|}{8218 in-lb/deg } \\
\hline
\end{tabular}

\section{SLX Rear Roll Test Results}

First value given is Nominal Load Case and the other value is Maximum Occupancy Case

\begin{tabular}{|l|c|c|}
\hline & Rear Right & Rear Left \\
\hline Rear Roll Steer & 0.0518 & 0.0516 \\
Wheel Steer Angle vs. Roll Angle & 0.0252 & 0.0292 \\
\hline Rear Roll Camber & 0.0865 & 0.0845 \\
Wheel Camber Angle vs. Roll Angle & 0.112 & 0.128 \\
\hline Overall Rear Roll Rate & \multicolumn{2}{|c|}{4754 in-lb/deg } \\
Roll Moment vs. Roll Angle & \multicolumn{2}{|c|}{5832 in-lb/deg } \\
\hline
\end{tabular}

(continued) 
Table 5.2, continued

\section{SLX Front Steering Compliance Test Results}

First value given is Nominal Load Case and the other value is Maximum Occupancy Case

\begin{tabular}{|l|c|c|}
\hline & Front Right & Front Left \\
\hline $\begin{array}{l}\text { Front Steering Compliance (deg/(ft-lb)) } \\
\text { Applied Steering Torque vs. Steer Angle }\end{array}$ & $\begin{array}{c}0.00501 \\
\text { DNP }\end{array}$ & $\begin{array}{c}0.00452 \\
0.00451\end{array}$ \\
\hline \multicolumn{3}{|c|}{ SLX Steering Ratio Test Results } \\
\hline \multicolumn{2}{|c|}{ Right } & Left \\
\hline $\begin{array}{l}\text { Steering Ratio (fit between } \pm 360^{\circ} \text { ) } \\
\text { Steering Wheel Angle vs. Front Wheel Steer Angle }\end{array}$ & 19.76 & 19.85 \\
\hline $\begin{array}{l}\text { Ave. Steering Ratio (fit between } \pm 360^{\circ} \text { ) } \\
\text { Average of Right and Left Measured Ratios }\end{array}$ & 19.80 \\
\hline
\end{tabular}


The results of the front bounce test are shown in Figure 5.1. The linear suspension spring rate was computed based on the suspension deflection from -1.0 to 1.0 inch during the front bounce test. All of the static tire stiffnesses (front and rear of both vehicles) were computed over the range of -0.5 to 0.5 inches of vertical tire deflection. The nonlinear response beyond 1.5 inches of suspension compression is an indication of front bump stop contact with the chassis. This nonlinear region is simply referred to as the bump stop stiffness. The front bump stops were short rubber squares, as shown in the Figure 5.2. The front suspension was a typical torsion bar suspension with a stabilizer bar. Refer to Figure 5.11 for a diagram of the front suspension.

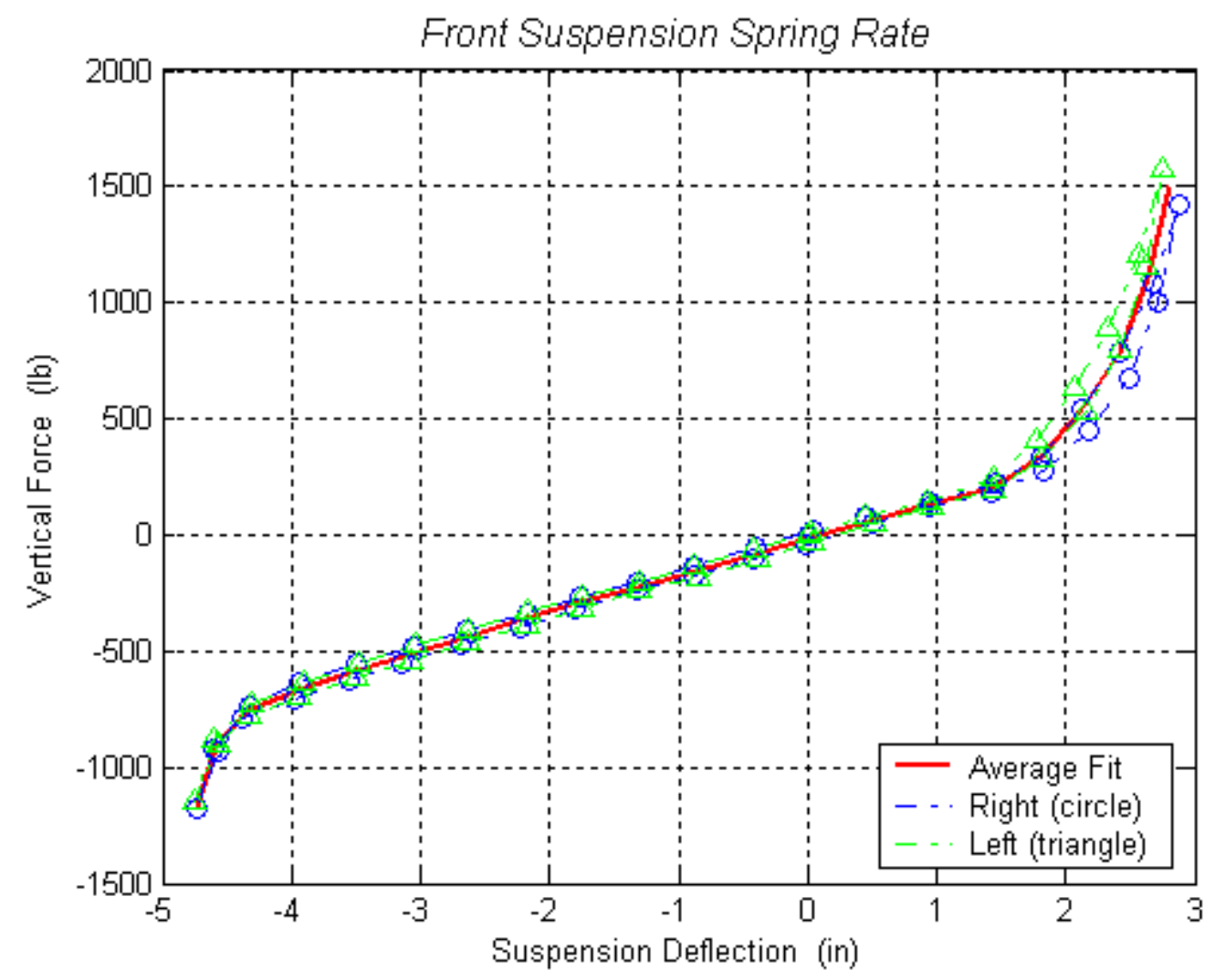

Figure 5.1: SLX Front Suspension Spring Rate 


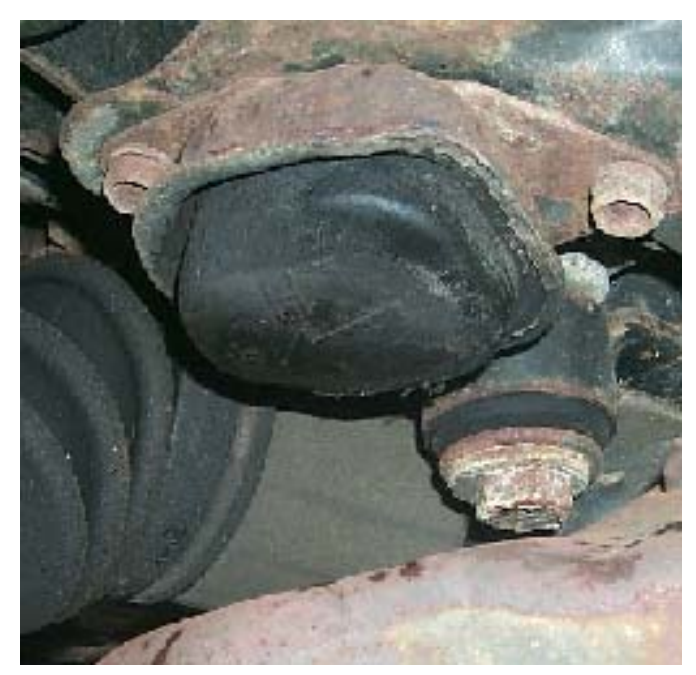

Figure 5.2: SLX Front Bump Stop

As stated in Table 5.2, the linearized front bump stop stiffness from 1 to 2 inches of suspension compression is $365 \mathrm{lb} / \mathrm{in}$ and $260 \mathrm{lb} / \mathrm{in}$ for the left and right side respectively, and from 2 inches to maximum compression measured, the stiffness is 1445 lb/in and $1172 \mathrm{lb} /$ in for the left and right side respectively.

The results of the rear bounce test are shown in Figure 5.3. The linear suspension spring rate was computed based on the suspension deflection from -1.0 to 1.0 inch. Note the nonlinear bump stop stiffness (about 0.5 to 2.1 inches of suspension deflection). During testing, the rear bump stops contacted the axle at 0.5 inches of suspension compression. A sketch of the rear bump stops is shown in Figure 5.4. The rear suspension consisted of a solid axle with coil springs, stabilizer bar, lateral rod, and trailing links. Refer to Figure 5.12 for a diagram of the rear suspension. 


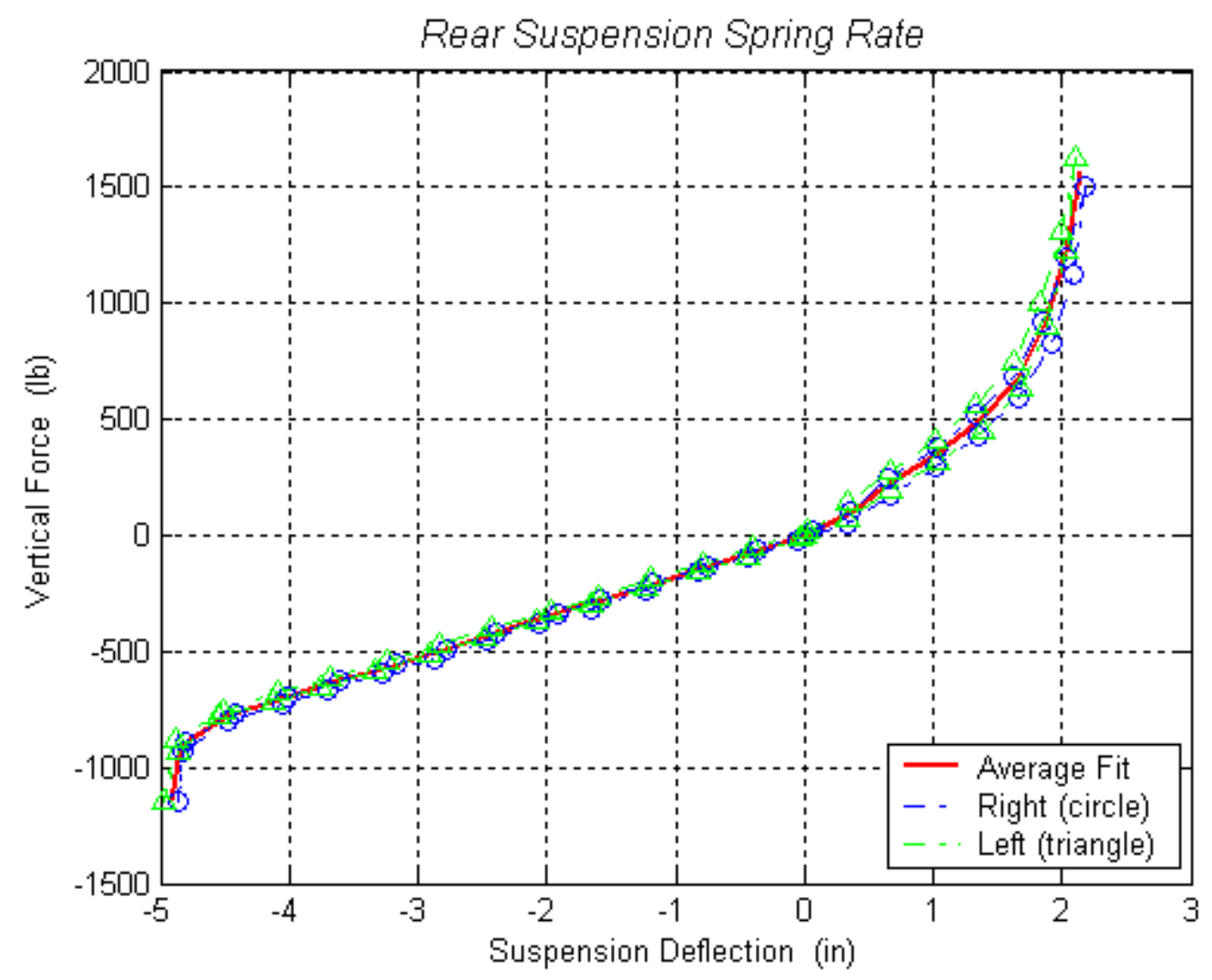

Figure 5.3: SLX Rear Suspension Spring Rate

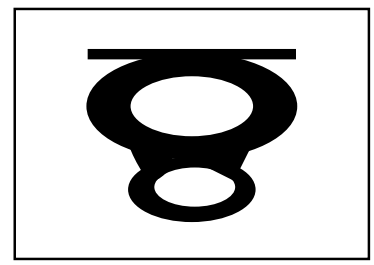

Figure 5.4: Sketch of SLX Rear Bump Stop (side view)

The linearized bump stop stiffness from 0.5 to 1.6 inches of suspension compression is $423 \mathrm{lb} / \mathrm{in}$ and $395 \mathrm{lb} / \mathrm{in}$ for the left and right side respectively, and from 1.6 inches to the maximum compression measured, the stiffness is $1910 \mathrm{lb} / \mathrm{in}$ and 1517 $\mathrm{lb} /$ in for the left and right side respectively. 
Although the springs, shock absorbers, and anti-roll bars are always active regardless of the direction of steer or roll angle, the bump stops only have an effect upon contact (i.e., in a severe maneuver), and therefore the bump stops on only one side of the vehicle can be active at a time (unless the vehicle is loaded beyond GVWR). Given that, and the differences in the right versus left bump stop stiffnesses (both front and rear), severe body roll (where the bump stops are compressed) will be greater clockwise (CW), i.e., in a left turn. The differences in the bump stop stiffnesses are likely one of the reasons the SLX experiences TWL at a lower maneuver entrance speed in a left steer versus a right steer J-turn maneuver. The stiffer bump stops absorb more of the energy as the vehicle rolls. During a severe maneuver, the bump stops may be completely compressed, at which point they act as a rigid link connecting the body (sprung mass) to the suspension (unsprung mass). The stiffer side of the suspension will suppress the roll acceleration such that less of an impact will occur when the bump stop is completely compressed. The softer side of the suspension will allow more roll acceleration and thus more of an impact will occur from the momentum of the sprung mass. Upon this faster, harder impact, the body may either rebound abruptly or continue to roll due to the sprung mass momentum.

As mentioned earlier, the nonlinear response region of suspension compression is simply referred to as the bump stop stiffness, and therefore, is not the actual stiffness of the bump stops. The suspension kinematics affect how the suspension deflects under lateral, longitudinal, and vertical load. For instance, the increased stiffness of the left side suspension (Figures 5.1 and 5.3) is likely due to earlier bump stop contact, and therefore, simply replacing or switching the bump stops will not have any effect on the vehicle 
response. In addition to the bounce tests, this phenomenon will also affect the roll tests, in particular the roll stiffness of the SLX in CW versus CCW (counter-clockwise) roll. Other vehicle bounce test data (2000 Kia Sportage and 1998 Chevrolet Malibu LS, [not published]) show that the combined front and rear bump stop stiffnesses are similar with respect to the left and right side of the vehicle. In addition, no known published material exists stating that a passenger vehicle manufacturer (i.e., excluding specialized race cars) purposely designed a suspension with different left versus right side spring and bump stop stiffnesses. Therefore, the SLX's suspension rates are unusual.

Unfortunately, the maximum roll angles achieved during the roll suspension tests were only about $\pm 4^{\circ}$ (Figure 5.5), while the roll angles in the field tests reached values of $-15^{\circ}$ (right steer) to $21^{\circ}$ (left steer) during TWL. Although the roll stiffness of the vehicle is unknown beyond $\pm 4^{\circ}$ of roll, the important consideration is how the suspension responded leading up to TWL, i.e., at roll angles less than $\pm 10^{\circ}$. Both graphs in Figure 5.5 show that beyond $\pm 3^{\circ}$ roll angle, the front suspension is stiffer in CCW body roll. Linear regression analysis shows that the front roll stiffness is 3.1\% greater in CCW versus CW body roll for the nominal load configuration and $12.0 \%$ for the maximum occupancy configuration. Note the roll stiffness becomes nonlinear for roll angles beyond $-3^{\circ}$, especially in the maximum occupancy configuration. It appears that the bump stops or the anti-roll bar begin to have a predominant effect beyond $-3^{\circ}$ of roll. As for the rear, the rear roll stiffness is $3.2 \%$ greater in CCW versus CW body roll for the nominal load configuration and $2.0 \%$ for the maximum occupancy configuration (refer to Figure 5.6). 

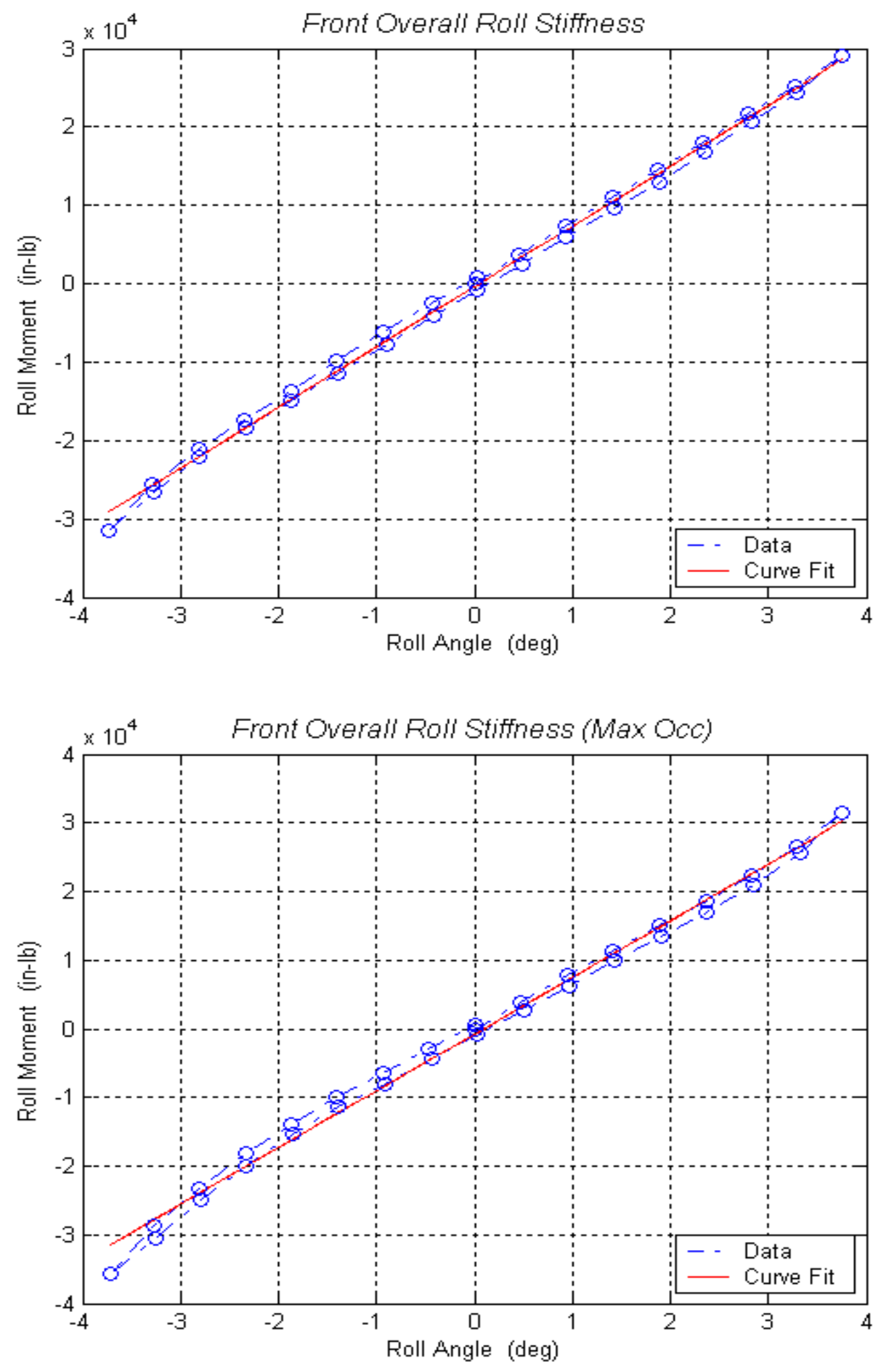

Figure 5.5: SLX Front Overall Roll Stiffness 

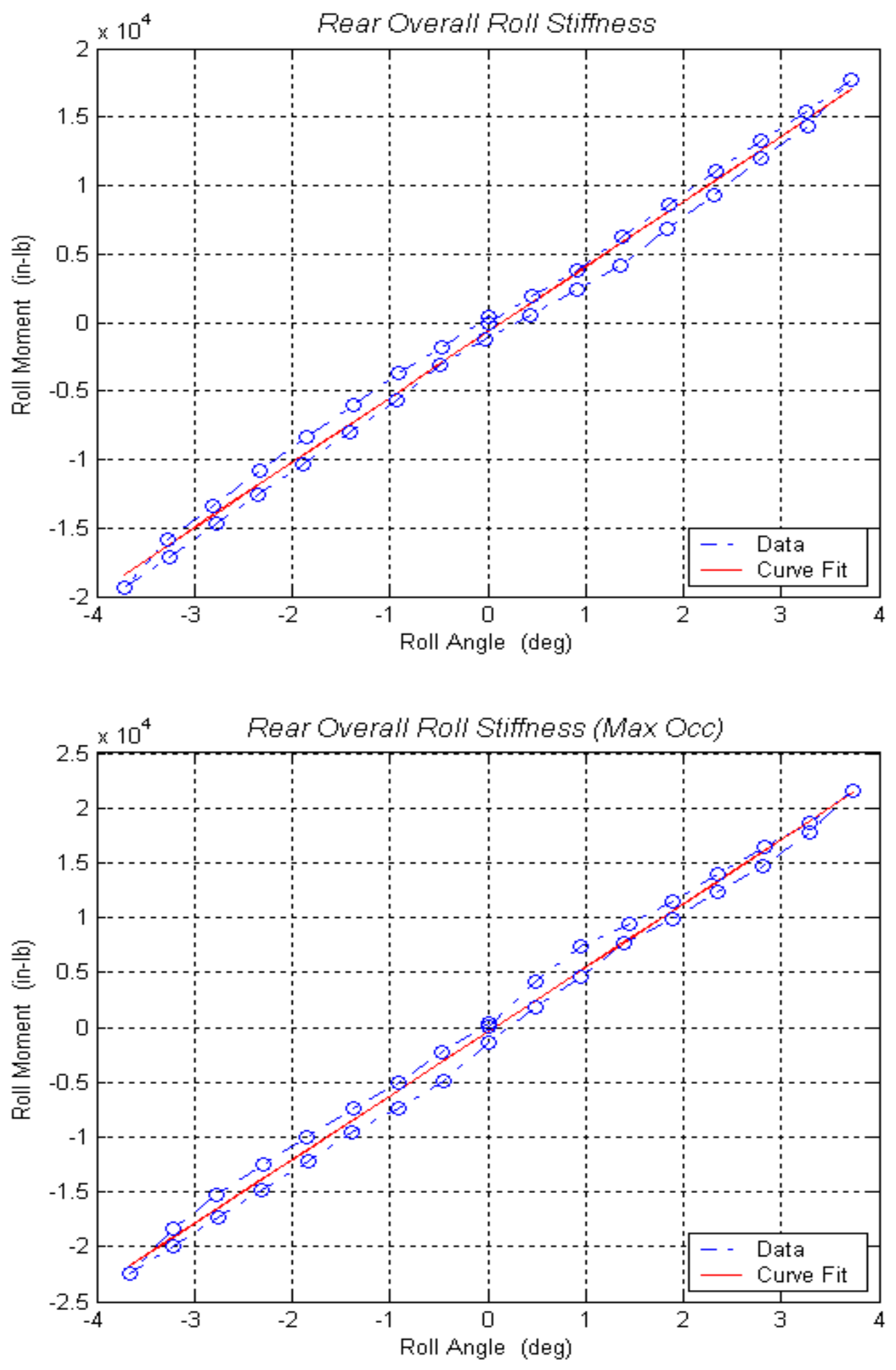

Figure 5.6: SLX Rear Overall Roll Stiffness 
Figure 5.7 presents the front roll steer for both load configurations. The right wheel had less roll steer than the left wheel for both load configurations. The roll steer was in the opposite rotational direction of the wheel steer needed to create the vehicle roll in a driving situation. For instance, negative wheel steer causes the vehicle to steer left, which causes the vehicle body to roll away from the turn (positive roll), which causes positive roll steer. This countersteer phenomenon is called roll understeer. For example, based on the nominal load test, during a right steer maneuver (roll CCW (-)), both tires exhibited countersteer $\left(-0.20^{\circ}\right.$ for the right wheel and $-0.24^{\circ}$ for the left wheel at about $4^{\circ}$ of roll). During a left steer maneuver (roll CW $(+)$ ), the countersteer magnitudes were $0.14^{\circ}$ for the right wheel and $0.25^{\circ}$ for the left wheel at about $4^{\circ}$ of body roll. Note the roll steer on the heavier loaded tire during a turn that produces $\pm 4^{\circ}$ of roll: during a right steer, the left wheel has $-0.20^{\circ}$ of roll steer, and during a left steer, the right wheel has $0.14^{\circ}$. This phenomenon also occurs in the maximum occupancy configuration, although to a lesser extent.

The differences in the roll steer of the heavily loaded tire indicate that there is less roll countersteer in a left steer maneuver, and thus given identical inputs, a left steer maneuver should produce more lateral acceleration. However, the roll steer magnitudes are very small (less than $\pm 0.3^{\circ}$ for the roll angles tested) for both load configurations, and thus roll steer has little effect on the amount of lateral force generated by the tires.

In both tests, the vehicle was first rolled $\mathrm{CW}$ to $4^{\circ}$ and then $\mathrm{CCW}$ to $-4^{\circ}$ and finally $\mathrm{CW}$ to $0^{\circ}$. Note in the maximum occupancy test that during the CCW roll, the wheels appeared to "stick" around $-0.08^{\circ}$ steer angle, and upon the final CW roll motion, they rotated to $0.07^{\circ}$ steer angle. Ideally, the steer angle should have returned to $0^{\circ}$ once 
the roll angle reached $0^{\circ}$. The maximum occupancy roll test was performed twice to verify this response, and the wheels "stuck" even more in the second test.

Unlike the front roll tests, the rear roll tests showed negligible differences for the roll steer between the left and right wheels in both roll directions, and therefore the rear roll steer graphs are not shown. The negligible differences in roll steer are typical for a solid axle rear suspension, where the entire axle yaws slightly during roll causing both wheels to rotate in the same manner. 
Front Roll Steer
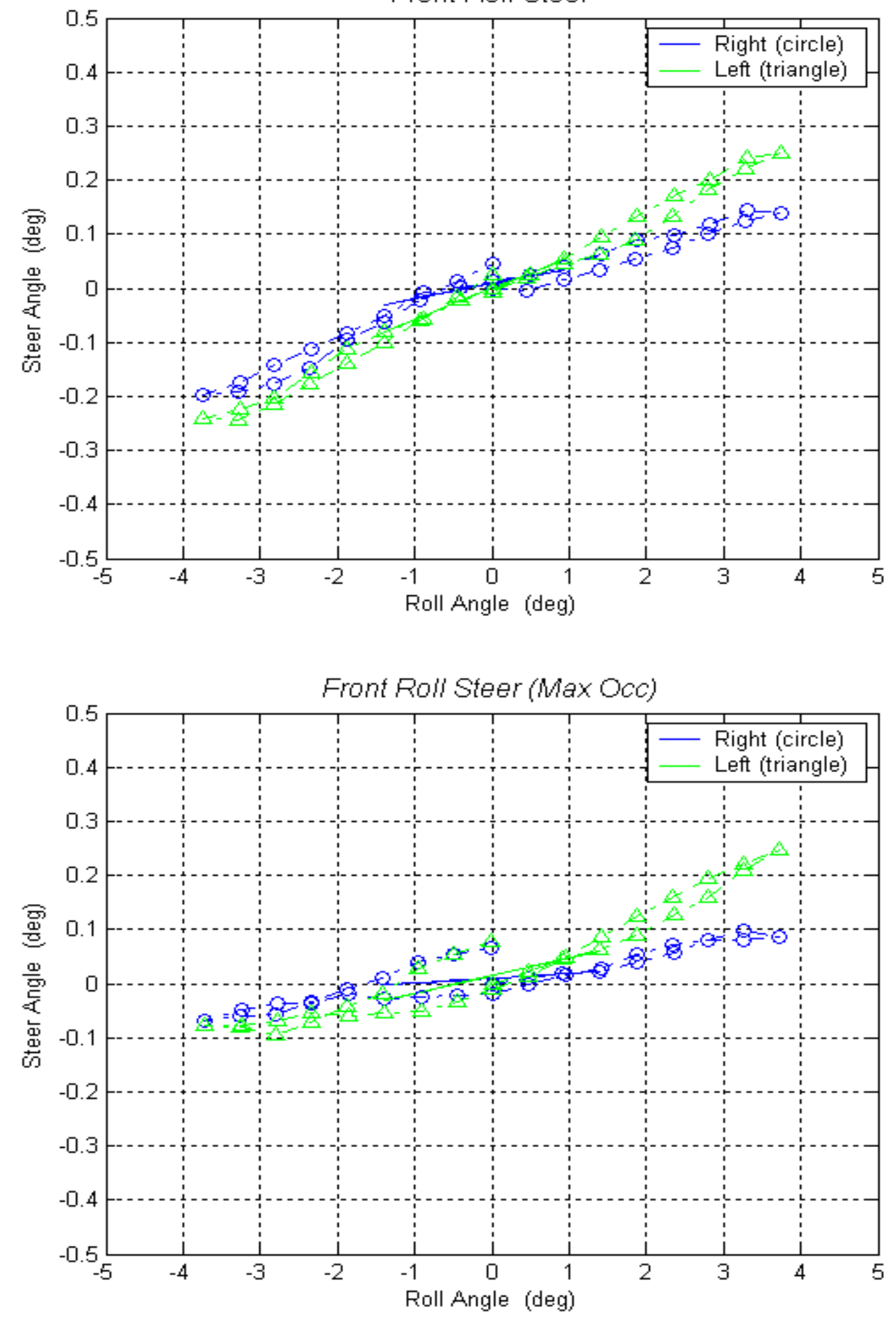

Figure 5.7: SLX Front Roll Steer 
The SLX was tested for front and rear lateral force compliance. Compliances result from flexibility and deflection of the suspension components (mainly rubber bushings). These deflections cause some additional wheel steer and camber angle, as well as changing the effective track width of the vehicle. The front right and left wheel lateral force steer compliances are shown in Figure 5.8.

As the front left wheel is pulled to the right (i.e., in a right turn, top graph in Figure 5.8), the left wheel steers toward the right, and vice versa for the right front wheel (bottom graph in Figure 5.8). The magnitude of the steer angle is negligible though $\left(0.10^{\circ}\right.$ for left wheel and $-0.01^{\circ}$ for right wheel, both under $600 \mathrm{lb}$ of lateral force). The rear lateral force compliance tests showed negligible differences between the left and right side wheels, and consequently, the graphs for these tests are not shown. 

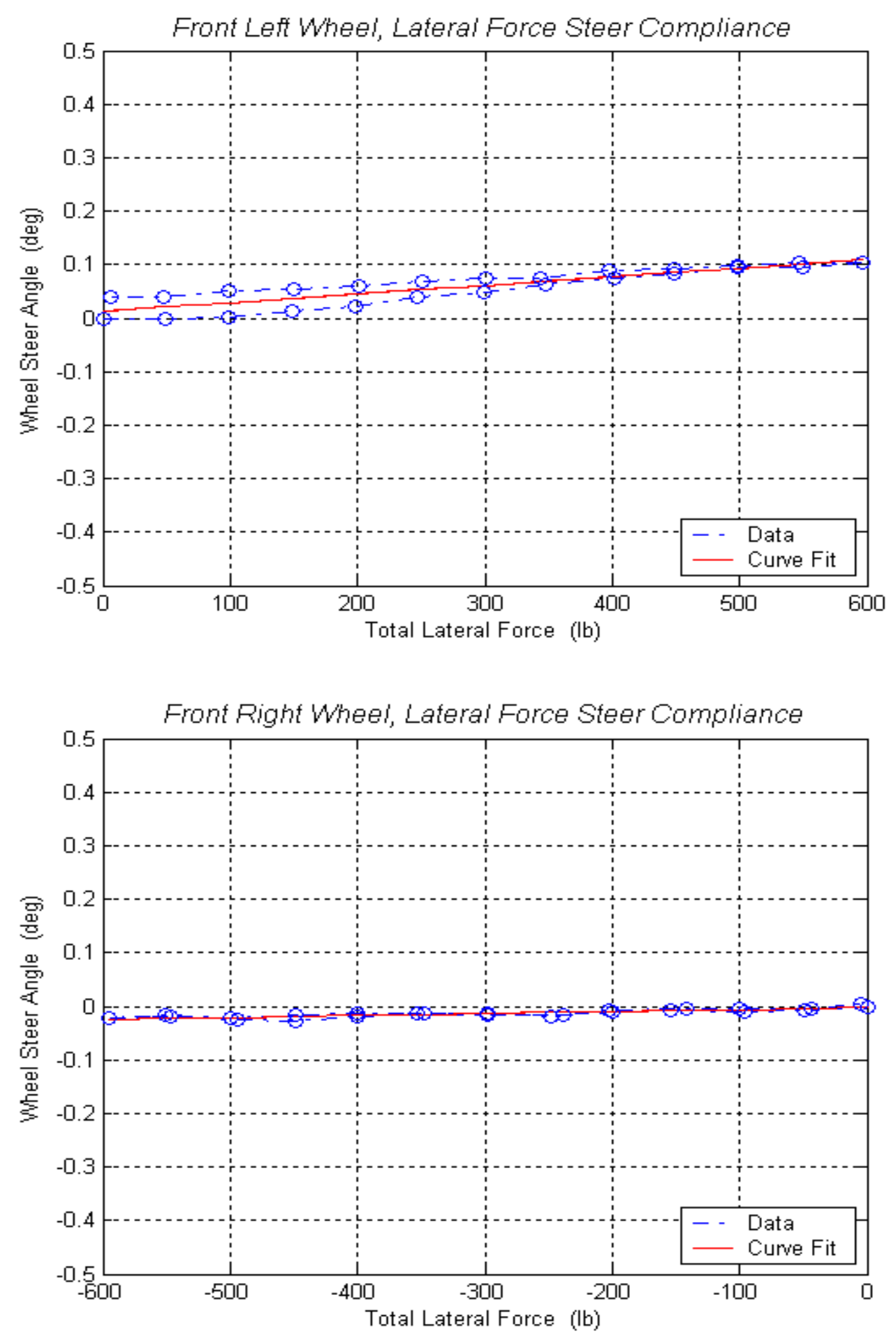

Figure 5.8: SLX Front Wheel Lateral Force Steer Compliance 
The results of the nominal load configuration front steering compliance tests are shown in Figure 5.9. The maximum occupancy configuration tests had similar results to the nominal load configuration tests and therefore are not shown. As expected for a solid rear axle suspension, the rear steering compliance showed negligible differences between CW and CCW applied moments, and therefore, no graphs are presented for the rear steering compliance.

The steering rack attachment and the suspension link attachments will determine how much compliance steer there is in each case. Bent steering links (for packaging reasons) are more compliant than straight links, because a bent link in compression or tension will bend more easily. Also, longer links are more compliant than shorter links. Furthermore, during vehicle roll, the wheels move in opposite directions (one up and the other down) and the steering rack has a side force trying to move it to either the left or right [22].

A significant amount of compliance would mean that a given handwheel angle would produce less wheel steer than the steering ratio expectation. However, it is difficult to quantify the effect of compliance steer, since the aligning moment of the tire during a maneuver is unknown for the SLX. Although, published aligning moment data show that magnitudes can reach up to $200 \mathrm{ft}-\mathrm{lb}$ for a heavy loaded tire with moderate slip angles $\left(4^{\circ}\right.$ to $\left.8^{\circ}\right)$ [13]. This range of aligning moment was also observed in the severe simulation runs that will be presented in Chapter 6 .

The SLX's tie rods were connected to the steering arm in front of the wheel center (forward-steer configuration). The track rod and both tie rods were straight (no bends). The pitman arm was on the driver side, and consequently, the left wheel had less 
compliance than the right wheel (fewer links connecting the left wheel to the pitman arm).

Based on the nominal load configuration front steering compliance tests, during a right turn, the right front wheel's compliance is $0.00549 \mathrm{deg} /(\mathrm{ft}-\mathrm{lb})$ and the left wheel's compliance is $0.00491 \mathrm{deg} /(\mathrm{ft}-\mathrm{lb})$; therefore the average is $0.00520 \mathrm{deg} /(\mathrm{ft}-\mathrm{lb})$. During a left turn, the right front wheel's compliance is $0.00452 \mathrm{deg} /(\mathrm{ft}-\mathrm{lb})$, and the left wheel's compliance is $0.00413 \mathrm{deg} /(\mathrm{ft}-\mathrm{lb})$; therefore the average is $0.00433 \mathrm{deg} /(\mathrm{ft}-\mathrm{lb})$. Thus, there is $20.1 \%$ more compliance in a right turn. Given the same handwheel angle magnitude, a left steer maneuver will produce more wheel steer than a right steer maneuver. However, given that the aligning moment is estimated to be less than $200 \mathrm{ft}$ $\mathrm{lb}$, the maximum change in steer angle due to steering compliance is $1.04^{\circ}$ for a right turn and $0.87^{\circ}$ for a left turn, which is negligible. 

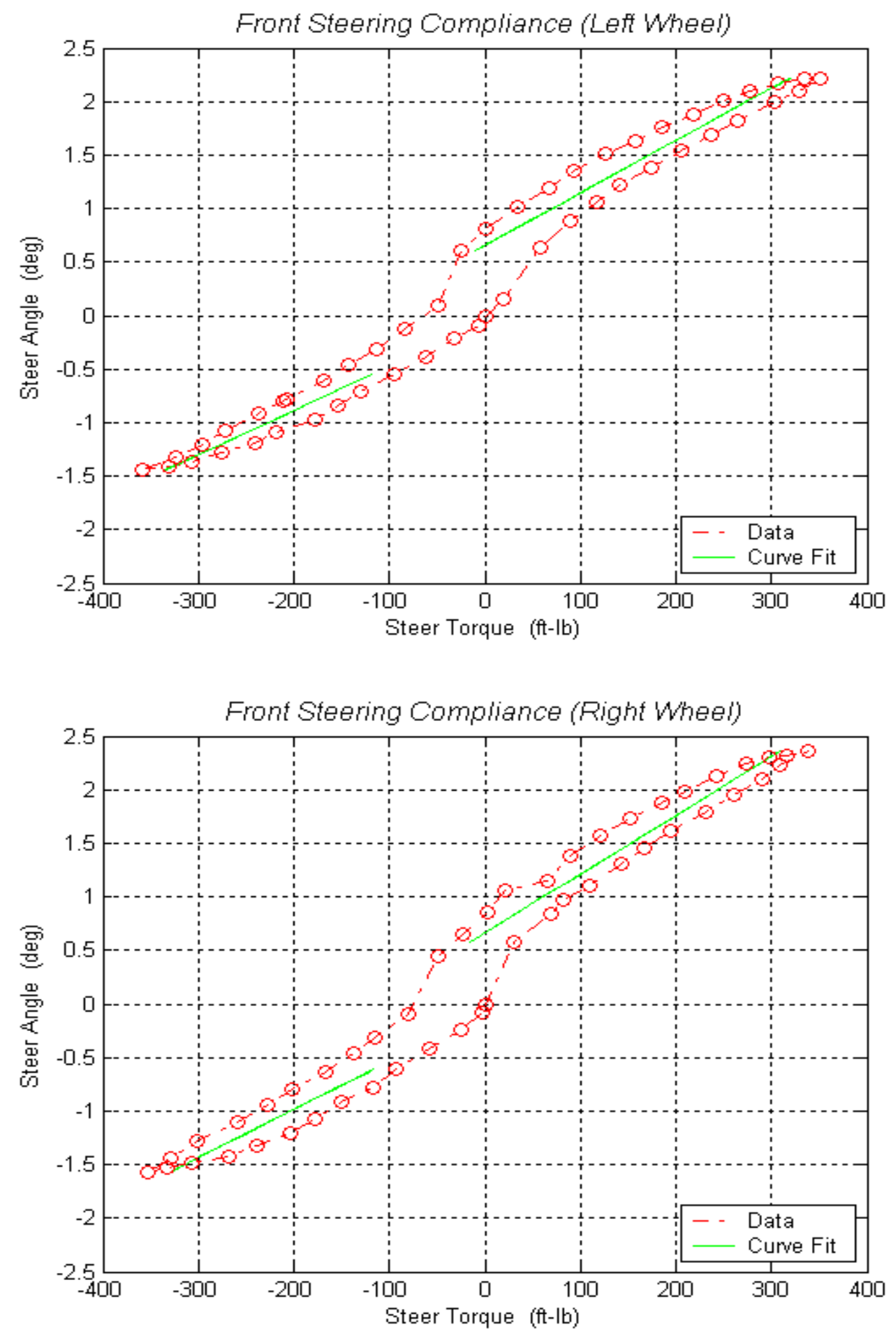

Figure 5.9: SLX Front Steering Compliance 
The results of the steering ratio tests are shown in Figure 5.10. The typical handwheel (steering) angle used in everyday driving is less than $\pm 360^{\circ}$, and the maximum used for the SLX’s rollover tests was $\pm 386^{\circ}$ (J-turn). Consequently, the steering ratio was computed based on the linear fit between $\pm 360^{\circ}$ handwheel angle. Still, the handwheel angle was tested from lock-to-lock $\left(732^{\circ}\right.$ and $\left.-736^{\circ}\right)$ to reflect the performance during low speed maneuvers (e.g. parallel parking).

Although it is not apparent in Figure 5.10, it is common for the steering ratio plots to contain loops, which is a result of hysteresis and means that there is some compliance and/or loss motion (slop) in the steering system.

There was negligible difference between the left and right wheel steering ratios, $19.76 \mathrm{deg} / \mathrm{deg}$ and $19.85 \mathrm{deg} / \mathrm{deg}$, respectively. The steering ratio steering right is 20.22 deg/deg and steering left is $19.57 \mathrm{deg} / \mathrm{deg}$ (taken as the average of both wheel steer angles versus handwheel angle, where $0^{\circ}$ to $360^{\circ}$ is right steer and $0^{\circ}$ to $-360^{\circ}$ is left steer). Furthermore, the wheel steer angle of the heavier loaded outside tire at $\pm 360^{\circ}$ handwheel angle is $17.38^{\circ}$ (right steer, left front tire) and $-17.63^{\circ}$ (left steer, right front tire). All of these values indicate that there is negligible difference in the handwheel angle versus wheel steer angle between a left steer and right steer maneuver. 

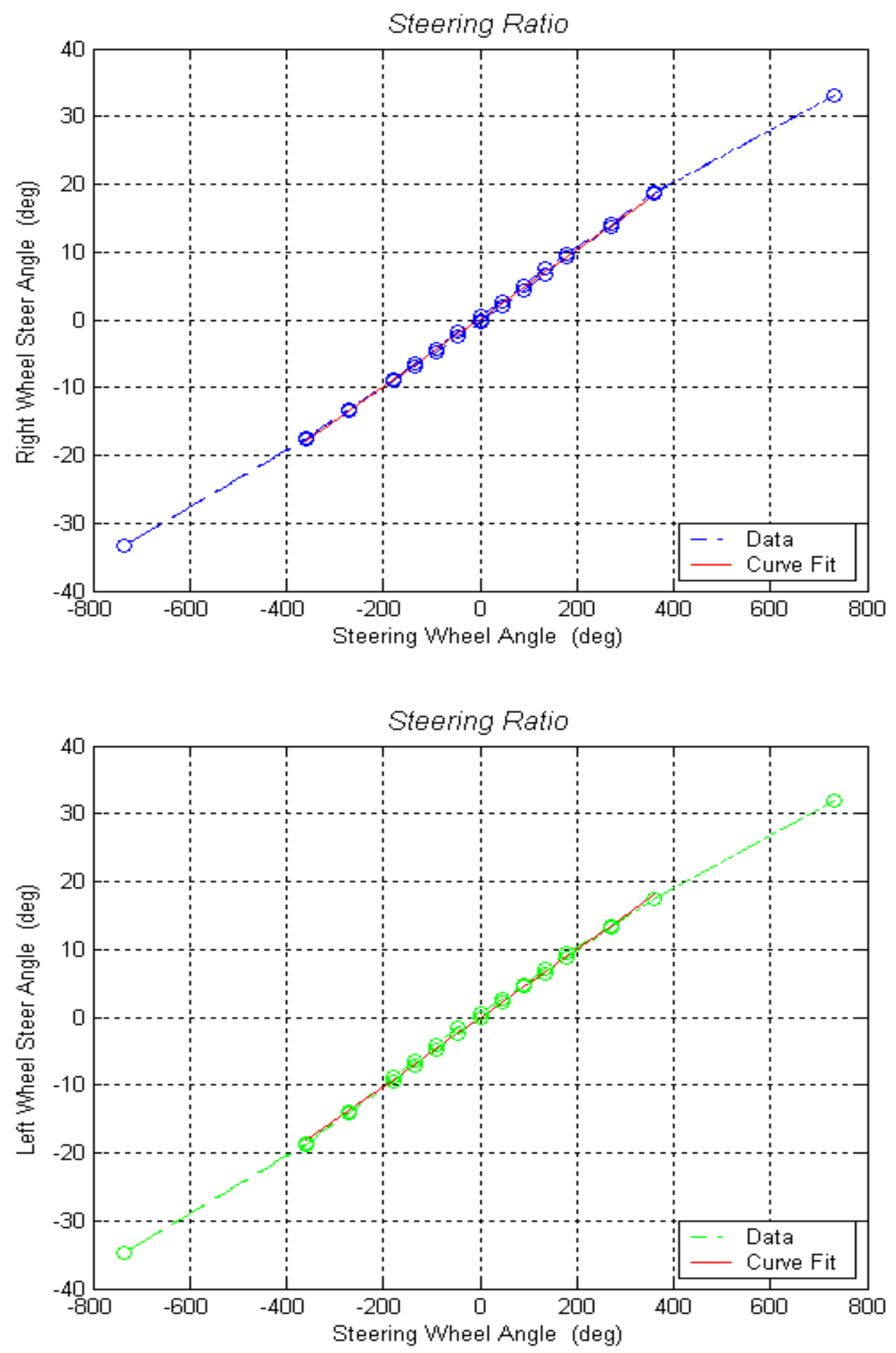

Figure 5.10: SLX Steering Ratio 


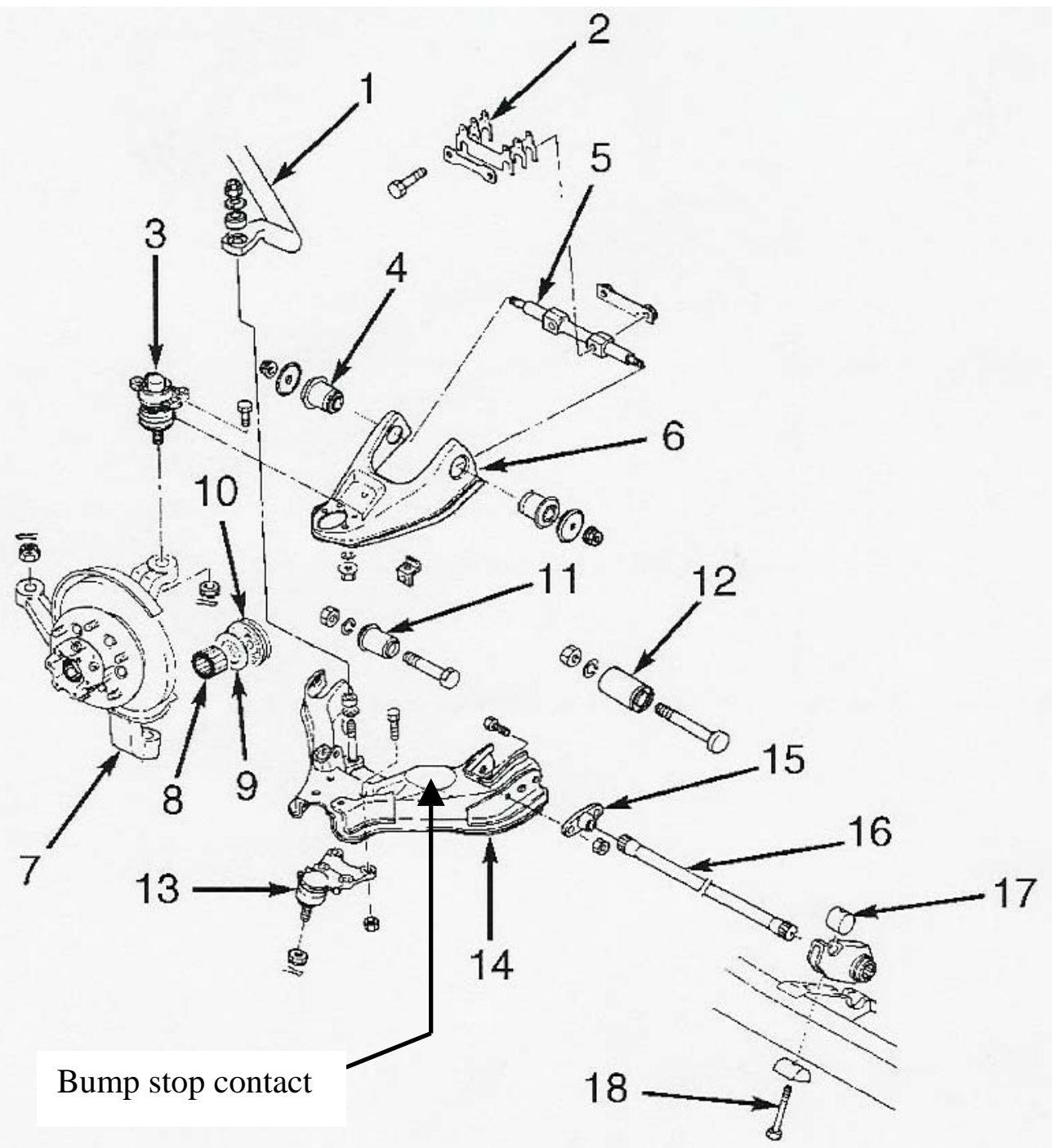

1. Stabilizer Shaft

10. Oil Seal

2. Caster \& Camber Shims

3. Upper Ball Joint

4. Upper Control Arm Bushing

11. Lower Control Arm Bushing

5. Pivot Shaft

6. Upper Control Arm

12. Bushing

13. Lower Ball Joint

14. Lower Control Arm

15. Torsion Bar Arm

7. Steering Knuckle

16. Torsion Bar

8. Needle Bearing

17. Height Control Arm.

9. Washer

Figure 5.11: SLX Front Suspension [26]

Typical Torsion Bar Suspension 


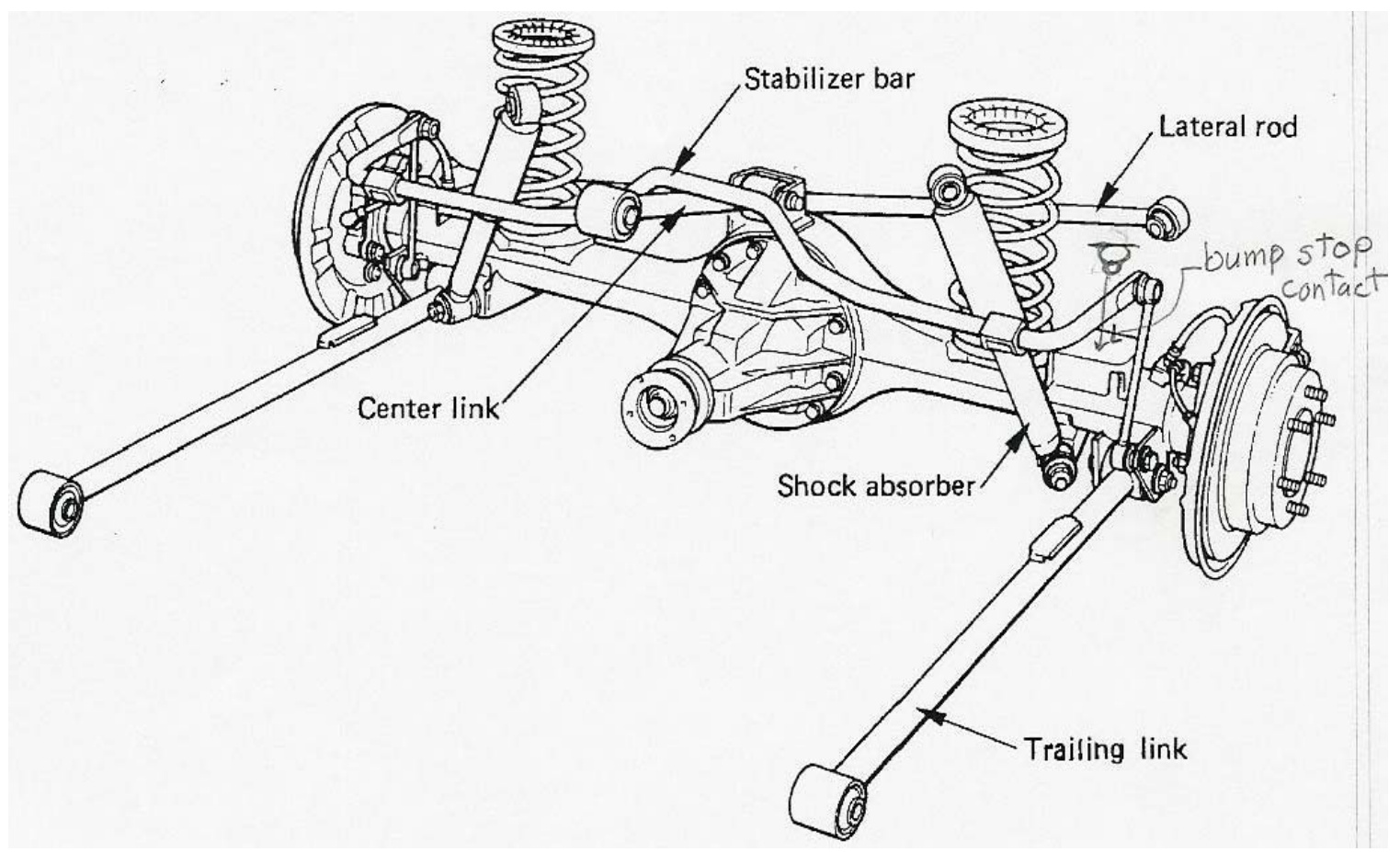

Figure 5.12: SLX Rear Suspension [26] 


\subsubsection{Montero tests}

Similar to Table 5.1 for the SLX, Table 5.3 lists the tests performed on the Montero (DNP denotes “did not perform” test).

\begin{tabular}{|c|c|c|c|}
\hline \multirow{2}{*}{ Test } & Side & \multicolumn{2}{|c|}{ Load Configuration } \\
\cline { 2 - 4 } & $\mathrm{L} / \mathrm{R}$ & Nominal & Max Occ \\
\hline Front Bounce & & $\mathrm{x}$ & $\mathrm{DNP}$ \\
\hline Rear Bounce & & $\mathrm{x}$ & $\mathrm{DNP}$ \\
\hline Front Roll & & $\mathrm{x}$ & $\mathrm{x}$ \\
\hline Rear Roll & & $\mathrm{x}$ & $\mathrm{x}$ \\
\hline Front Lateral Compliance & $\mathrm{L}$ & $\mathrm{x}$ & $\mathrm{x}$ \\
\hline Front Lateral Compliance & $\mathrm{R}$ & $\mathrm{x}$ & $\mathrm{DNP}$ \\
\hline Rear Lateral Compliance & $\mathrm{L}$ & $\mathrm{x}$ & $\mathrm{x}$ \\
\hline Rear Lateral Compliance & $\mathrm{R}$ & $\mathrm{x}$ & $\mathrm{DNP}$ \\
\hline Out-of-Phase Longitudinal Compliance & $\mathrm{L}$ & $\mathrm{x}$ & $\mathrm{DNP}$ \\
\hline Out-of-Phase Longitudinal Compliance & $\mathrm{R}$ & $\mathrm{x}$ & $\mathrm{DNP}$ \\
\hline Front Steering Compliance & $\mathrm{L}$ & $\mathrm{x}$ & $\mathrm{x}$ \\
\hline Front Steering Compliance & $\mathrm{R}$ & $\mathrm{x}$ & $\mathrm{DNP}$ \\
\hline Rear Steering Compliance & $\mathrm{L}$ & $\mathrm{x}$ & $\mathrm{x}$ \\
\hline Rear Steering Compliance & $\mathrm{R}$ & $\mathrm{x}$ & $\mathrm{DNP}$ \\
\hline Steering Ratio Test & & $\mathrm{x}$ & $\mathrm{DNP}$ \\
\hline
\end{tabular}

Table 5.3: Montero Suspension and Steering Tests 


\begin{tabular}{|c|c|c|}
\hline \multicolumn{3}{|c|}{ Montero Front Bounce Test Results } \\
\hline & Front Right & Front Left \\
\hline $\begin{array}{l}\text { Front Ride Rate } \\
\text { Vertical Force vs. Suspension Deflection }\end{array}$ & $159.2 \mathrm{lb} / \mathrm{in}$ & $153.8 \mathrm{lb} / \mathrm{in}$ \\
\hline $\begin{array}{l}\text { Front Bump Stop Stiffness* } \\
\text { Vertical Force vs. Suspension Deflection }\end{array}$ & $\begin{array}{l}1430 \mathrm{lb} / \mathrm{in} \\
5048 \mathrm{lb} / \mathrm{in}\end{array}$ & $\begin{array}{l}1403 \mathrm{lb} / \mathrm{in} \\
4216 \mathrm{lb} / \mathrm{in}\end{array}$ \\
\hline $\begin{array}{l}\text { Front Tire Static Stiffness (26 psi) } \\
\text { Vertical Force vs. Tire Deflection }\end{array}$ & 1096 lb/in & 1086 lb/in \\
\hline \multicolumn{3}{|c|}{ *Nonlinear, broken up and linearized for 3.5-4.6" and 4.6"-max suspension compression } \\
\hline \multicolumn{3}{|c|}{ Montero Rear Bounce Test Results } \\
\hline & Rear Right & Rear Left \\
\hline $\begin{array}{l}\text { Rear Ride Rate } \\
\text { Vertical Force vs. Suspension Deflection }\end{array}$ & $216.7 \mathrm{lb} / \mathrm{in}$ & $209.8 \mathrm{lb} / \mathrm{in}$ \\
\hline $\begin{array}{l}\text { Rear Bump Stop Stiffness* } \\
\text { Vertical Force vs. Suspension Deflection }\end{array}$ & $\begin{array}{c}504 \mathrm{lb} / \mathrm{in} \\
1646 \mathrm{lb} / \mathrm{in}\end{array}$ & $\begin{array}{c}540 \mathrm{lb} / \mathrm{in} \\
1645 \mathrm{lb} / \mathrm{in}\end{array}$ \\
\hline $\begin{array}{l}\text { Rear Tire Static Stiffness (35 psi) } \\
\text { Vertical Force vs. Tire Deflection }\end{array}$ & $1369 \mathrm{lb} / \mathrm{in}$ & 1356 lb/in \\
\hline
\end{tabular}

*Nonlinear, broken up and linearized for 2.0-2.6” and 2.6-3.1” suspension compression (continued)

Table 5.4: Montero Suspension Test Summary 
Table 5.4, continued

Montero Front Roll Test Results

First value given is Nominal Load Case and the other value is Maximum Occupancy Case

\begin{tabular}{|l|c|c|}
\hline & Front Right & Front Left \\
\hline Front Roll Steer & -0.123 & -0.166 \\
Wheel Steer Angle vs. Roll Angle & -0.116 & -0.156 \\
\hline Front Roll Camber & 0.972 & 1.011 \\
Wheel Camber Angle vs. Roll Angle & 0.971 & 0.989 \\
\hline \multirow{2}{*}{ Overall Front Roll Rate } & \multicolumn{2}{|c|}{$9336 \mathrm{in}-\mathrm{lb} / \mathrm{deg}$} \\
Roll Moment vs. Roll Angle & \multicolumn{2}{|c|}{$8818 \mathrm{in}-\mathrm{lb} / \mathrm{deg}$} \\
\hline
\end{tabular}

Montero Rear Roll Test Results

First value given is Nominal Load Case and the other value is Maximum Occupancy Case

\begin{tabular}{|l|c|c|}
\hline & Rear Right & Rear Left \\
\hline Rear Roll Steer & -0.016 & -0.019 \\
Wheel Steer Angle vs. Roll Angle & -0.038 & -0.040 \\
\hline Rear Roll Camber & 0.159 & 0.174 \\
Wheel Camber Angle vs. Roll Angle & 0.170 & 0.173 \\
\hline Overall Rear Roll Rate & \multicolumn{2}{|c|}{7835 in-lb/deg } \\
Roll Moment vs. Roll Angle & \multicolumn{2}{|c|}{7679 in-lb/deg } \\
\hline
\end{tabular}

(continued) 
Table 5.4, continued

\section{Montero Front Steering Compliance Test Results}

First value given is Nominal Load Case and the other value is Maximum Occupancy Case

\begin{tabular}{|l|c|c|}
\hline & Front Right & Front Left \\
\hline Front Steering Compliance (deg/(ft-lb)) & 0.00608 & 0.00668 \\
Applied Steering Torque vs. Steer Angle & DNP & 0.00653 \\
\hline
\end{tabular}

\section{Steering Ratio Test Results}

\begin{tabular}{|l|c|c|}
\hline & Right & Left \\
\hline $\begin{array}{l}\text { Steering Ratio (fit between } \pm 360^{\circ} \text { ) } \\
\text { Steering Wheel Angle vs. Front Wheel Steer Angle }\end{array}$ & 18.4 & 18.3 \\
\hline $\begin{array}{l}\text { Ave. Steering Ratio (fit between } \pm 360^{\circ} \text { ) } \\
\text { Average of Right and Left Measured Ratios }\end{array}$ & & 18.3 \\
\hline
\end{tabular}


The results of the front bounce test are shown in Figure 5.13. The linear suspension spring rate was computed based on the suspension deflection from 0.0 to 2.0 inches during the front bounce test. Typically the spring rate is computed from -1.0 to 1.0 inch of suspension deflection, but the Montero’s front axle unloaded quickly such that the front wheels were impending lift off at only 1.0 inch of suspension rebound.

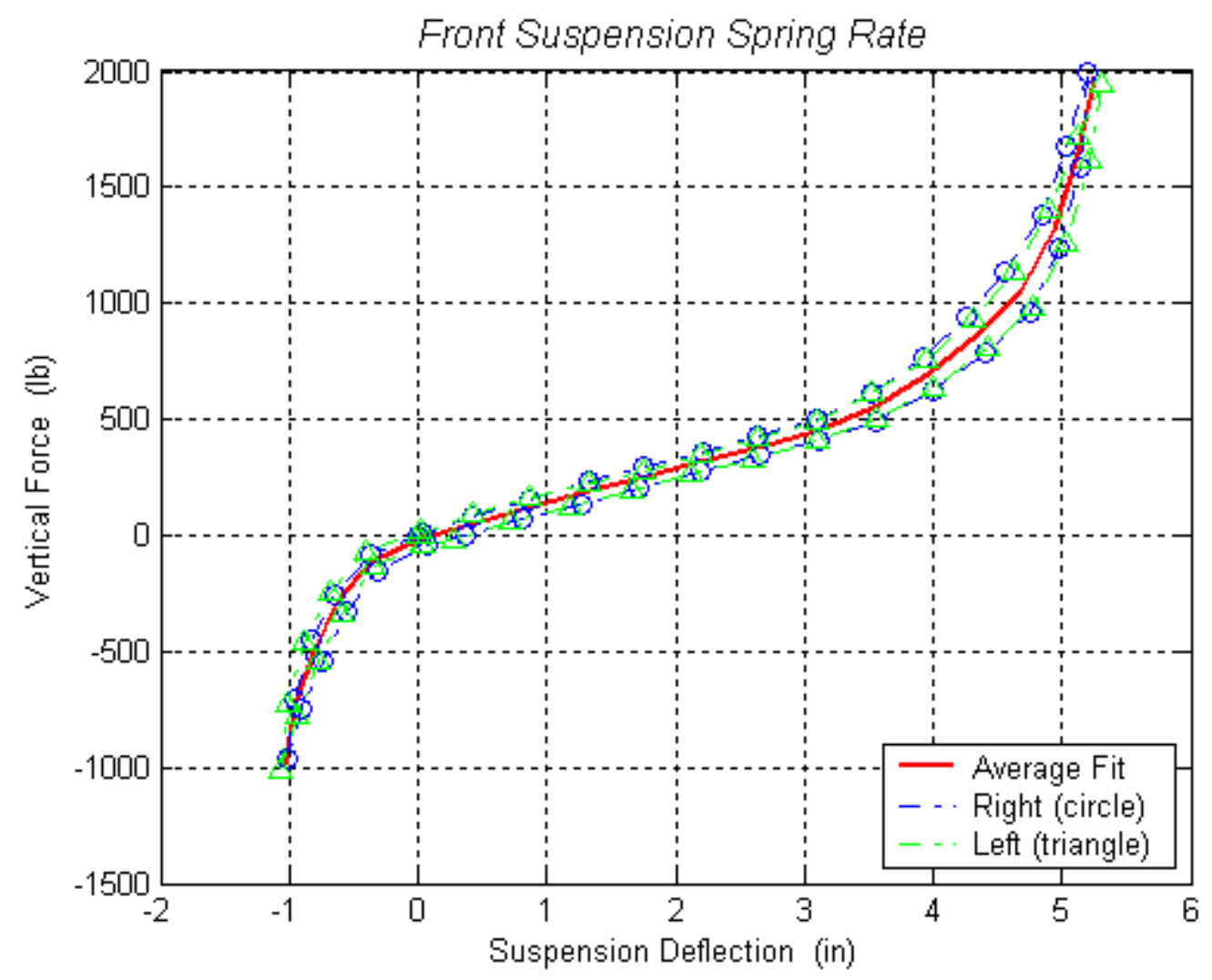

Figure 5.13: Montero Front Suspension Spring Rate 
Beyond five inches of suspension compression, the left side of the suspension is slightly softer than the right side of the suspension. This slight difference is reflected in the upper linearized bump stop values stated in Table 5.4. Note the nonlinear bump stop stiffness (about 3.0 to 5.3 inches of suspension compression) shown in Figure 5.13. A picture of the front bump stops and a diagram of the front suspension (typical torsion bar suspension with a stabilizer bar) are given in Figures 5.22 and 5.21, respectively.

The results of the rear bounce test are shown in Figure 5.14. The linear suspension spring rate was computed based on the suspension deflection from -1.0 to 1.0 inch. Note the nonlinear bump stop stiffness beyond 2.0 inches of suspension compression. Unlike the front suspension, the rear suspension does not have noticeable differences between the left and right side. A picture of the rear bump stops and a diagram of the rear suspension (solid axle with coil springs, stabilizer bar, lateral rod, and trailing links) are given in Figures 5.24 and 5.23, respectively. 


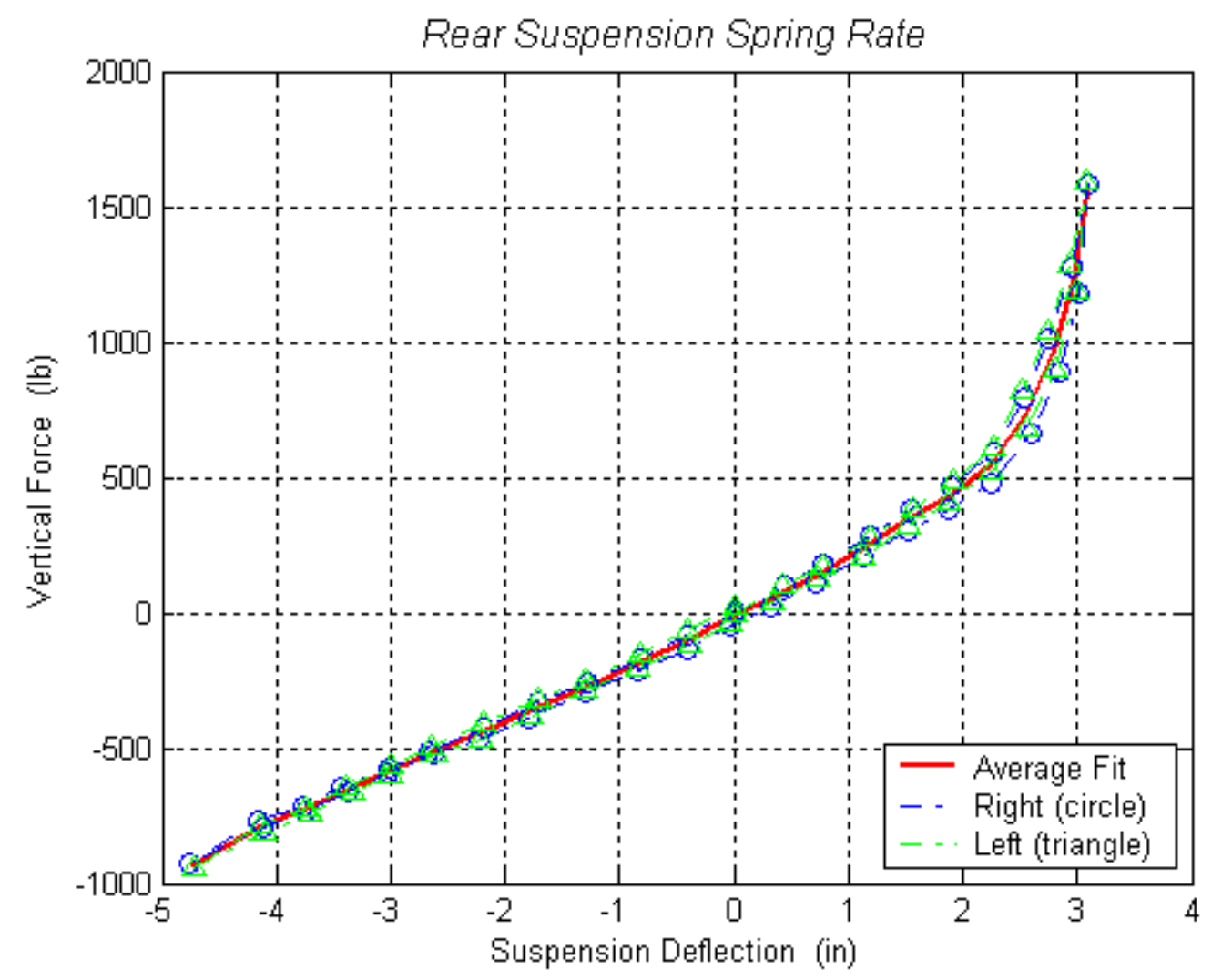

Figure 5.14: Montero Rear Suspension Spring Rate

The differences between the right and left bump stop stiffnesses for the Montero were not as significant as those for the SLX. However, the left side of the suspension is slightly softer than the right side, and therefore the idea proposed earlier that the differences in suspension stiffness contribute to vehicle asymmetrical response is still valid. Furthermore, recall that the SLX experiences TWL at a lower maneuver entrance speed in a left steer versus a right steer J-turn maneuver and its right side suspension is softer than the left side. The same phenomenon applies to the Montero: it experiences TWL at a lower maneuver entrance speed in a right steer J-turn maneuver and its left side suspension is softer. 
Unfortunately due to wheel lift off, the maximum roll angles achieved during the nominal load, front roll stiffness test (Figure 5.15) were $-4.7^{\circ}$ with 6 lbs remaining on the right front tire scale and $5.2^{\circ}$ with $80 \mathrm{lbs}$ remaining on left front tire scale. In the maximum occupancy configuration, the test limits due to wheel lift off were $-4.7^{\circ}$ and $5.7^{\circ}$

Although the roll stiffness of the vehicle is unknown beyond $\pm 5^{\circ}$, the important consideration is how the suspension responded leading up to TWL, i.e., at roll angles less than $\pm 10^{\circ}$. Both nominal load and maximum occupancy Front Roll Stiffness graphs in Figure 5.15 show that the front roll stiffness is slightly stiffer in CW roll (note how the circles begin to deviate from the best fit (solid red) line). Furthermore, linear regression analysis shows that the front roll stiffness is 3.3\% greater in CW versus CCW body roll for the nominal load configuration and $1.6 \%$ for the maximum occupancy configuration. As for the rear, the rear roll stiffness is 3.5\% greater in CW versus CCW body roll for the nominal load configuration and $0.1 \%$ for the maximum occupancy configuration (refer to Figure 5.16). 

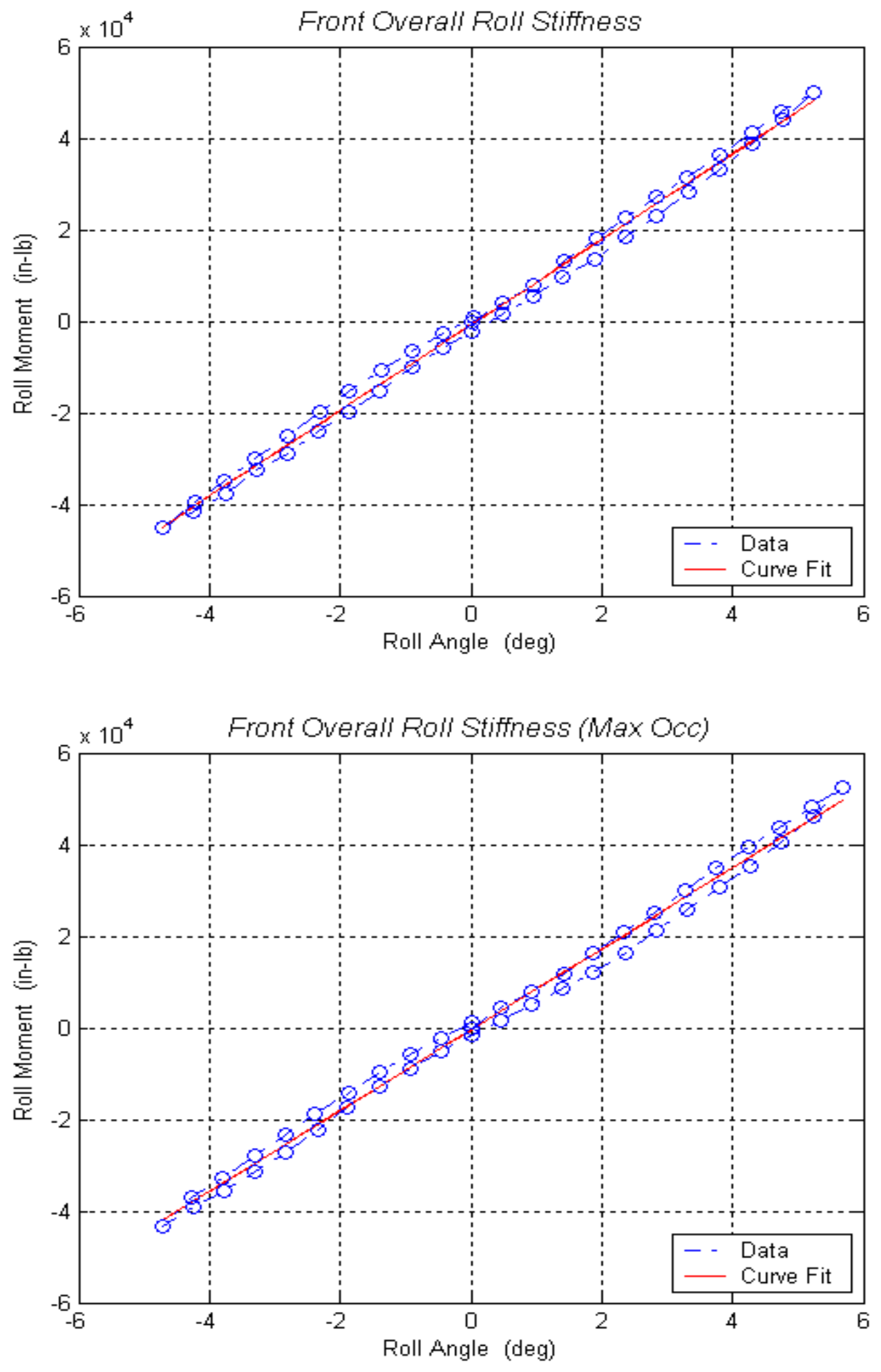

Figure 5.15: Montero Front Overall Roll Stiffness 

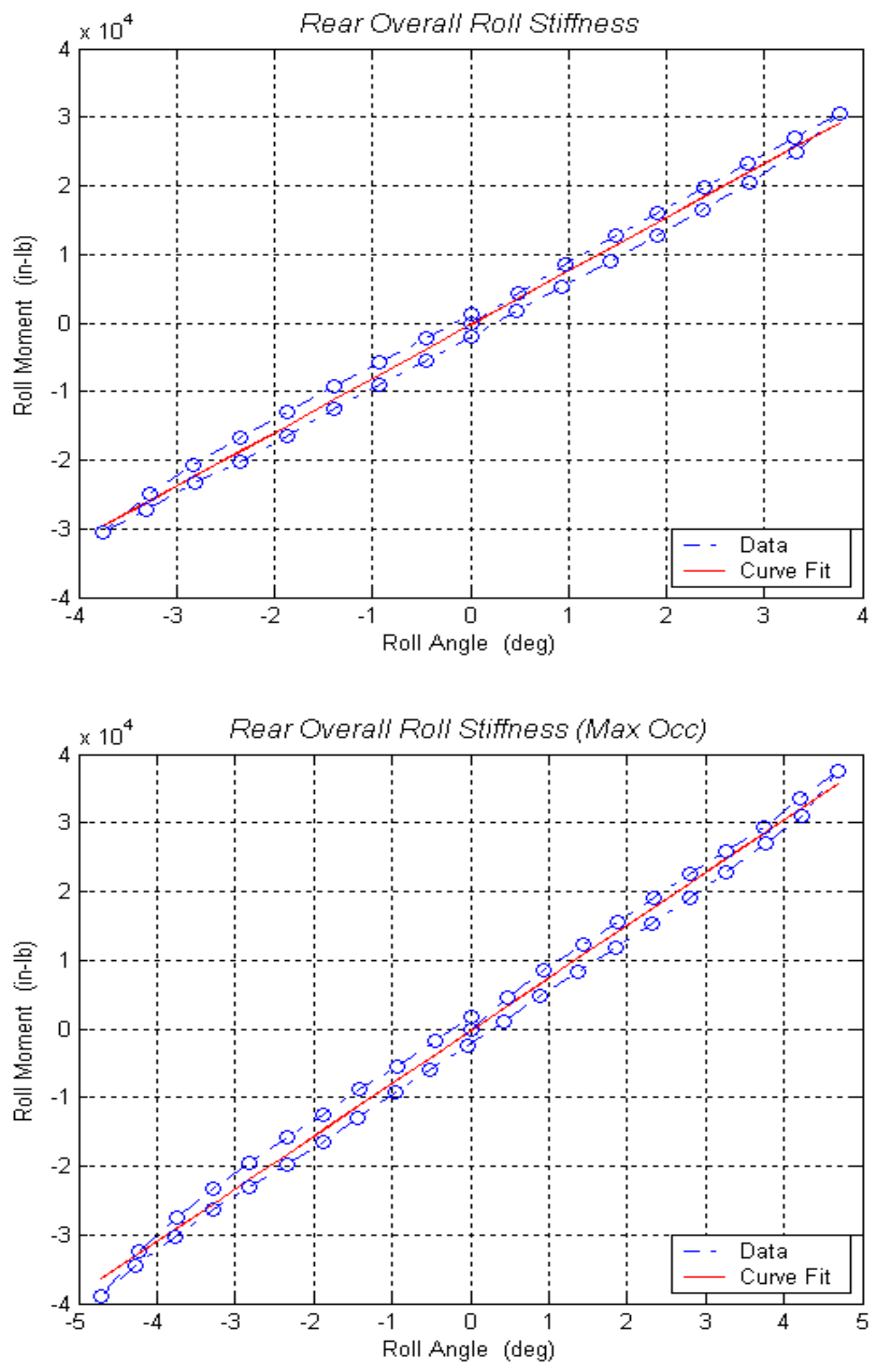

Figure 5.16: Montero Rear Overall Roll Stiffness 
Figure 5.17 presents the front roll steer for both load configurations. The roll steer was in the same rotational direction as the wheel steer needed to create the vehicle roll in a driving situation, i.e., roll oversteer. For example, based on the nominal load test, during a right steer maneuver (roll CCW (-)), both tires exhibited oversteer $\left(0.32^{\circ}\right.$ for the right wheel and $0.40^{\circ}$ for the left wheel at $-4.7^{\circ}$ of roll). During a left steer maneuver (roll to right $(+)$ ), the roll steer magnitudes were $-0.50^{\circ}$ for the right wheel and $-0.80^{\circ}$ for the left wheel at $5.2^{\circ}$ of roll. Note the roll steer on the heavier loaded tire during a turn that produces $\pm 4^{\circ}$ of roll: during a right steer, the left wheel has $0.37^{\circ}$ of roll steer, and during a left steer, the right wheel has $-0.47^{\circ}$. This phenomenon also occurs in the maximum occupancy configuration.

The differences in the roll steer of the heavily loaded tire for both load configurations indicate that there is more roll steer in a left steer maneuver. However, the differences in the roll steer magnitudes are very small (about $0.1^{\circ}$ ) for both load configurations, and thus roll steer has little effect on the differences in lateral force generated by the tires in a left versus right steer maneuver.

Unlike the front roll tests, the rear roll tests showed negligible differences for the roll steer between the left and right wheels in both roll directions, and therefore the rear roll steer graphs are not shown. 
Front Roll Steer
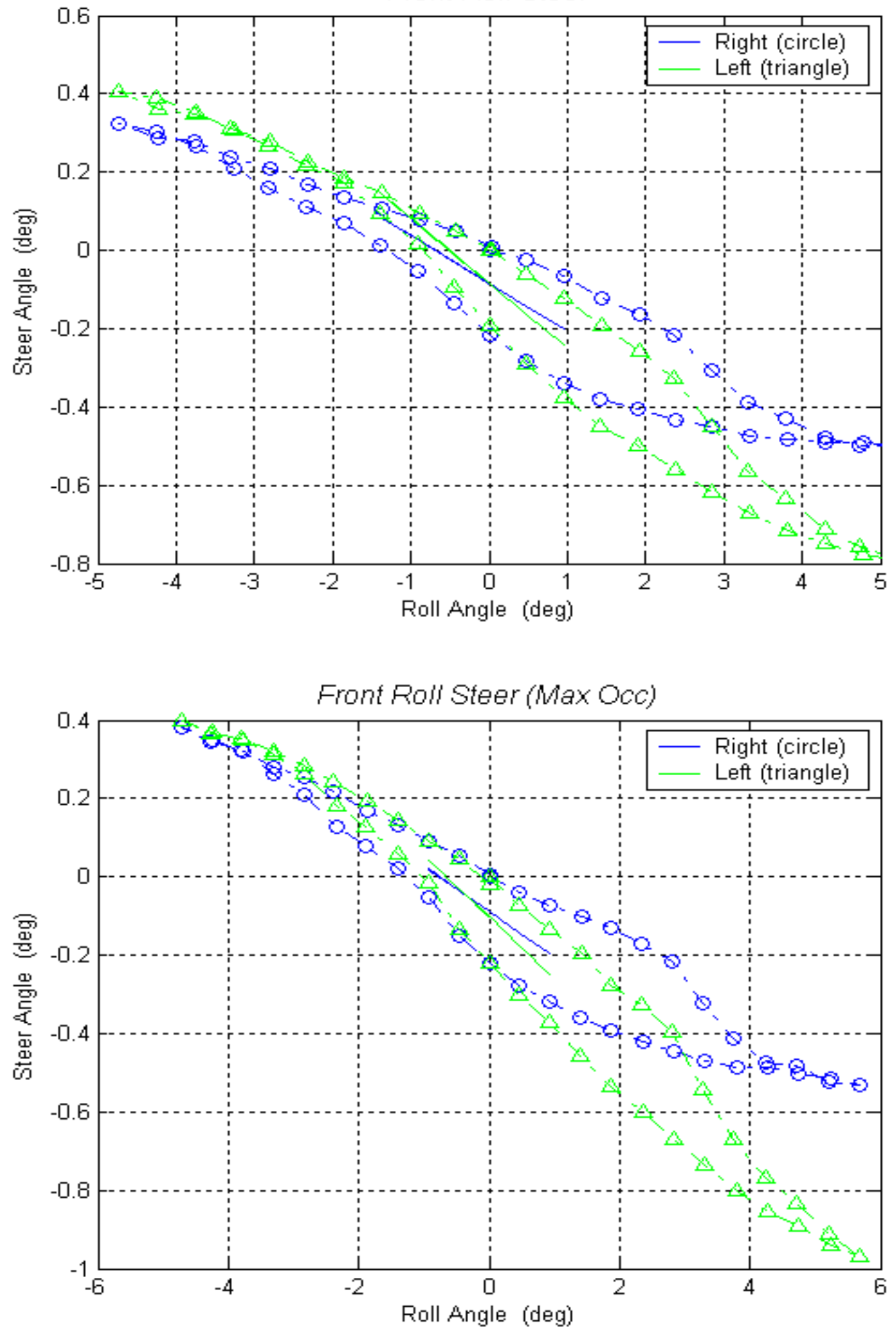

Figure 5.17: Montero Front Roll Steer 
The results of the front lateral force compliance tests are shown in Figure 5.18. As shown in the top graph of Figure 5.18, as the front left wheel is pulled to the right (i.e., in a right turn), the left wheel steers toward the left, and vice versa for the right front wheel (bottom graph of Figure 5.18). The magnitude of the steer angle is negligible though $\left(-0.19^{\circ}\right.$ for left wheel and $0.06^{\circ}$ for right wheel, both under $600 \mathrm{lb}$ of lateral force). The rear lateral force compliance tests showed negligible differences between the left and right wheels, and consequently, the graphs for these tests are not shown. 

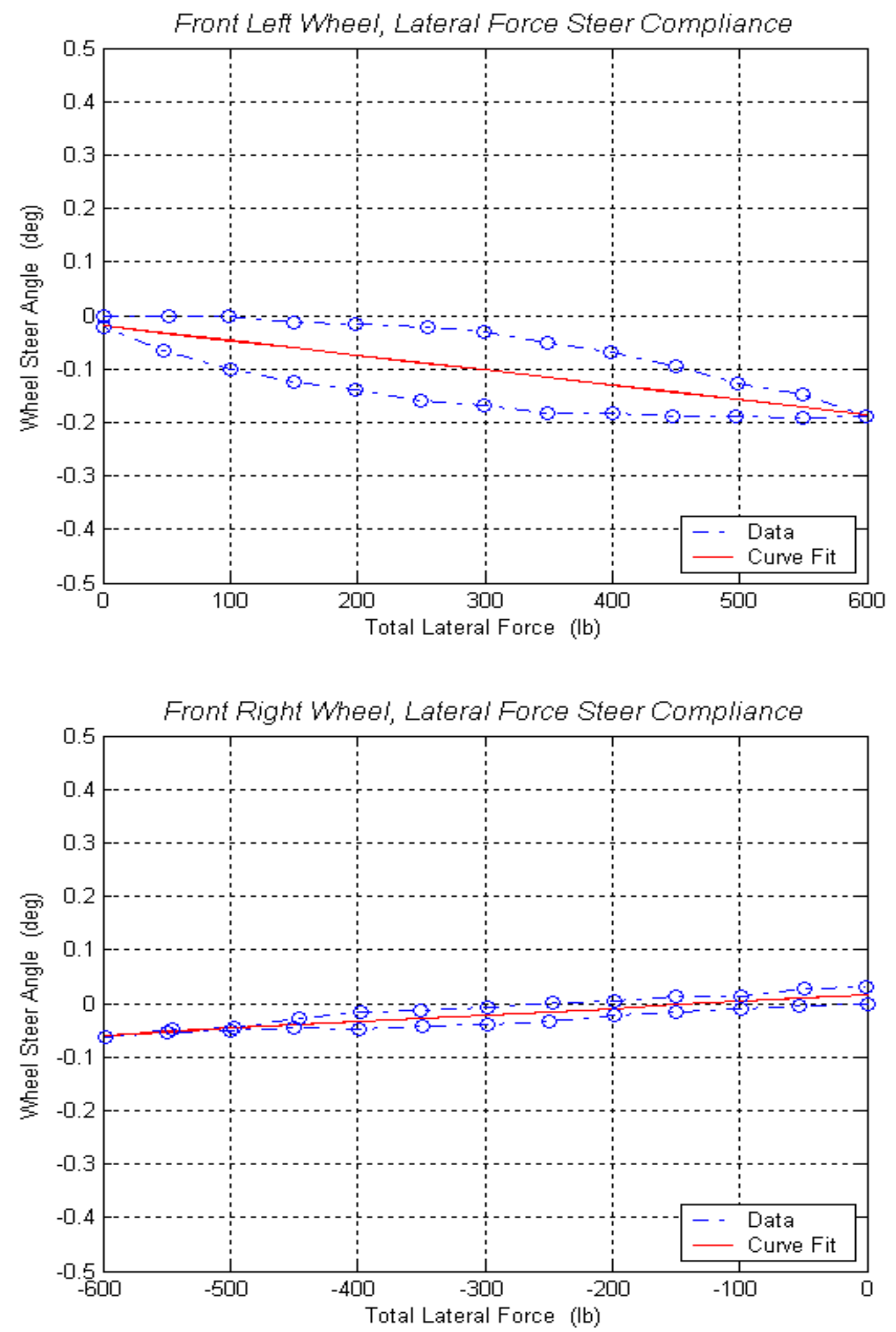

Figure 5.18: Montero Front Wheel Lateral Force Steer Compliance 
The results of the nominal load configuration front steering compliance tests are shown in Figure 5.19. The maximum occupancy configuration tests had similar results to the nominal load configuration and therefore are not shown. As expected for a solid rear axle suspension, the rear steering compliance showed negligible differences between CW and CCW applied moments, and therefore, no graphs are presented for the rear steering compliance.

Based on the nominal load configuration front steering compliance test, during a right turn, the right front wheel's compliance is $0.00690 \mathrm{deg} /(\mathrm{ft}-\mathrm{lb})$ and the left wheel's compliance is $0.00660 \mathrm{deg} /(\mathrm{ft}-\mathrm{lb})$; therefore the average is $0.00675 \mathrm{deg} /(\mathrm{ft}-\mathrm{lb})$. During a left turn, the right front wheel's compliance is $0.00526 \mathrm{deg} /(\mathrm{ft}-\mathrm{lb})$ and the left wheel's compliance is $0.00676 \mathrm{deg} /(\mathrm{ft}-\mathrm{lb})$; therefore the average is $0.00601 \mathrm{deg} /(\mathrm{ft}-\mathrm{lb})$. Thus, there is $12.3 \%$ more compliance in a right turn. Given the same handwheel angle, a left steer maneuver will produce more wheel steer than a right steer maneuver. However, given that the aligning moment is estimated to be less than $200 \mathrm{ft}-\mathrm{lb}$, the maximum change in steer angle due to steering compliance is $1.35^{\circ}$ for a right turn and $1.20^{\circ}$ for a left turn, of which the difference is negligible. 
Front Steering Compliance (Left Wheel)
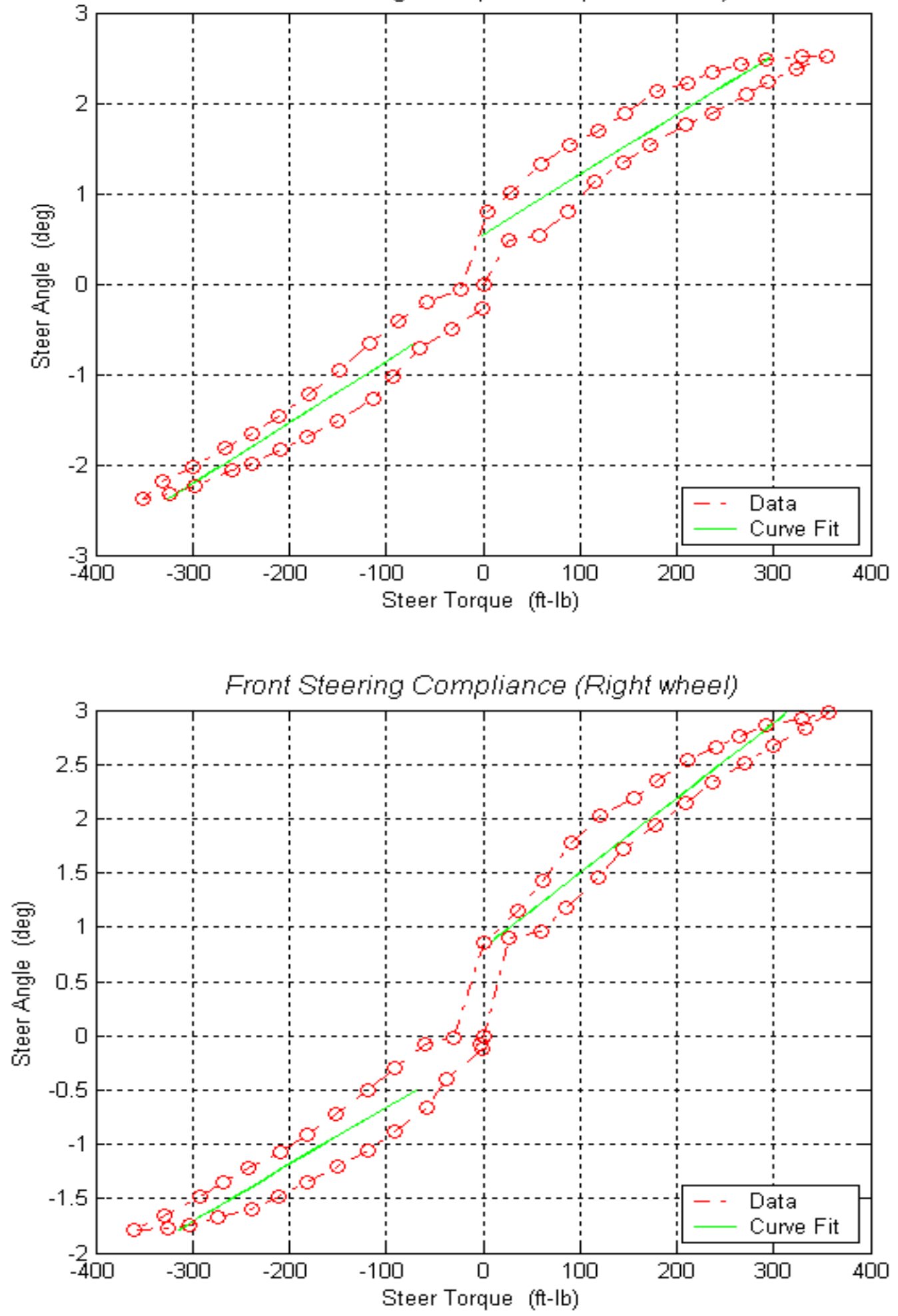

Figure 5.19: Montero Front Steering Compliance 
The results of the steering ratio tests are shown in Figure 5.20. The typical handwheel (steering) angle used in everyday driving is less than $\pm 360^{\circ}$, and the maximum used for the Montero's rollover tests was $\pm 334^{\circ}$ (J-turn). Consequently, the steering ratio was computed based on the linear fit between $\pm 360^{\circ}$ handwheel angle. The handwheel angle was tested from lock-to-lock $\left(609^{\circ}\right.$ and $\left.-640^{\circ}\right)$ to reflect the performance during low speed maneuvers (e.g. parallel parking).

There was negligible difference between the left and right wheel steering ratios, $18.3 \mathrm{deg} / \mathrm{deg}$ and $18.4 \mathrm{deg} / \mathrm{deg}$, respectively. The steering ratio steering right is 18.72 $\mathrm{deg} / \mathrm{deg}$ and steering left is $18.24 \mathrm{deg} / \mathrm{deg}$ (taken as the average of both wheel steer angles versus handwheel angle, where $0^{\circ}$ to $360^{\circ}$ is right steer and $0^{\circ}$ to $-360^{\circ}$ is left steer). Furthermore, the wheel steer angle of the heavier loaded outside tire at $\pm 360^{\circ}$ handwheel angle is $18.75^{\circ}$ (right steer, left front tire) and $-18.25^{\circ}$ (left steer, right front tire). All of these values indicate that there is negligible difference in the handwheel angle versus wheel steer angle between a left steer and right steer maneuver. 

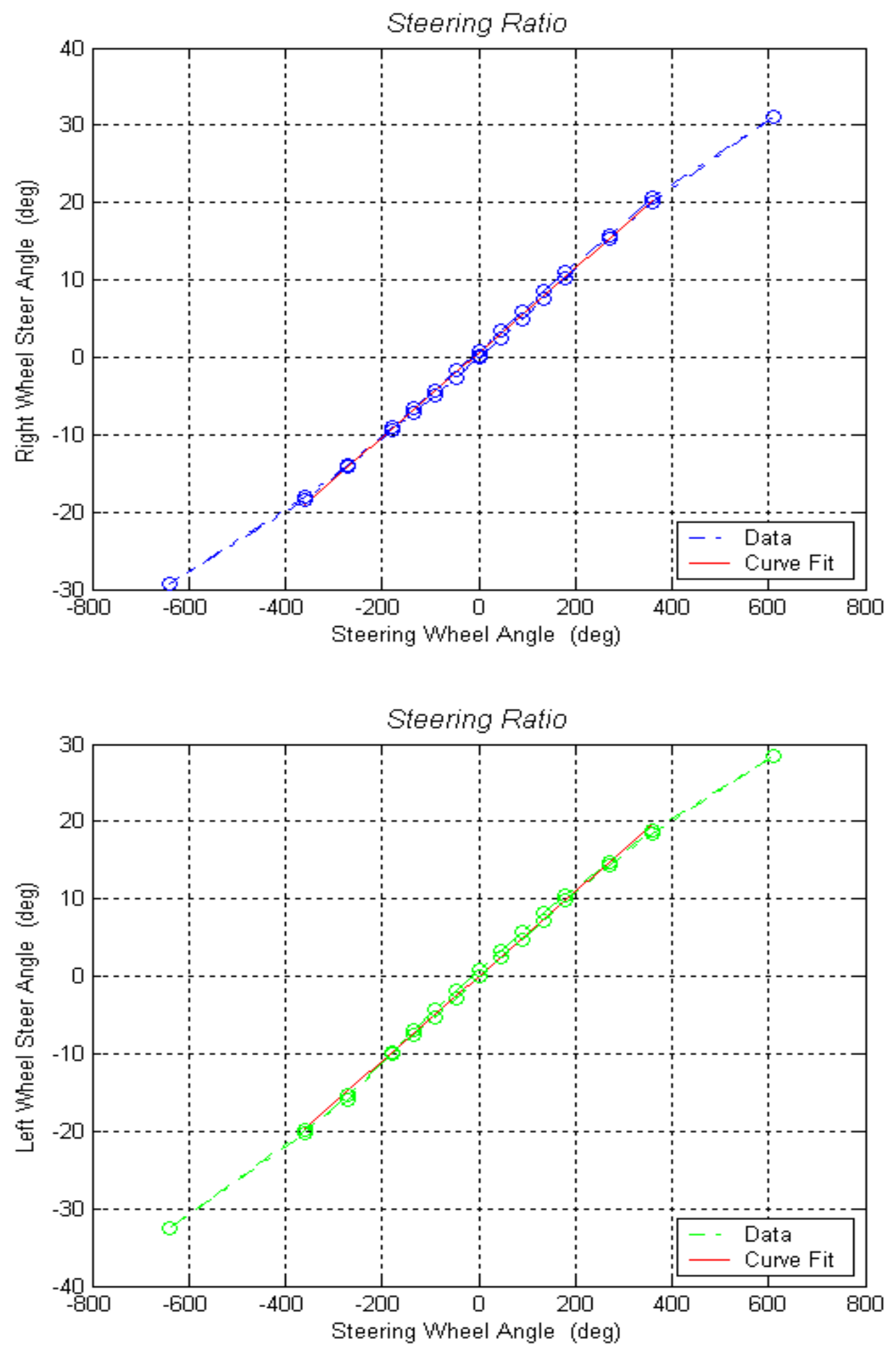

Figure 5.20: Montero Steering Ratio 


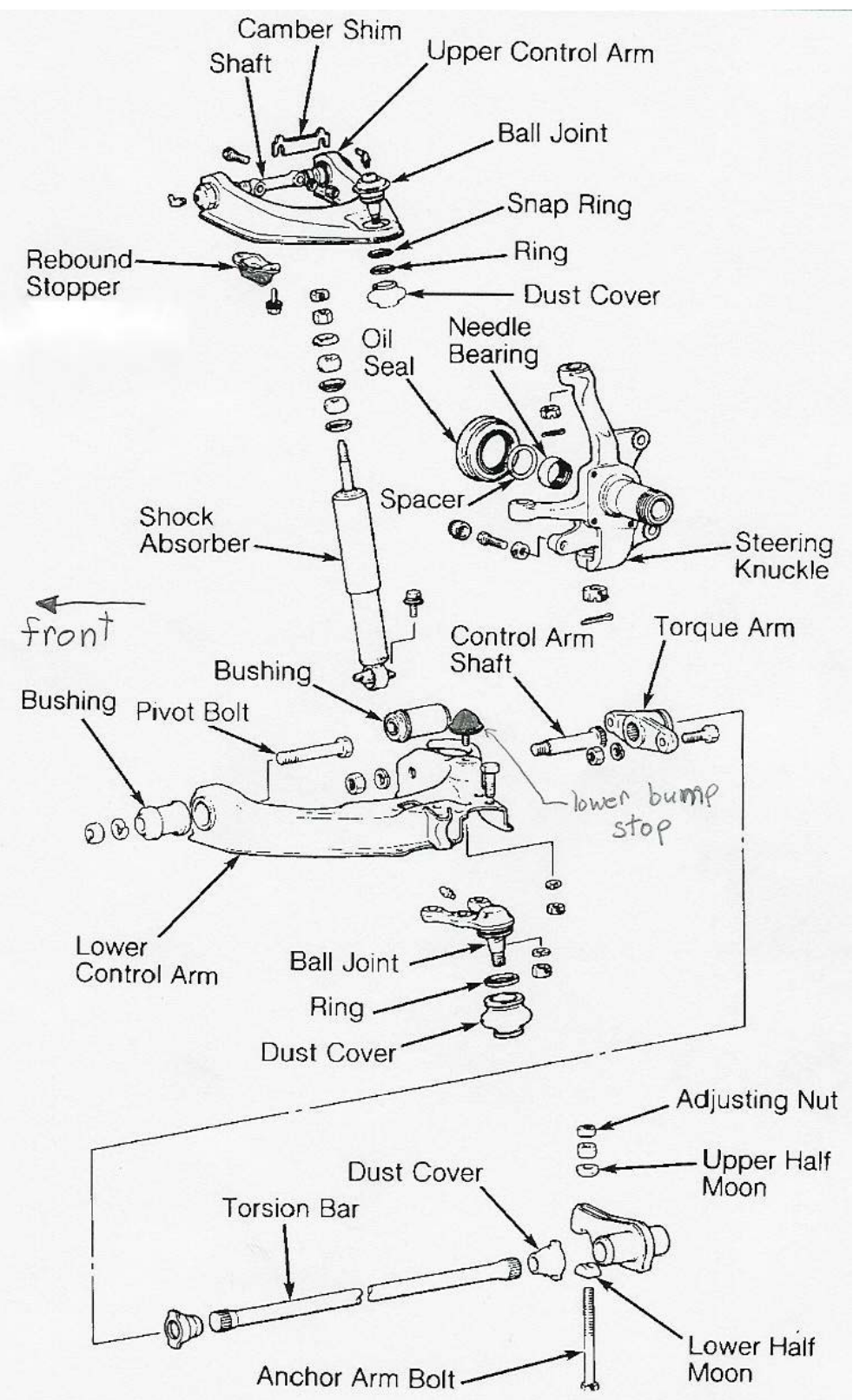

Figure 5.21: Montero Front Suspension [26]

Torsion Bar Suspension 


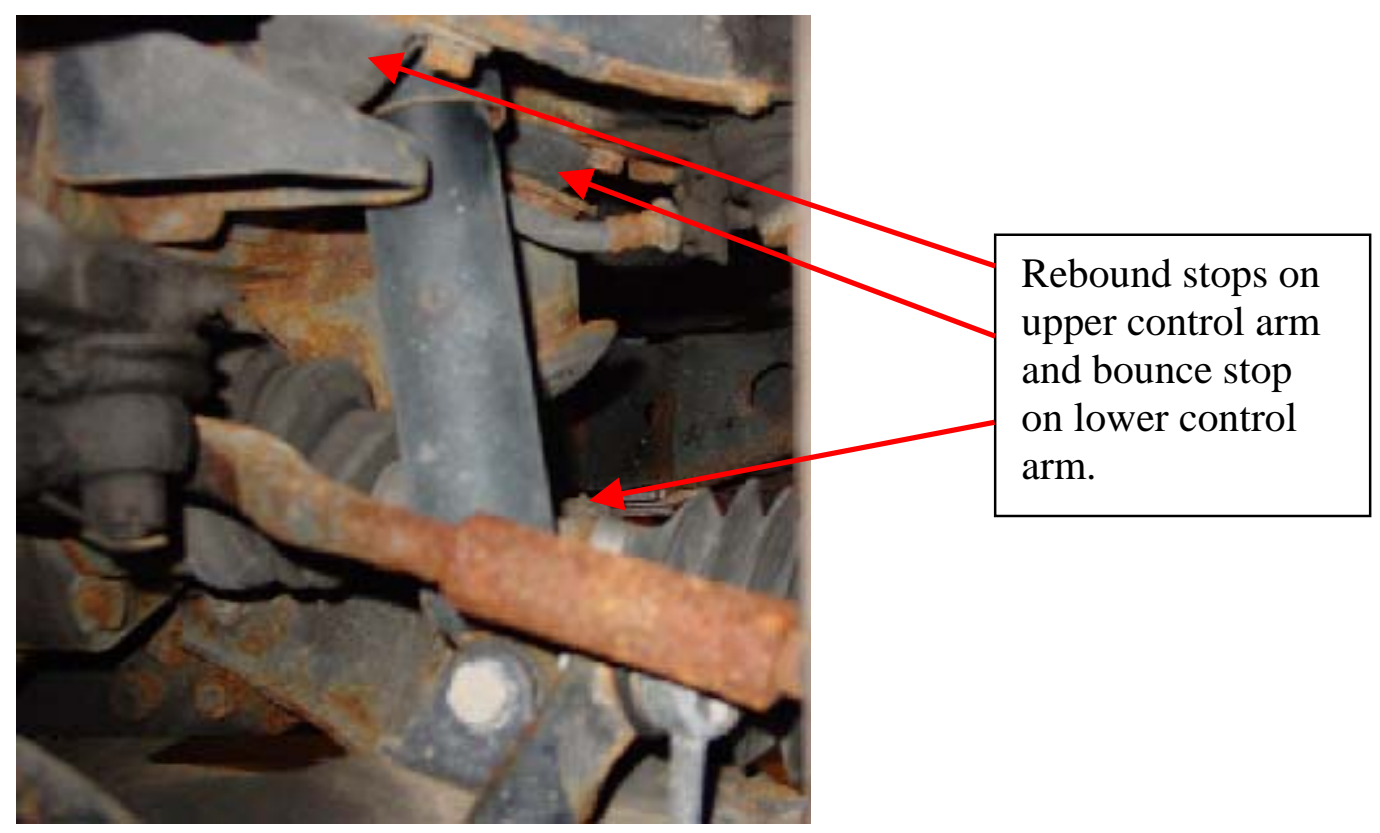

Figure 5.22: Montero Front-Left Suspension

(view facing rearward, wheel in rebound) 


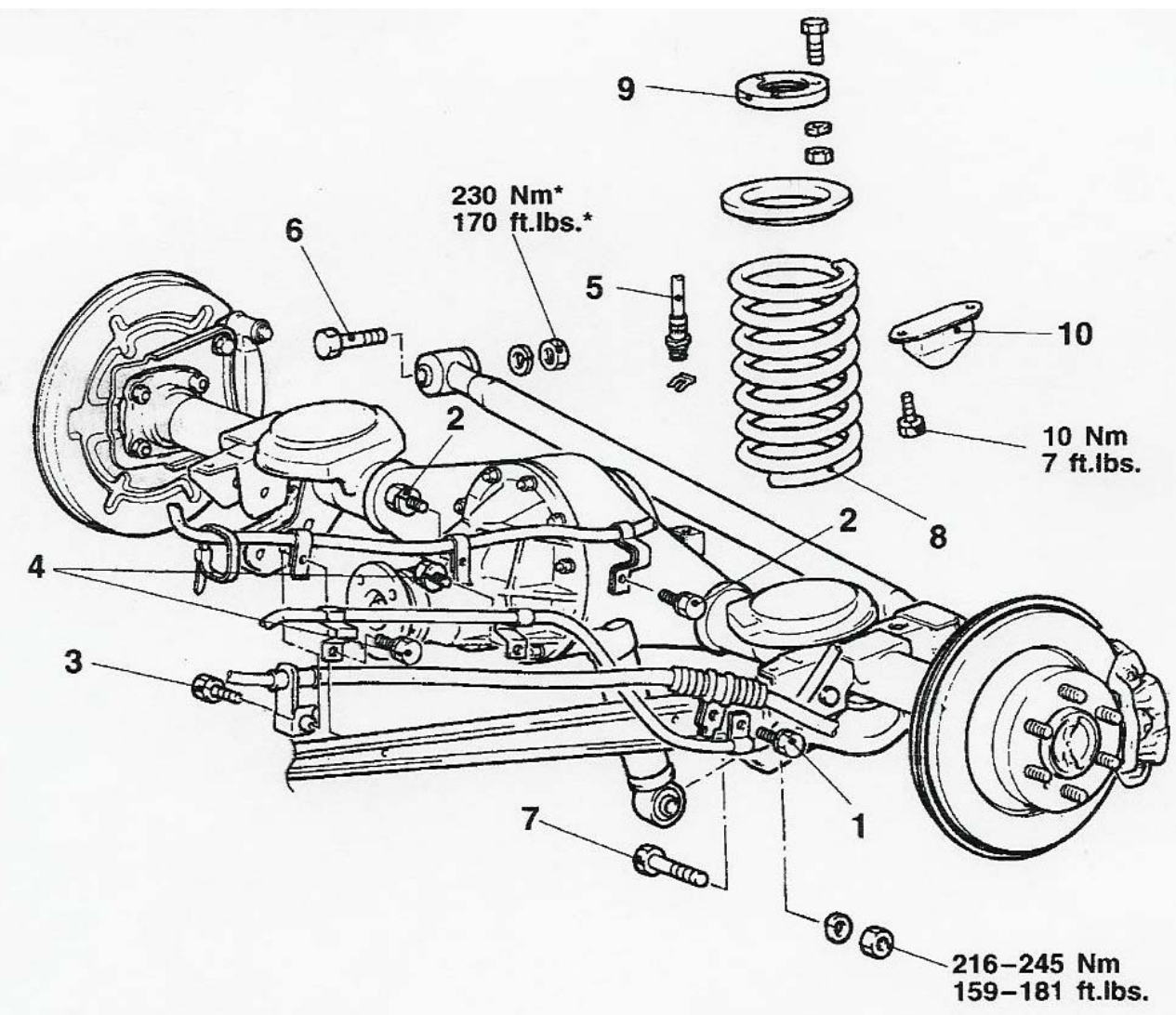

1. Parking brake cable attaching bolt

2. Rear differential lock position harness attaching bolt

3. parking brake cable attaching bolt

4. rear sensor attaching bolt (Vehicles with A.B.S.)

5. Brake hose connection

6 . Lateral rod mounting bolt (body side only)

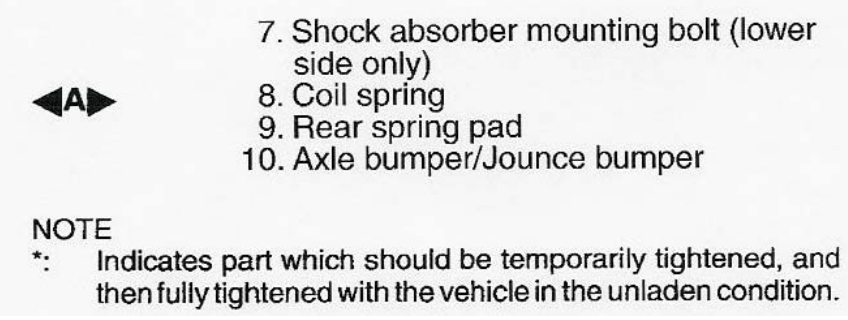

Figure 5.23: Montero Rear Suspension 


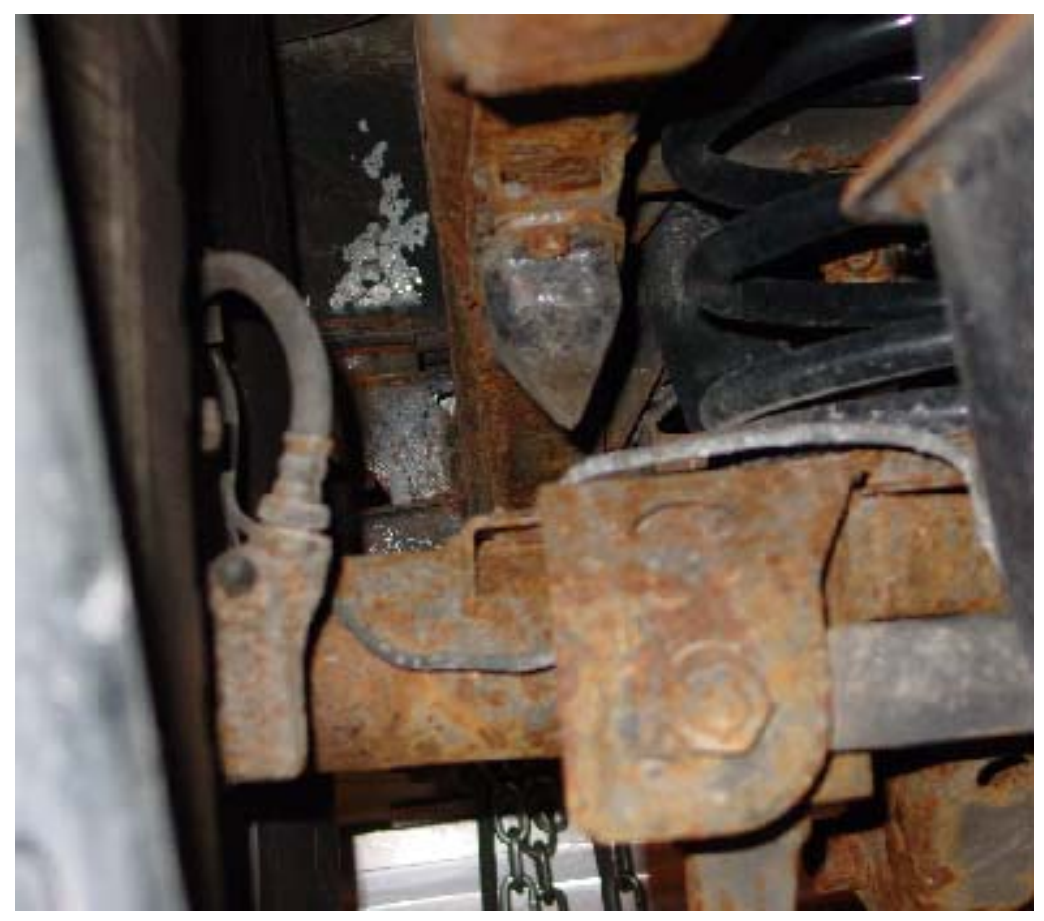

Figure 5.24: Montero Rear-Left Suspension

(view looking forward: bump stop, lateral link (connected to axle), and coil spring) 


\subsection{Physical Suspension Asymmetries}

As shown in Figures 5.11 and 5.21, the front suspensions of both the SLX and the Montero were typical torsion bar suspensions with a stabilizer bar. The general layouts of those suspensions were similar and the only significant difference was that the Montero had rebound stops. As for the rear suspensions, shown in Figures 5.12 and 5.23, both vehicles had a solid axle with coil springs, stabilizer bar, lateral rod, and trailing links. Differences between the two rear suspensions include the shape of the bump stops, presence of a center link (SLX only), and the attachment points of the lateral (Panhard) rod. For the SLX, the left side of the rod was attached to the sprung mass (body) and the right side was attached to unsprung mass (solid axle). The attachment points for the Montero were just the opposite. The left side was attached to the axle, and the right side was attached to the body.

The purpose of the Panhard rod is to distribute the lateral force between the sprung and unsprung masses. When the rod is horizontal, the distributed forces are purely lateral, but upon some inclination of the rod, vertical forces are produced. The magnitude and direction of these vertical forces are dependent on the slope of the rod and the direction of vehicle cornering. As such, the vertical forces can either lift or pull down the sprung mass. Furthermore, [22] states that "some considerations in the design of a track bar [Panhard bar] include whether it is normally in tension or compression. For circle track racing, always turning left means that the bar should be attached to the body on the right side and to the axle on the left assuring that it is always in tension when cornering.” This statement supports the rollover tendencies of the SLX and the Montero. For example, the undesired effect of having the bar in compression occurs when the SLX 
is steering left and when the Montero is steering right, in which both cases were more prone to vehicle rollover relative to the opposite steering direction. In addition, during severe maneuvers, the roll angles can easily be above $8^{\circ}$ (without even producing TWL at $8^{\circ}$ ), and therefore, the inclination of the rod relative to the axle will produce more of a force coupling effect. 


\section{CHAPTER 6}

\section{VEHICLE DYNAMICS SIMULATION USING CARSIM ${ }^{\mathrm{TM}}$}

\subsection{Introduction}

This chapter accomplishes the fifth objective of this research, which is to evaluate the sensitivity of vehicle response to asymmetric suspension characteristics. The vehicle dynamics simulation, CarSim ${ }^{\mathrm{TM}}$, was used for this purpose. More specifically, CarSim ${ }^{\mathrm{TM}}$ was used to evaluate the effect of the suspension asymmetries, detailed in Chapter 5, in an effort to explain the vehicle response asymmetries described in Chapter 4 . The validity of CarSim ${ }^{\mathrm{TM}}$ and the model created in it, as well as the simulation results, are presented in this chapter.

\subsection{Validity of CarSim ${ }^{\mathrm{TM}}$}

CarSim $^{\mathrm{TM}}$ is a PC-based program for simulating the dynamic response of a vehicle. It allows the user to create detailed vehicle models based on vehicle dimensions, inertias, suspension setup and response, steering response, and tire parameters. Version 5.15 was used in this simulation study and the model created (independent front suspension and solid axle rear suspension) is represented mathematically by 54 ordinary differential equations that describe its kinematic and dynamic behavior. It is composed of 9 bodies and has 14 multibody degrees of freedom. 
It would be ideal to create a CarSim ${ }^{\mathrm{TM}}$ model that completely matched the vehicle parameters of the SLX and/or the Montero, perform J-turn tests, and modify the model accordingly until the simulation matched the field test results. Thus the CarSim ${ }^{\mathrm{TM}}$ model of the SLX and/or the Montero would represent the actual vehicles well, and the models would be considered validated. However, a "perfect" model is beyond the scope of this research effort, and it is not needed for the current study. The goal of this simulation effort is simply to gain a better understanding of the effect of the suspension asymmetries on vehicle response. Therefore a representative model is sufficient assuming that CarSim $^{\mathrm{TM}}$ can accurately evaluate the model to produce meaningful results. The developers of CarSim ${ }^{\mathrm{TM}}$, the Mechanical Simulation Corporation (MSC), claim so. MSC states that "CarSim is built on decades of research in characterizing vehicles and reproducing their behavior with mathematical models," and that "its validity has been well established by many automotive OEMs and Tier 1 suppliers where it is currently being used" [27]. Given that and CarSim's ${ }^{\mathrm{TM}}$ popularity in industry and academic research, this research effort assumes that CarSim ${ }^{\mathrm{TM}}$ is capable of representing the vehicle response of mid-sized SUV's like the SLX and the Montero in the J-turn maneuver to evaluate the effect of suspension asymmetries.

\subsection{The Vehicle Model Created in CarSim ${ }^{\mathrm{TM}}$}

As mentioned, a representative model is sufficient for this study. A representative model is assumed to be a model that focuses on the first order effects of the vehicle (e.g., the vehicle masses, CG locations of those masses, and inertias) as well as contain comparable suspension parameters to the actual vehicle. Given the nature of this study, 
only one model is needed. The model created was based on CarSim's ${ }^{\mathrm{TM}}$ Big SUV model (baseline, front steer only, rear wheel drive, independent front suspension, and solid axle rear suspension). In an effort to represent, not necessarily match, the response of the SLX, the model's masses, inertias, and CG locations were modified to represent those of the SLX in the maximum occupancy configuration. As detailed in Chapter 4, this configuration produced the most asymmetric response by the SLX and the Montero in the J-turn maneuver. Unfortunately, CarSim ${ }^{\mathrm{TM}}$ suspension models have only two generic forms: either independent or solid axle. As such, the effect of the Panhard rod as discussed in Section 5.4 cannot be evaluated using CarSim ${ }^{\mathrm{TM}}$.

The total vehicle mass, CG location, and inertias (roll, pitch, and yaw) for the SLX and the Montero (as determined by S.E.A., Inc.) and the models used in CarSim ${ }^{\mathrm{TM}}$ are given in Table 6.1 (baseline values were first presented in Table 2.1). Instead of using the total vehicle values directly, CarSim $^{\mathrm{TM}}$ requires the sprung mass values. CarSim's ${ }^{\text {TM }}$ default unsprung mass values (both front and rear) and their respective CG heights were used, but the lateral and longitudinal CG locations were assumed to be at the wheel centers, i.e., four separate masses represent the unsprung masses. The location of the sprung mass CG was calculated using a static moment equation based on the total vehicle CG location and the assumed unsprung masses CG locations. The roll, pitch, and yaw sprung mass moments of inertia required by CarSim $^{\mathrm{TM}}$ were calculated based on the parallel axis theorem and the known total vehicle properties, with the assumption that the vehicle inertia can be accurately represented by five separate masses: the sprung mass and the unsprung mass at each wheel center. 


\begin{tabular}{|c|c|c|c|c|c|c|c|c|c|c|c|}
\hline \multirow{3}{*}{ Vehicle } & \multirow{3}{*}{ Configuration } & \multirow{3}{*}{ SSF } & \multirow[b]{2}{*}{ Weight } & \multirow[b]{2}{*}{ Wheelbase } & \multirow[b]{2}{*}{$\begin{array}{l}\text { Mean } \\
\text { Track } \\
\text { Width }\end{array}$} & \multicolumn{3}{|c|}{ C.G. } & \multicolumn{3}{|c|}{ Mass Moments of Inertia } \\
\hline & & & & & & Longitudinal & Height & $\begin{array}{c}\text { Lateral } \\
\text { Offset }\end{array}$ & Pitch & Roll & Yaw \\
\hline & & & (lb) & (in) & (in) & (in) & (in) & (in) & (ft-lb-sec $\left.{ }^{2}\right)$ & (ft-lb-sec $\left.{ }^{2}\right)$ & $\left(\mathrm{ft}-\mathrm{lb}-\sec ^{2}\right)$ \\
\hline \multirow{3}{*}{1995 Mitsubishi Montero } & baseline & 0.95 & 4655 & 107.2 & 56.0 & 57.55 & 29.35 & -1.13 & 2859 & 581 & 2920 \\
\hline & nominal load & 0.98 & 4961 & 107.2 & 56.0 & 57.74 & 28.56 & -0.95 & 3198 & 650 & 3317 \\
\hline & maximum occupancy & 0.95 & 5657 & 107.2 & 56.0 & 63.55 & 29.58 & -0.75 & 3489 & 698 & 3618 \\
\hline \multirow{3}{*}{1996 Acura SLX } & baseline & 1.10 & 4467 & 108.5 & 60.0 & 52.13 & 27.33 & -0.51 & 2758 & 572 & 2874 \\
\hline & nominal load & 1.13 & 4798 & 108.5 & 60.0 & 52.56 & 26.58 & -0.46 & 3090 & 640 & 3246 \\
\hline & maximum occupancy & 1.08 & 5327 & 108.5 & 60.0 & 56.06 & 27.74 & -0.55 & 3214 & 677 & 3397 \\
\hline \multirow{2}{*}{ 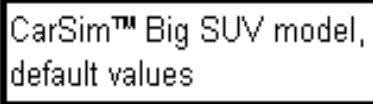 } & total vehicle values & 1.28 & 5401 & 112.2 & 63.8 & 43.50 & 24.88 & 0.00 & 3591 & 1017 & 3662 \\
\hline & sprung mass values & & 4872 & & & 42.13 & 25.98 & 0.00 & 3195 & 917 & 3195 \\
\hline \multirow{2}{*}{$\begin{array}{l}\text { CarSim }^{\top m} \text { SLX model, } \\
\text { maximum occupancy }\end{array}$} & total vehicle values & 1.08 & 5325 & 108.5 & 60.0 & 56.06 & 27.74 & -0.55 & 3217 & 655 & 3531 \\
\hline & sprung mass values & & 4798 & & & 56.26 & 29.18 & -0.61 & 2856 & 553 & 3114 \\
\hline
\end{tabular}

Table 6.1: Vehicle Parameters of Actual Vehicles and CarSim ${ }^{\mathrm{TM}}$ Models 
Upon completion of a simulation run, CarSim ${ }^{\mathrm{TM}}$ outputs a summary (“echo”) file that lists all the variables used, including the total vehicle CG locations and inertias. As shown in Table 6.1, these "total vehicle values" for the SLX model are about the same as the actual SLX values (any differences are highlighted), therefore the equations and assumptions used to calculate the sprung mass properties must be similar to those used in CarSim $^{\mathrm{TM}}$ to determine the total vehicle properties. All of the equations and additional assumptions used to compute these masses, CG locations, and inertias are provided in the Appendix.

A comparable J-turn maneuver was created in CarSim ${ }^{\mathrm{TM}}$ based on the J-turn used in Phase VI for the SLX. For the maximum occupancy load configuration, the SLX's Jturn handwheel magnitude was $384^{\circ}$ and the rate was $1000^{\circ} / \mathrm{sec}$. The model's steering ratio was also modified to that of the SLX to ensure that the model would produce equivalent front wheel steer.

Since CarSim ${ }^{\mathrm{TM}}$ assumes the front suspension parameters are the same for the left and right side, the asymmetric SLX suspension values could not be included in one model/run. Given that, the SLX model used either the front right or front left side suspension parameters to represent the entire front suspension. The corresponding left or right side rear suspension values were used for the entire rear. Thus, the SLX model had either the entire "left side" or "right side" suspension setup, as noted in the figures for the SLX model.

The SLX's suspension spring rates, bump stop stiffnesses, auxiliary roll stiffnesses, and vertical tire stiffnesses were determined from the data presented in Chapter 5 and were included in the model. The actual bounce test data (vertical force 
versus suspension deflection as shown in Figures 5.1 and 5.3) were used as lookup tables in CarSim ${ }^{\mathrm{TM}}$, therefore utilizing the true suspension response rather than using the linearized spring rate and bump stop stiffness values listed in Table 5.2. The front and rear auxiliary roll stiffnesses were extracted from the overall roll stiffnesses according to the procedure used in [28]. The calculated auxiliary stiffness values were used as the "right side" suspension and increased by $10 \%$ for the "left side” values. The front vertical tire stiffnesses were averaged (left and right side) as was the rear tires’ values. Having one vertical tire stiffness value for the front tires and one for the rear tires includes the effect of the different front to rear tire pressures, but ignores the effect of different left to right side values. Based on the bounce test results, the percent differences of the vertical tire stiffness between the front tires is $4.6 \%$ and $1.0 \%$ for the rear tires. For the rear solid axle suspension model, CarSim ${ }^{\mathrm{TM}}$ requires the lateral distance between the springs and the lateral distance between the shocks, of which the SLX values were used. The remaining CarSim ${ }^{\mathrm{TM}}$ suspension and tire parameters were not modified. For reference purposes, the CarSim ${ }^{\mathrm{TM}}$ parsfile for a particular simulation run with the SLX model is included in the Appendix and lists all the default and modified values for that run.

In addition to the vehicle model, the road surface coefficient of friction ( $\mu$ ) was modified so that the SLX model response would be similar to the field tests with respect to the yaw rate (level off around $40 \%$ sec, refer to Figures $4.7-4.10$ ). As shown in Figure 6.1, a $\mu$ value of 1.1 minimized the yaw rate (compared to $\mu=1.0$ ) and did not produce immediate vehicle rollover (compared to $\mu=1.2$ ). As such, $\mu$ was set to 1.1 for all the simulation runs. The CarSim ${ }^{\mathrm{TM}}$ default Big SUV tire model stated "tire/ground friction 
coefficient for this [tire] data" as 0.87 for lateral force data and 0.89 for longitudinal force data. It was assumed that CarSim $^{\mathrm{TM}}$ multiplies $\mu$ with the tire/ground friction coefficient to get an overall friction coefficient; therefore the overall friction coefficient, with $\mu$ of 1.1, is 0.974 for lateral forces and 0.957 for longitudinal forces. This seems reasonable since the VDA's range of peak SN was 0.95 - 0.99 during the SLX and Montero testing.

\subsection{Simulation Results}

Given the vast amount of vehicle response variables produced by CarSim $^{\mathrm{TM}}$ and the focus of this research on rollover maneuvers (TWL), the format of the graphs shown in this chapter have been modified from the Chapter 4 graphs to include vertical wheel displacements for the front and rear tires (in place of yaw acceleration and roll acceleration plots that were shown previously).

The vertical wheel displacement values are given in the "Front (and Rear) Wheel Lift” plots. Each of these dynamic values is the current wheel center height minus the average of that wheel center height for the first second of the test, i.e., wheel lift is defined as the wheel center height minus the rolling radius of the tire. Given the vertical tire deflection, the stated wheel lift values are not necessarily the distance between the tire tread and the road surface. Based on the average tire stiffness (1370.3 lb/in) and vertical load (1331.8 lb), the average vertical tire deflection is $0.97 \mathrm{inch}$. Thus, the tire loses lateral grip as the "wheel lift" approaches one inch and upon reaching one inch, loses complete contact with the ground. For ease of comparison, only those wheels that are likely to tip up (inside wheels) are shown in the wheel lift plots, e.g., the left side wheels are shown for the left steer J-turn and vice versa for the right side. A legend is 
provided in the wheel lift plots as additional clarification when left and right steer maneuvers are plotted together. For ease of comparison, all values are shown as absolute (positive).

As a basis for comparison, the default CarSim ${ }^{\mathrm{TM}}$ Big SUV (baseline, only front steer, rear wheel drive only) model response is shown in Figure 6.2. The modified Big SUV model representing the SLX is referred to as the "SLX model."

Based on the vehicle response figures and the CarSim ${ }^{\mathrm{TM}}$ animation, the abrupt and momentary drop in lateral acceleration between 1.2 and 1.4 seconds is caused by the onset of the inside rear wheel lift. This phenomenon occurred in every J-turn simulation run. 

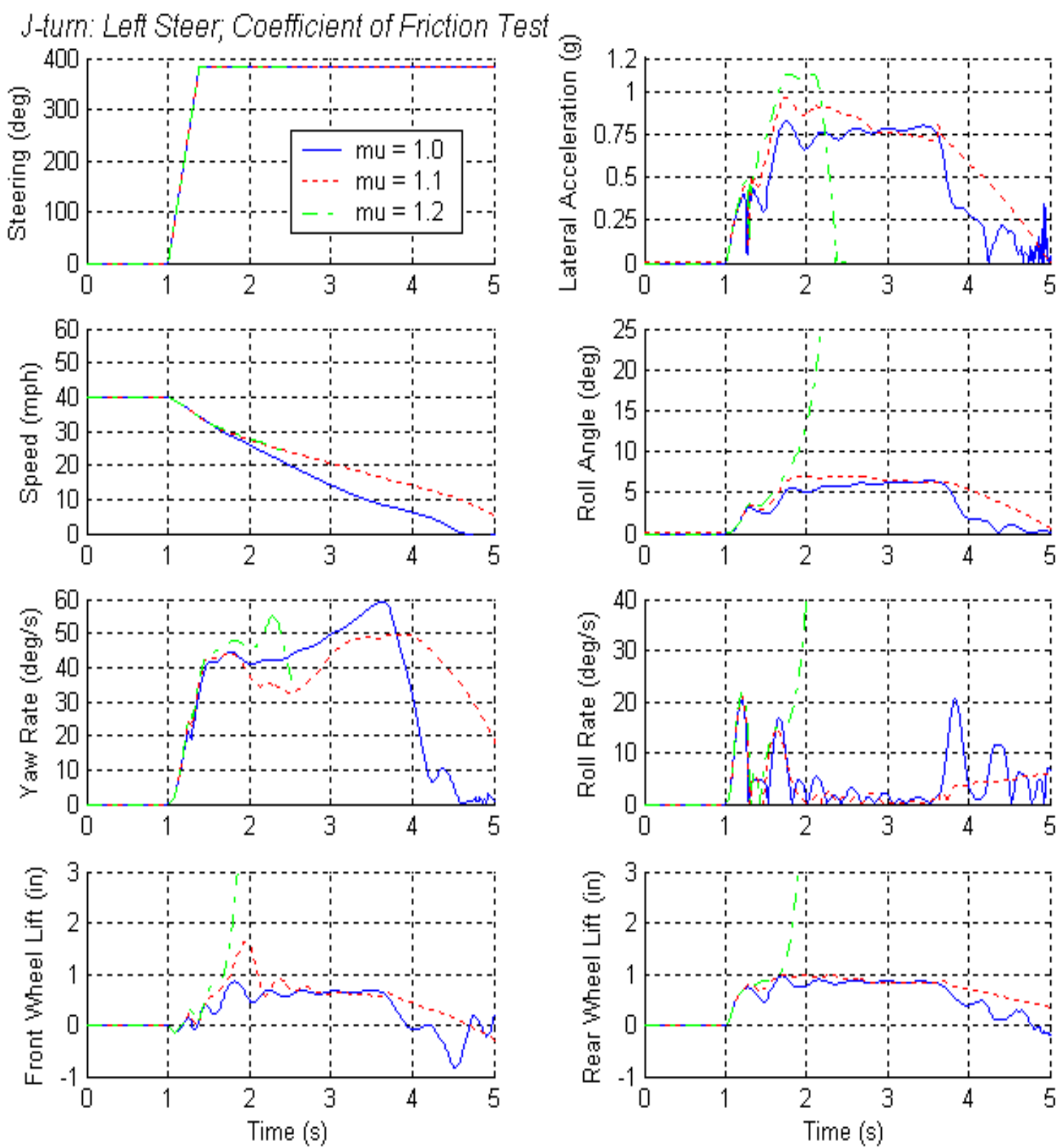

Figure 6.1: Coefficient of Friction Study, SLX Model at $40 \mathrm{mph}$ "Right Side" Suspension, No Lateral CG Offset 

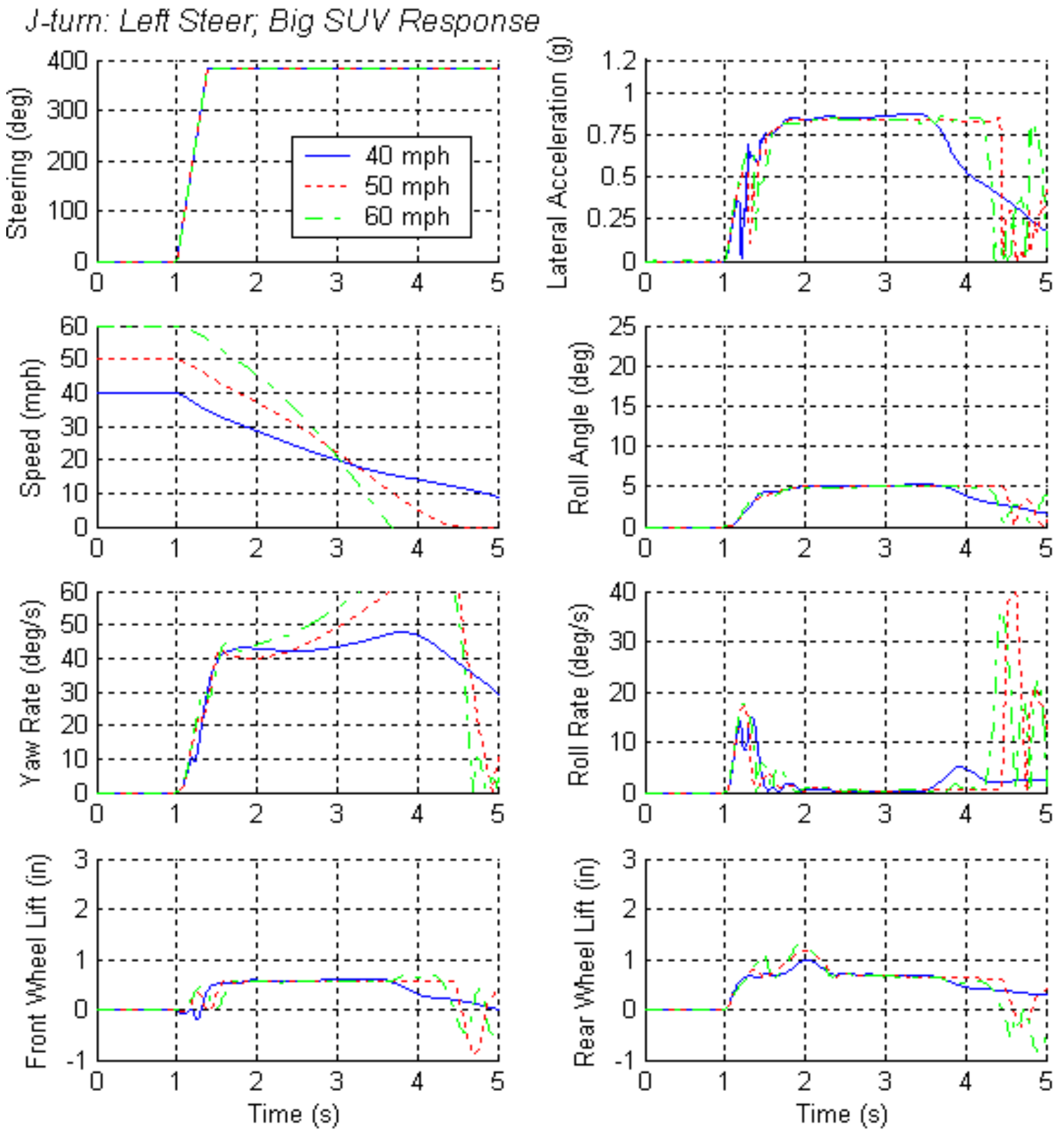

Figure 6.2: Big SUV Baseline Model at 40, 50, and $60 \mathrm{mph}$ 
The lateral CG offset for the SLX model caused the vehicle to drift to the left (toward the CG offset). At $30 \mathrm{mph}$ (top plot in Figure 6.3), the vehicle drifted laterally $0.43 \mathrm{ft}$ (5.2 in) after $40 \mathrm{ft}$ of longitudinal travel. During this time, the initial response values were: roll angle (- $0.16^{\circ}$, independent of speed and handwheel steer correction), yaw rate $\left(-0.08^{\circ} / \mathrm{sec}\right)$, and lateral acceleration $(-0.002 \mathrm{~g})$. Although, the lateral drift is barely noticeable at $30 \mathrm{mph}$, it becomes more apparent at $60 \mathrm{mph}$ (essentially doubles). A handwheel steer correction of $0.4^{\circ}$ (to the right) was used to maintain the vehicle in a straight line before the initial maneuver steer (refer to the bottom plot in Figure 6.3). With the handwheel steer correction, the initial yaw rate $\left(4.6 \mathrm{E}-4^{\circ} / \mathrm{sec}\right)$ and lateral acceleration (1.0E-5 g) values were negligible. All runs that include the lateral CG offset also have the handwheel steer correction (except for the top plot in Figure 6.3)

To test the effect of the lateral CG offset, only the "right side" suspension was used. As shown in Figures 6.4 and 6.5, there was a slight difference in response at 30 mph and a significant difference at $40 \mathrm{mph}$. At that higher speed, both steer directions produced $1.0 \mathrm{~g}$ of lateral acceleration, but the right steer also caused vehicle rollover while the left steer only produced $7^{\circ}$ of roll.

Given the significant effect of the lateral CG offset, the offset was not used in the evaluation of the effect of the asymmetric suspension parameters. Based on the softer versus stiffer side of the suspension discussion in Chapter 5, the "right side" suspension was used for the left steer maneuver and the "left side" suspension for the right steer. At $30 \mathrm{mph}$ (Figure 6.6), there was no noticeable difference between the left and right steer maneuvers. At $60 \mathrm{mph}$, the right steer maneuver with the stiffer suspension produced 
$0.5^{\circ}$ less roll and about $0.1-0.2$ inches less of wheel lift. This slight asymmetric response of the model is negligible when compared to the differences shown in the field test data.

The combined effect of the lateral CG offset and the asymmetric suspension parameters at 30 and $40 \mathrm{mph}$ is shown in Figures 6.8 and 6.9, respectively. There is negligible difference at $30 \mathrm{mph}$, but at $40 \mathrm{mph}$ the right steer maneuver produces vehicle rollover. When compared to the right steer maneuver at $40 \mathrm{mph}$ with the lateral CG offset and the "right side" suspension (Figure 6.5), the stiffer left suspension (Figure 6.9) delays rollover by 0.5 seconds, but all of the response magnitudes are the same.

Based on this simulation study, the lateral CG offset dominated the vehicle response. Using the actual suspension parameters (spring force versus deflection data, and auxiliary roll stiffness) for the SLX showed some differences between left and right steer response, but to a much lesser extent than the CG lateral offset. The model response in roll either leveled off around $5-7^{\circ}$ or completely rolled over. The actual SLX suspension was fairly symmetric in the linear range, but showed differences upon extreme suspension compression (where bump stop contact occurs). Given that, the bump stops may not have had an effect on the model. In the field tests, the SLX frequently experienced roll angles of $10^{\circ}$ without TWL and had an oscillatory roll response that is indicative of bump stop contact. It is unfortunate that the model seems to be missing that middle ground, i.e, early level off of response or complete rollover.

Unfortunately due to time and monetary constraints, the response of the shock absorbers was not determined during the suspension and steering tests described in Chapter 5. To study the effect of the shock absorbers on vehicle response, the default 
damping force, as shown in Figure 6.10, was decreased by 10\% (Figure 6.12) and increased by 10\% (Figure 6.13) and 20\% (Figure 6.14) for the "left side" suspension. In addition, to test the effect of different front to rear shock absorbers, a series of simulations were performed with only the front (Figure 6.15) or rear (Figure 6.16) damping force increased by $10 \%$. All of the shock absorber study tests included the lateral CG offset and the "left side” suspension for a series of right steer J-turn maneuvers at 40, 50, and $60 \mathrm{mph}$. This combination was chosen because it is prone to rollover. For comparison purposes, Figure 6.11 shows the vehicle response with the default damping values. In addition to the vehicle response plots shown previously, the figures in this study include a plot of the vehicle's trajectory.

Compared to the vehicle response with the default damping force, decreasing the damping force by $10 \%$ caused the vehicle to roll over about half a second sooner, but increasing the damping force prevented rollover. As shown in Figure 6.13, at $50 \mathrm{mph}$ the model with the $10 \%$ increase in damping force achieved TWL and $9^{\circ}$ of roll without rolling over, which is a significant difference compared to all of the previous results. Furthermore, the vehicle did not experience TWL when the entrance speed was increased to $60 \mathrm{mph}$. In Phase VI testing, it was assumed that once TWL occurred, any increase in maneuver entrance speed would also produce TWL. All of the simulation runs support this assumption, except for the current one under consideration (Figure 6.13). In this particular simulation run, the increased speed caused the vehicle to plow out, and thus the additional speed was quickly scrubbed off. It is interesting to note that aside from the yaw rate, the vehicle response at 40 and $60 \mathrm{mph}$ is very similar in Figure 6.13. When 
compared to the $10 \%$ increase, the $20 \%$ increase in damping force prevented TWL and reduced the roll angle achieved in the $50 \mathrm{mph}$ simulation, but had little affect on the other plotted values.

Increasing only the rear default damping force prevented the vehicle from rolling over at $40 \mathrm{mph}$, but the response was similar at both 50 and $60 \mathrm{mph}$ to the default damping values. Compared to increasing both the front and rear by $10 \%$, the rear increase only simulations had similar results at $40 \mathrm{mph}$. When compared to the rear increase only, the front increase only simulations produced more wheel lift at $40 \mathrm{mph}$ and delayed rollover by almost half a second in the 50 and 60 mph runs.

Note that given the oscillatory roll response of the SLX observed during the J-turn and road edge recovery maneuvers in Phase VI, it is possible the default damping forces may not have paired well with the spring stiffnesses. If the springs were excessively stiff relative to the shock absorbers, the shocks' damping forces may have been "overpowered" by their respective springs, thereby having a relatively small effect on the overall vehicle response.

Based on the shock absorber study, it is evident that a change in the damping force response can have a significant effect on the vehicle response. A summary of this effect is given in Table 6.2. 


\begin{tabular}{|l|l|}
\hline Change* & Effect \\
\hline$-10 \%$ & vehicle rollover at $2.7-3.0$ seconds \\
\hline default & vehicle rollover at $3.0-3.5$ seconds \\
\hline$+10 \%$ & no vehicle rollover, but TWL at $50 \mathrm{mph}$ \\
\hline$+20 \%$ & no vehicle rollover, and only slight wheel lift \\
\hline$+10 \%$ front & prevent rollover at $40 \mathrm{mph}$, rollover at 50 and $60 \mathrm{mph}$ at $3.3-3.5$ seconds \\
\hline$+10 \%$ rear & prevent rollover at $40 \mathrm{mph}$, rollover at 50 and $60 \mathrm{mph}$ at $2.9-3.0$ seconds \\
\hline
\end{tabular}

Table 6.2: Shock Absorber Study 

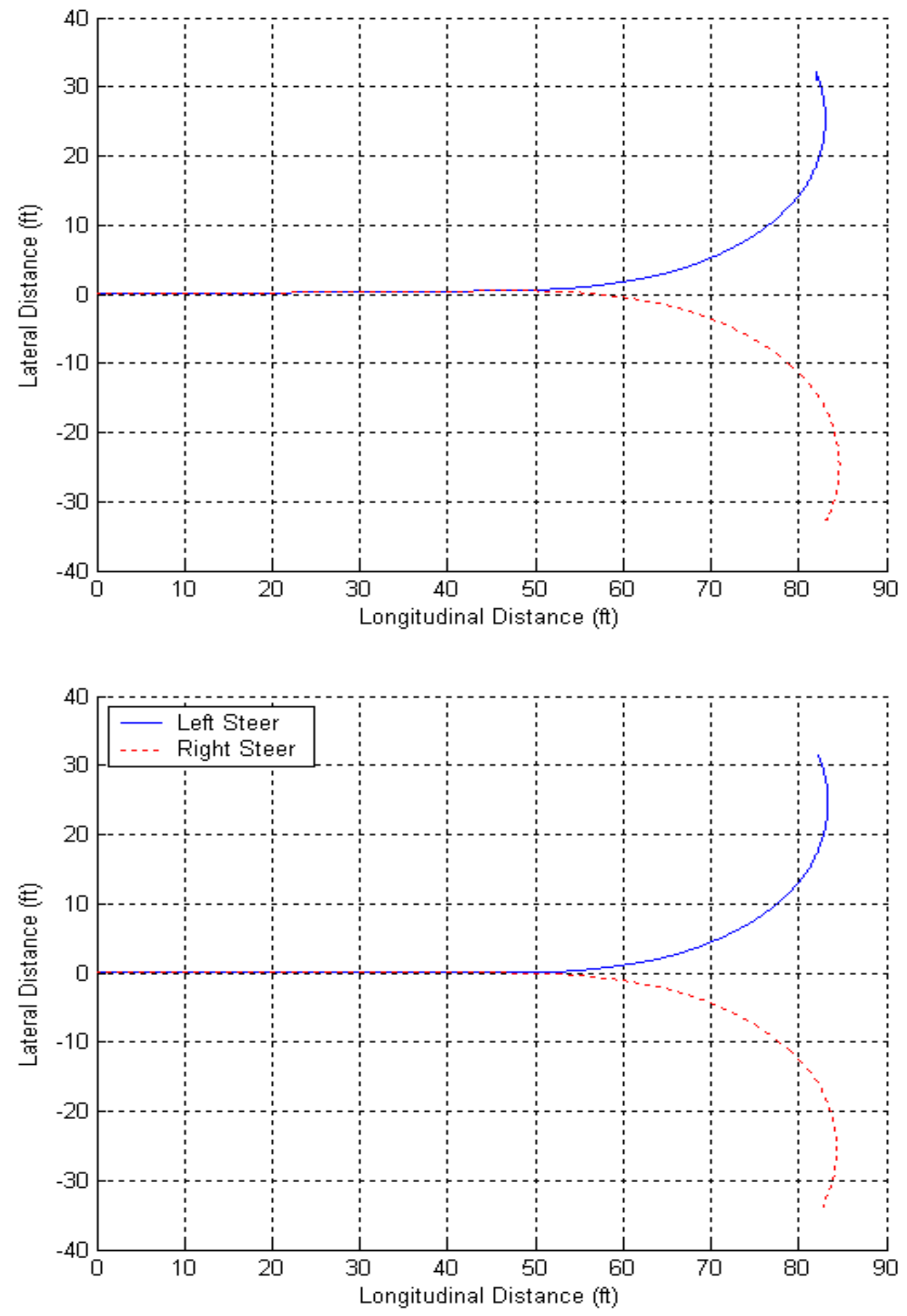

Figure 6.3: SLX Model: Y versus X Trajectory, Effect of the Lateral CG Offset Without (top) and With (top) Handwheel Angle Correction, $30 \mathrm{mph}$ 

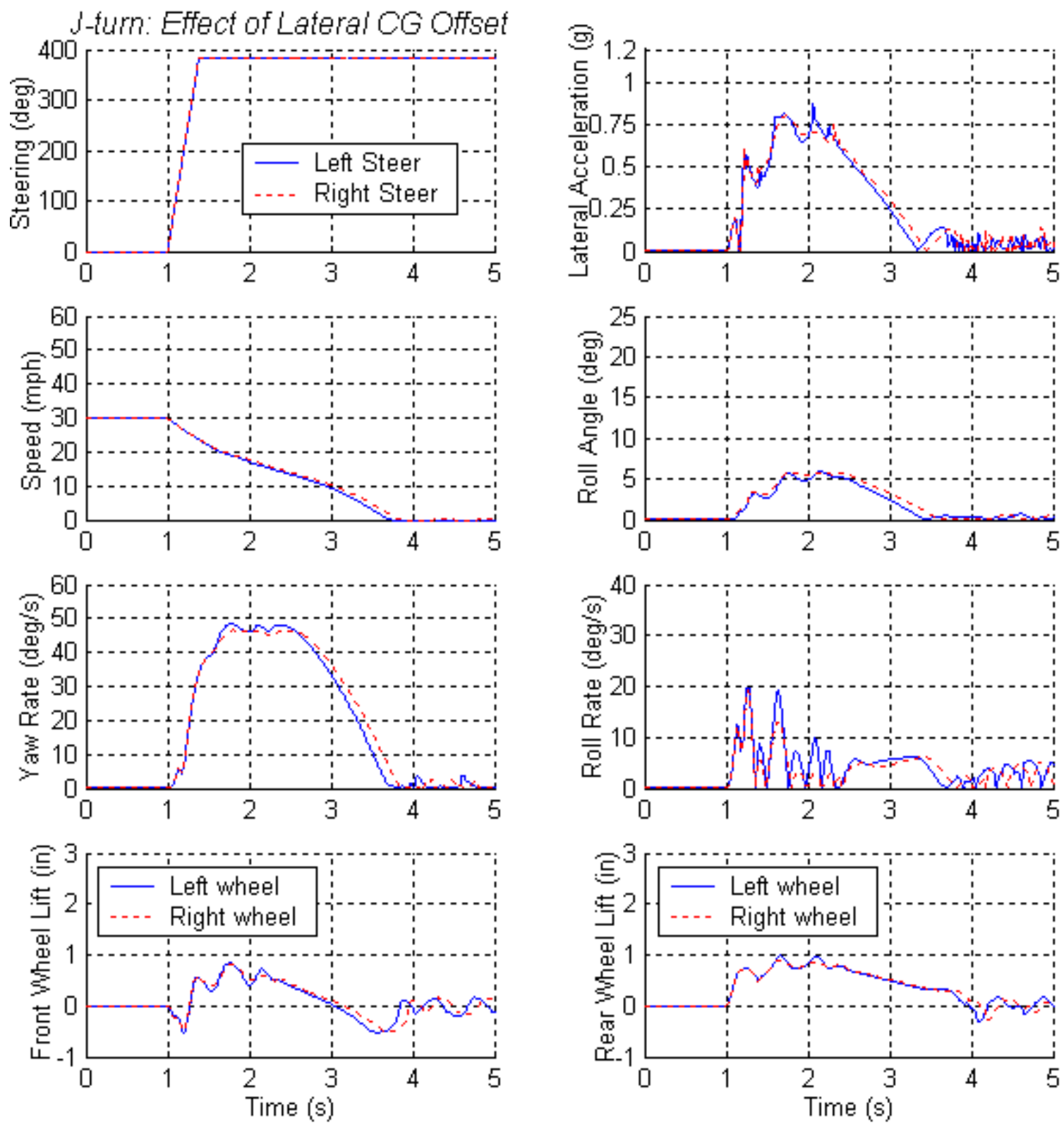

Figure 6.4: SLX Model: Effect of Lateral CG Offset at $30 \mathrm{mph}$ “Right Side” Suspension 

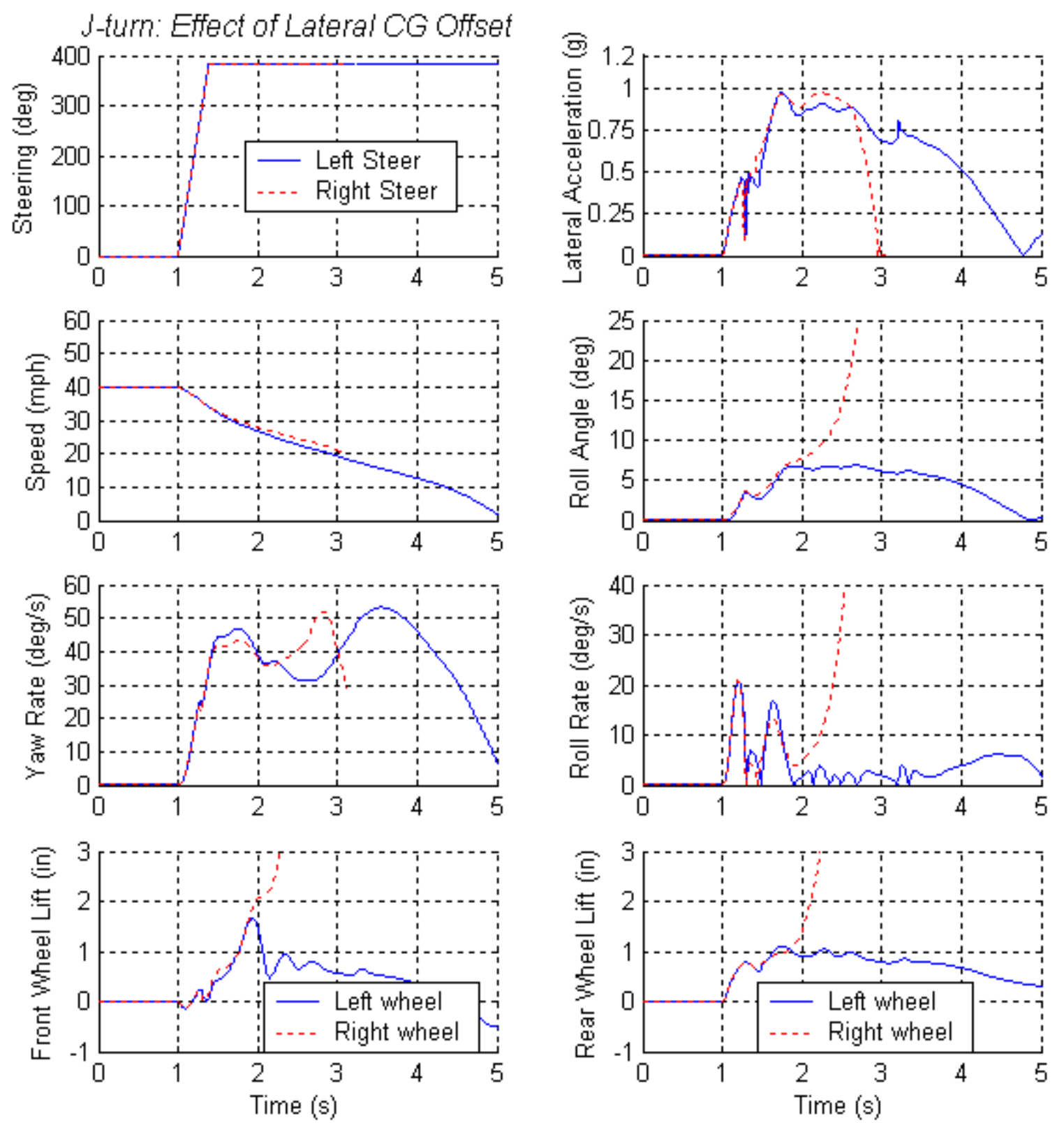

Figure 6.5: SLX Model: Effect of Lateral CG Offset at $40 \mathrm{mph}$ “Right Side” Suspension 

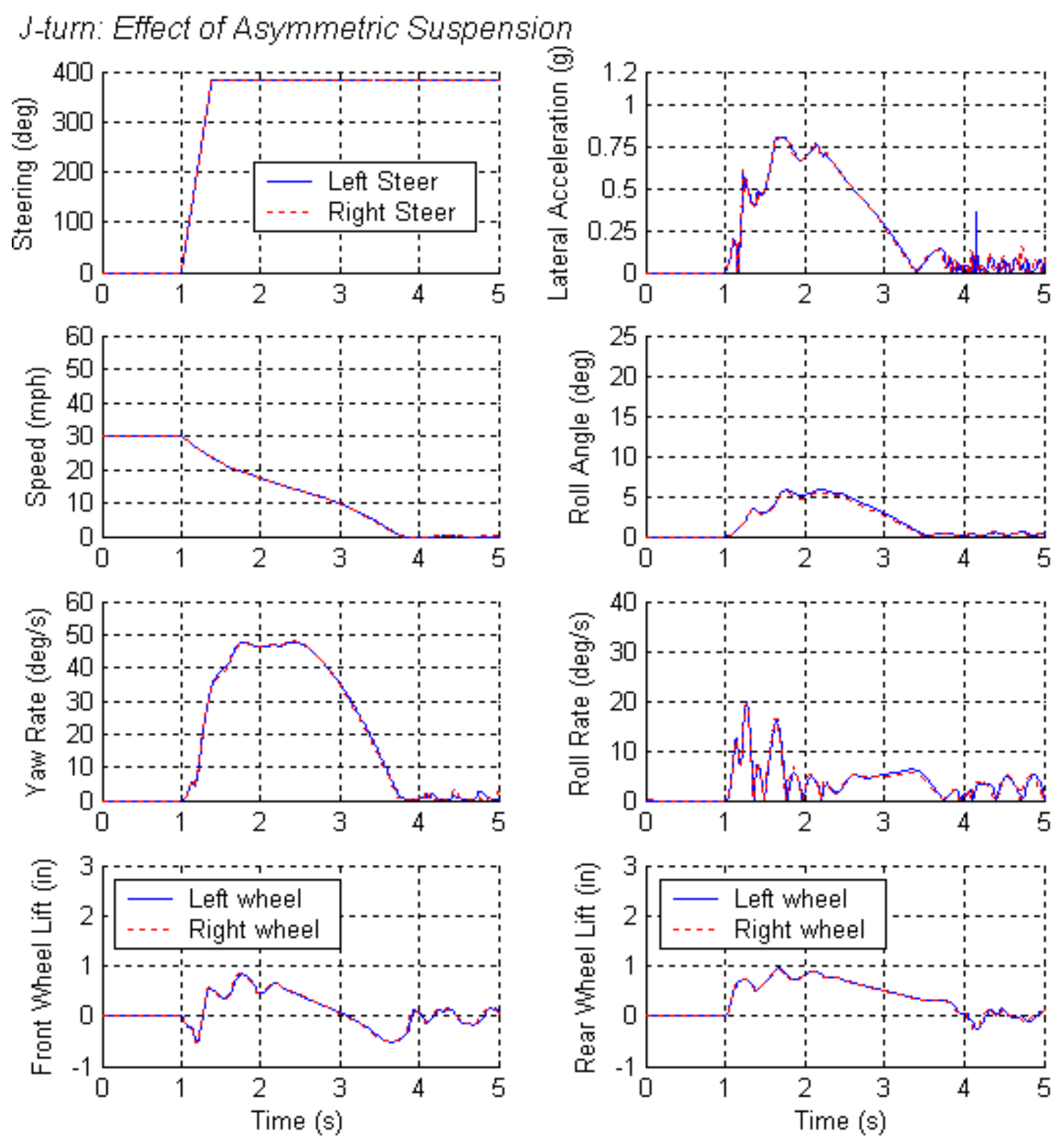

Figure 6.6: SLX Model: Effect of Asymmetric Suspension at $30 \mathrm{mph}$ No Lateral CG Offset

"Right Side” Suspension for Left Steer and "Left Side” Suspension for Right Steer 

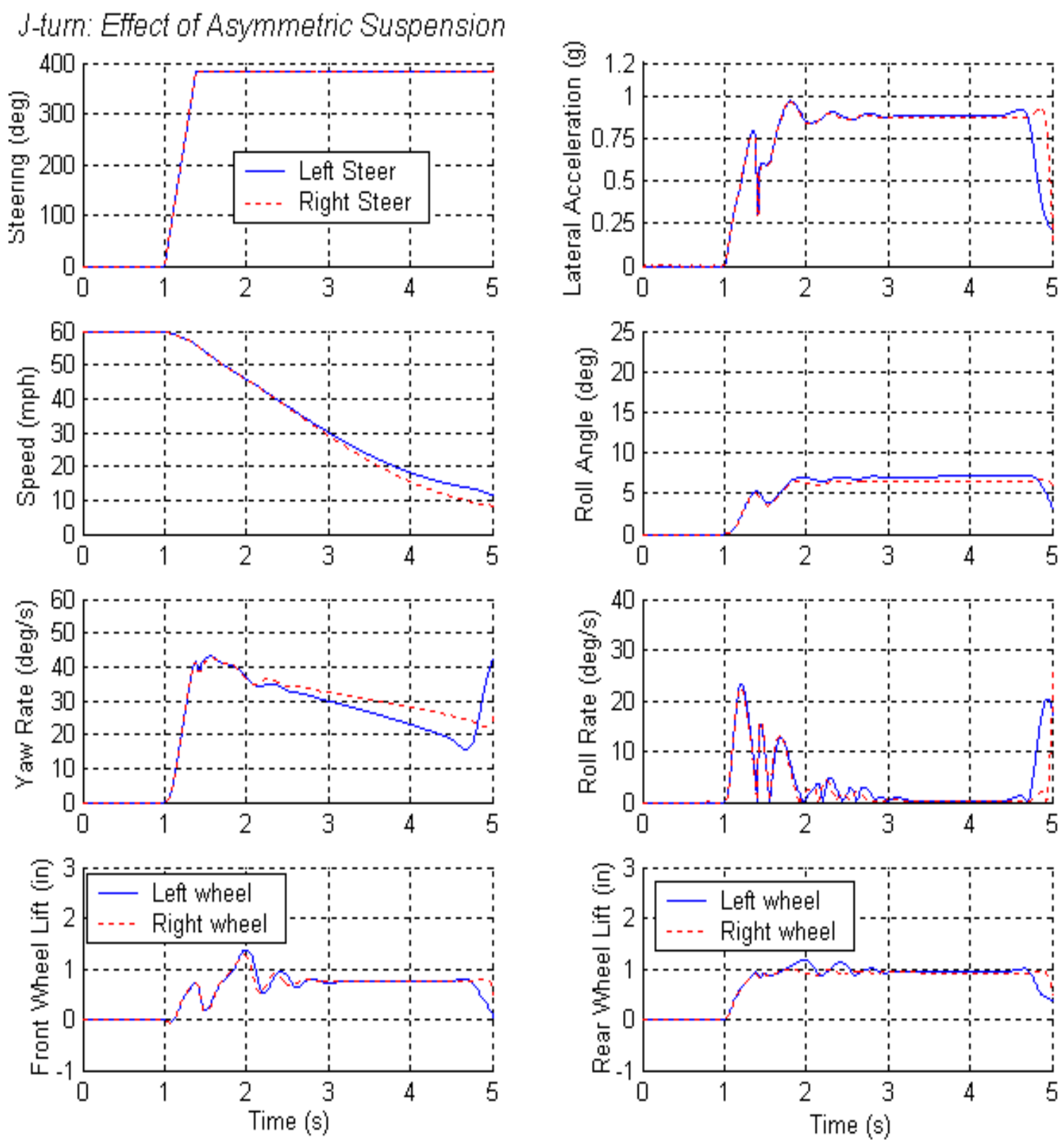

Figure 6.7: SLX Model: Effect of Asymmetric Suspension at $60 \mathrm{mph}$ No Lateral CG Offset

"Right Side” Suspension for Left Steer and "Left Side” Suspension for Right Steer 

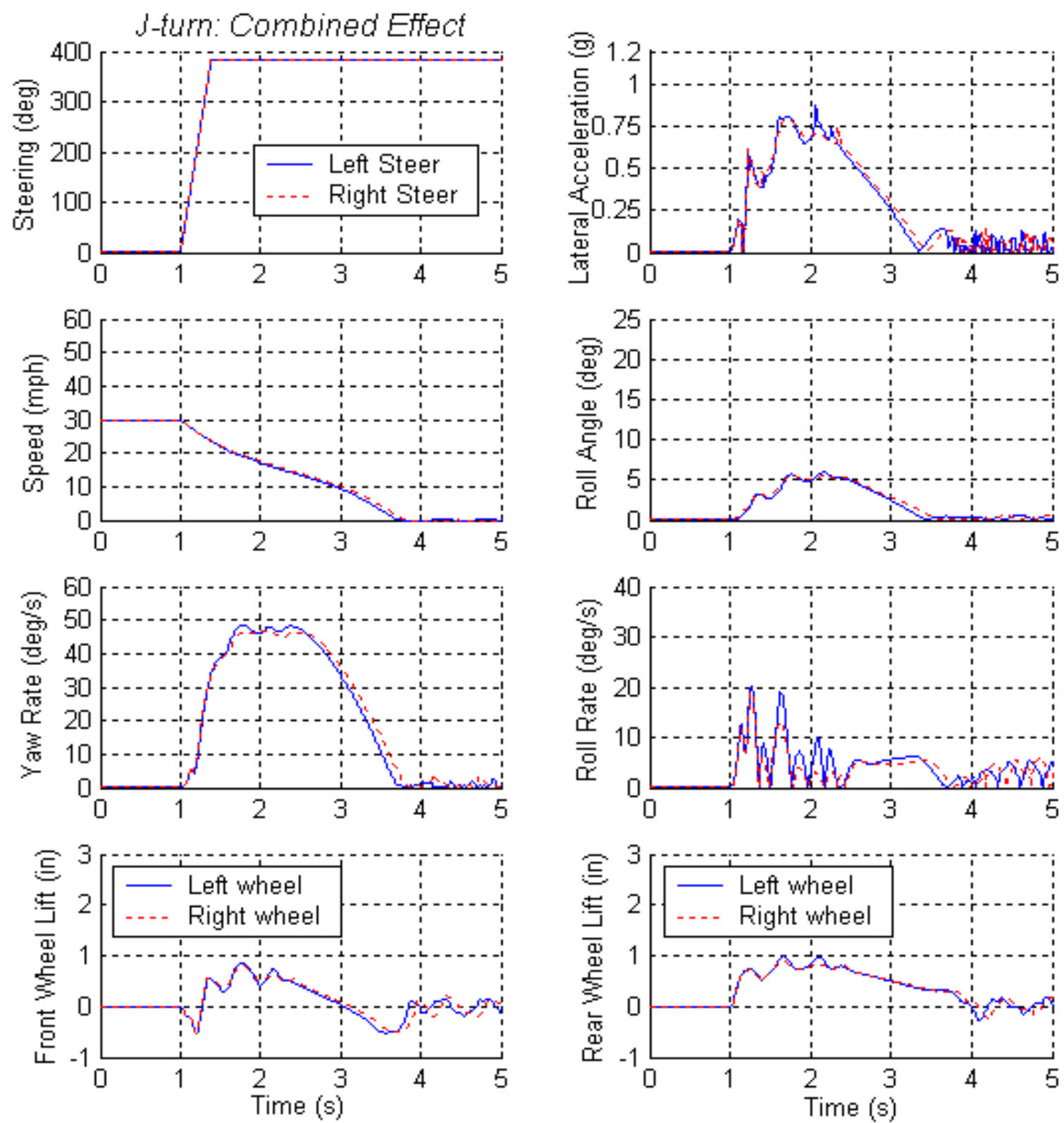

Figure 6.8: SLX Model: Effect of Asymmetric Suspension Parameters and Lateral CG Offset at $30 \mathrm{mph}$ "Right Side” Suspension for Left Steer, and "Left Side” Suspension for Right Steer 

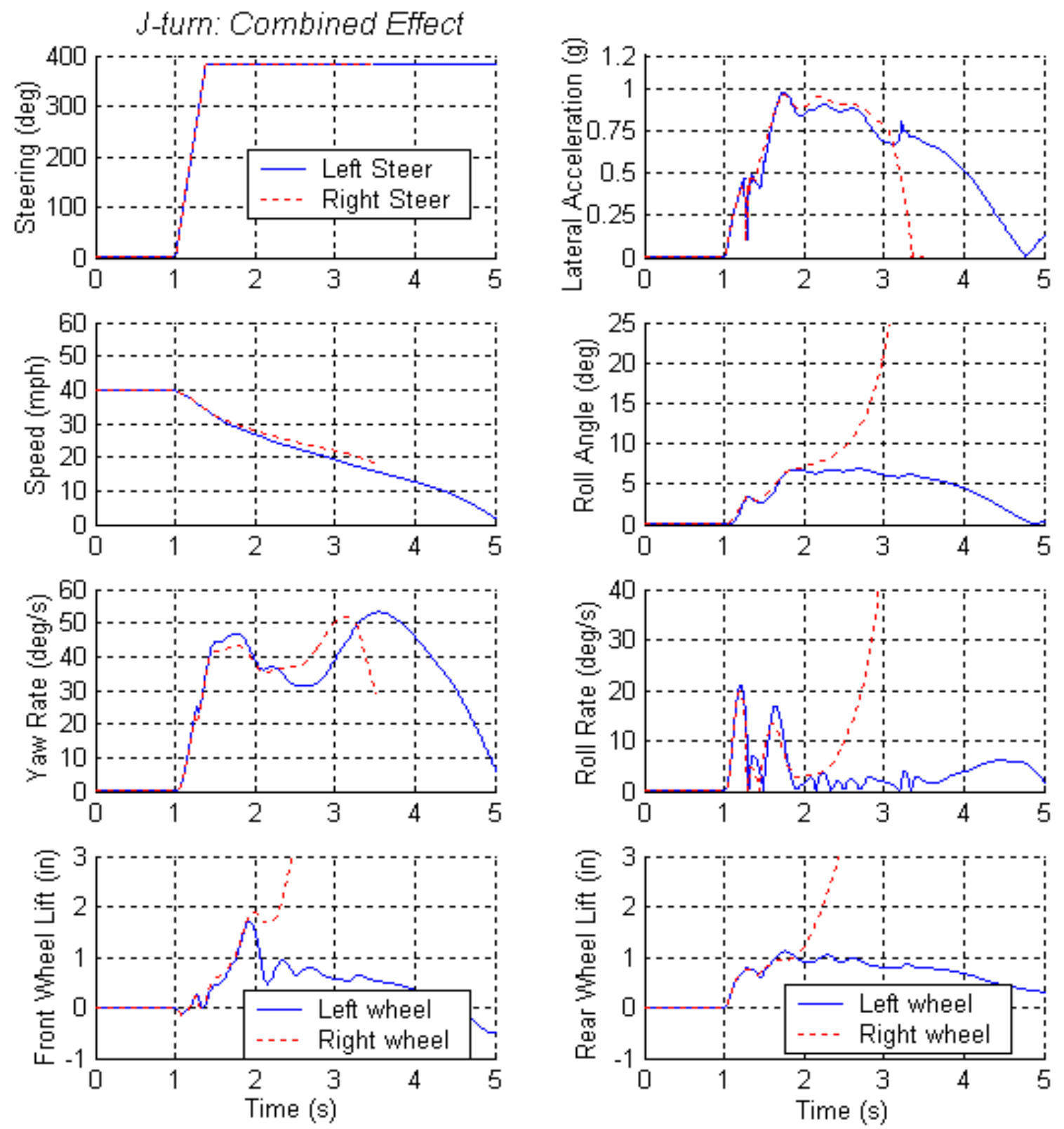

Figure 6.9: SLX Model: Effect of Asymmetric Suspension Parameters and Lateral CG Offset at $40 \mathrm{mph}$

"Right Side” Suspension for Left Steer, and “Left Side” Suspension for Right Steer 


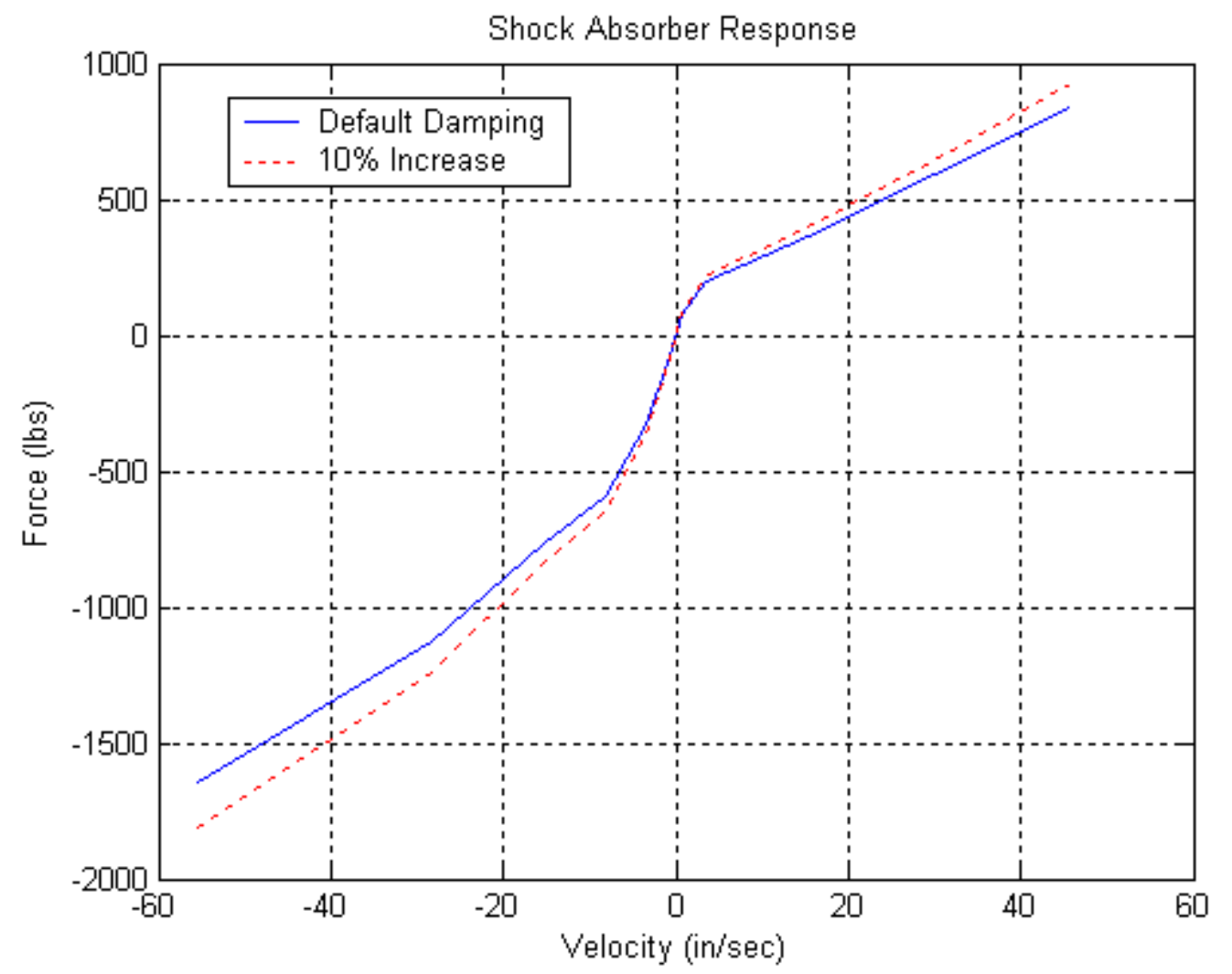

Figure 6.10: CarSim ${ }^{\mathrm{TM}}$ Default Shock Absorber Response 

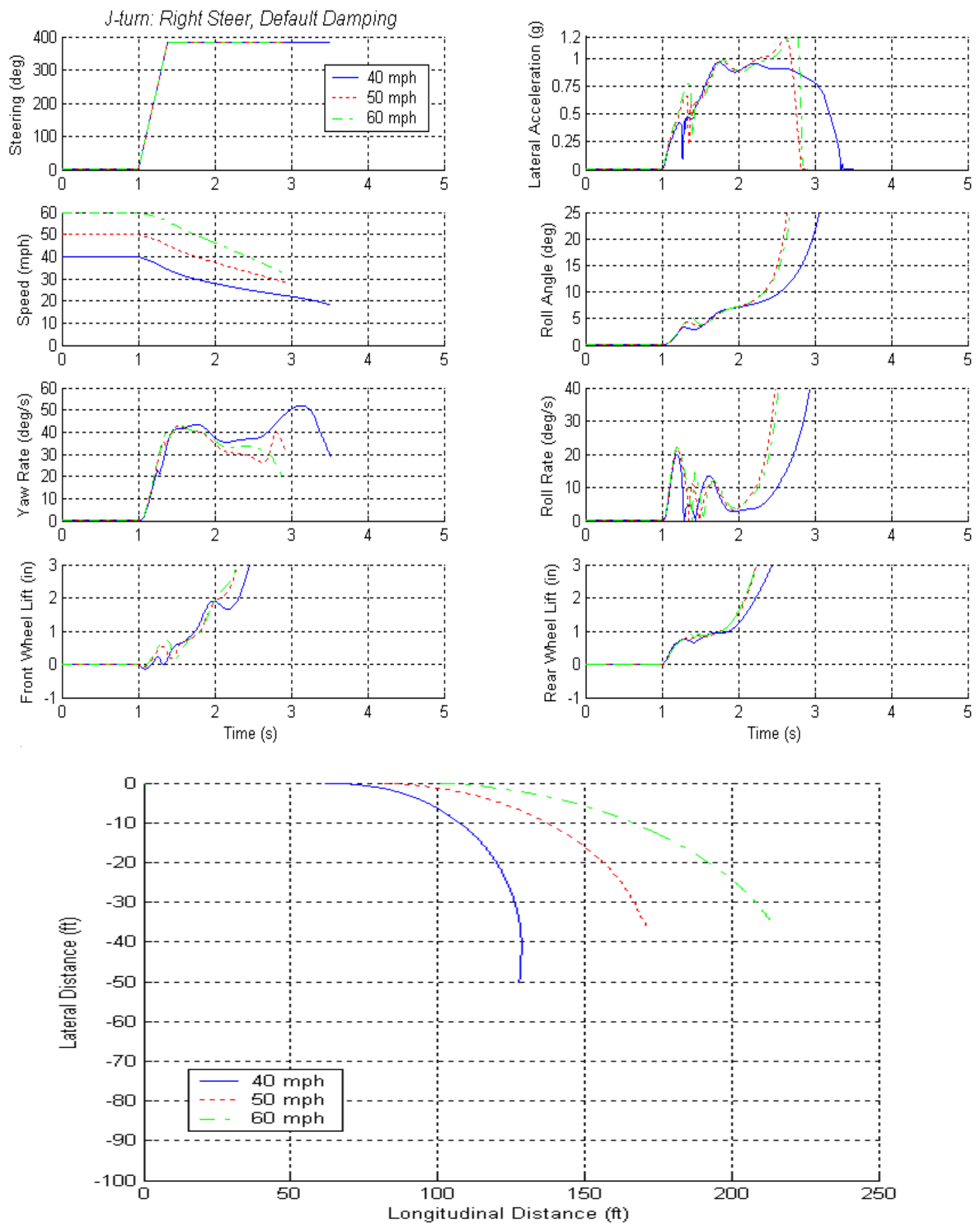

Figure 6.11: Shock Absorber Study, SLX Model "Left Side" Suspension with Lateral CG Offset Default Damping Force for Comparison 

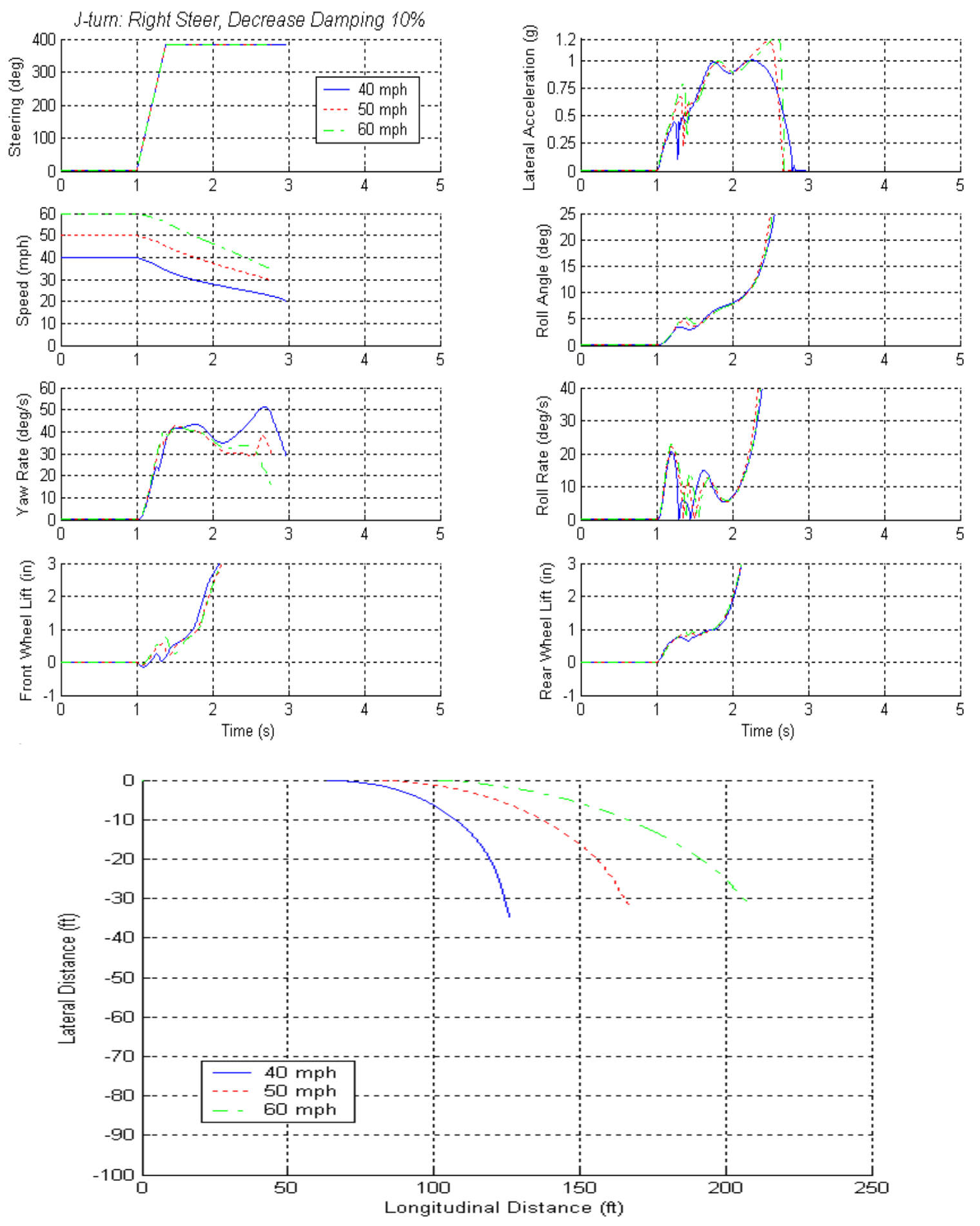

Figure 6.12: Shock Absorber Study, SLX Model "Left Side" Suspension with Lateral CG Offset Decrease the Default Damping Force by 10\% 

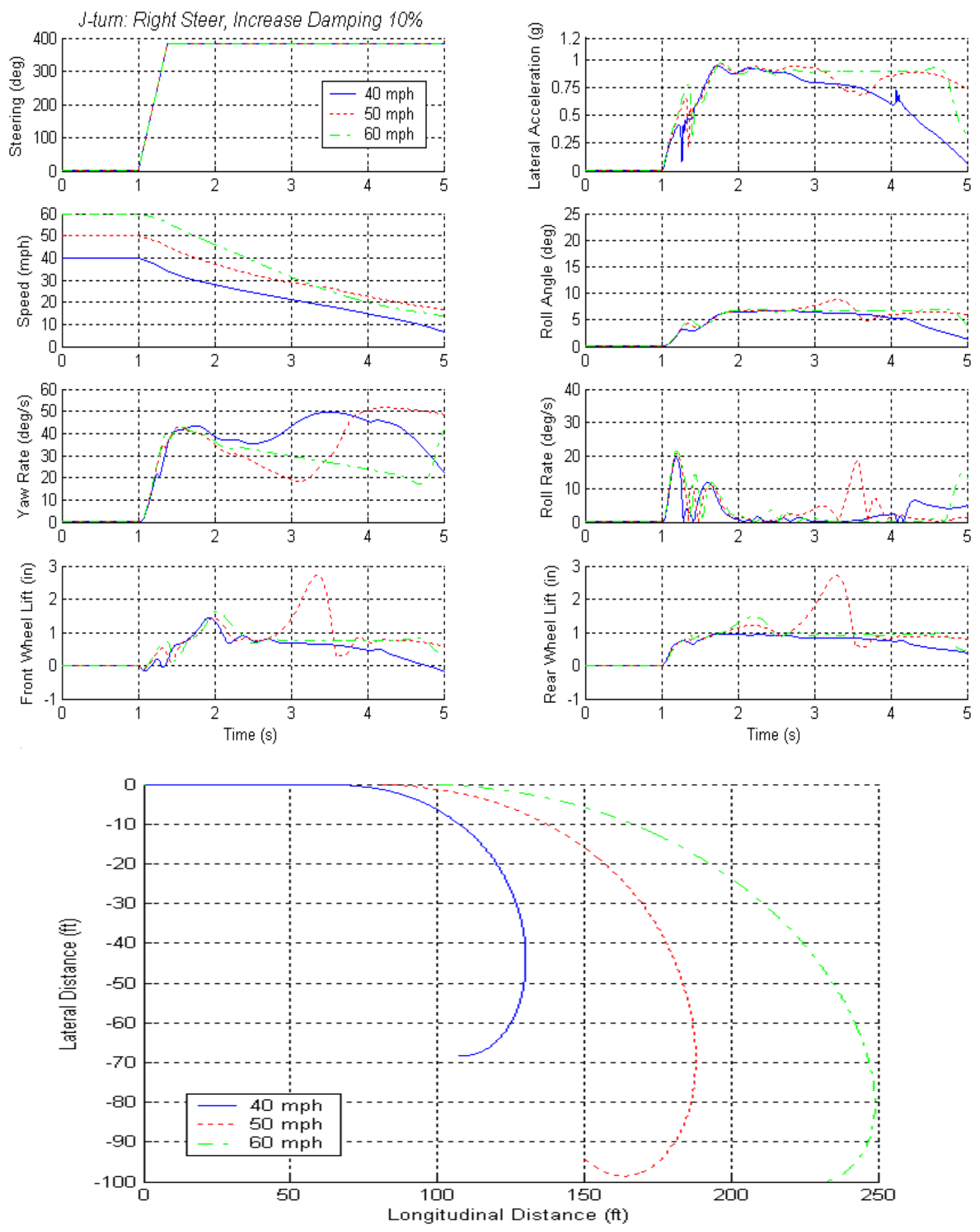

Figure 6.13: Shock Absorber Study, SLX Model "Left Side" Suspension with Lateral CG Offset Increase the Default Damping Force by 10\% 

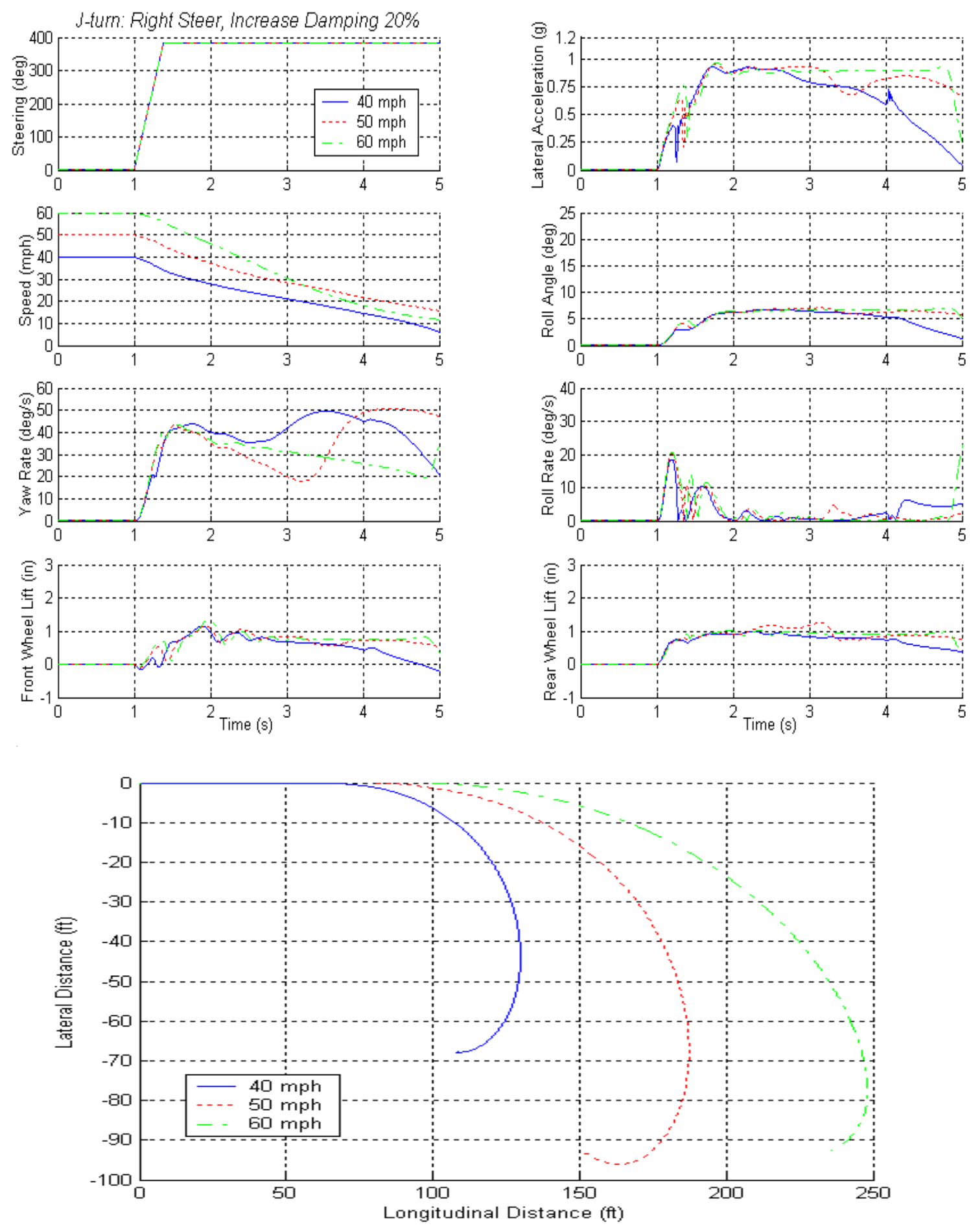

Figure 6.14: Shock Absorber Study, SLX Model "Left Side" Suspension with Lateral CG Offset Increase the Default Damping Force by 20\% 

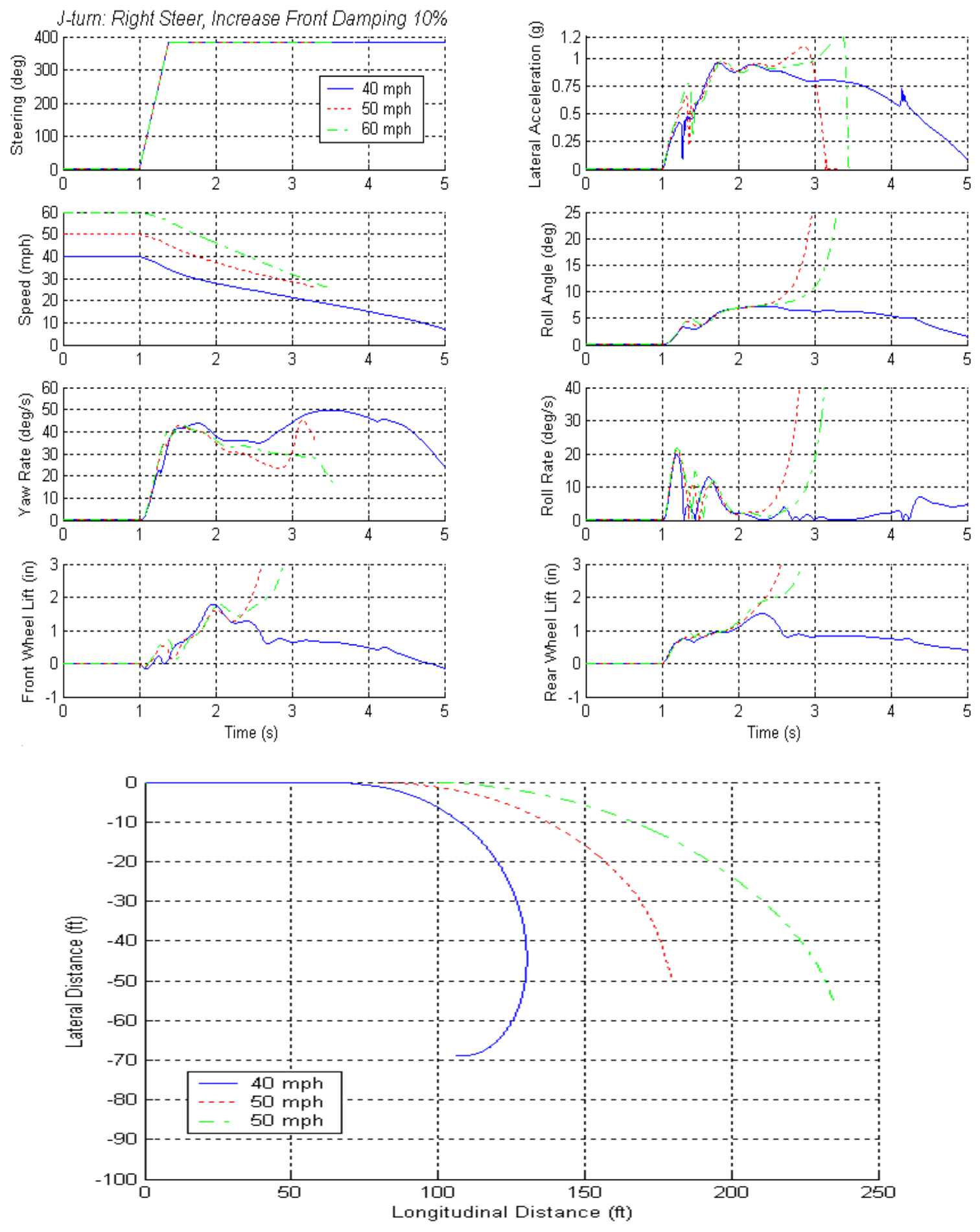

Figure 6.15: Shock Absorber Study, SLX Model "Left Side" Suspension with Lateral CG Offset Increase the Front Default Damping Force by 10\% 

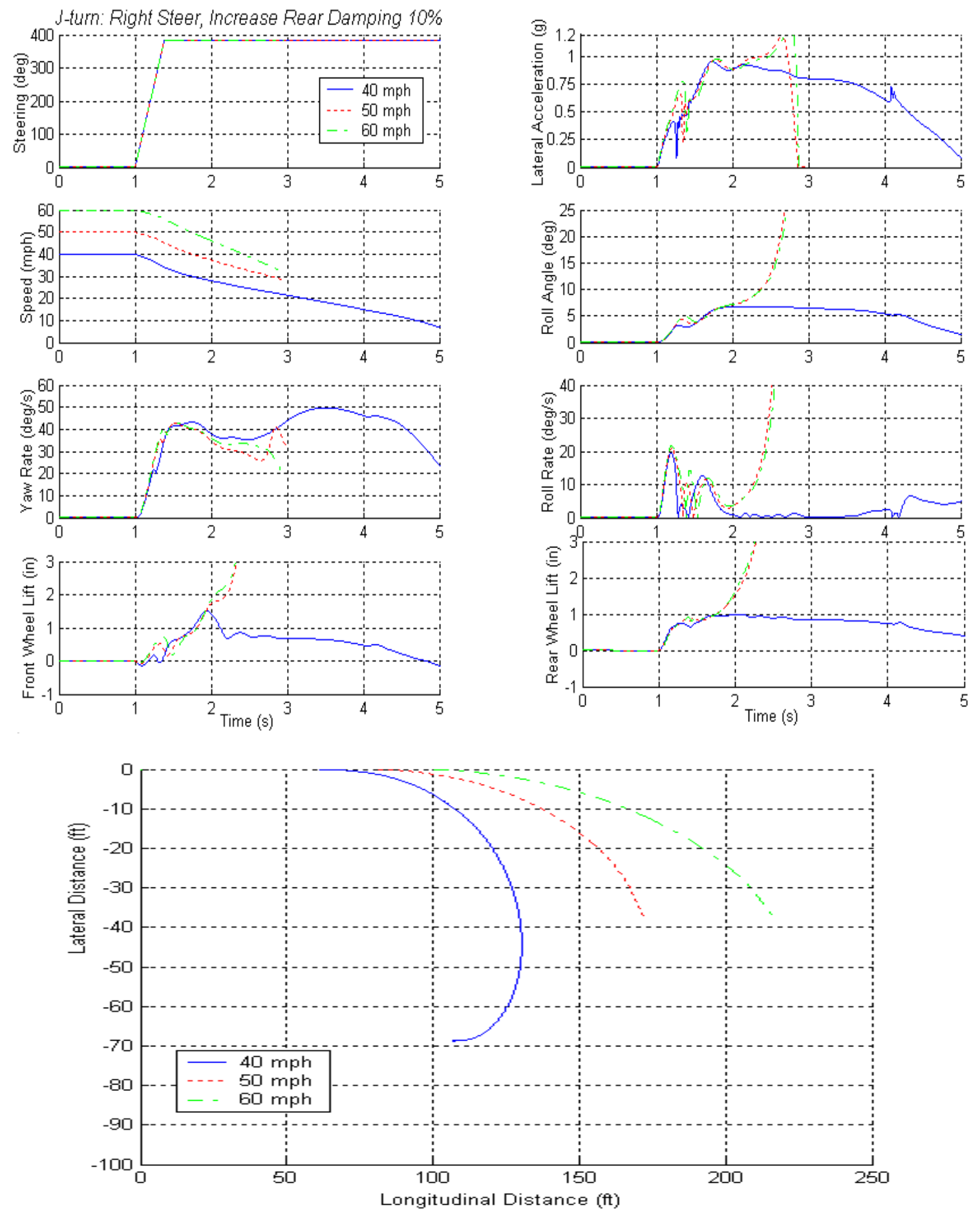

Figure 6.16: Shock Absorber Study, SLX Model "Left Side" Suspension with Lateral CG Offset Increase the Rear Default Damping Force by 10\% 


\section{CHAPTER 7}

\section{CONCLUSIONS AND RECOMMENDATIONS}

\subsection{Conclusions}

The main objective of this research was to study vehicle response asymmetries during severe driving maneuvers. During Phase VI of the National Highway Traffic Safety Administration’s (NHTSA) Light Vehicle Rollover Research Program, such response asymmetries occurred during field tests, and consequently, the data collected

during Phase VI was used in this study. Of the twenty-six light vehicles tested in Phase VI, three vehicles exhibited significant response asymmetries with respect to left versus right steer maneuvers: the 1996 Acura SLX, 1995 Mitsubishi Montero, and the 1993 Ford Aerostar

Ideally, the vehicle response should be the same given identical steering and speed inputs as well as surface and environmental conditions. Such is the case, assuming vehicle symmetry, with modeling and computer simulation. However in actual field tests, test-to-test variability can cause small response fluctuations. Even more so, when testing at a vehicle’s TWL threshold speed (the maneuver entrance speed for which twowheel lift may or may not occur), the vehicle response is highly nonlinear and to some extent unpredictable. The combination of test-to-test variability and testing at TWL 
threshold speeds adds to the complexity of analyzing vehicle response, e.g., when comparing left versus right steer vehicle response. Additional possible reasons for the asymmetric response were discussed in Chapter 4 and include the accuracy and repeatability of the collected data, common vehicle asymmetries, and vehicle understeer and roll gradients.

The cause of the vehicle asymmetric response is quite complex and cannot be narrowed down to one item alone, e.g., differences in the suspension spring rates. The vehicle responds to all of the inputs (vehicle speed, handwheel angle and rate, environmental conditions, test surface, tires, etc), so evaluating a single factor is difficult in real world testing. Careful consideration was given to possible unintended differences in the vehicle inputs that may cause vehicle asymmetric response. The unintended vehicle inputs evaluated include deviations in the desired commanded steering inputs, fluctuations of the environmental conditions, inconsistencies in the test surface, initial handwheel angle offset prior to the maneuver and any instrumentation offsets, and the effect of tire wear and worn/damaged suspension components as a result of testing. Aside from the tire and suspension wear, these unintended vehicle inputs were determined to be either negligible or to have an insignificant impact on the vehicle asymmetric response.

Given the severity of the tests, it is likely that significant tire and suspension wear occurred. Unfortunately, no measurements of tire wear (tread depth, tread shoulder wear and temperature, etc.) or suspension forces/deflections were made. It was determined that relative to the road edge recovery maneuver, the magnitude of these effects is less for the simpler, single steer, J-turn maneuver, and therefore, the J-turn maneuver was 
considered to be more reliable in indicating vehicle asymmetric response. In addition, modeling the dynamics of the J-turn and determining the effect of suspension and/or steering asymmetries is more intuitive for the J-turn.

An attempt was made to minimize the undesirable effect of tire and suspension wear in the Phase VI tests through frequent tire changes and suspension and body inspections. As such, the likely cause of the vehicle response asymmetries is within the vehicle itself and not attributed to the test procedure/conditions. Suspension and steering tests were conducted on the SLX and the Montero to identify any vehicle components/ characteristics, such as steering system or suspension asymmetries, that may cause vehicle asymmetric response.

The major suspension response asymmetries occurred in the bounce and roll tests. Both vehicles exhibited lower bump stop and roll (with respect to the top of the vehicle rolling to that side) stiffness values on the side of the suspension that was opposite to the more severe steer direction in the J-turn maneuver. For example, the SLX experiences TWL at a lower maneuver entrance speed in a left steer versus a right steer J-turn maneuver, and its right side suspension is softer than the left side. The same phenomenon applies to the Montero: it experiences TWL at a lower maneuver entrance speed in a right steer J-turn maneuver, and its left side suspension is softer.

Given the nonlinear suspension response upon bump stop contact, it is difficult to quantitatively compare the right and left side of the suspension response during extreme compression. However, linearized values were extracted for the initial bump stop contact and extreme bump stop compression. The linearized bump stop stiffness values were greater for the left side of the suspension by 20 to $40 \%$ for the front and 7 to $26 \%$ for the 
rear of the SLX. The Montero linearized bump stop stiffnesses were greater for the right side by 2 to $20 \%$ for the front and negligible for the rear. Based on linear regression analysis, the SLX front roll stiffness values were 3.1\% and $12.0 \%$ stiffer in counterclockwise body roll for the nominal load and maximum occupancy configuration, respectively. The Montero front roll stiffness values were $3.3 \%$ and $1.6 \%$ stiffer in clockwise body roll for the nominal load and maximum occupancy configuration, respectively. There were no noticeable differences in the rear roll stiffness values for either vehicle.

Of the physical suspension asymmetries observed during the suspension tests, the most significant was the attachment points of the Panhard rod used in the rear suspension of both the SLX and the Montero. The rod's configuration supports the rollover tendencies of both vehicles, but the magnitude of the effect is unknown.

To confirm the trend that the softer side suspension will lead to earlier TWL in the opposite steer direction, a model representative of the SLX was created and evaluated using the vehicle dynamics computer simulation, CarSim ${ }^{\mathrm{TM}}$. Based on the simulation study, the lateral CG offset has a significant effect on the vehicle response. This supports the rollover model in Chapter 3 and the idea of an "effective SSF". Interestingly, the lateral CG offset dominated the vehicle response. Using the actual suspension parameters (spring force versus deflection data, and auxiliary roll stiffness) for the SLX showed some differences between left and right steer response, but to a much lesser extent than the lateral CG offset. Given that, the bump stops may not have had an effect on the model. In the field tests, the SLX frequently experienced roll angles of $10^{\circ}$ without TWL and had an oscillatory roll response that is indicative of bump stop contact. 
It is unfortunate that the model seems to be missing that middle ground, i.e., either early level off of response or complete rollover. Although the simulation results show that the suspension asymmetric response has little effect on the vehicle response, the trend between the J-turn maneuver steer direction and the opposite side suspension relative stiffness cannot be ignored.

The response of the shock absorbers was not determined during the suspension and steering tests described in Chapter 5, but a simulation study was done to evaluate the effect of the shock absorbers on vehicle response. A series of simulation runs were preformed with the default damping force as is, decreased by $10 \%$, increased by $10 \%$, and increased by $20 \%$. Also a series of simulations was performed with only the front or rear damping force increased by $10 \%$. Based on the shock absorber study, it is evident that a change in the damping force response can have a significant effect on the vehicle response. As the default damping force was increased from $-10 \%$ to $+20 \%$, the vehicle model went from immediate rollover to delayed rollover to only TWL and finally to slight wheel lift. When compared to the rear increase only, the front increase only simulations (where only the front damping force was increased by 10\%) produced more wheel lift at $40 \mathrm{mph}$ but delayed rollover by almost half a second in the 50 and $60 \mathrm{mph}$ runs.

According to Dixon [29], "even when correctly assembled and free of such gross errors, dampers of the same design, and intended to have the same performance, are frequently found to have forces differing by $20 \%$ or more." As such, it is possible that the shocks used in the field tests were different with respect to the left versus right side of the vehicle. Furthermore, the findings of the shock absorber simulation study indicate 
that subtle changes to the front/rear force distribution can also have a significant effect on vehicle response. In addition to the shocks, seemingly identical tires can have tire-to-tire variations.

\subsection{Recommendations}

Based on the significant effect of tire wear discussed in Chapter 4, a new set of tires should be used to verify the minimum maneuver entrance speed for which TWL occurred, with the first test on the new tires being at that isolated speed. This was not the tire change protocol used in Phase VI, but according to a recent NHTSA document [19], future field tests performed by NHTSA will rerun the maneuver with new tires if the maneuver entrance speed that produced TWL was at least $47.5 \mathrm{mph}$. Upon installing new tires, the first test will be run at $35 \mathrm{mph}$, and if TWL does not occur, the next test will be at the maneuver entrance speed that produced TWL.

Although the 1993 Ford Aerostar did not exhibit as extreme response asymmetries as the SLX and the Montero during Phase VI testing, the Aerostar should undergo the same suspension and steering tests to further verify the trend between the Jturn steer direction and the opposite side suspension relative stiffness.

Further investigation of the validity of CarSim $^{\mathrm{TM}}$ in predicting vehicle rollover should be conducted as well as using other vehicle simulations such as ADAMS to test the effect of the SLX's and Montero's suspension asymmetric responses, including the effect of the Panhard rod as discussed in Section 5.4. In addition, the response of the shock absorbers on both the SLX and the Montero should be tested and incorporated into a representative model in CarSim ${ }^{\mathrm{TM}}$ or other simulation package to reevaluate the 
model's response with the actual shock absorber response in place of the CarSim ${ }^{\mathrm{TM}}$ default values used in the current research effort. 


\section{LIST OF REFERENCES}

1. Elsasser, D., Forkenbrock, G. J., O’Harra, B C., “An Experimental Examination of 26 Light Vehicles Using Test Maneuvers that May Induce On-Road, Untripped Rollover and a Discussion of NHTSA's Refined Test Procedures - Phases VI and VII of NHTSA’s Light Vehicle Rollover Research Program,” NHTSA Technical Report, DOT HS 809 547, October 2003.

2. Howe, J.G., Garrott, W.R., Forkenbrock, G. J., Heydinger, G., Lloyd, J., “An Experimental Examination of Selected Maneuvers That May Induce On-Road, Untripped, Light Vehicle Rollover - Phase I-A of NHTSA’s 1997-1998 Light Vehicle Rollover Research Program,” NHTSA Technical Report, DOT HS 809 357, August 2001.

3. Howe, J.G., Garrott, W.R., Forkenbrock, G. J., Lloyd, J., “An Experimental Examination of Selected Maneuvers That May Induce On-Road, Untripped, Light Vehicle Rollover - Phase I-B of NHTSA's 1997-1998 Light Vehicle Rollover Research Program,” NHTSA Technical Report, DOT HS 809 443, April 2002.

4. Howe, J.G., Garrott, W.R., Forkenbrock, “An Experimental Examination of Selected Maneuvers That May Induce On-Road, Untripped, Light Vehicle Rollover - Phase II of NHTSA’s 1997-1998 Light Vehicle Rollover Research Program,” NHTSA Technical Report, DOT HS 808 977, July 1999.

5. Forkenbrock, G. J., “Automated Steering Reversals Performed at Maximum Roll Angle in Fishhook Maneuver - Phase III-A of NHTSA's Light Vehicle Rollover Research Program,” NHTSA Technical Report (number not yet available), in press.

6. Forkenbrock, G. J., "Automated Pulse Braking in a J-turn Maneuver - Phase III-B of NHTSA’s Light Vehicle Rollover Research Program,” NHTSA Technical Report (number not yet available), in press. 
7. Forkenbrock, G. J., Garrott, W. R, Heitz, M., O’Harra, B. C., “A Comprehensive Experimental Examination of Test Maneuvers That May Induce On-Road, Untripped Light Vehicle Rollover - Phase IV of NHTSA's Light Vehicle Rollover Research Program,” NHTSA Technical Report, DOT HS 809 513, October 2002.

8. "Traffic Safety Facts 2001: A Compilation of Motor Vehicle Crash Data from the Fatality Analysis Reporting System and the General Estimates System,” NHTSA Report, DOT HS 809 484, December 2002.

9. “2002 Annual Assessment - Motor Vehicle Traffic Crash Fatality and Injury Estimates for 2002,” NHTSA Report, July 2003.

10. SAE J266, Surface Vehicle Recommended Practice, "Steady-State Directional Control Test Procedures For Passenger Cars and Light Trucks,” 1996.

11. Chrstos, J. P., Guenther, D. A., “The Measurement of Static Rollover Metrics,” SAE Paper No. 920582, 1992.

12. "DOT Announces Proposal to Add Rollover Ratings To Auto Safety Consumer Information Program,” NHTSA NOW, Vol. 6, No. 7, June 19, 2000.

13. Gillespie, T. D., Fundamentals of Vehicle Dynamics, Society of Automotive Engineers, 1992.

14. Bernard, J., Shannan, J., and Vanderploeg, M., "Vehicle Rollover on Smooth Surfaces,” SAE paper No. 891991, 1989.

15. Heydinger, G., and Howe, J. G., "Analysis of Vehicle Response Data Measured During Severe Maneuvers,” SAE paper No. 2000-01-1644, 2000.

16. Hac, A., "Rollover Stability Index Including Effects of Suspension Design," SAE paper No. 2002-01-0965, 2002.

17. Liu, L. Y., Yang, H., “A Robust Active Suspension Controller with Rollover Prevention,” SAE paper No. 2003-01-0959, 2003. 
18. Allen, R. W., Szostak, H. T., Rosenthal, T. J., Klyde, D. H., and Owens, K. J., "Vehicle Dynamic Stability and Rollover," Technical Report No. 1268-1, Systems Technology, Inc., June 1992.

19. Docket No. NHTSA-2001- 9663; Notice 3 (\& 2), RIN 2127-AI81, Federal Register, Vol. 68, No. TBD, October 07, 2003.

20. “Assessing the Feasibility of Using ASTM E1136 Standard Reference Test Tires During Road Edge Recovery Maneuvers,” NHTSA Report (number not yet available), in press.

21. “Tire Shoulder Wear in Repetitive Rollover Testing,” Report No. 111901, Automotive Testing, Inc., no published date given.

22. Milliken, W. F. \& D. L., Race Car Vehicle Dynamics, Society of Automotive Engineers, 1995.

23. "Vehicle Dynamics Terminology,” SAE Recommended Practice, SAE Report J670e, July 1976.

24. Jordan, M., “The \$30,000 Personality Test,” Automobile Magazine, October 2003.

25. Runge, J. W., and Weinstein, K. N., “Firestone’s Request for a Defect Investigation on the Handling of Ford Explorers After a Rear Tire Tread Separation,” NHTSA, 2002.

26. “On Demand Repair”, Mitchell Repair Information Company, 2003.

27. Mechanical Simulation Corporation (MSC) Home Page, available at: http://www.carsim.com October 2003.

28. Salaani, M., Heydinger, G., Guenther, D., "Vehicle Dynamics Modeling for the National Advanced Driving Simulator of a 1997 Jeep Cherokee,” SAE paper No. 1999-01-0121, 1999. 
29. Dixon, J.C., The Shock Absorber Handbook, Society of Automotive Engineers, 1999. 


\title{
APPENDIX \\ MISCELLANEOUS EQUATIONS AND CARSIM ${ }^{\mathrm{TM}}$ PARSFILE
}

\author{
Matlab ${ }^{\circledR}$ Code that determines the sprung mass inertias based on the total vehicle inertias \\ and the CG locations.
}

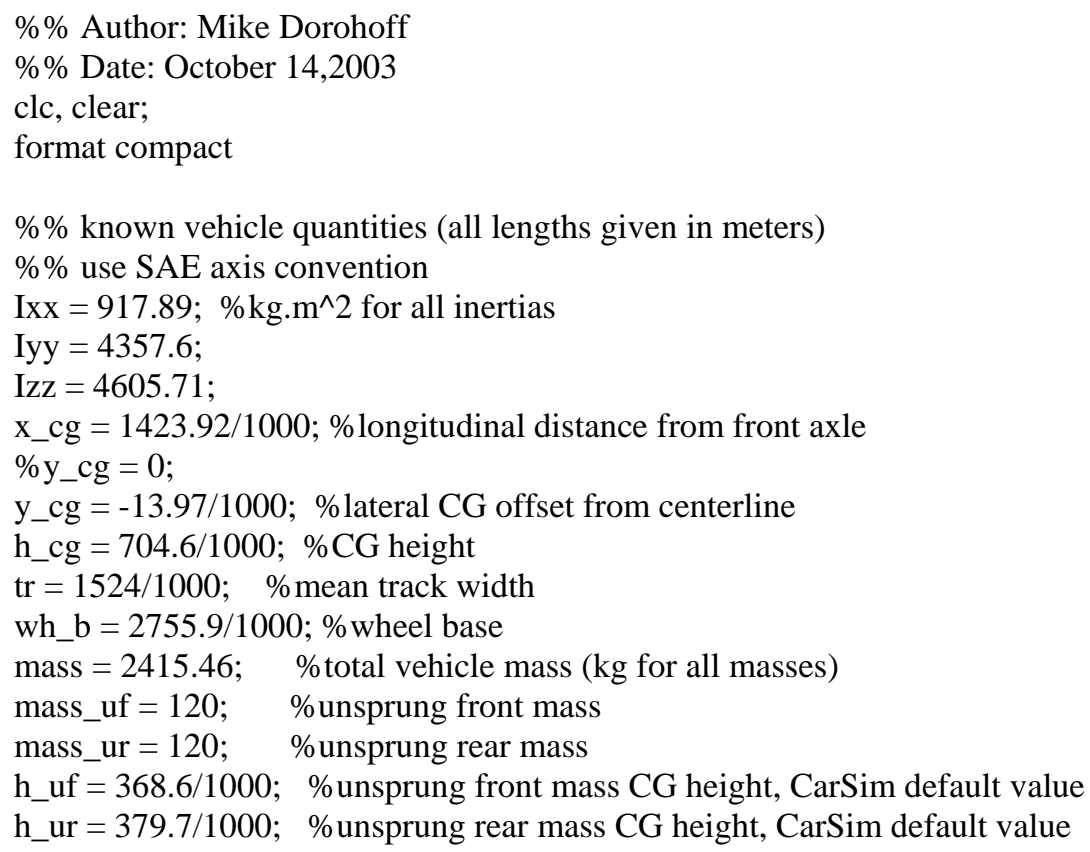

$\% \%$ calculate the sprung mass CG locations based on static moment equation

$\%$ The CG locations of the unsprung masses were assumed to be at the loaded wheel

$\%$ centers (i.e., four separate masses represent the unsprung masses). The

$\%$ lateral CG distance between the total vehicle CG and the front and rear

$\%$ unsprung masses were assumed to be negligible. See the end of this

$\%$ code for verification of this.

mass_s = mass - mass_uf - mass_ur; \%sprung mass

\%sprung mass, longitudinal distance from the front axle

x_scg $=($ mass*x_cg - mass_uf*0 - mass_ur*wh_b)/mass_s

\%lateral sprung mass CG offset (negative toward driver side)

y_scg $=$ (mass*y_cg)/mass_s 


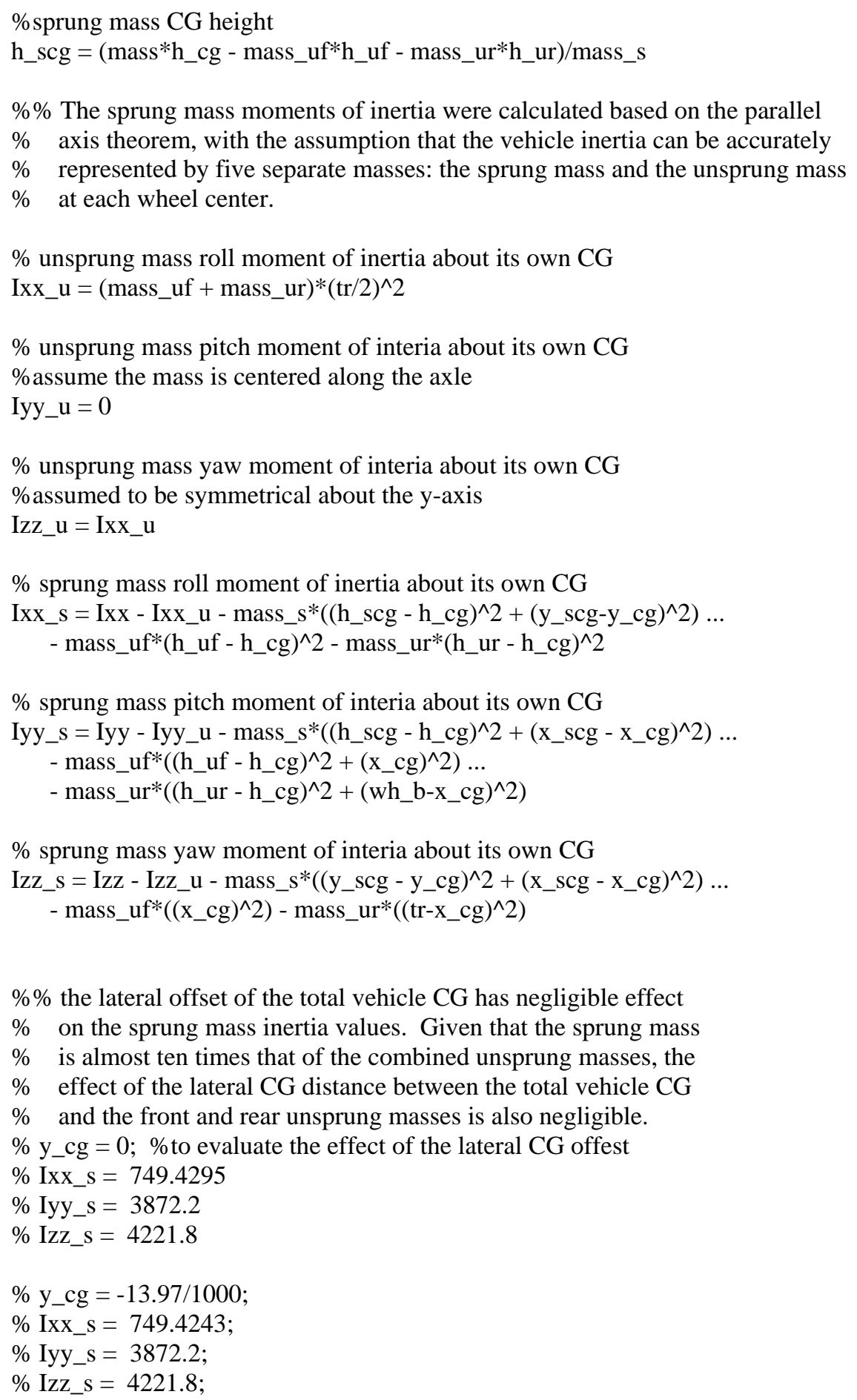




\section{CarSim ${ }^{\mathrm{TM}}$ Parsfile for a particular simulation run with SLX Model (parts deemed unnecessary, e.g. animator information, were deleted)}

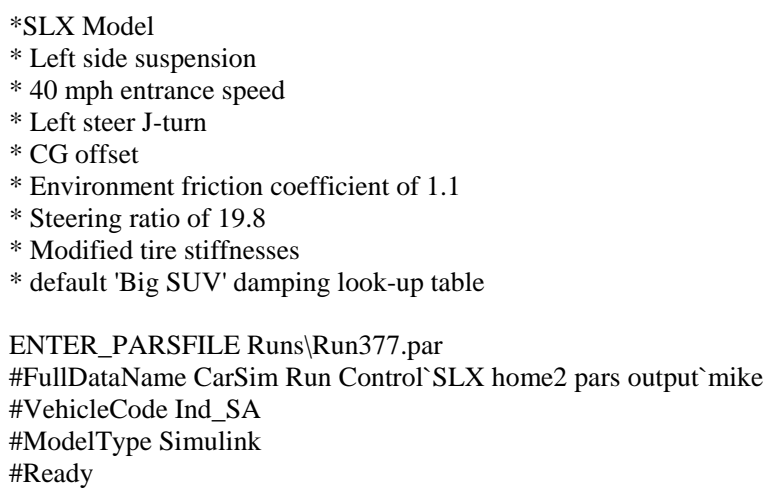


*RSW F 19.8

*RSW_R 20.0

*VLOW_STR_COMP(1) 1.5

*VLOW_STR_COMP(2) 1.5

*L1_LKPO 100

*L2_LKPO 100

*R1_LKPO 100

*R2_LKPO 100

*L1_AKPI 12

*L2_AKPI 8

*R1_AKPI 12

*R2 AKPI 8

*L1_ACAST 2

*L2_ACAST 1

*R1_ACAST 2

*R2_ACASTI 1

*L1_XKPO 0

*L2_XKPO 0

*R1_XKPO 0

*R2_XKPO 0

\#Ready

ENTER_PARSFILE Steering \Rear_Steer|RearStr101.par

\#FullDataName Steering System: Rear-Wheel Gain `No Rear Steering`

\#DiagramOne0

R_STEER_SPEED_TABLE 2

$0.000000,0.000000$

5.000000, 0.000000

ENDTABLE

Title No Rear Steering

\#DataSet : No Rear Steering

\#Category:

\#FileID : RearStr101

\#Product : CarSim Version 5.15 October 2002

\#Last update: 10-28-2002 12:10:05

\#LinkCat Steer Gain vs. Speed

EXIT_PARSFILE Steering|Rear_Steer|RearStr101.par

\#BlueLink6, Steering System: Rear-Wheel Gain`No Rear Steering ` , Rear steering gain vs. speed \#Ready

ENTER_PARSFILE Steering|Str_Wheel_Tq\TqSW107.par

\#FullDataName Steering Wheel Torque ${ }^{\circ} 1 / 20 `$

*3D_XLabel Speed $(\mathrm{km} / \mathrm{h})$

*3D_YLabel Total kingpin moment $(\mathrm{N}-\mathrm{m})$

*3D_ZLabel Steering-wheel torque (N-m)

\#DiagramTwo

*3D_DATA 2, 2 --> columns $x$ rows

M_SW_CARPET 3

$0,0.0,200.0$

$0.0,0.0,0.0$

20.0, 1.0, 1.0

ENDTABLE

Title $1 / 20$

\#DataSet : $1 / 20$

\#Category:

\#FileID : TqSW107

\#Product : CarSim Version 5.15 October 2002

\#Last update: 10-28-2002 12:10:04

\#LinkCat Steering torque

EXIT_PARSFILE Steering|Str_Wheel_Tq\TqSW107.par

\#BlueLink7, Steering Wheel Torque`1/20` , Rear steering gain vs. speed

iaxle 1

iside 1

R_GEAR_STR 19.8

VLOW_STR_COMP 1.5

\#Ready 
ENTER_PARSFILE Steering \CompliancelStrCmp100.par

\#FullDataName Steering System: Compliance`SUV - 0.002 deg/N-m`

\#DiagramOne0

STEER_COMP_TABLE 2

$0.000000,0.000000$

1.000000, 0.000500

ENDTABLE

Title SUV - $0.002 \mathrm{deg} / \mathrm{N}-\mathrm{m}$

\#DataSet : SUV - $0.002 \mathrm{deg} / \mathrm{N}-\mathrm{m}$

\#Category:

\#FileID : StrCmp100

\#Product : CarSim Version 5.15 October 2002

\#Last update: 10-28-2002 12:10:06

\#LinkCat Steering compliance

EXIT_PARSFILE Steering\Compliance\StrCmp100.par

\#BlueLink4, Steering System: Compliance`SUV - 0.002 deg/N-m` `, F steering system compliance \#Ready

ENTER_PARSFILE Steering\Kinematics\StrKin101.par

\#FullDataName Steering System: Kinematics for One Wheel`Example Left Wheel Data`

\#DiagramOne0

STEER_KIN_TABLE 2

$-22.670000,-21.460000$

$-19.830000,-18.910000$

$-17.000000,-16.320000$

$-14.170000,-13.700000$

$-11.330000,-11.030000$

$-8.500000,-8.330000$

$-5.670000,-5.590000$

$-2.830000,-2.810000$

$0.000000,0.000000$

$2.830000,2.850000$

$5.670000,5.740000$

$8.500000,8.670000$

$11.330000,11.630000$

$14.170000,14.640000$

$17.000000,17.680000$

$19.830000,20.750000$

22.670000, 23.870000

ENDTABLE

Title Example Left Wheel Data

\#DataSet : Example Left Wheel Data

\#Category:

\#FileID : StrKin101

\#Product : CarSim Version 5.15 October 2002

\#Last update: 10-28-2002 12:10:05

\#LinkCat Steering kinematics

EXIT_PARSFILE Steering\Kinematics\StrKin101.par

\#BlueLink0, Steering System: Kinematics for One Wheel`Example Left Wheel Data` ’, L1 steering kinematics L_KPO 100

A_KPI 12

A_CASTER 2

X_KPO 0

iaxle 2

iside 1

R_GEAR_STR 20.0

VLOW_STR_COMP 1.5

\#Ready

ENTER_PARSFILE Steering\CompliancelStrCmp100.par

\#FullDataName Steering System: Compliance `SUV - 0.002 deg/N-m`

\#DiagramOne0

STEER_COMP_TABLE 2

$0.000000,0.000000$

$1.000000,0.000500$

ENDTABLE

Title SUV - $0.002 \mathrm{deg} / \mathrm{N}-\mathrm{m}$ 


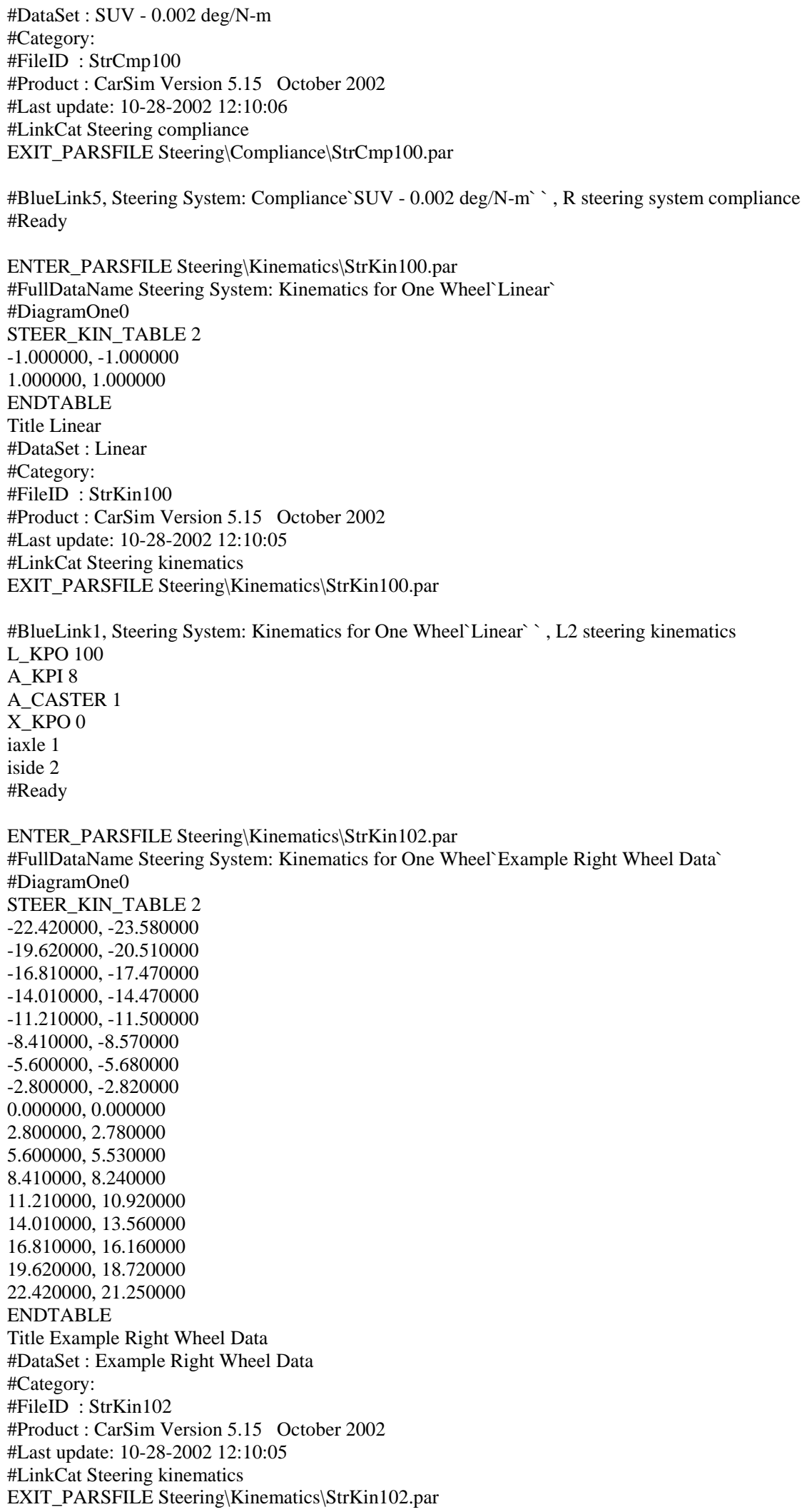




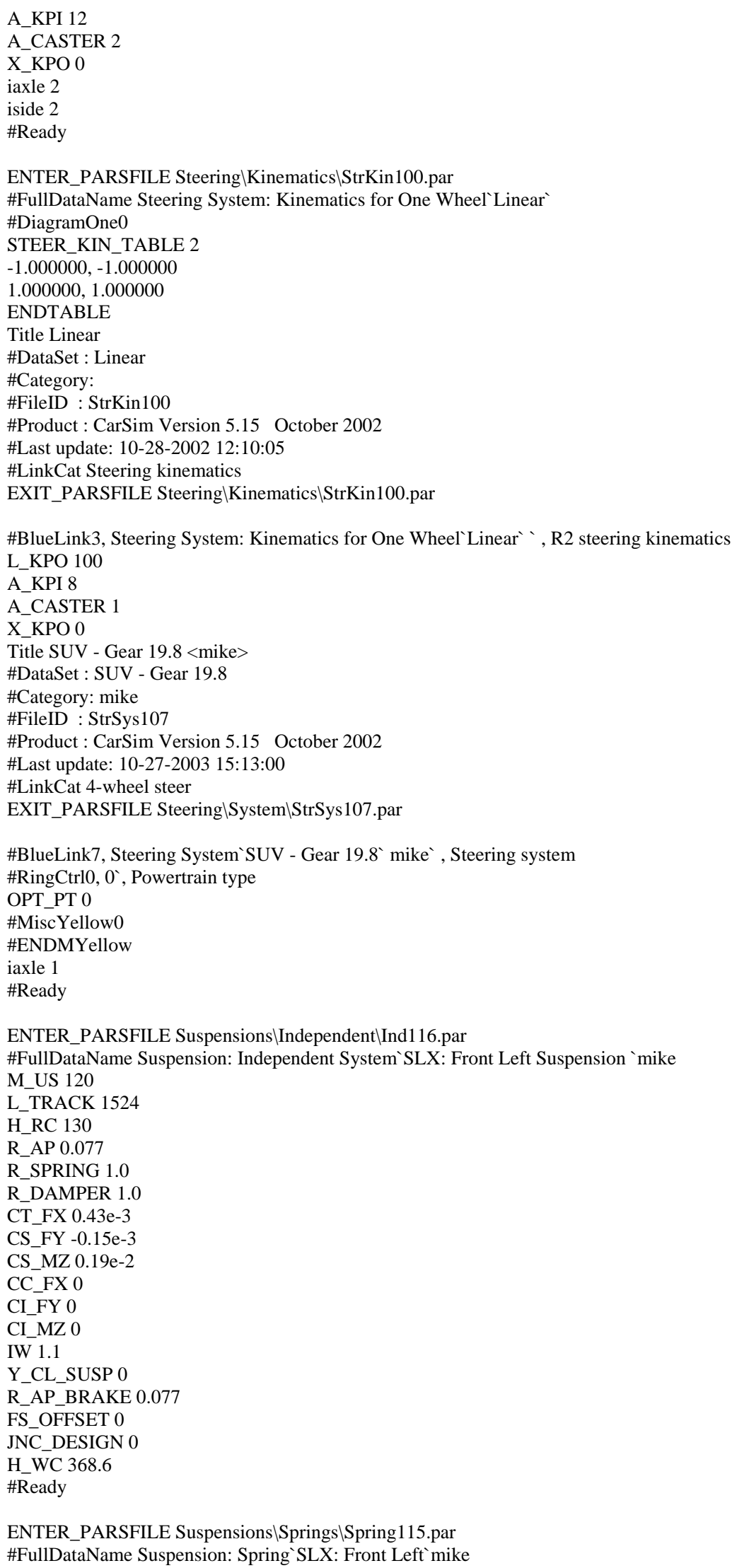


SPRING_COMP_BETA 2

SPRING_EXT_BETA 2

\#DiagramOne0

FS_COMP_TABLE 2

$-120.570000,-607.870000$

$-116.140000,488.390000$

$-110.090000,1019.240000$

$-99.870000,1396.000000$

$-88.630000,1761.640000$

$-78.100000,2099.840000$

$-66.750000,2430.030000$

$-55.290000,2781.880000$

$-44.550000,3107.760000$

$-33.060000,3434.610000$

$-21.650000,3683.350000$

$-9.730000,4058.320000$

$0.000000,4500.000000$

$11.150000,4853.270000$

23.480000, 5129.330000

$36.640000,5566.810000$

44.860000, 6321.450000

$52.460000,7303.430000$

59.280000, 8468.910000

65.380000, 9848.960000

69.960000, 11516.590000

ENDTABLE

\#DiagramOne1

FS_EXT_TABLE 2

$-120.570000,-807.870000$

$-116.140000,288.390000$

$-110.090000,819.240000$

$-99.870000,1196.000000$

$-88.630000,1561.640000$

$-78.100000,1899.840000$

$-66.750000,2230.030000$

$-55.290000,2581.880000$

$-44.550000,2907.760000$

$-33.060000,3234.610000$

$-21.650000,3483.350000$

$-9.730000,3858.320000$

$0.000000,4300.000000$

$11.150000,4653.270000$

23.480000, 4929.330000

$36.640000,5366.810000$

$44.860000,6121.450000$

$52.460000,7103.430000$

$59.280000,8268.910000$

$65.380000,9648.960000$

$69.960000,11316.590000$

ENDTABLE

Title SLX: Front Left <mike>

\#DataSet: SLX: Front Left

\#Category: mike

\#FileID : Spring115

\#Product : CarSim Version 5.15 October 2002

\#Last update: 10-27-2003 15:16:32

\#LinkCat Spring

EXIT_PARSFILE Suspensions\Springs\Spring115.par

\#BlueLink0, Suspension: Spring`SLX: Front Left`mike`, Spring \#Ready

ENTER_PARSFILE Suspensions\Shocks\Shock106.par \#FullDataName Suspension: Shock Absorber`Big SUV Damping \#DiagramOne0

FD_TABLE 2

$-1410.000000,-7316.000000$

$-720.000000,-5019.000000$

$-390.000000,-3395.000000$ 
$-210.000000,-2618.000000$

$-90.000000,-1472.000000$

$-20.000000,-333.000000$

20.000000, 333.000000

$90.000000,870.000000$

$200.000000,1145.000000$

$390.000000,1607.000000$

$760.000000,2623.000000$

$1160.000000,3740.000000$

ENDTABLE

Title Big SUV Damping

\#DataSet : Big SUV Damping

\#Category:

\#FileID : Shock106

\#Product : CarSim Version 5.15 October 2002

\#Last update: 10-28-2002 12:10:00

\#LinkCat Shock absorber

EXIT_PARSFILE Suspensions\ShocksıShock106.par

\#BlueLink1, Suspension: Shock Absorber`Big SUV Damping` ’, Damper \#Ready

ENTER_PARSFILE Suspensions\ToelToe104.par

\#FullDataName Suspension: Toe Angle`Example Front Toe`

\#DiagramOne0

TOE_TABLE 2

$-70.000000,1.060000$

$-60.000000,0.830000$

$-50.000000,0.610000$

$-40.000000,0.430000$

$-30.000000,0.270000$

$-20.000000,0.140000$

$-10.000000,0.030000$

$0.000000,-0.050000$

$10.000000,-0.100000$

$20.000000,-0.130000$

$30.000000,-0.140000$

$40.000000,-0.110000$

$50.000000,-0.060000$

$60.000000,0.010000$

$70.000000,0.120000$

ENDTABLE

Title Example Front Toe

\#DataSet : Example Front Toe

\#Category:

\#FileID : Toe104

\#Product : CarSim Version 5.15 October 2002

\#Last update: 10-28-2002 12:09:58

\#LinkCat Toe

EXIT_PARSFILE Suspensions\Toe\Toe104.par

\#BlueLink2, Suspension: Toe Angle`Example Front Toe``, Toe vs. Jounce \#Ready

ENTER_PARSFILE Suspensions\CamberlCamber101.par

\#FullDataName Suspension: Camber Angle`Example Front Camber`

\#DiagramOne0

CAMBER TABLE 2

$-70.000000,0.430000$

$-60.000000,0.440000$

$-50.000000,0.420000$

$-40.000000,0.380000$

$-30.000000,0.310000$

$-20.000000,0.230000$

$-10.000000,0.130000$

$0.000000,0.000000$

$10.000000,-0.150000$

$20.000000,-0.320000$

$30.000000,-0.510000$ 


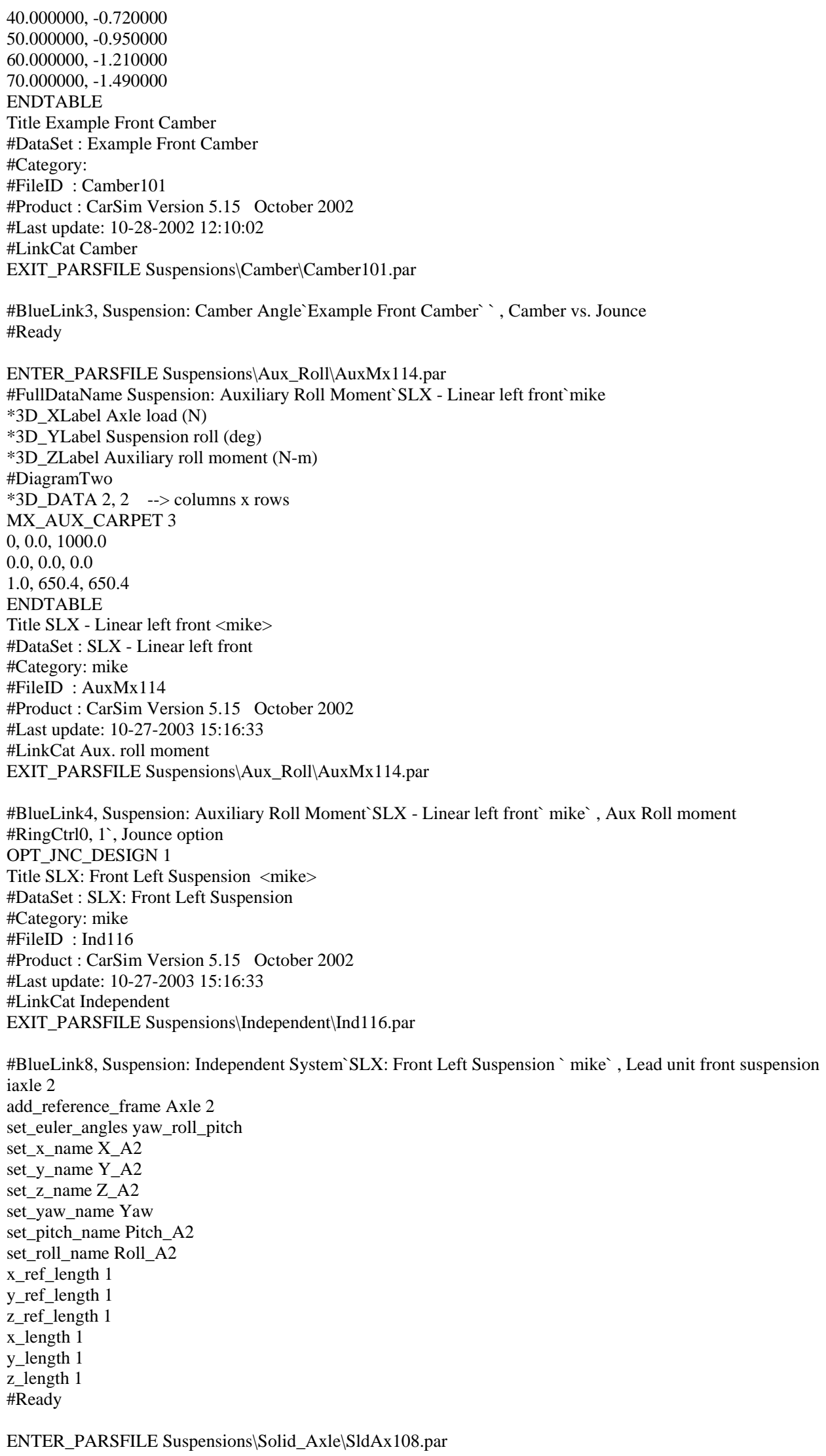


\#FullDataName Suspension: Solid Axle System `SLX: Rear Left Suspension `mike

H_RC 350

L_TRACK 1473.2

R_AP -0.1

L_SPRINGS 868.7

L DAMPERS 868.7

R_SPRING 1.0

R_DAMPER 1.0

CT_FX 0

CS FY $-0.56 \mathrm{e}-5$

CS_MZ 0.95e-3

CC_FX 0

CI_FY 0

CI_MZ 0

M_US 120

IA 40

IW 1.1

R_ROLL_STEER 0

Y_CL_SUSP 0

*TOE_L 0

*TOE_R 0

*CAMBER_L 0

*CAMBER_R 0

R_AP_BRAKE -0.1

FS_OFFSET 0

H_WC 379.7

H_CG_AXLE 379.7

\#Ready

ENTER_PARSFILE Suspensions\Springs\Spring116.par \#FullDataName Suspension: Spring 'SLX: Rear Left ’mike SPRING_COMP_BETA 2

SPRING_EXT_BETA 2

\#DiagramOne0

FS_COMP_TABLE 2

$-126.400000,-185.180000$

$-123.680000,776.470000$

$-115.500000,1421.860000$

$-104.660000,1731.190000$

$-95.320000,1999.110000$

$-84.510000,2310.350000$

$-73.700000,2621.720000$

$-63.110000,2956.800000$

$-52.620000,3266.400000$

$-41.920000,3553.970000$

$-31.040000,3892.620000$

$-21.020000,4231.880000$

$-10.890000,4502.900000$

$-1.020000,4842.760000$

$0.000000,4900.000000$

8.620000, 5515.940000

$16.860000,6138.960000$

25.840000, 6695.690000

34.020000, 7402.110000

41.200000, 8235.570000

46.310000, 9355.320000

$50.510000,10705.790000$

53.340000, 12146.120000

ENDTABLE

\#DiagramOne1

FS EXT TABLE 2

$-126.400000,-385.180000$

$-123.680000,576.470000$

$-115.500000,1221.860000$

$-104.660000,1531.190000$

$-95.320000,1799.110000$

$-84.510000,2110.350000$

$-73.700000,2421.720000$

$-63.110000,2756.800000$ 
$-52.620000,3066.400000$

$-41.920000,3353.970000$

$-31.040000,3692.620000$

$-21.020000,4031.880000$

$-10.890000,4302.900000$

$-1.020000,4642.760000$

$0.000000,4700.000000$

$8.620000,5315.940000$

$16.860000,5938.960000$

$25.840000,6495.690000$

$34.020000,7202.110000$

$41.200000,8035.570000$

46.310000, 9155.320000

$50.510000,10505.790000$

53.340000, 11946.120000

ENDTABLE

Title SLX: Rear Left <mike>

\#DataSet : SLX: Rear Left

\#Category: mike

\#FileID : Spring116

\#Product : CarSim Version 5.15 October 2002

\#Last update: 10-27-2003 15:16:34

\#LinkCat Spring

EXIT_PARSFILE Suspensions\Springs\Spring116.par

\#BlueLink0, Suspension: Spring`SLX: Rear Left` mike`, Spring

\#Ready

ENTER_PARSFILE Suspensions\Shocks\Shock106.par

\#FullDataName Suspension: Shock Absorber`Big SUV Damping

\#DiagramOne0

FD_TABLE 2

$-1410.000000,-7316.000000$

$-720.000000,-5019.000000$

$-390.000000,-3395.000000$

$-210.000000,-2618.000000$

$-90.000000,-1472.000000$

$-20.000000,-333.000000$

$20.000000,333.000000$

$90.000000,870.000000$

$200.000000,1145.000000$

$390.000000,1607.000000$

$760.000000,2623.000000$

$1160.000000,3740.000000$

ENDTABLE

Title Big SUV Damping

\#DataSet : Big SUV Damping

\#Category:

\#FileID : Shock106

\#Product : CarSim Version 5.15 October 2002

\#Last update: 10-28-2002 12:10:00

\#LinkCat Shock absorber

EXIT_PARSFILE SuspensionsiShocksıShock106.par

\#BlueLink1, Suspension: Shock Absorber`Big SUV Damping` ’, Shock

\#Ready

ENTER_PARSFILE Suspensions\Aux_Roll\AuxMx115.par

\#FullDataName Suspension: Auxiliary Roll Moment `SLX: Linear Rear Left `mike

*3D_XLabel Axle load (N)

*3D_YLabel Suspension roll (deg)

*3D_ZLabel Auxiliary roll moment (N-m)

\#DiagramTwo

*3D_DATA 2, 2 --> columns x rows

MX_AUX_CARPET 3

$0,0.0,1000.0$

$0.0,0.0,0.0$

1.0, 526.6, 526.6

ENDTABLE 
Title SLX: Linear Rear Left <mike>

\#DataSet : SLX: Linear Rear Left

\#Category: mike

\#FileID : AuxMx115

\#Product : CarSim Version 5.15 October 2002

\#Last update: 10-27-2003 15:16:34

\#LinkCat Aux. roll moment

EXIT_PARSFILE Suspensions\Aux_Roll\AuxMx115.par

\#BlueLink2, Suspension: Auxiliary Roll Moment`SLX: Linear Rear Left`mike`, Aux Mx \#Ready

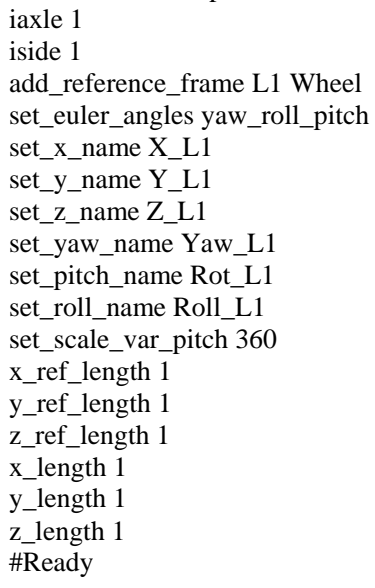


$0,2500.0,4100.0,5800.0$

$1.2,16.0,36.9,61.5$

$2.8,25.0,62.9,109.9$

$4.3,22.1,60.8,112.1$

$5.8,16.4,48.8,94.5$

$7.2,11.5,36.4,73.3$

8.9, 7.9, 26.0, 53.9

$10.4,5.6,19.0,40.4$

$11.8,4.1,14.1,30.4$

13.3, 3.0, 10.6, 23.2

$15.0,2.2,8.0,17.5$

$16.3,1.7,6.3,14.0$

$27.6,0.0,0.0,0.0$

28.8, $0.0,0.0,0.0$

ENDTABLE

Title Peak 110N-m, Load 5.8KN

\#DataSet : Peak 110N-m, Load 5.8KN

\#Category:

\#FileID : TireMz104

\#Product : CarSim Version 5.15 October 2002

\#Last update: 10-28-2002 12:09:55

\#LinkCat Tire: $\mathrm{Mz}$

EXIT_PARSFILE Tires\Mz\TireMz104.par

\#BlueLink0, Tire: Aligning Moment`Peak 110N-m, Load 5.8KN` `, Mz \#Ready

ENTER_PARSFILE Tires\Fy\TireFy104.par

\#FullDataName Tire: Lateral Force `Slope 0.17, Load 6.7KN`

MU_REF_Y 0.87

*3D_XLabel Vertical Load (N)

*3D_YLabel Slip Angle [Alpha] (deg)

*3D_ZLabel Absolute Lateral Tire Force (N)

\#DiagramTwo

*3D_DATA 3, 12 --> columns x rows

FY_TIRE_CARPET 4

0, 2900.0, 4800.0, 6700.0

$1.2,993.3,1344.6,1579.6$

2.9, 2110.3, 2966.7, 3559.0

4.4, 2648.6, 3850.3, 4717.3

6.0, 2888.7, 4296.6, 5346.2

7.6, 2979.9, 4497.3, 5654.9

9.2, 2998.2, 4570.0, 5788.8

$10.8,2979.1,4567.7,5812.2$

$12.4,2942.7,4529.6,5781.7$

$14.0,2897.7,4472.1,5720.7$

15.6, 2844.2, 4398.3, 5635.3

$17.0,2796.6,4329.8,5553.1$

20.0, 2796.6, 4329.8, 5553.1

ENDTABLE

Title Slope 0.17, Load 6.7KN

\#DataSet : Slope 0.17, Load 6.7KN

\#Category:

\#FileID : TireFy104

\#Product : CarSim Version 5.15 October 2002

\#Last update: 10-28-2002 12:09:56

\#LinkCat Tire: Fy

EXIT_PARSFILE Tires\Fy\TireFy104.par

\#BlueLink1, Tire: Lateral Force`Slope 0.17, Load 6.7KN` `, Fy

\#Ready

ENTER_PARSFILE Tires\Fx\TireFx103.par

\#FullDataName Tire: Longitudinal Force Peak 20\% Slip, Load 6.7KN` MU_REF_X 0.89

*3D_XLabel Vertical Load (N)

*3D_YLabel Abs. Slip Ratio [Kappa] (-)

*3D_ZLabel Absolute Longitudinal Tire Force (N)

\#DiagramTwo 
*3D_DATA 3, 12 --> columns x rows

FX_TIRE_CARPET 4

$0,2900.0,4800.0,6700.0$

$0.009,335.625,559.562,785.36$

$0.013,530.107,883.918,1240.425$

$0.027,1094.347,1815.034,2527.206$

$0.067,2326.209,3736.741,4998.142$

$0.133,2930.522,4581.301,5944.776$

0.2 , 2998.326, 4648.622, 5979.575

$0.267,2961.147,4576.08,5866.472$

$0.333,2900.795,4475.8,5728.702$

$0.4,2836.935,4373.485,5592.818$

$0.467,2775.123,4275.93,5465.168$

$0.533,2716.933,4184.816,5346.879$

0.6, 2662.641, 4100.222, 5237.549

ENDTABLE

Title Peak 20\% Slip, Load 6.7KN

\#DataSet : Peak 20\% Slip, Load 6.7KN

\#Category:

\#FileID : TireFx103

\#Product : CarSim Version 5.15 October 2002

\#Last update: 10-28-2002 12:09:57

\#LinkCat Tire: Fx

EXIT_PARSFILE Tires\Fx\TireFx103.par

\#BlueLink2, Tire: Longitudinal Force`Peak 20\% Slip, Load 6.7KN``, Fx

\#Ready

ENTER_PARSFILE Tires\Camber_Thrust\CamStiff101.par

\#FullDataName Tire: Camber Thrust Coefficient`Constant @ -60

\#DiagramOne0

KGAMMA_TABLE 2

$0.000000,-60.000000$

5000.000000, -60.000000

ENDTABLE

Title Constant @ -60

\#DataSet : Constant @ -60

\#Category:

\#FileID : CamStiff101

\#Product : CarSim Version 5.15 October 2002

\#Last update: 10-28-2002 12:09:55

\#LinkCat Camber thrust

EXIT_PARSFILE Tires\Camber_Thrust\CamStiff101.par

\#BlueLink3, Tire: Camber Thrust Coefficient`Constant @ -60` , Camber thrust \#BlueLink5, “' , External

Title SLX front 275/70R16 <mike>

\#DataSet : SLX front 275/70R16

\#Category: mike

\#FileID : Tire111

\#Product : CarSim Version 5.15 October 2002

\#Last update: 10-27-2003 15:13:04

\#LinkCat MSC model

EXIT_PARSFILE Tires\MSC_Model\Tire111.par

\#BlueLink9, Tire: MSC Model`SLX front 275/70R16` mike`, Lead unit left front tire iside 2

add_reference_frame R1 Wheel

set_euler_angles yaw_roll_pitch

set_x_name X_R1

set_y_name Y_R1

set_z_name Z_R1

set_yaw_name Yaw_R1

set_pitch_name Rot_R1

set_roll_name Roll_R1

set_scale_var_pitch 360

x_ref_length 1

y_ref_length 1

z_ref_length 1 


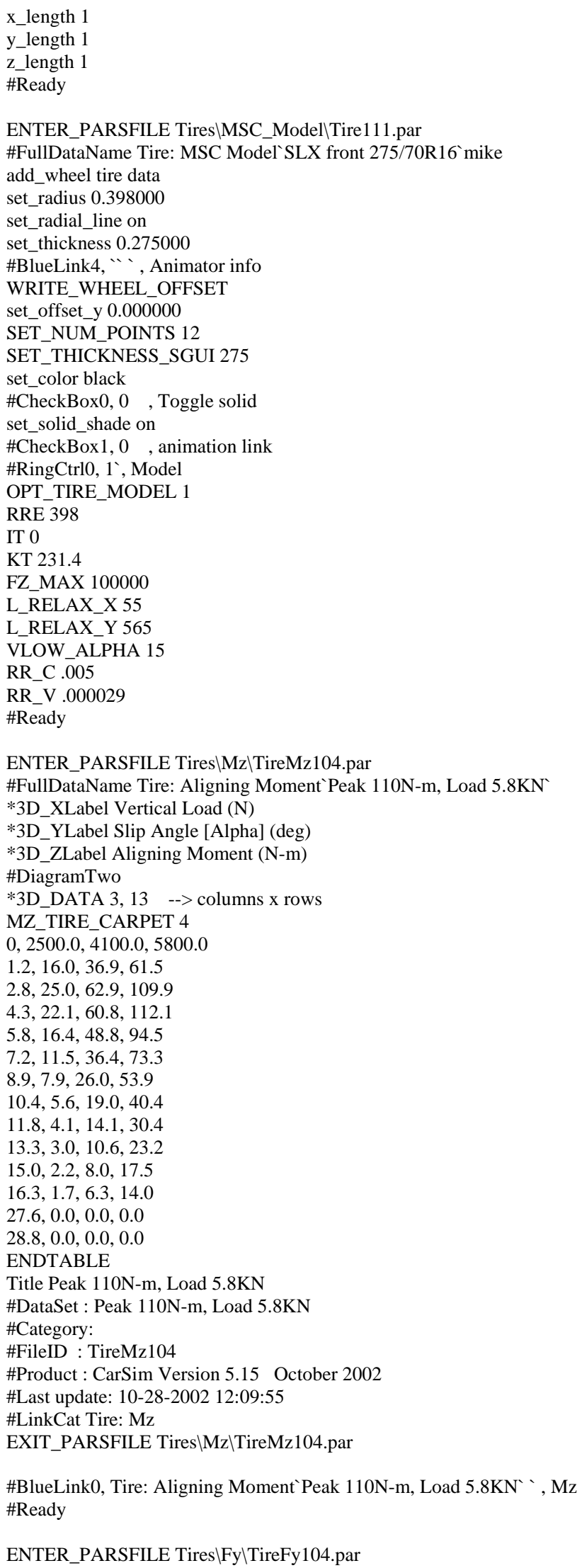

ENTER_PARSFILE Tires\Fy\TireFy104.par 
\#FullDataName Tire: Lateral Force `Slope 0.17, Load 6.7KN`

MU_REF_Y 0.87

*3D_XLabel Vertical Load (N)

*3D_YLabel Slip Angle [Alpha] (deg)

*3D_ZLabel Absolute Lateral Tire Force $(\mathrm{N})$

\#DiagramTwo

*3D_DATA 3, 12 --> columns $x$ rows

FY_TIRE_CARPET 4

$0,2900.0,4800.0,6700.0$

$1.2,993.3,1344.6,1579.6$

$2.9,2110.3,2966.7,3559.0$

4.4, 2648.6, 3850.3, 4717.3

6.0, 2888.7, 4296.6, 5346.2

7.6, 2979.9, 4497.3, 5654.9

9.2, 2998.2, 4570.0, 5788.8

$10.8,2979.1,4567.7,5812.2$

12.4, 2942.7, 4529.6, 5781.7

$14.0,2897.7,4472.1,5720.7$

15.6, 2844.2, 4398.3, 5635.3

$17.0,2796.6,4329.8,5553.1$

20.0, 2796.6, 4329.8, 5553.1

ENDTABLE

Title Slope 0.17, Load 6.7KN

\#DataSet : Slope 0.17, Load 6.7KN

\#Category:

\#FileID : TireFy104

\#Product : CarSim Version 5.15 October 2002

\#Last update: 10-28-2002 12:09:56

\#LinkCat Tire: Fy

EXIT_PARSFILE Tires\Fy\TireFy104.par

\#BlueLink1, Tire: Lateral Force`Slope 0.17, Load 6.7KN` `, Fy

\#Ready

ENTER_PARSFILE Tires\Fx\TireFx103.par

\#FullDataName Tire: Longitudinal Force 'Peak 20\% Slip, Load 6.7KN`

MU_REF_X 0.89

*3D_XLabel Vertical Load (N)

*3D_YLabel Abs. Slip Ratio [Kappa] (-)

*3D_ZLabel Absolute Longitudinal Tire Force (N)

\#DiagramTwo

*3D DATA 3, 12 --> columns $x$ rows

FX_TIRE_CARPET 4

$0,2900.0,4800.0,6700.0$

$0.009,335.625,559.562,785.36$

$0.013,530.107,883.918,1240.425$

$0.027,1094.347,1815.034,2527.206$

$0.067,2326.209,3736.741,4998.142$

$0.133,2930.522,4581.301,5944.776$

$0.2,2998.326,4648.622,5979.575$

$0.267,2961.147,4576.08,5866.472$

$0.333,2900.795,4475.8,5728.702$

$0.4,2836.935,4373.485,5592.818$

$0.467,2775.123,4275.93,5465.168$

$0.533,2716.933,4184.816,5346.879$

$0.6,2662.641,4100.222,5237.549$

ENDTABLE

Title Peak 20\% Slip, Load 6.7KN

\#DataSet : Peak 20\% Slip, Load 6.7KN

\#Category:

\#FileID : TireFx103

\#Product : CarSim Version 5.15 October 2002

\#Last update: 10-28-2002 12:09:57

\#LinkCat Tire: Fx

EXIT_PARSFILE Tires\Fx\TireFx103.par

\#BlueLink2, Tire: Longitudinal Force`Peak 20\% Slip, Load 6.7KN` `, Fx \#Ready 
ENTER_PARSFILE Tires\Camber_Thrust|CamStiff101.par

\#FullDataName Tire: Camber Thrust Coefficient`Constant @ -60

\#DiagramOne0

KGAMMA_TABLE 2

$0.000000,-60.000000$

5000.000000, -60.000000

ENDTABLE

Title Constant @ -60

\#DataSet : Constant @-60

\#Category:

\#FileID : CamStiff101

\#Product : CarSim Version 5.15 October 2002

\#Last update: 10-28-2002 12:09:55

\#LinkCat Camber thrust

EXIT_PARSFILE Tires\Camber_Thrust|CamStiff101.par

\#BlueLink3, Tire: Camber Thrust Coefficient`Constant @ -60` , Camber thrust

\#BlueLink5, '”, External

Title SLX front 275/70R16 <mike>

\#DataSet : SLX front 275/70R16

\#Category: mike

\#FileID : Tire111

\#Product : CarSim Version 5.15 October 2002

\#Last update: 10-27-2003 15:13:04

\#LinkCat MSC model

EXIT_PARSFILE Tires $\backslash$ MSC_Model\Tire111.par

\#BlueLink10, Tire: MSC Model `SLX front 275/70R16` mike`, Lead unit right front tire \#MiscYellow1

\#ENDMYellow

iaxle 2

iside 1

add_reference_frame L2 Wheel

set_euler_angles yaw_roll_pitch

set_X_name $X \_L 2$

set_y_name Y_L2

set_Z_name Z_L2

set_yaw_name Yaw_L2

set_pitch_name Rot_L2

set_roll_name Roll_L2

set_scale_var_pitch 360

x_ref_length 1

y_ref_length 1

z_ref_length 1

x_length 1

y_length 1

z_length 1

\#Ready

ENTER_PARSFILE Tires\MSC_Model|Tire112.par

\#FullDataName Tire: MSC Model`SLX rear 275/70R16`mike

add_wheel tire data

set_radius 0.398000

set_radial_line on

set_thickness 0.275000

\#BlueLink4, “', Animator info

WRITE_WHEEL_OFFSET

set_offset_y 0.000000

SET_NUM_POINTS 12

SET_THICKNESS_SGUI 275

set_color black

\#CheckBox0, 0 , Toggle solid

set_solid_shade on

\#CheckBox1, 0 , animation link

\#RingCtrl0, 1`, Model

OPT_TIRE_MODEL 1

RRE 398

IT 0

KT 248.6 
FZ_MAX 100000

L_RELAX_X 55

L_RELAX_Y 565

VLOW_ALPHA 15

RR_C .005

RR_V .000029

\#Ready

ENTER_PARSFILE Tires\Mz\TireMz104.par

\#FullDataName Tire: Aligning Moment`Peak 110N-m, Load 5.8KN`

*3D_XLabel Vertical Load (N)

*3D_YLabel Slip Angle [Alpha] (deg)

*3D_ZLabel Aligning Moment (N-m)

\#DiagramTwo

*3D_DATA 3, 13 --> columns x rows

MZ TIRE CARPET 4

$0,2500.0,4100.0,5800.0$

$1.2,16.0,36.9,61.5$

$2.8,25.0,62.9,109.9$

$4.3,22.1,60.8,112.1$

$5.8,16.4,48.8,94.5$

$7.2,11.5,36.4,73.3$

8.9, 7.9, 26.0, 53.9

$10.4,5.6,19.0,40.4$

$11.8,4.1,14.1,30.4$

13.3, 3.0, 10.6, 23.2

$15.0,2.2,8.0,17.5$

$16.3,1.7,6.3,14.0$

27.6, $0.0,0.0,0.0$

28.8, $0.0,0.0,0.0$

ENDTABLE

Title Peak 110N-m, Load 5.8KN

\#DataSet : Peak 110N-m, Load 5.8KN

\#Category:

\#FileID : TireMz104

\#Product : CarSim Version 5.15 October 2002

\#Last update: 10-28-2002 12:09:55

\#LinkCat Tire: $\mathrm{Mz}$

EXIT_PARSFILE Tires\Mz\TireMz104.par

\#BlueLink0, Tire: Aligning Moment`Peak 110N-m, Load 5.8KN`` , Mz \#Ready

ENTER_PARSFILE Tires\Fy\TireFy104.par

\#FullDataName Tire: Lateral Force 'Slope 0.17, Load 6.7KN`

MU_REF_Y 0.87

*3D_XLabel Vertical Load (N)

*3D_YLabel Slip Angle [Alpha] (deg)

*3D_ZLabel Absolute Lateral Tire Force (N)

\#DiagramTwo

*3D_DATA 3, 12 --> columns x rows

FY TIRE_CARPET 4

0, 2900.0, 4800.0, 6700.0

$1.2,993.3,1344.6,1579.6$

$2.9,2110.3,2966.7,3559.0$

$4.4,2648.6,3850.3,4717.3$

6.0, 2888.7, 4296.6, 5346.2

7.6, 2979.9, 4497.3, 5654.9

9.2, 2998.2, 4570.0, 5788.8

$10.8,2979.1,4567.7,5812.2$

12.4, 2942.7, 4529.6, 5781.7

14.0, 2897.7, 4472.1, 5720.7

15.6, 2844.2, 4398.3, 5635.3

17.0, 2796.6, 4329.8, 5553.1

20.0, 2796.6, 4329.8, 5553.1

ENDTABLE

Title Slope 0.17, Load 6.7KN

\#DataSet : Slope 0.17, Load 6.7KN

\#Category: 
\#FileID : TireFy104

\#Product : CarSim Version 5.15 October 2002

\#Last update: 10-28-2002 12:09:56

\#LinkCat Tire: Fy

EXIT_PARSFILE Tires\Fy\TireFy104.par

\#BlueLink1, Tire: Lateral Force`Slope 0.17, Load 6.7KN` `, Fy

\#Ready

ENTER_PARSFILE Tires\Fx\TireFx103.par

\#FullDataName Tire: Longitudinal Force Peak 20\% Slip, Load 6.7KN`

MU_REF X 0.89

*3D_XLabel Vertical Load (N)

*3D_YLabel Abs. Slip Ratio [Kappa] (-)

*3D_ZLabel Absolute Longitudinal Tire Force (N)

\#DiagramTwo

*3D_DATA 3, 12 --> columns x rows

FX_TIRE_CARPET 4

$0,2900.0,4800.0,6700.0$

$0.009,335.625,559.562,785.36$

$0.013,530.107,883.918,1240.425$

$0.027,1094.347,1815.034,2527.206$

$0.067,2326.209,3736.741,4998.142$

$0.133,2930.522,4581.301,5944.776$

0.2 , 2998.326, 4648.622, 5979.575

$0.267,2961.147,4576.08,5866.472$

$0.333,2900.795,4475.8,5728.702$

$0.4,2836.935,4373.485,5592.818$

$0.467,2775.123,4275.93,5465.168$

$0.533,2716.933,4184.816,5346.879$

$0.6,2662.641,4100.222,5237.549$

ENDTABLE

Title Peak 20\% Slip, Load 6.7KN

\#DataSet : Peak 20\% Slip, Load 6.7KN

\#Category:

\#FileID : TireFx103

\#Product : CarSim Version 5.15 October 2002

\#Last update: 10-28-2002 12:09:57

\#LinkCat Tire: Fx

EXIT_PARSFILE Tires $\backslash F x \backslash T i r e F x 103 . p a r$

\#BlueLink2, Tire: Longitudinal Force`Peak 20\% Slip, Load 6.7KN` `, Fx \#Ready

ENTER_PARSFILE Tires\Camber_Thrust|CamStiff101.par

\#FullDataName Tire: Camber Thrust Coefficient`Constant @ -60

\#DiagramOne0

KGAMMA_TABLE 2

$0.000000,-60.000000$

$5000.000000,-60.000000$

ENDTABLE

Title Constant @-60

\#DataSet : Constant @ -60

\#Category:

\#FileID : CamStiff101

\#Product : CarSim Version 5.15 October 2002

\#Last update: 10-28-2002 12:09:55

\#LinkCat Camber thrust

EXIT_PARSFILE Tires\Camber_Thrust|CamStiff101.par

\#BlueLink3, Tire: Camber Thrust Coefficient`Constant @ -60` , Camber thrust \#BlueLink5, “' , External

Title SLX rear 275/70R16 <mike>

\#DataSet: SLX rear 275/70R16

\#Category: mike

\#FileID : Tire112

\#Product : CarSim Version 5.15 October 2002

\#Last update: 10-27-2003 15:13:05

\#LinkCat MSC model 


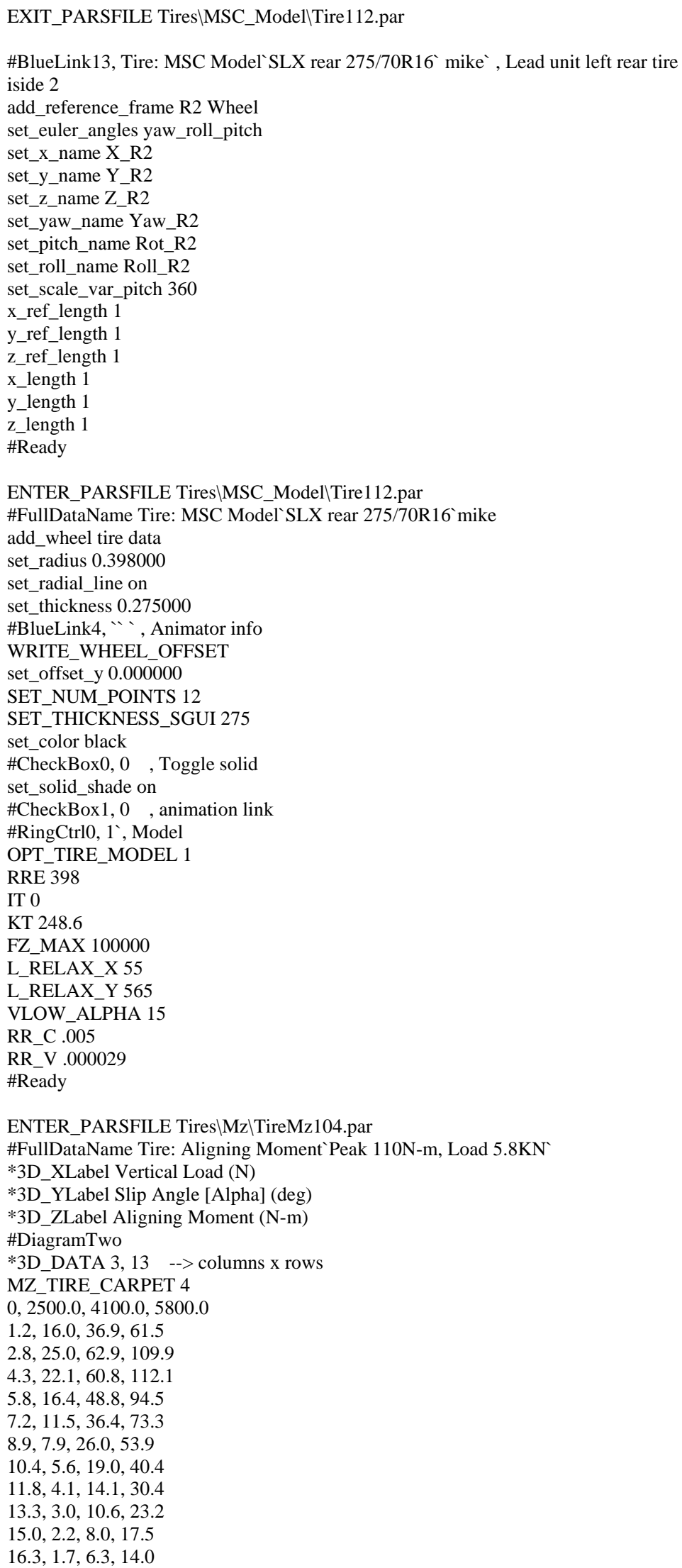




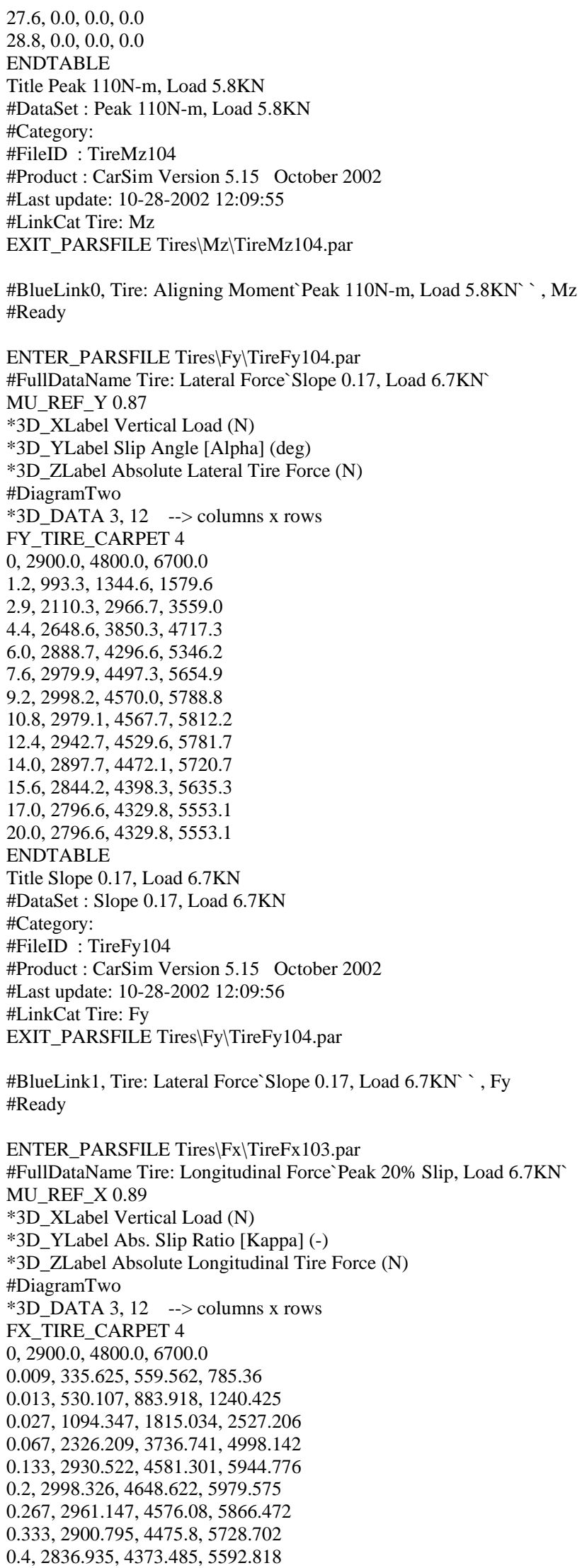


$0.467,2775.123,4275.93,5465.168$

$0.533,2716.933,4184.816,5346.879$

$0.6,2662.641,4100.222,5237.549$

ENDTABLE

Title Peak 20\% Slip, Load 6.7KN

\#DataSet : Peak 20\% Slip, Load 6.7KN

\#Category:

\#FileID : TireFx103

\#Product : CarSim Version 5.15 October 2002

\#Last update: 10-28-2002 12:09:57

\#LinkCat Tire: Fx

EXIT_PARSFILE Tires $\backslash F x \backslash T i r e F x 103 . p a r$

\#BlueLink2, Tire: Longitudinal Force`Peak 20\% Slip, Load 6.7KN` `, Fx

\#Ready

ENTER_PARSFILE Tires\Camber_Thrust|CamStiff101.par

\#FullDataName Tire: Camber Thrust Coefficient`Constant @ -60

\#DiagramOne0

KGAMMA TABLE 2

$0.000000,-60.000000$

$5000.000000,-60.000000$

ENDTABLE

Title Constant @ -60

\#DataSet : Constant @ -60

\#Category:

\#FileID : CamStiff101

\#Product: CarSim Version 5.15 October 2002

\#Last update: 10-28-2002 12:09:55

\#LinkCat Camber thrust

EXIT_PARSFILE Tires\Camber_Thrust|CamStiff101.par

\#BlueLink3, Tire: Camber Thrust Coefficient`Constant @ -60` ’ Camber thrust

\#BlueLink5, “' , External

Title SLX rear 275/70R16 <mike>

\#DataSet : SLX rear 275/70R16

\#Category: mike

\#FileID : Tire112

\#Product : CarSim Version 5.15 October 2002

\#Last update: 10-27-2003 15:13:05

\#LinkCat MSC model

EXIT_PARSFILE Tires\MSC_Model $\backslash T i r e 112 . p a r$

\#BlueLink14, Tire: MSC Model `SLX rear 275/70R16` mike`, Lead unit right rear tire \#MiscYellow2

\#ENDMYellow

Title SLX assembly left $<$ mike $>$

\#DataSet : SLX assembly left

\#Category: mike

\#FileID : Vehicle156

\#Product : CarSim Version 5.15 October 2002

\#Last update: 10-27-2003 15:17:00

\#LinkCat Ind_SA

EXIT_PARSFILE Vehicles\Assembly|Vehicle156.par

\#BlueLink2, Vehicle: Assembly`SLX assembly left`mike`, Vehicle

\#Ready

ENTER_PARSFILE ControlISteerlSteer101.par

\#FullDataName Control: Steering (Open Loop) `No steer

OPT_DRIVER_MODEL 0

\#DiagramOne0

STEER SW TABLE 2

$0.000000,0.000000$

$1.000000,0.000000$

ENDTABLE

Title No steer

\#DataSet : No steer

\#Category: 
\#FileID : Steer101

\#Product : CarSim Version 5.15 October 2002

\#Last update: 10-28-2002 12:11:26

\#LinkCat Open-loop steer control

EXIT_PARSFILE ControllSteerlSteer101.par

\#BlueLink3, Control: Steering (Open Loop) `No steer` ’, Input1

\#Ready

ENTER_PARSFILE Control/Braking\ConBrk100.par

\#FullDataName Control: Braking`No Braking`

\#DiagramOne0

PBK_CON_TABLE 2

$0.000000,0.000000$

$10.000000,0.000000$

ENDTABLE

Title No Braking

\#DataSet : No Braking

\#Category:

\#FileID : ConBrk100

\#Product : CarSim Version 5.15 October 2002

\#Last update: 10-28-2002 12:11:32

\#LinkCat Brake control

EXIT_PARSFILE Control|Braking\ConBrk100.par

\#BlueLink4, Control: Braking `No Braking ` , Input1

\#Ready

*Road Surface information deleted..

*3D_DATA 2, 2 --> columns x rows

MU_ROAD_CARPET 3

$0,0.0,1.0$

$0.0,1.1,1.1$

$1.0,1.1,1.1$

ENDTABLE

Title $1.1<$ mike $>$

\#DataSet : 1.1

\#Category: mike

\#FileID : RdFric113

\#Product: CarSim Version 5.15 October 2002

\#Last update: 10-27-2003 15:13:08

\#LinkCat Road: friction

EXIT_PARSFILE Roads\Friction\RdFric113.par

ENTER_PARSFILE Models\SimulinklCmex108.par

\#FullDataName Models: Simulink`J-turn simulink mdl`mike

\#RunMdlFile C:ICarSim5\Programs\Simulink_MDL \J_turn_switch.mdl

\#AltPath c:ICarSim5\Programs\Simulink_MDL

\#RunSimCat Ind Ind 'C: ICarSim5\PROGRAMS।Solvers\S functionli_i_mex.dll

\#RunSimCat Ind_SA C:ICarSim5।PROGRAMSISolvers\S_functionli_s_mex.dll

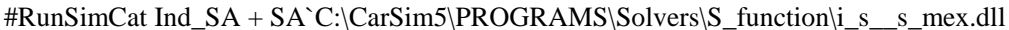

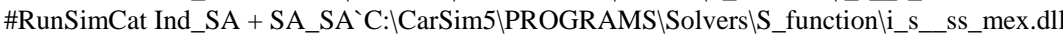

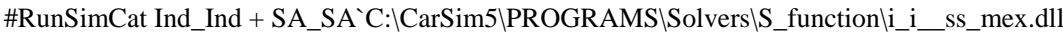

\#RunSimCat Ind_Ind + SA C C:ICarSim5\PROGRAMS ISolvers\S_functionli_i_s_mex.dll

*Sim_Code1 Ind_Ind

*Sim_Code2 Ind SA

*Sim_Code3 Ind_SA + SA

*Sim_Code4 Ind SA + SA SA

*Sim_Code5 Ind_Ind + SA_SA

*Sim_Code6 Ind Ind + SA

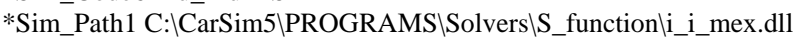

*Sim_Path2 C:ICarSim5\PROGRAMS SolversIS functionli_s mex.dll

*Sim_Path3 C:ICarSim5\PROGRAMS ISolvers\S_functionli_s_s_mex.dll

*Sim_Path4 C:ICarSim5\PROGRAMS।SolversIS_functionli_s_ss_mex.dl

*Sim_Path5 C:ICarSim5।PROGRAMSISolversIS_functionli_i_ss_mex.dll

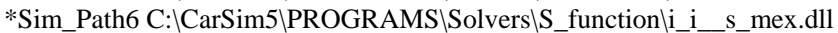

*Sim_Path7 C:|CarSim5

*Sim_Path8 C:\CarSim5 
*Sim_Path9 C:\CarSim5।

*Sim_Path10 C:\CarSim5।

*Sim_Path11 C:\CarSim5

*Sim_Path12 C:|CarSim5

*Sim_Path13 C:ICarSim5

*Sim Path14 C:|CarSim5

*Sim_Path15 C:|CarSim5

*Sim_Path16 C:|CarSim5

*Sim_Path17 C:|CarSim5

*Sim_Path18 C:|CarSim5

*Sim_Path19 C:|CarSim5

*Sim Path20 C:ICarSim5

*MDL_PATH C:ICarSim5\Programs\Simulink_MDL JJ_turn_switch.mdl

*ALTERNATE_PATH c:ICarSim5\Programs/Simulink_MDL

\#RingCtrl0, 6', Ring

N_CODES 6

\#MiscYellow0

\#ENDMYellow

\#Ready

ENTER_PARSFILE Generic \Lists\GList127.par

\#FullDataName Generic Data Lists`simulink Import/Export `mike

\#MiscYellow0

OPT_ALL_IMPORT 0

OPT_ECHO_IMPORT 1

begin_set_import

IMP_Steer_SW

end_set_import

\#ENDMYellow

\#MiscYellow1

OPT_ALL_EXPORT 0

OPT_ECHO_EXPORT 1

begin_set_export

EXP_Ay

EXP_Roll

EXP_Steer_SW

EXP_Vx

EXP_Yo

EXP Xo

EXP_Z_L1

EXP_Z_L2

EXP_Z_R1

EXP_Z_R2

EXP_AVz

EXP_AVX

end_set_export

\#ENDMYellow

\#MiscYellow2

\#ENDMYellow

Title simulink Import/Export <mike >

\#DataSet : simulink Import/Export

\#Category: mike

\#FileID : GList127

\#Product : CarSim Version 5.15 October 2002

\#Last update: 10-27-2003 15:13:11

\#LinkCat Generic Lists

EXIT_PARSFILE Generic \Lists\GList127.par

\#BlueLink0, Generic Data Lists`simulink Import/Export `mike`, Link 1 \#Ready

ENTER_PARSFILE Generic \Lists\GList127.par

\#FullDataName Generic Data Lists`simulink Import/Export `mike

\#MiscYellow0

OPT_ALL_IMPORT 0

OPT_ECHO_IMPORT 1

begin_set_import

IMP_Steer_SW

end_set_import 


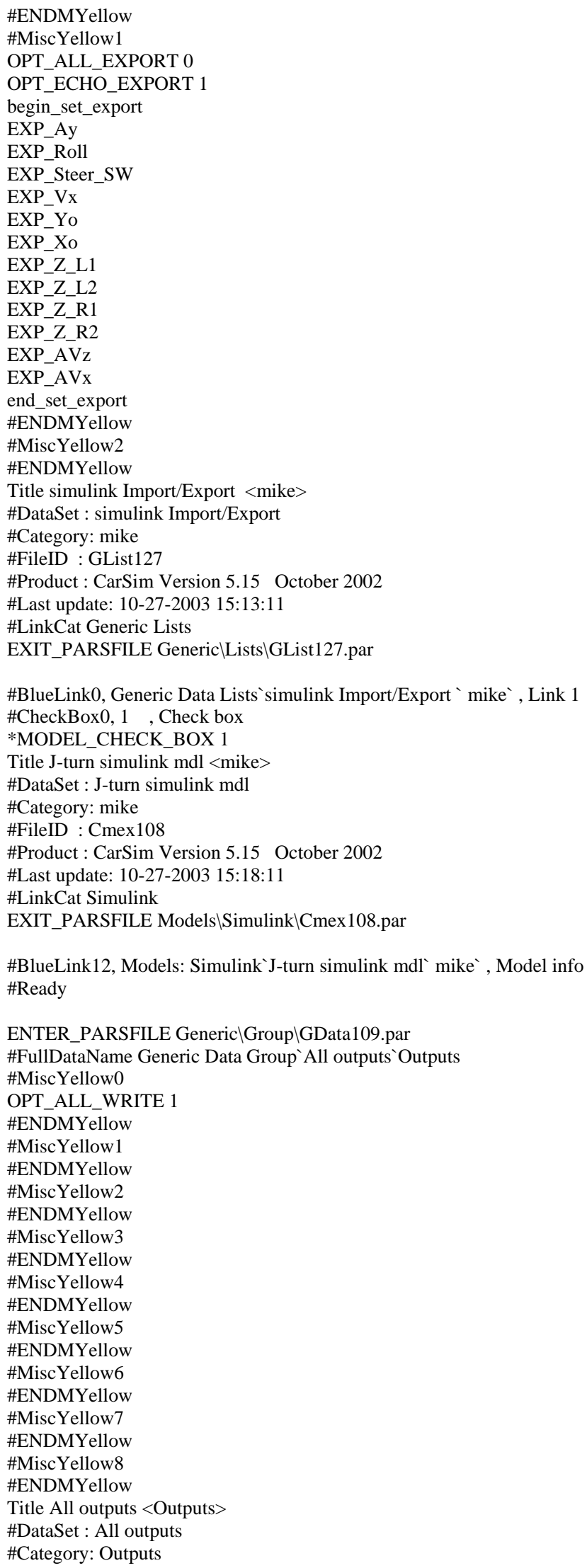


\#FileID : GData109

\#Product : CarSim Version 5.15 October 2002

\#Last update: 10-28-2002 12:11:23

\#LinkCat Generic group

EXIT_PARSFILE Generic\Group\GData109.par

\#BlueLink13, Generic Data Group`All outputs` Outputs`, Output Variables

Opt_SC 2

\#Ready

ENTER_PARSFILE ControllSpeed_tISpdTime102.par

\#FullDataName Control: Speed vs. Time`drop throttle $40 \mathrm{mph}$ `mike

SPEED_KI 0.157

SPEED_KP 0.141

OPT_VMIN -1

\#DiagramOne0

SPEED_TIME_TABLE 2

$0.000000,64.370000$

$5.000000,64.370000$

$5.010000,0.000000$

20.000000, 0.000000

ENDTABLE

Title drop throttle $40 \mathrm{mph}<$ mike $>$

\#DataSet : drop throttle $40 \mathrm{mph}$

\#Category: mike

\#FileID : SpdTime102

\#Product : CarSim Version 5.15 October 2002

\#Last update: 10-27-2003 15:13:12

\#LinkCat Speed vs. time

EXIT_PARSFILE ControliSpeed_tISpdTime102.par

\#BlueLink7, Control: Speed vs. Time`drop throttle 40 mph `mike`, Speed link \#BlueLink8, Control: Speed vs. Station“ ”, Speed link

Speed 120

TSTOP 20

TSTART -3

Sstart -50

sgui_Sstop 1000

TSTEP 0.001

*TSTEP_OUT 0.01

*FREQ_MODEL 1000

*FREQ_OUT 100

TSTOP_WRITE 20

OPT_WRITE_BASIS 1

iprint 10

Sstop 1000

\#RingCtrl0, 2', Speed Control

\#RingCtrl1, 0', Placeholder

\#RingCtrl2, 1', Stop Station

OPT_SSTOP 1

\#RingCtrl3, 0`, Direction of vehicle on road

\#CheckBox0, 1 , Multiple Plot

\#CheckBox1, 0 , Overlay Plots

\#CheckBox2, 1 , Show More Settings

\#CheckBox3, 0 , Run RT-Animation

\#MiscYellow0

\#ENDMYellow

\#BlueLink15, Plot: Setup `Vx -- longitudinal speed`Vehicle Motion` , Plot \#1

\#BlueLink16, Plot: Setup`Ay -- lat. accel. of CG's` Vehicle Motion`, Plot \#2

\#BlueLink17, Plot: Setup 'Roll -- sprung masses` Vehicle Motion`, Plot \#3

\#BlueLink18, Plot: Setup `Y vs X -- trajectory` Tracking`, Plot \#4

\#BlueLink19, Plot: Setup`Steer SW -- steering wheel`Steering`, Plot \#5

\#BlueLink20, Plot: Setup “ ' , Plot \#6

\#BlueLink21, Plot: Setup “ ', Plot \#7

\#BlueLink22, Plot: Setup “ ', Plot \#8

\#BlueLink23, CarSim Run Control” “, Overlay \#1

\#BlueLink24, “' , Overlay \#2

\#BlueLink25, “' , Overlay \#3

Title SLX home2 pars output $<$ mike $>$ 
\#DataSet : SLX home2 pars output

\#Category: mike

\#FileID : Run377

\#Product : CarSim Version 5.15 October 2002

\#Last update: 10-27-2003 15:23:17

\#LinkCat Run

EXIT_PARSFILE Runs\Run377.par

END 